PNL-10060

UC-603

\title{
Identification of Radionuclides of Concern in Hanford Site Environmental Cleanup
}

\author{
R. W. Perkins \\ U. P. Jenquin
}

August 1994

Prepared for

the U.S. Department of Energy

under Contract DE-AC06-76RLO 1830

Pacific Northwest Laboratory

Richland, Washington 99352

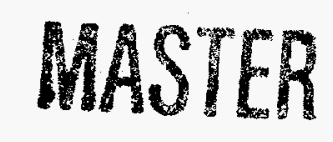

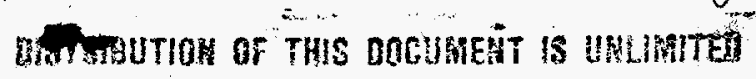




\section{DISCLAIMER}

This report was prepared as an account of work sponsored by an agency of the United States Government. Neither the United States Government nor any agency thereof, nor any of their employees, make any warranty, express or implied, or assumes any legal liability or responsibility for the accuracy, completeness, or usefulness of any information, apparatus, product, or process disclosed, or represents that its use would not infringe privately owned rights. Reference herein to any specific commercial product, process, or service by trade name, trademark, manufacturer, or otherwise does not necessarily constitute or imply its endorsement, recommendation, or favoring by the United States Government or any agency thereof. The views and opinions of authors expressed herein do not necessarily state or reflect those of the United States Government or any agency thereof. 


\section{DISCLAIMER}

Portions of this document may be illegible in electronic image products. Images are produced from the best available original document. 


\section{Executive Summary}

The purpose of this document is to consider which radionuclides should be included in conducting environmental surveys relative to site remediation at Hanford. During the operation of the Hanford site, the fission product radionuclides and a large number of activation products including the transuranic radionuclides were formed. The reactor operations and subsequent chemical processing and metallurgical operations resulted in the environmental release of gaseous and liquid effluents containing some radionuclides; however, the majority of the radionuclides were stored in waste tanks or disposed to trenches and cribs. Since some contamination of both soils and subsurface waters occurred, one must decide which radionuclides may remain in sufficient amounts to be of concern at the time when site remediation is to be complete.

Many of the radionuclides which have constituted the principal hazard during site operation have half-lives on the order of a year or less; therefore, they will have decayed to insignificant amounts by the year 2030, a possible date for completion of the remediation process. An example of how decay will take care of decontamination for radionuclides with half-lives of a year or less is illustrated below for a hypothetical radionuclide with a one-year half-life.

$\begin{array}{cl}\text { Decay Period (years) } & \text { Fraction of Original Activity Remaining } \\ 10 & \text { One-thousandth } \\ 20 & \text { One-millionth } \\ 30 & \text { One-billionth } \\ 40 & \text { One-trillionth }\end{array}$

Decay to one-trillionth of the original activity would reduce one curie of a radionuclide [2.22 $\times 10^{12}$ disintegrations per minute $\left.(\mathrm{dpm})\right]$ to one picocurie $(2.22 \mathrm{dpm})$. If the half-life were two years, then the decay period of 40 years would decrease the content to one-millionth, and 80 years would be required to reduce it to one-trillionth of its original activity.

Since contamination of the environs was usually in quantities ranging from far less than one curie to perhaps thousands of curies, it should be clear that for radionuclides with half-lives of less than a year (unless they are supported by long-lived parents) natural depletion by radioactive decay may have already reduced their concentration to negligible quantities, or it certainly will have by the year 2030 .

To estimate the "relative hazard" or concern for those radionuclides which may be present in significant quantities after decay periods of tens, hundreds and thousands of years, their concentrations which would be present in irradiated fuel after various decay periods were calculated by use of the ORIGEN2 Code. ${ }^{(1,2)}$ These remaining activities were then divided by their "acceptable concentrations" (Derived Concentration Guide values) ${ }^{(3,4)}$ in drinking water and also in air (as a guide 
for inhalation of resuspended soil) to provide an indication of their "relative hazard" after specific decay periods. In addition, calculations were made of the relative hazard from external radiation that would result if these radionuclides were deposited on the surface of Hanford soils. These "relative hazards" (which would only be relative if no fractionation of their initial abundances other than radioactive decay had occurred) provide a reasonable basis for selecting radionuclides which should be included in pre-remediation and subsequent surveys.

Based on this procedure and factoring in other considerations, including special isotope production processes and possible food chain concentration processes, it is concluded that the following radionuclides should probably be included in preremediation site surveys. Most of these are anthropogenic radionuclides of long-term concern. However, certain natural radionuclides are included since they provide a basis for estimating the primary source of these radionuclides which could be natural or man-made. The radionuclides include:

$\begin{array}{lll}\text { H-3 } & \text { Eu-152 } & \text { Pu-238 } \\ \text { K-40 } & \text { Eu-154 } & \text { U-238 } \\ \text { Co-60 } & \text { Eu-155 } & \text { Pu-239 } \\ \text { Sr-90 } & \text { Ra-226 } & \text { Pu-240 } \\ \text { Tc-99 } & \text { Th-232 } & \text { Am-241 } \\ \text { Ru-106 } & \text { U-232 } & \text { Pu-241 } \\ \text { Sb-125 } & \text { U-233 } & \text { Cm-244 } \\ \text { I-129 } & \text { U-234 } & \\ \text { Cs-134 } & \text { U-235 } & \\ \text { Cs-137 } & \text { Np-237 } & \end{array}$




\section{Contents}

Executive Summary $\ldots \ldots \ldots \ldots \ldots \ldots \ldots \ldots \ldots \ldots \ldots \ldots \ldots$ iii

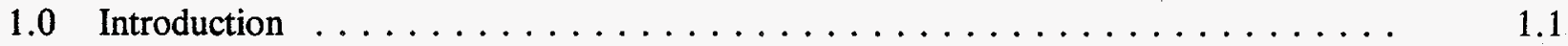

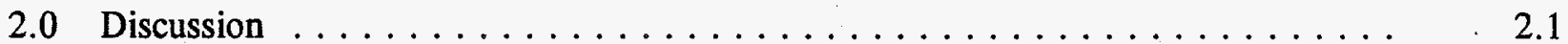

3.0 Contributions From Fallout Radionuclides $\ldots \ldots \ldots \ldots \ldots \ldots \ldots \ldots \ldots$

4.0 Recommendations $\ldots \ldots \ldots \ldots \ldots \ldots \ldots \ldots \ldots \ldots \ldots \ldots \ldots \ldots \ldots$

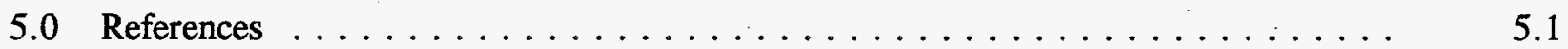

Appendix A - Basis for ORIGEN2 Calculation $\ldots \ldots \ldots \ldots \ldots \ldots \ldots \ldots \ldots \ldots$ A.1

Appendix B - Selection of Derived Concentration Guide (DCG) Values $\ldots \ldots \ldots \ldots$. . . .

Appendix C - External Dose-Rate Conversion Factors $\ldots \ldots \ldots \ldots \ldots \ldots$ C. 1 


\section{Tables}

2.1 Radionuclide Content of Fission Products in Hanford Reactor Fuel at

Various Decay Times $\ldots \ldots \ldots \ldots \ldots \ldots \ldots \ldots$

2.2 Radionuclide Content of Activation Products in Hanford Reactor Fuel and Cladding at Various Decay Times $\ldots \ldots \ldots \ldots \ldots \ldots$

2.3 Radionuclide Content of Actinides in Hanford Reactor Fuel for

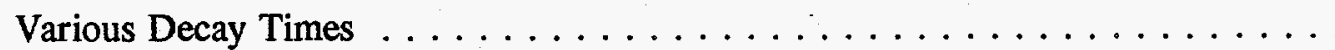

2.4 Relative Inhalation Hazard of Fission Products in Hanford Reactor Fuel at Various Decay Times $\ldots \ldots \ldots \ldots \ldots \ldots \ldots$

2.5 Relative Inhalation Hazard of Activation Products in Hanford Reactor Fuel and Cladding at Various Decay Times $\ldots \ldots \ldots \ldots \ldots \ldots$

2.6 Relative Inhalation Hazard of Activation in Hanford Reactor Fuel for Various Decay Times $\ldots \ldots \ldots \ldots \ldots \ldots \ldots \ldots$

2.7 Relative Ingestion Hazard of Fission Products in Hanford Reactor Fuel

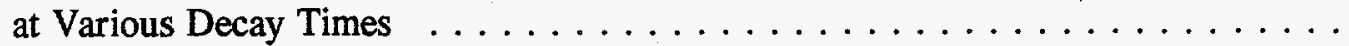

2.8 Relative Ingestion Hazard of Activation Products in Hanford Reactor Fuel and Cladding at Various Decay Times $\ldots \ldots \ldots \ldots \ldots \ldots$

2.9 Relative Ingestion Hazard of Actinides in Hanford Reactor Fuel for Various Decay Times $\ldots \ldots \ldots \ldots \ldots \ldots \ldots \ldots \ldots \ldots \ldots \ldots \ldots \ldots \ldots$

2.10 Relative Radiation Hazard of Surface-Deposited Fission Products from Hanford Reactor Fuel for Various Decay Times $\ldots \ldots \ldots \ldots \ldots \ldots$

2.11 Relative Radiation Hazard of Surface-Deposited Activation Products from Hanford Reactor Fuel and Cladding at Various Decay Times . . . . . . . . . .

2.12 Relative Radiation Hazard of Surface-Deposited Actinides from Hanford Reactor Fuel for Various Decay Times . . . . . . . . . . . . .

3.1 Radionuclide Content of Fission Products from a $10 \mathrm{KT}$ Burst at Various Decay Times . . . . . . . . . . . . . . . . . . .

3.2 Radionuclide Content of Actinides from a $10 \mathrm{KT}$ Burst at Various Decay Times . . $\quad 3.4$

4.1 Suggested Radionuclides to be Included in Surveys Relative to Site Remediation . . $\quad 4.2$ 


\subsection{Introduction}

The purpose of this work is to provide an estimate of the relative importance of the various radionuclides that were produced during the operation of the Hanford project. Specifically, it is designed to identify those radionuclides which may be present in significant amounts after a decay period comparable to that expected for the site cleanup operation. This information, together with Derived Concentration Guide (DCG) values ${ }^{(3,4)}$ and external radiation Dose Conversion Factors $(\mathrm{DCF})^{(5)}$ for each radionuclide, could then provide a basis for selecting the spectrum of radionuclides which should be included in preremediation and subsequent site surveys.

During the production period of the Hanford Site, eight plutonium production reactors, together with the N-reactor which produced both plutonium and electric power, operated and produced fission and activation by-products. Irradiated fuels were processed for recovery of Pu-239 which was the principal product of the operation. In addition, two test reactors, the Plutonium Recycle Test Reactor (PRTR) and the Fast Flux Test Facility (FFTF) were operated and their fuels were reprocessed for material recovery. While literally thousands of fission and activation products were produced, most of these have relatively short half-lives and have decayed to insignificant concentrations since the reprocessing of fuels for plutonium recovery or other product recovery has ceased.

The relative concentrations of radionuclides which would still be present for periods out through 3000 years from now, but with particular emphasis on a period of about 40 years from the present, have been calculated with the ORIGEN $2^{(1,2)}$ computer code. In carrying out this work, certain assumptions were made as to the irradiation time, the neutron energy spectrum, and the total megawatt days per ton to which this fuel was exposed. This provided a basis for calculating the actual and the relative amounts of the radionuclides that were present in fuel at the end of the irradiation process and for the various decay times of interest following irradiation. The ORIGEN2 code was employed for this purpose. The calculations were made using a cross-section library developed for the $\mathrm{N}$ reactor fuel/moderator design. However, analyses indicate that ORIGEN2 would provide a reasonable estimation of the radionuclide spectrum that was produced in the eight Hanford production reactors. ${ }^{(6)}$ By using results of the ORIGEN2 calculation, together with the $\mathrm{DCG}^{(3,4)}$ and external DCF values ${ }^{(5)}$ for each of the radionuclides, it was possible to determine the "relative hazards" or concern of each radionuclide as a function of time after production in the nuclear reactors. 


\subsection{Discussion}

In the past, the main effort at the Hanford Site was the production of weapons-grade plutonium. This was accomplished by irradiation of natural and slightly enriched uranium in the eight production reactors and in the $\mathrm{N}$ dual-purpose-reactor. The chemical separations process that followed fuel irradiation separated the plutonium from the fission, activation, and transuranic radionuclides, as well as the remaining uranium target material. Since plutonium recovery was the object of the process, its presence in the wastes and effluents was minimal, and therefore the amount of plutonium that might be a problem in the site remediation is far less than the amount produced.

In addition to the production of plutonium, some U-233 was produced. This radionuclide is a fissile isotope and was produced as a product for the thorium breeder reactor program. It was produced by irradiation of Th-232 target material. Irradiation of Th-232 produced Th-233 which decayed via $\mathrm{Pa}-233$ to $\mathrm{U}-233$. For this reason, $\mathrm{U}-233$ production, while not specifically included in the tables of "relative hazards," may be of somewhat greater concern than indicated. However, its long half-life of $1.59 \times 10^{5}$ years limits its specific activity and thus its concern from the site cleanup standpoint. U-232, a by-product in U-233 production, is also of concern because of its short half-life (70 years) and associated high specific activity and that of its short-lived progeny. Its production involves the reaction Th-232(n,2n)Th-231, decay of Th-231 to Pa-231 and a neutron capture to form Pa-232 which, in turn, decays to U-232. The relatively short half-life of U-232 results in a high specific activity of both itself and its short-lived daughters, and thus it is of concern. Several other radionuclides were produced in the Hanford reactors and were separated as by-products and employed for research, heat or power sources, irradiation sources, and other applications. These included Pu-238, Np-237, Cs-137, Sr-90, and Pm-147. However, the amounts that were separated were very small compared with their total production and, therefore, the amounts removed would not need to be considered when estimating the potential hazard associated with these radionuclides in the Hanford environs.

The ORIGEN2 code ${ }^{(1,2)}$ was employed to estimate the relative concern of each of the fission products, activation products, and transuranic radionuclides which were produced in the operation of the Hanford Site. As indicated in the Introduction, the cross-section library was actually designed for the Hanford $\mathrm{N}$ reactor, but provides a reasonably good estimate of the actual and relative production rates of radionuclides in the eight Hanford Plutonium production reactors. ${ }^{(6)}$ It was assumed that all the fuel from the eight production reactors was irradiated to the average exposure of $728 \mathrm{MWd} / \mathrm{MTU}$ (see Appendix A).

In carrying out this work, it was recognized that most of the fission products, activation products, and many of the transuranic isotopes would have decayed to insignificance within a few years after their production.

If one were to assume that the Hanford Site cleanup would be completed in the year 2030 , then it would be appropriate to determine what the relative concentrations of Hanford-produced radionuclides would be at that time. Since this is roughly 40 years past cessation of the last Hanford reactor operation, then one can readily illustrate that for radionuclides with half-lives of less than one year, they will have decayed to one-trillionth, or less of their original activities. The example is given of a hypothetical radionuclide with a half-life of one year. After a 10-year decay period, such a radionuclide will have decayed by approximately one-thousandth of its original activity. During each additional 10-year 
period, the remaining radionuclides will again decay by the same amount as indicated below; and therefore, at the end of 40 years, they will have decayed to one-trillionth of their original activity.

$\begin{array}{ccc}\text { Decay Period (years) } & \text { Residual Activity } & \text { Fraction of Original Activity Remaining } \\ 0 & 1 \mathrm{Ci} & \\ 10 & 1 \mathrm{mCi} & \text { One-thousandth } \\ 20 & 1 \mu \mathrm{Ci} & \text { One-millionth } \\ 30 & 1 \mathrm{nCi} & \text { One-billionth } \\ 40 & 1 \mathrm{pCi} & \text { One-trillionth }\end{array}$

Thus, if one curie $\left(2.22 \times 10^{12} \mathrm{dpm}\right)$ of a radionuclide with a half-life of one year were produced, then 40 years later it will have decayed to one picocurie $(2.22 \mathrm{dpm})$. Obviously, another 40 years of decay would have reduced it to an additional one-trillionth or to $10^{24}$ of its original activity. Similarly, a radionuclide with a half-life of two years would decay to one-millionth in 40 years and one-trillionth in 80 years of its original concentration.

Even for a radionuclide with a half-life of five years, such as Co-60, its initial concentration will have been reduced to one-thousandth after 50 years and to one-millionth after 100 years. Keeping these facts in mind, one can better assess the importance of each of the reactor-generated radionuclides in any contemplated waste cleanup program. The approach in this document is to provide a simple, straightforward indication of the relative importance of including each of the radionuclides that was produced at Hanford in preremediation and subsequent site surveys.

It is recognized, of course, that during the release and following the release of radionuclides to the environment, there may be substantial fractionation. Nevertheless, this approach still provides a good indication of what radionuclides should be of concern.

To provide a further basis for selection of the radionuclides of concern, the Derived Concentration Guide, (DCG) value for each of the radionuclides was taken into account (see Appendix B). In order to appropriately employ these guides for each of the specific radionuclides, the relative concentrations of each radionuclide after specific decay periods of interest were divided by their DCG values. This approach was applied for the exposure pathways of inhalation and ingestion. In addition to these pathways of exposure, the relative hazards from direct external radiation exposure ("shine") were calculated, ${ }^{(5)}$ assuming the radionuclides were deposited on soil surfaces. The basic data for these calculations are given in Appendix $\mathrm{C}$, and the results are included in the tables which are explained below. 
Tables 2.1, 1-A through 1-I, Tables 2.2, 2-A through 2-I, and Tables 2.3, 3-A through 3-I summarize the concentrations of fission products, activation products, and the actinide radionuclides, respectively, that would be present in irradiated fuel following decay periods of 1 year, 3 years, 10 years, 30 years, 40 years, 100 years, 300 years, 1000 years, and 3000 years. In each case, only the 70 most abundant radionuclides are considered.

In Tables 2.4, 4-A through 4-I, Tables 2.5, 5-A through 5-I, and Tables 2.6, 6-A through 6-I the relative hazard, or relative concern, of each of these radionuclides is indicated for the inhalation pathway. Similarly, the relative hazard of the ingestion pathway (based on drinking water) is shown in Tables 2.7, 7-A through 7-I, Tables 2.8, 8-A through 8-I, and Tables 2.9, 9-A through 9-I. These tables were developed by dividing the relative abundances of each of the radionuclides by their DCG values in air and water, respectively (see Appendix B and Table 18).

Tables 2.10, 10-A through 10-I, Tables 2.11, 11-A through 11-I, and Tables 2.12, 12-A through 12-I summarize in a similar manner the relative hazard or concern of these radionuclides for the external radiation exposure pathway. These relative hazards were calculated by multiplying the relative concentrations of the radionuclides which would be present after various decay periods by their relative radiation dose per unit of radioactivity (curies) assuming surface deposition. These values are summarized in Appendix C.

The relative hazards, or relative concerns, listed in these tables can serve as a useful guide in determining which radionuclides may be of concern at the present time, at the estimated time of return of the Hanford Site to normal or limited usage, and at times, through 3000 years from the present. Since these values represent only the relative hazard for three possible pathways of exposure, the values for one pathway may only be compared within that pathway, and not with the other two pathways. While this provides a relatively simple method of determining which radionuclides should be measured in a preremediation or subsequent survey, it does not address the problem of food chain transfer which may concentrate some of these radionuclides in foods and, therefore, provide a greater relative hazard than that estimated by employing the DCG values in conjunction with the relative amounts of remaining radionuclides after specific decay periods. Food chain concentration factors should be taken into account where they may produce a significant effect on the potential hazards of any specific radionuclides.

An additional concern which is not addressed in the above tables is the production of U-233 (and inadvertently $\mathrm{U}-232$ ) by irradiation of Th-232 and the production of tritium by irradiation of Li- 6 . Therefore, in identifying radio nuclides which should be of concern in site surveys, the radionuclides Th-232, U-232, U-233, and tritium should be included. Tritium is, of course, a ternary fission product, and is therefore already included with the fission products but far greater amounts could have been released during tritium production. 
Table 2.1. Radionuclide Content of Fission Products in Hanford Reactor Fuel at Various Decay Times

. Tablo 1-A. Docay Timo - I Yoar

\begin{tabular}{|c|c|c|c|}
\hline & latf-Lito & Curies/kgU & Percont \\
\hline $\begin{array}{l}\text { PR14 } \\
\text { CE14 }\end{array}$ & $\begin{array}{l}\text { 17.28 Min. } \\
\text { 284.3 Days }\end{array}$ & $\begin{array}{l}29.44 \\
29.44\end{array}$ & $\begin{array}{l}26.44 \\
26.44\end{array}$ \\
\hline NB & 5.15 Days & 11.69 & 10.50 \\
\hline PM147 & 623 Years & 7.33 & 6.58 \\
\hline $\mathrm{RH}$ & 29.9 Sec. & .92 & 5.31 \\
\hline 6 &.$\infty$ & .92 & 5.31 \\
\hline ZR & Days & .43 & 4.87 \\
\hline & & & 3.06 \\
\hline CS & 18 & 2.38 & 2.14 \\
\hline $\mathrm{BA}$ & n. & 2.25 & 2.02 \\
\hline & $y s$ & 2.04 & 1.83 \\
\hline & eara & 2.04 & 1.83 \\
\hline & lays & & 1.35 \\
\hline & ays & & 37 \\
\hline$n$ & & & 17 \\
\hline RH & 56. & 88 & 0.304 \\
\hline & & 5 & 0.292 \\
\hline & & & 31 \\
\hline & iss & & 83 \\
\hline & 32 & & 0.142 \\
\hline & & & 0.108 \\
\hline & is & & 06 \\
\hline & 's & & 0.070 \\
\hline & is & & 0.050 \\
\hline S & & & 0.043 \\
\hline 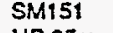 & gers & 0.044 & 0.040 \\
\hline$m$ & ays & 0.040 & 0.036 \\
\hline & & 0.0 & 0.013 \\
\hline & ears & 0.012 & 0.011 \\
\hline $9 m$ & Days & 0.0045 & $E-03$ \\
\hline & & 42 & $E-03$ \\
\hline & & 30 & $E-03$ \\
\hline & & & $2.6 E-03$ \\
\hline & 249 & 0.0 & $1.7 E-03$ \\
\hline & & & $E-04$ \\
\hline & ars & & $E-04$ \\
\hline$T$ & & & $E-04$ \\
\hline & & & $E-04$ \\
\hline 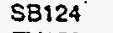 & & & $E-05$ \\
\hline 152 & ars & -05 & $E-05$ \\
\hline$T$ & & 55 & $E-05$ \\
\hline & & & $E-05$ \\
\hline A & c. & 5 & -05 \\
\hline & & & $1.5 E-05$ \\
\hline m & in. & 05 & $1.5 E-05$ \\
\hline & 64. & 5 & $=-0.6$ \\
\hline 515 & $M Y$ & -06 & $7.5 E-06$ \\
\hline & Days & 06 & $6.7 E-06$ \\
\hline M1 & ars & 06 & $4.5 E-05$ \\
\hline & 18 & 06 & $E-06$ \\
\hline 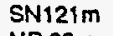 & ars & 06 & $=-06$ \\
\hline & ars & & $E-\infty$ \\
\hline 812 & ys & $3 E-\infty 6$ & 2.1E-O6 \\
\hline & & $4 E-\infty 6$ & $1.3 E-06$ \\
\hline & Days & $2 E-06$ & $1.1 E-06$ \\
\hline ton & e.7 & $2 E-06$ & $1.1 E-\infty 6$ \\
\hline 1129 & 15. & $8 E-07$ & $6.1 E-07$ \\
\hline DLu & 2.9 Years & $E-07$ & $4.6 \mathrm{E}-07$ \\
\hline 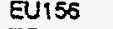 & & $E-07$ & $3.6 E-07$ \\
\hline TE123m & 119.7 Days & 2.0E-07 & $1.8 E-07$ \\
\hline & & & \\
\hline & & & -08 \\
\hline & & 2.1E-08 & $1.9 E-08$ \\
\hline & Days & & $E-09$ \\
\hline & 11.9 Days & $5.1 E-09$ & $4.6 \mathrm{E}-09$ \\
\hline & & $E-09$ & -09 \\
\hline IN114 & 1.198 Min. & $4.7 E-09$ & $4.3 E-09$ \\
\hline & 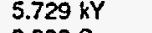 & $2.7 E-09$ & $2.4 E-09$ \\
\hline & 0.3 & 165 & $1.4 \mathrm{E}$ \\
\hline & & 09 & \\
\hline
\end{tabular}

Table 1-B. Decay Time $=3$ Yoare

\begin{tabular}{|c|c|c|c|}
\hline & Hatf-Lito & Curies/kgU & Cor \\
\hline & 284.3 Days & 4.958 & 10.0 \\
\hline & 17.28 Min. & 4.958 & \\
\hline$P M 147$ & 2.623 Years & 4.322 & \\
\hline $\operatorname{CS} 137$ & 30 Years & 2.271 & \\
\hline & $2.552 \mathrm{Min}$. & 2.149 & \\
\hline - & 7 Days & 1.945 & \\
\hline SR 90 & 2 Years & 1.944 & \\
\hline & 1.008 Years & 1.495 & \\
\hline RH1O6 & 29.9 Sec. & 1.495 & \\
\hline & ears & 0.226 & \\
\hline & 2.77 Years & 0.197 & \\
\hline 4 & gars & 0.104 & \\
\hline $\mathrm{im}$ & 7.2 Min. & 0.059 & \\
\hline & & 0.048 & \\
\hline & ers & 0.044 & \\
\hline & ars & 0.042 & 0. \\
\hline$\vec{E}$ & is & 0.012 & 0.0 \\
\hline & 12. & 0.010 & $0 . c$ \\
\hline 95 & 35. & 0.0 & 0.0 \\
\hline $\mathbf{Z}$ & ys & 0.0020 & $7.5 \mathrm{E}-$ \\
\hline & & 0.0 & 4 \\
\hline & ars & 0. & 4. \\
\hline $\mathbf{S}$ & 129 & 0.0 & 3. \\
\hline & & 34 & 3. \\
\hline Y9 & s & 0.0 & $2.3 E-C$ \\
\hline V119m & iys & 0.00054 & $2.0 E-03$ \\
\hline 15 & 2 & 0.0 & $1.3 \dot{E}-03$ \\
\hline Om & 249 & 0.0 & $9.4 E-0$ \\
\hline S & 50. & $6.6 \mathrm{E}-05$ & $2.5 \mathrm{E}-0$ \\
\hline EU152 & 13. & $5.5 E-05$ & $2.1 E-0$ \\
\hline $7=$ & 1.5 & $4.7 \mathrm{E}$ & $1.8 \mathrm{E}-0$. \\
\hline n- & 10 & $1.7 E$ & $6.3 E-05$ \\
\hline SB126m & $19 \mathrm{Min}$. & $1.7 E-05$ & $6.3 E-05$ \\
\hline & ys & 5 & $5.6 \mathrm{E}-0$ \\
\hline & 6 & 5 & $3.9 E-$ \\
\hline & 2.3 & 6 & 3.2E -05 \\
\hline$m$ & 13. & 6 & $E-05$ \\
\hline PMt & rs & 6 & $E-05$ \\
\hline$G$ & 24 & 6 & $=-0.5$ \\
\hline $\mathrm{m}$ & 49.97 & 26 & -05 \\
\hline & & -06 & $=-06$ \\
\hline PD & 6 & 6 & 6 \\
\hline 3 & /s & 7 & 26 \\
\hline & $\dot{s}$ & 7 & 6 \\
\hline$m$ & 56.1 & 7 & $E-06$ \\
\hline & & 7 & $E-06$ \\
\hline it & is & $=-07$ & $1.2 E-06$ \\
\hline & 72 & -08 & 2.1E-07 \\
\hline CE & 32.51 Days & $2.7 E-08$ & $1.0 E-07$ \\
\hline & 60.2 Days & $3 E-08$ & 8.7E-OB \\
\hline im & 41.3 Days & $4 E-08$ & $5.5 E-08$ \\
\hline CDIISm & 44.59 Days & $.3 E-08$ & $4.8 E-08$ \\
\hline TE123m & 7 Days & $9 E-09$ & $1.1 E-08$ \\
\hline C 14 & $5.729 \mathrm{kY}$ & 2.7E- 09 & $1.0 E-08$ \\
\hline NB 94 & $20.3 \mathrm{kY}$ & $1.6 E-09$ & $5.9 E-09$ \\
\hline TE129m & 33.6 Day & $1.3 E-09$ & 4.9E-09 \\
\hline $\mathrm{HO} 166 \mathrm{~m}$ & $1.2 \mathrm{kY}$ & $1.1 E-09$ & $4.0 E-09$ \\
\hline TE12 & 1.16 Hours & $4 E-10$ & $3.2 E-09$ \\
\hline $\mathrm{M} 148$ & 5.37 Days & $E-10$ & $3.1 E-09$ \\
\hline CEI 42 & $104.9 \mathrm{GY}$ & $5 E-10$ & $2.5 E-09$ \\
\hline 387 & & $E-10$ & 2.2E-09 \\
\hline & 127 Year & $E-10$ & $8.5 E-10$ \\
\hline$S M 147$ & $107 \mathrm{GY}$ & $3 E-10$ & $5.0 E-10$ \\
\hline $3 E 10$ & $1.6 \mathrm{MY}$ & $E-11$ & $2.6 E-10$ \\
\hline J150 & 36 Years & $.9 E-11$ & $1.5 E-10$ \\
\hline & 2.37 Min. & $2.0 E-11$ & $7.5 E-11$ \\
\hline & 1.27 Years & $1.1 E-11$ & $4.3 E-11$ \\
\hline & 39.6 Sec. & $1.1 E-11$ & $4.3 E-11$ \\
\hline & 90000 & & $2.2 E-11$ \\
\hline & & & \\
\hline
\end{tabular}


Table 2.1. (contd)

Table 1-C. Decay Tirne $=10$ Years

\begin{tabular}{|c|c|c|c|}
\hline av & ate & Curies/kgU & PerCon \\
\hline & & & \\
\hline$\omega$ & $52 !$ & 1.828 & \\
\hline Y 90 & 6670 & 1.646 & 20.4 \\
\hline SA 90 & $.12 Y$ & 1.646 & \\
\hline PMI47 & 623 Years & 0.680 & \\
\hline 385 & $10.72 Y$ & 0.144 & 1.7 \\
\hline ki51 & 89.99 & 0.0414 & \\
\hline & & & 42 \\
\hline EU155 & $4.959 \mathrm{Y}$ & & \\
\hline Dines & & 21 & S \\
\hline RHI & 29.9 & & \\
\hline$\because 34$ & $2.062 Y$ & 99 & 0.123 \\
\hline 09144 & $1728 \mathrm{M}$ & & 0.121 \\
\hline CEI & 9 & 97 & 0.121 \\
\hline & & & \\
\hline & & & \\
\hline & & 0.0067 & 0.08 \\
\hline & & & $.5 E-0$ \\
\hline TC & $x_{1}$ & & \\
\hline PR1 & & 12 & \\
\hline$\cdots$ & & & \\
\hline EU1 & 13.6 & .05 & 4.8 \\
\hline & & & \\
\hline & & & \\
\hline 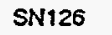 & 10 & & \\
\hline & & & \\
\hline Csi & 2. & -06 & $1.0 \mathrm{E}-0$ \\
\hline & & & \\
\hline sB & & & 2.5 \\
\hline 0 & & & \\
\hline & & & \\
\hline 112 & & .07 & 8.4 \\
\hline SN & & & \\
\hline & 249. & & $2.6 E-06$ \\
\hline & & & $7.4 E-07$ \\
\hline & & & \\
\hline 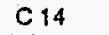 & 5.7 & -09 & -08 \\
\hline & & & \\
\hline & & .09 & \\
\hline & & & \\
\hline & & & \\
\hline 31 & & & -09 \\
\hline & & & \\
\hline & & & \\
\hline & & & \\
\hline & & & -09 \\
\hline & & & \\
\hline & & & \\
\hline & & & -10 \\
\hline 1 & $37+2$ & & \\
\hline & & & \\
\hline & & & \\
\hline & & & \\
\hline & & & \\
\hline & & & \\
\hline & & & \\
\hline & & & \\
\hline & & & \\
\hline & & & -14 \\
\hline & & & \\
\hline & & & \\
\hline & & & \\
\hline & & & \\
\hline & & & \\
\hline & & & \\
\hline & & & \\
\hline & $1 E+0$ & & \\
\hline & & & \\
\hline & $120.0 \mathrm{day}$ & & $1.15-11$ \\
\hline & & $2 E-19$ & $20=0$ \\
\hline
\end{tabular}

Table :-D. Docay Time $=30$ Years.

\begin{tabular}{|c|c|c|c|}
\hline 1801000 & Hat-Lito & Curios/kgu & Por Cont \\
\hline CS137 & 30 Yoars & 1.217 & 27.06 \\
\hline BA137m & 2.552 Min. & 1.151 & 25.60 \\
\hline Y 90 & 2.667 Days & 1.023 & 22.75 \\
\hline SR 90 & 29.12 Yoars & 1.022 & 22.73 \\
\hline KR 85 & 10.72 Years & 0.0394 & 0.876 \\
\hline SM151 & 89.99 Yoars & 0.0355 & 0.789 \\
\hline PM147 & 2.623 Yoars & 0.0034 & 0.077 \\
\hline $\mathrm{H}_{3}$ & 12.35 Years & 0.0023 & 0.051 \\
\hline EU154 & 8.6 Years & 0.0013 & 0.030 \\
\hline EU155 & 4.959 Years & 0.00097 & 0.022 \\
\hline TC 99 & $213 \mathrm{kY}$ & 0.00034 & $7.6 E-03$ \\
\hline CD113m & 14.59 Yoars & 0.00023 & $5.2 E-03$ \\
\hline SB125 & 2.77 Years & 0.00023 & $5.1 E-03$ \\
\hline TE125m & 58 Days & $5.6 \mathrm{E}-05$ & $1.2 E-03$ \\
\hline ZA 93 & $1.53 \mathrm{MY}$ & $4.7 E-05$ & $1.0 E-03$ \\
\hline NB 93m & 13.6 Yoars & $3.5 \mathrm{E}-05$ & $7.8 E-04$ \\
\hline $\mathrm{SB126m}$ & $19 \mathrm{Min}$. & $1.6 E-05$ & 3.7E-04 \\
\hline SN126 & $100 \mathrm{KY}$ & $1.6 E-05$ & $3.7 E-04$ \\
\hline EU152 & 13.6 Years & $1.4 E-05$ & $3.1 E-04$ \\
\hline CS134 & 2.062 Years & $1.2 E-05$ & $2.7 E-04$ \\
\hline SE 79 & $64.96 \mathrm{kY}$ & $1.0 E-05$ & $2.3 E-04$ \\
\hline CS135 & $2.3 \mathrm{Mr}$ & 8.3E-06 & $1.9 E-04$ \\
\hline SB126 & 12.4 Days & $2.3 E-06$ & $5.1 E-05$ \\
\hline SN121m & 49.97 Years & $2.0 \mathrm{E}-06$ & $4.5 E-05$ \\
\hline P0107 & $6.496 \mathrm{MY}$ & $1.2 E-06$ & $2.7 E-05$ \\
\hline 1129 & $15.7 \mathrm{MY}$ & 6.8E- -07 & $1.5 E-05$ \\
\hline PM146 & 5.5 Years & $1.3 E-07$ & $2.9 E-06$ \\
\hline $\mathrm{RH} 106$ & $29.9 \mathrm{Sec}$. & $1.3 E-08$ & $2.9 E-07$ \\
\hline RU106 & 1.008 Years & $1.3 E-08$ & $2.9 E-07$ \\
\hline$C_{14}$ & $5.729 \mathrm{kY}$ & $2.7 E-09$ & $6.0 E-08$ \\
\hline NB 94 & $20.3 \mathrm{kY}$ & $1.6 E-09$ & 3.4E-08 \\
\hline HO166m & $1.2 \mathrm{kY}$ & $1.0 E-09$ & $2.3 E-08$ \\
\hline CE142 & $104.9 \mathrm{GY}$ & $6.5 E-10$ & $1.4 E-08$ \\
\hline$R 887$ & $46.96 \mathrm{GY}$ & $5.9 E-10$ & $1.3 E-08$ \\
\hline $\mathrm{RH} 102$ & 2.9 Years & $5.0 E-10$ & 1.1E-OB \\
\hline SM: 47 & $107 \mathrm{GY}$ & $2.4 E-10$ & 5.3E-09 \\
\hline$A G 108 m$ & 127 Years & $1.9 E-10$ & $4.3 E-09$ \\
\hline PR144 & $17.28 \mathrm{Min}$. & $1.8 E-10$ & $4.0 E-09$ \\
\hline CE144 & 284.3 Days & $1.8 E-10$ & $4.0 \mathrm{E}-09$ \\
\hline $\mathrm{BE} 10$ & $1.6 \mathrm{MY}$ & $6.7 E-11$ & $1.5 E-09$ \\
\hline EU150 & 36 Years & $2.3 E-11$ & $5.1 E-10$ \\
\hline AG108 & 2.37 Min. & $1.7 E-11$ & $3.8 \mathrm{E}-10$ \\
\hline TC 98 & $4.199 \mathrm{MV}$ & $5.9 E-12$ & $1.3 E-10$ \\
\hline KA 81 & $209.9 \mathrm{kY}$ & $2.3 E-12$ & $5.2 E-11$ \\
\hline PR144m & 7.2 Min. & $2.1 \mathrm{E}-12$ & $4.8 E-11$ \\
\hline SM146 & $70 \mathrm{NH}$ & $1.8 E-13$ & $4.0 E-12$ \\
\hline ND144 & $2 E+06 \mathrm{GY}$ & $3.0 E-14$ & $6.6 E-13$ \\
\hline LA138 & $135 \mathrm{GY}$ & $4.2 E-15$ & 9.3E-14 \\
\hline NN115 & $5 E+05 \mathrm{GY}$ & $1.3 \varepsilon-15$ & $2.9 E-14$ \\
\hline SN119m & 245 Days & $4.1 E-16$ & $9.1 E-15$ \\
\hline$A G 110 \mathrm{~m}$ & 249.9 Days & $3.3 E-16$ & 7.3E-15 \\
\hline SM149 & $1 \bar{E}+07 \mathrm{GY}$ & $1.8 E-16$ & $4.1 E-15$ \\
\hline SM148 & $8 E+06 \mathrm{GY}$ & $1.4 E-16$ & $3.2 E-15$ \\
\hline TM171 & 1.92 Years & $5.0 E-18$ & $1.1 E-16$ \\
\hline AG109m & 39.6 Sec. & $4.5 E-18$ & $1.0 E-16$ \\
\hline CD109 & 1.27 Years & $4.5 E-18$ & $1.0 E-16$ \\
\hline$A G 110$ & 24.6 Sec. & $4.3 E-18$ & $9.7 E-17$ \\
\hline G0152 & $1 E+05 G Y$ & $4.2 E-18$ & $9.3 E-17$ \\
\hline GD153 & 242 Days & $5.0 E-19$ & $1.1 E-17$ \\
\hline TE123 & $10001 \mathrm{GY}$ & $2.2 \bar{E}-19$ & $5.0 E-18$ \\
\hline SN123 & 129.2 Days & $1.0 E-26$ & $2.2 \mathrm{E}-25$ \\
\hline $\mathrm{TE} 127 \mathrm{~m}$ & 109 Days & $6.7 E-31$ & $1.5 E-29$ \\
\hline TE127 & 9.35 Hours & $6.6 E-31$ & $1.5 E-29$ \\
\hline TE123m & 119.7 Days & $4.6 E-34$ & $1.0 E-32$ \\
\hline $7 N 17$ & 128.6 Days & $7.3 E-36$ & $1.6 E-34$ \\
\hline SB139 & 0.172 Sec. & 0 & \\
\hline TE133 & $12.45 \mathrm{Min}$. & 0 & \\
\hline TE133m & 55.4 Min. & 0 & \\
\hline XE133 & 5.245 Days & 0 & \\
\hline 1133 & 20.8 Hours & 0 & \\
\hline
\end{tabular}


Table 2.1. (contd)

Table 1-E. Decay Time $=40$ Years

\begin{tabular}{|c|c|c|c|}
\hline otope & alf-Lifo & Curies/kgu & Con \\
\hline & 30 Years & 9659 & \\
\hline BA137m & - & .9138 & \\
\hline & & 61 & \\
\hline & & & \\
\hline & 89.99 Yoars & 28 & \\
\hline & $10.72 Y_{0}$ & 0.0206 & \\
\hline & 12.35 Years & 0.0013 & 0 \\
\hline & $8.6 Y$ & & \\
\hline & 213 & 0.00034 & $6.9 E-C$ \\
\hline PM1 & $\begin{array}{l}2.623 \mathrm{Y} \\
4.959 \mathrm{Y}\end{array}$ & $\begin{array}{l}0.00025 \\
0.00024\end{array}$ & $\begin{array}{l}6.9 \mathrm{E}-0 . \\
6.8 \mathrm{E}-0\end{array}$ \\
\hline & 14.59 Yoars & 0.00015 & $=$ \\
\hline & $1.53 \mathrm{~N}$ & 05 & \\
\hline & & & \\
\hline & 2.77 Years & & \\
\hline & $19 \mathrm{Mi}$ & 1.6E-05 & $4.6 \mathrm{E}$ \\
\hline$S N+26$ & $100 \mathrm{kY}$ & -05 & $\triangle A F$ \\
\hline & & -05 & \\
\hline & ars & -06 & $24 \mathrm{~F}$ \\
\hline & & -06 & $2.3 E$ \\
\hline & & -06 & \\
\hline & & & \\
\hline & 49. & $-\infty$ & 5.08 \\
\hline$P D$ & & & \\
\hline & & & \\
\hline$\because$ & 2.062 Years & 4.2E-07 & $1.2 E-C$ \\
\hline & & -08 & \\
\hline & & -09 & $7.6 \mathrm{E}$ \\
\hline & & -09 & $4.4 E-$ \\
\hline & & $-\infty 9$ & 2 \\
\hline $\mathrm{CE}$ & & -10 & $1.8 \mathrm{E}$ \\
\hline RE & & & 1.7 \\
\hline$S M$ & 10 & -10 & 6.78 \\
\hline & & & $=$ \\
\hline & & -11 & $1.9 \mathrm{E}-\mathrm{C}$ \\
\hline $\mathrm{RH}$ & & & $1.3 \mathrm{E}-\mathrm{C}$ \\
\hline & 3 & -11 & $5.3 E-1$ \\
\hline & $2.37 \mathrm{M}$ & -11 & $4.6 \mathrm{E}$ \\
\hline & & & $E$ \\
\hline & 1.0 & -11 & $3.8 E-1$ \\
\hline & & & \\
\hline & & & $=$ \\
\hline & $M$ & -13 & $2 E-$ \\
\hline & $2 \mathrm{E}$ & & 8.4E- \\
\hline & & $2.4 E-14$ & $6.8 \mathrm{E}-$ \\
\hline PR144 & Min & $2.4 E-14$ & $6.8 \mathrm{E}-$ \\
\hline & & -15 & $1.2 \mathrm{E}-1$ \\
\hline $\mathbb{N}$ & $5 E$ & -15 & (1) \\
\hline & & & $E-$ \\
\hline & & -16 & $5.1 E$ \\
\hline & & & 0 \\
\hline & & -18 & $1.2 \mathrm{E}-1$ \\
\hline & & & \\
\hline & & -19 & 4.1E-1 \\
\hline$A G$ & 39.6 & -20 & $5.5 \mathrm{E}$ \\
\hline & & & $3.8 \mathrm{E}-$ \\
\hline & $249.9 \mathrm{DE}$ & 1.3E-20 & $3.7 \mathrm{E}-$ \\
\hline & & & \\
\hline & & -22 & $4.9 \mathrm{E}-$ \\
\hline & 0 & $1.4 E-23$ & $4.0 \mathrm{E}-$ \\
\hline & 5 & & - \\
\hline & & & \\
\hline & & & \\
\hline & & & \\
\hline & $9 \mathrm{Mi}$ & & \\
\hline & $8.041 \mathrm{DE}$ & 0 & \\
\hline & $2.3 \mathrm{Ho}$ & & \\
\hline & 0.8 Hours & & \\
\hline & 9 So & & \\
\hline & $6 \mathrm{M}$ & & \\
\hline
\end{tabular}

Table 1-F. Decay Time $=100$ Years

\begin{tabular}{|c|c|c|c|}
\hline sotope & Half-Life & Curies/kgU & Por Cent \\
\hline 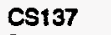 & 30 Yoars & 0.2415 & 27.50 \\
\hline BA137m & 2.552 Min. & 0.2284 & 26.01 \\
\hline Y90 & 2.667 Days & 0.1933 & 22.01 \\
\hline SR 90 & 29.12 Yoars & 0.1932 & 22.00 \\
\hline SM151 & 89.99 Yoars & 0.0207 & 2.36 \\
\hline KA 85 & 10.72 Years & 0.00043 & 0.049 \\
\hline TC 99 . & $213 \mathrm{kr}$ & 0.00034 & 0.039 \\
\hline ZR 93 & $1.53 \mathrm{MN}$ & $4.7 E-05$ & $5.4 E-03$ \\
\hline $\mathrm{H}_{3}$ & 12.35 Yoars & 4.5E-05 & $5.1 E-03$ \\
\hline NB 93m & 13.6 Years & $4.5 E-05$ & $5.1 E-03$ \\
\hline SN128 & $100 \mathrm{kY}$ & $1.6 \mathrm{E}-05$ & $1.9 E-03$ \\
\hline SB126m & 19 Min. & $1.6 E-05$ & $1.9 E-03$ \\
\hline SE 79 & $64.96 \mathrm{kY}$ & $1.0 E-05$ & $1.2 E-03$ \\
\hline CD113m & 14.59 Years & 8.4E-06 & $9.6 E-04$ \\
\hline CS135 & $2.3 \mathrm{MY}$ & 8.3E-06 & $9.5 \mathrm{E}-04$ \\
\hline EU154 & 8.6 Yoars & $4.8 E-06$ & $5.4 \mathrm{E}-04$ \\
\hline SB126 & 12.4 Days & $2.3 E-06$ & 2.6E-04 \\
\hline $\begin{array}{l}\text { PO107 } \\
\text { SN121m }\end{array}$ & $6.496 \mathrm{MY}$ & $1.2 \mathrm{E}-06$ & 1.4E-04 \\
\hline $\begin{array}{l}\text { SN121m } \\
1129\end{array}$ & $\begin{array}{l}49.97 \text { Years } \\
15.7 \mathrm{MY}\end{array}$ & $\begin{array}{l}7.7 E-07 \\
6.8 E-07\end{array}$ & $\begin{array}{l}8.7 E-05 \\
7.7 E-05\end{array}$ \\
\hline EU152 & 13.6 Yoars & $3.9 E-07$ & $4.5 \mathrm{E}-05$ \\
\hline EU155 & 4.959 Years & $5.5 E-08$ & $6.3 \mathrm{E}-06$ \\
\hline C14 & $5.729 \mathrm{kY}$ & 2.7E-09 & 3.1E-07 \\
\hline NB 94 & $20.3 \mathrm{kY}$ & $1.5 E-09$ & $1.8 \mathrm{E}-07$ \\
\hline HO166m & $1.2 \mathrm{kY}$ & $9.9 E-10$ & $1.1 E-07$ \\
\hline CE142 & $104.9 \mathrm{GY}$ & $6.5 \bar{E}-10$ & $7.4 E-08$ \\
\hline $\mathrm{AB} 87$ & $46.96 \mathrm{GY}$ & $5.9 E-10$ & $6.7 E-08$ \\
\hline SM147 & $107 \mathrm{GY}$ & $2.4 E-10$ & 2.7E-08 \\
\hline AG108m & 127 Years & 1.3E-10 & $1.5 E-08$ \\
\hline BE 10 & $1.6 \mathrm{Mr}$ & $6.7 E-11$ & $7.7 \bar{E}-09$ \\
\hline PM147 & 2.623 Years & $3.2 E-11$ & $3.6 E-09$ \\
\hline PM146 & 5.5 Years & $1.9 E-11$ & $2.2 E-09$ \\
\hline AG108 & $2.37 \mathrm{Min}$. & $1.2 E-11$ & $1.3 E-09$ \\
\hline EU150 & 36 Years & $6.0 E-12$ & $6.8 E-10$ \\
\hline TC 98 & $4.199 \mathrm{Mr}$ & $5.9 E-12$ & $6.7 E-10$ \\
\hline SB125 & 2.77 Yoars & $5.7 E-12$ & $6.4 E-10$ \\
\hline KR 81 & $209.9 \mathrm{kY}$ & $2.3 E-12$ & $2.7 E-10$ \\
\hline TE125m & 58 Days & $1.4 \mathrm{E}-12$ & $1.6 \mathrm{E}-10$ \\
\hline SM146 & & $1.8 E-13$ & $2.1 E-11$ \\
\hline ND144 & $2 E+06 \mathrm{GY}$ & 3.OE-14 & $3.4 E-12$ \\
\hline LA138 & 135 Gy & $4.2 E-15$ & $4.8 E-13$ \\
\hline IN115 & $5 E+05 G Y$ & 1.3E-15 & $1.5 \mathrm{E}-13$ \\
\hline CS134 & 2.062 Years & $7.2 E-16$ & 8.2E -14 \\
\hline SM149 & $1 E+07 \mathrm{GY}$ & $1.8 E-16$ & $2.1 E-14$ \\
\hline SM148 & $8 E+06 \mathrm{GY}$ & $1.4 E-16$ & $1.7 E-14$ \\
\hline $\mathrm{AH} 102$ & 2.9 Yoars & $2.7 E-17$ & $3.1 E-15$ \\
\hline GD152 & $1 E+05 G Y$ & $4.7 E-18$ & $5.3 E-16$ \\
\hline TE123 & $1000 t \mathrm{GY}$ & $2.2 E-19$ & $2.5 E-17$ \\
\hline TM171 & 1.92 Yoars & $5.3 E-29$ & $6.0 E-27$ \\
\hline RH106 & 29.9 Sec. & $1.6 \mathrm{E}-29$ & 1.8E-27 \\
\hline RU106 & 1.008 Years & $1.6 E-29$ & $1.8 E-27$ \\
\hline CD109 & 1.27 Years & $1.2 \mathrm{E}-34$ & $1.3 E-32$ \\
\hline CS133 & 0 Stable & & \\
\hline BA133 & 4 Yoars & 0 & \\
\hline XE133m & 2.19 Days & 0 & \\
\hline IN134 & 0.078 Sec. & 0 & \\
\hline XE133 & 5.245 Days & 0 & \\
\hline $1133 m$ & 9 Sec. & 0 & \\
\hline S8134 & 11 Sec. & 0 & \\
\hline XE131m & 11.9 Days & 0 & \\
\hline 1931 & 8.041 Days & 0 & \\
\hline $\mathbb{N} 132$ & 0.12 Sec. & 0 & \\
\hline CD132 & 0.145 Sec. & 0 & \\
\hline$S 8132$ & $2.8 \mathrm{Min}$. & 0 & \\
\hline XE131 & O Stable & 0 & \\
\hline TE132 & 3.258 Days & 0 & \\
\hline TE131m & 1.25 Days & 0 & \\
\hline TE131 & $25 \mathrm{Min}$. & 0 & \\
\hline SN132 & 40 Sec. & 0 & \\
\hline 1133 & 20.8 Hours & 0 & \\
\hline
\end{tabular}


Table 2.1. (contd)

Table 1-G. Decay Time $=300$ Years

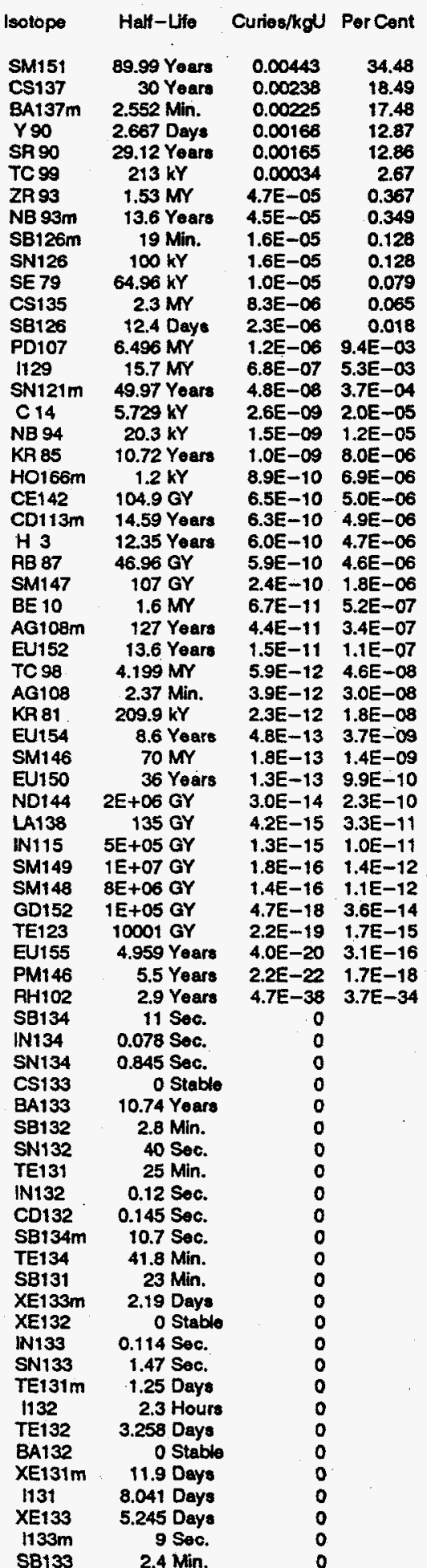

Table $1-\mathrm{H}$. Decay Time $=1000$ Years

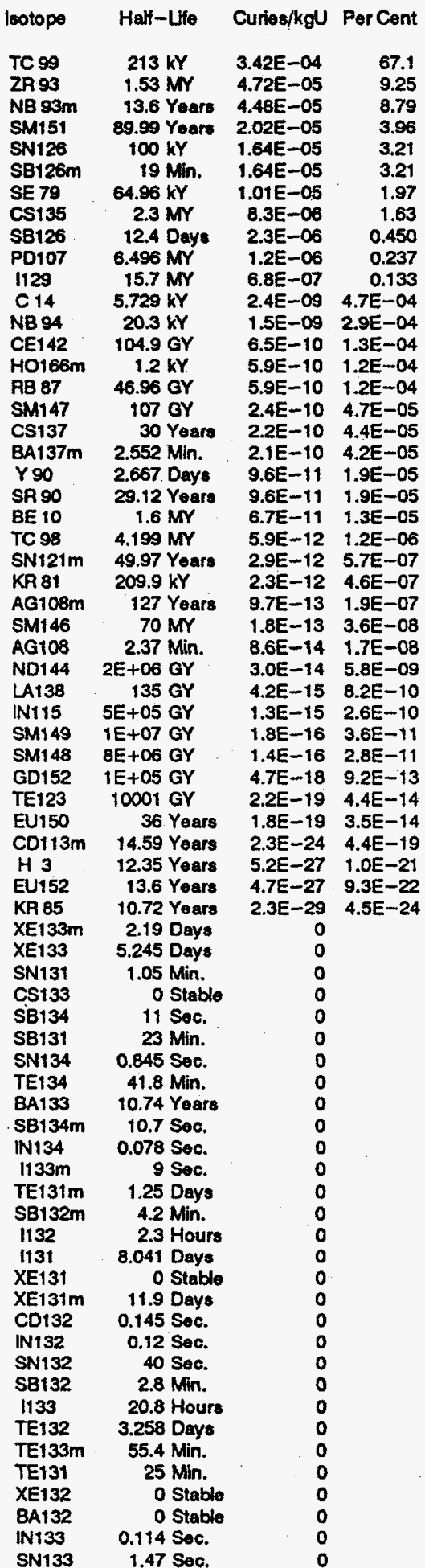


Table 2.1. (contd)

Table 1-1. Decay Time $=3000$ Yoars

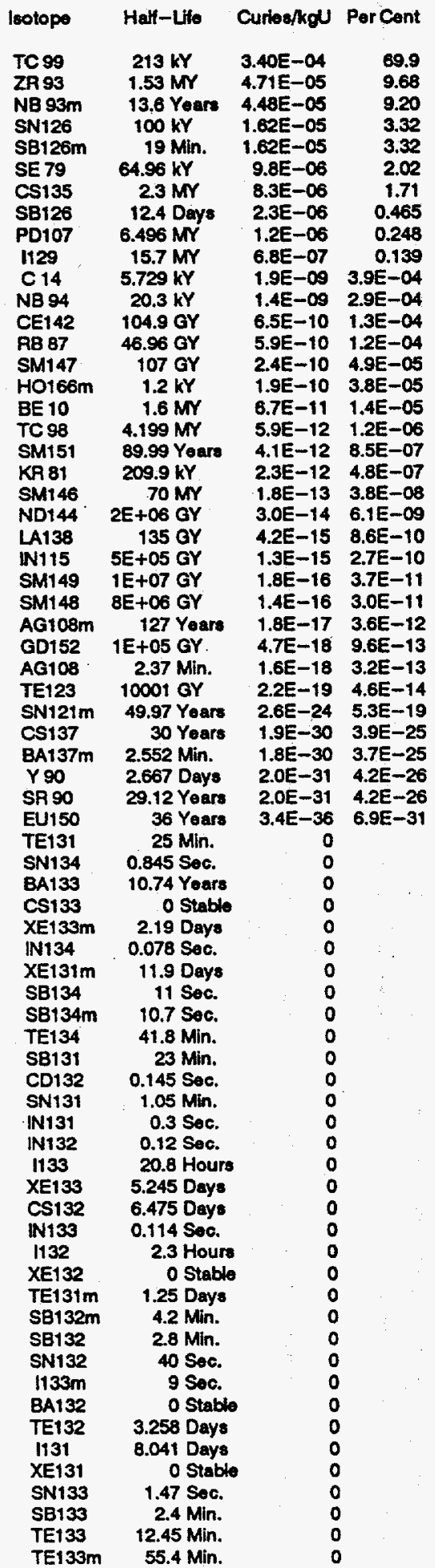


Table 2.2. Radionuclide Content of Activation Products in Hanford Reactor Fuel and Cladding at Various Decay Times

Table 2-A. Decay Time 1 Year

\begin{tabular}{|c|c|c|c|}
\hline otope & Hali-Lifo & Curies/kgu & Per Cont \\
\hline $\begin{array}{l}\text { H } 3 \\
\text { FE } 55\end{array}$ & $\begin{array}{r}12.35 \text { Years } \\
2.6 \text { Years }\end{array}$ & $\begin{array}{l}0.0739 \\
0.0267\end{array}$ & $\begin{array}{l}51.42 \\
18.57\end{array}$ \\
\hline N895 & 35.15 Days & 0.0144 & 10.03 \\
\hline & 63.98 Days & 0.0067 & 4.65 \\
\hline SN119m & 245 Days & 0.0064 & 4.47 \\
\hline & 70.78 Days & 0.0039 & 2.69 \\
\hline$\infty 060$ & 5.270 Ye ars & 0.0027 & 1.85 \\
\hline$\sqrt{63}$ & 92 Years & 0.0023 & 1.58 \\
\hline$B 125$ & 2.77 Years & 0.0021 & 1.46 \\
\hline AN 54 & 312.5 Days & 0.0013 & 0.877 \\
\hline $113 \mathrm{~m}$ & 1.658 Hours & 0.0011 & 0.777 \\
\hline SNI13 & 115.1 Days & 0.0011 & 0.776 \\
\hline TE125m & 58 Days & 0.00051 & 0.352 \\
\hline SN123 & 129.2 Deye & 0.00036 & 0.253 \\
\hline C 14 & 5.7 & 0.00012 & 0.087 \\
\hline B 95m & 3.60 & 5.08 & 0.034 \\
\hline F181 & bays & -05 & 0.022 \\
\hline 1 & 27.7 & -05 & 0.018 \\
\hline 5185 & ays & 5 & 0.017 \\
\hline Y91 & 58.51 Days & -05 & 0.013 \\
\hline 15 & 80 & $1.8 E-05$ & 0.013 \\
\hline 55 & ays & $1.7 E-05$ & 0.012 \\
\hline WIBS & 75.1 Days & $1.6 \mathrm{E}-05$ & 0.011 \\
\hline 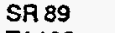 & 50.5 & $3.9 E-06$ & $2.7 E-03$ \\
\hline IA & ays & $3.8 E-06$ & $2.7 E-03$ \\
\hline CD109 & 1.27 Years & $2.9 E-06$ & $2.0 E-03$ \\
\hline $9 \mathrm{~m}$ & 39.6 Sec. & $2.9 E-06$ & 2.0E-03 \\
\hline 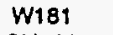 & 121.2 Days & $1.9 E-06$ & $1.3 E-03$ \\
\hline SN121m & 49.97 Years & $1.3 \bar{E}-06$ & $9.2 E-04$ \\
\hline$N 6$ & 243.9 Days & $1.2 E-06$ & $8.1 E-04$ \\
\hline $0115 \mathrm{~m}$ & 44.59 Deys & $1.1 E-06$ & $7.5 E-04$ \\
\hline 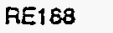 & 16.98 Hours & $5.1 E-07$ & $3.6 E-04$ \\
\hline W188 & 69.4 Days & 5.1E-07 & $3.5 E-04$ \\
\hline $1114 \mathrm{~m}$ & 49.51 Days & $2.8 E-07$ & $1.9 E-04$ \\
\hline 114. & $1.198 \mathrm{Min}$. & $2.7 E-07$ & $1.9 E-04$ \\
\hline 93 & $1.530 \mathrm{Mr}$ & $2.2 E-07$ & $1.5 E-04$ \\
\hline 46 & 83.80 Days & $1.8 E-07$ & $1.2 E-04$ \\
\hline 3124 & 60.2 Days & $3.2 E-08$ & 2.2E-05 \\
\hline $93 m$ & 13.60 Years & $1.2 E-08$ & $8.2 E-06$ \\
\hline $123 \mathrm{~m}$ & 139.7 Days & $4.7 E-09$ & $3.3 E-06$ \\
\hline 45 & 163.0 Days & $3.7 E-09$ & $2.6 E-06$ \\
\hline $1117 \mathrm{~m}$ & 14 Days & $1.5 E-09$ & $1.0 E-06$ \\
\hline 93 & $3.498 \mathrm{kY}$ & $1.4 E-09$ & $9.6 E-07$ \\
\hline 10 & $i .60$ & $2.7 E-10$ & $1.9 E-07$ \\
\hline & 2.667 & $2.4 E-10$ & $1.7 E-07$ \\
\hline & 29.12 & $2.4 E-10$ & $1.7 E-07$ \\
\hline Om & 249.9 Days & $1.8 E-10$ & $1.3 E-07$ \\
\hline & 21 & $6.5 E-11$ & 4.5E-08 \\
\hline & ays & $5 E-11$ & $2.5 E-08$ \\
\hline & 109 Days & $1.3 E-11$ & $8.8 E-09$ \\
\hline & 9.35 Hours & $1.2 E-11$ & -09 \\
\hline & 6.70 & $8.2 E-12$ & $5.7 E-09$ \\
\hline $3 m$ & 127 Years & $7.3 E-12$ & $5.0 E-09$ \\
\hline & & $2.4 E-12$ & $1.7 E-09$ \\
\hline & 20. & 2.3E-12 & $1.6 \mathrm{E}-09$ \\
\hline & 2.37 Min. & $6.5 E-13$ & $4.5 E-10$ \\
\hline 18 & $9 \mathrm{MY}$ & $5.3 E-13$ & $3.7 E-10$ \\
\hline & 14. & $5.1 E-13$ & $3.6 \mathrm{E}-10$ \\
\hline & 650 Years & $5.1 E-13$ & $3.5 E-10$ \\
\hline 187 & $50 \mathrm{GY}$ & $8.8 E-14$ & $6.1 E-11$ \\
\hline & 9.64 Days & $8.2 E-14$ & $5.7 E-11$ \\
\hline 205 & $30 \mathrm{Mr}$ & B.OE-14 & $5.6 E-11$ \\
\hline 103 & 39.28 Days & $5.7 E-14$ & $3.9 E-11$ \\
\hline 210 & 138.4 Days & $4.7 E-14$ & $3.3 E-11$ \\
\hline 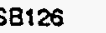 & 12.4 Deys & $3.6 E-14$ & $2.5 E-11$ \\
\hline & 74.02 Days & $5.5 E-16$ & $3.8 E-13$ \\
\hline & 10.16 Days & $4.0 E-17$ & $2.8 E-14$ \\
\hline & & $1.2 E-17$ & $8.2 E-15$ \\
\hline & $4.199 \mathrm{MY}$ & $1.3 E-18$ & $9.1 E-16$ \\
\hline 127 & 36.41 Days & $2.4 \mathrm{E}-19$ & $1.7 E-16$ \\
\hline
\end{tabular}

Table 2-8. Decay Time $=3$ Years

\begin{tabular}{|c|c|c|c|}
\hline Isolope & Hah-Lifo & Curies/kgU & Per Cent \\
\hline $\begin{array}{l}\mathrm{H} 3 \\
\text { FE } 55\end{array}$ & $\begin{array}{r}12.35 \text { Yoars } \\
2.6 \text { Yoars }\end{array}$ & $\begin{array}{l}0.0660 \\
0.0157\end{array}$ & $\begin{array}{l}74.36 \\
17.63\end{array}$ \\
\hline$N ! 53$ & 92 Years & 0.0022 & 2.52 \\
\hline 0060 & 5.270 Years & 0.0020 & 2.29 \\
\hline SBI25 & 2.77 Years & 0.0013 & 1.43 \\
\hline SN119m & 245 Dayo & 0.00081 & 0.916 \\
\hline TE125m & 58 Days & 0.00031 & 0.349 \\
\hline MN 54 & 312.5 Days & 0.00025 & 0.281 \\
\hline$C_{14}$ & $5.729 \mathrm{kY}$ & 0.00012 & 0.140 \\
\hline NI 59 & $80 \mathrm{KY}$ & $1.8 E-05$ & 0.021 \\
\hline IN113m & 1.658 Houts & $1.4 E-05$ & 0.015 \\
\hline SN113 & 115.1 Days & $1.4 \mathrm{E}-05$ & 0.015 \\
\hline SN123 & 129.2 Days & $7.2 E-06$ & $8.1 E-03$ \\
\hline NB 95 & 35.15 Days & $5.4 E-06$ & 6.1E-03 \\
\hline CO 58 & 70.78 Days & $3.0 \mathrm{E}-06$ & $3.4 E-03$ \\
\hline ZF 95 & 63.98 Days & $2.4 E-06$ & $2.7 E-03$ \\
\hline SN121m & 49.97 Years & $1.3 E-\infty 6$ & $1.5 E-03$ \\
\hline AG109m & 39.6 Soc. & $9.7 E-07$ & $1.1 E-03$ \\
\hline CD109 & 1.27 Years & $9.7 E-07$ & $1.1 E-03$ \\
\hline ZR 93 & $1.530 \mathrm{Mr}$ & $2.2 E-07$ & 2.5E-04 \\
\hline ZN 65 & 243.9 Days & $1.5 E-07$ & $1.6 \mathrm{E}-04$ \\
\hline TA182 & 115 Days & 4.7E-08 & 5.3E-05 \\
\hline NB 93m & 13.60 Years & $3.1 E-08$ & $3.5 E-05$ \\
\hline W181 & 121.2 Days & $2.9 E-08$ & $3.3 E-05$ \\
\hline W185 & 75.1 Days & $1.9 E-08$ & $2.2 E-05$ \\
\hline NB $95 \mathrm{~m}$ & 3.609 Days & $1.8 E-08$ & $2.0 E-05$ \\
\hline HF175 & 70 Days & $1.7 E-08$ & 2.0E-05 \\
\hline Y 91 & 58.51 Days & $3.2 E-09$ & $3.7 E-06$ \\
\hline MO 93 & $3.498 \mathrm{kY}$ & $1.4 E-09$ & $1.6 E-06$ \\
\hline $\operatorname{SC~} 46$ & 83.80 Days & $4.3 E-10$ & 4.8E-07 \\
\hline RE188 & 16.98 Hours & $3.5 E-10$ & $3.9 E-07$ \\
\hline W18B & 69.4 Days & $3.5 E-10$ & $3.9 E-07$ \\
\hline BE 10 & $1.600 \mathrm{MN}$ & $2.7 E-10$ & $3.1 E-07$ \\
\hline SR 90 & 29.12 Years & $2.3 E-10$ & 2.6E-07 \\
\hline$Y 90$ & 2.667 Days & $2.3 E-10$ & $2.6 E-07$ \\
\hline FE 59 & 45 Days & $2.2 E-10$ & $2.5 E-07$ \\
\hline HF181 & 42.4 Days & $2.1 E-10$ & $2.3 E-07$ \\
\hline SR 89 & 50.50 Days & $1.7 E-10$ & $2.0 E-07$ \\
\hline CA 45 & 163.0 Days & $1.7 E-10$ & $1.9 \mathrm{E}-07$ \\
\hline TE123m & 119.7 Days & $6.8 E-11$ & 7.7E-08 \\
\hline TC 99 & $213 \mathrm{kY}$ & $6.5 E-11$ & 7.3E-08 \\
\hline$A G 110 \mathrm{~m}$ & 249.9 Days & $2.4 E-11$ & $2.7 E-08$ \\
\hline CD115m & 44.59 Days & $1.3 E-11$ & $1.4 \mathrm{E}-08$ \\
\hline NI $14 \mathrm{~m}$ & 49.51 Days & $1.0 E-11$ & $1.1 E-08$ \\
\hline IN114 & $1.198 \mathrm{Min}$. & $9.6 E-12$ & $1.1 E-08$ \\
\hline AG108m & 127 Years & $7.2 E-12$ & $8.1 E-09$ \\
\hline SB124 & 60.2 Days & $7.0 E-12$ & $7.9 E-09$ \\
\hline NB 94 & $20.3 \mathrm{kY}$ & $2.3 E-12$ & $2.6 E-09$ \\
\hline LU177m & 155 Days & $1.4 \mathrm{E}-12$ & $1.5 E-09$ \\
\hline AG108 & 2.37 Min. & $6.4 E-13$ & $7.2 E-10$ \\
\hline HF182 & $9 \mathrm{Mr}$ & $5.3 E-13$ & $6.0 E-10$ \\
\hline S1 32 & 650 Years & $5.1 E-13$ & $5.7 E-10$ \\
\hline P 32 & 14.3 Days & $5.1 E-13$ & $5.7 E-10$ \\
\hline AG110 & 24.6 Sec. & $3.2 E-13$ & $3.6 \mathrm{E}-10$ \\
\hline LU17T & 6.709 Days & $3.1 \mathrm{E}-13$ & $3.5 E-10$ \\
\hline CR 51 & 27.71 Days & $3.0 E-13$ & $3.4 E-10$ \\
\hline TE127m & 109 Days & $1.2 E-13$ & $1.4 \mathrm{E}-10$ \\
\hline TE127 & 9.35 Hours & $1.2 E-13$ & $1.3 E-10$ \\
\hline RE 187 & $50 \mathrm{GY}$ & $8.8 E-14$ & $9.9 E-11$ \\
\hline P8205 & $30 \mathrm{MY}$ & $8.0 E-14$ & $9.1 E-11$ \\
\hline PO210 & 138.4 Days & $1.2 E-15$ & $1.4 E-12$ \\
\hline LU176 & $30 \mathrm{GY}$ & $1.2 E-17$ & $1.3 E-14$ \\
\hline TC 98 & $4.199 \mathrm{MV}$ & $1.3 E-18$ & $1.5 E-15$ \\
\hline IR192 & 74.02 Days & $5.2 E-19$ & $5.9 E-16$ \\
\hline V 50 & $4 E+07 \mathrm{GY}$ & $1.7 E-19$ & $1.9 E-16$ \\
\hline RU103 & 39.26 Days & $1.4 E-19$ & $1.6 E-16$. \\
\hline PB204 & $1 E+08 \mathrm{GY}$ & $1.3 E-19$ & $1.5 E-16$ \\
\hline IN115 & $5 E+05 \mathrm{GY}$ & $1.2 E-19$ & $1.4 E-16$ \\
\hline $\mathrm{B} 1208$ & $368 \mathrm{kY}$ & $4.7 E-20$ & $5.3 E-17$ \\
\hline $\mathrm{B} 1210 \mathrm{~m}$ & $3 M Y$ & $3.1 E-20$ & $3.5 E-17$ \\
\hline
\end{tabular}


Table 2.2. (contd)

Table 2-C. Decay Time $=10$ Yoars

\begin{tabular}{|c|c|c|c|}
\hline Isotope & Hall-Lito & Curies/kgu & Por Cent \\
\hline H 3 & 12.35 Yoaps & 0.04456 & 88.53 \\
\hline FE 55 & 2.6 Years & 0.00242 & 4.81 \\
\hline NI 63 & 92 Years & 0.00212 & 4.22 \\
\hline $\mathrm{CO} 60$ & 5.270 Yoars & 0.00081 & 1.51 \\
\hline SB125 & 2.77 Years & 0.00022 & 0.437 \\
\hline C 14 & $5.729 \mathrm{kY}$ & 0.00012 & 0.248 \\
\hline TE125m & 58 Days & $5.4 E-05$ & 0.107 \\
\hline NI 59 & $80 \mathrm{kY}$ & 1.8E-05 & 0.037 \\
\hline SN121m & 49.97 Years & $1.2 E-\infty 6$ & $2.3 E-03$ \\
\hline MN 54 & 312.5 Days & $8.6 E-07$ & $1.7 E-03$ \\
\hline SN119m & 245 Days & $5.9 E-07$ & $1.2 E-03$ \\
\hline ZR 93 & $1.530 \mathrm{MY}$ & $2.2 E-07$ & $4.3 E-04$ \\
\hline NB 93m & 13.60 Years & $8.4 E-08$ & $1.7 E-04$ \\
\hline AG109m & 39.6 Sec. & $2.1 E-08$ & $4.2 E-05$ \\
\hline CDi09 & 1.27 Years & $2.1 E-08$ & $4.2 E-05$ \\
\hline MO 93 & $3.498 \mathrm{kY}$ & $1.4 E-09$ & $2.8 E-06$ \\
\hline BE 10 & $1.600 \mathrm{MY}$ & $2.7 E-10$ & 5.4E-07 \\
\hline SR90 & 29.12 Years & $2.0 E-10$ & $3.9 E-07$ \\
\hline Y90 & 2.667 Days & $2.0 E-10$ & $3.9 E-07$ \\
\hline ZN 65 & 243.9 Days & $1.0 E-10$ & $2.0 E-07$ \\
\hline TC 99 & $213 k Y$ & $6.5 E-11$ & $1.3 E-07$ \\
\hline SN123 & 129.2 Days & $7.9 E-12$ & $1.6 E-08$ \\
\hline AG108m & 127 Years & $6.9 E-12$ & $1.4 E-08$ \\
\hline $\mathbb{N} 113 \mathrm{~m}$ & 1.658 Hours & $2.8 E-12$ & $5.6 \mathrm{E}-09$ \\
\hline SN113 & 115.1 Days & 2. $8 E-12$ & $5.6 \mathrm{E}-09$ \\
\hline NB 94 & $20.3 \mathrm{kY}$ & $2.3 E-12$ & $4.5 E-09$ \\
\hline AG10B & 2.37 Min. & $6.1 E-13$ & $1.2 E-09$ \\
\hline TA182 & 115 Days & $5.4 E-13$ & $1.1 E-09$ \\
\hline HFI82 & $9 \mathrm{inY}$ & $5.3 E-13$ & $1.1 E-09$ \\
\hline P 32 & 14.3 Days & $5.0 E-13$ & $1.0 E-09$ \\
\hline S1 32 & 650 Years & $5.0 E-13$ & $1.0 E-09$ \\
\hline RE187 & $50 \mathrm{GY}$ & $8.8 E-14$ & $1.7 E-10$ \\
\hline PB205 & $30 M Y$ & $8.0 E-14$ & $1.6 \mathrm{E}-10$ \\
\hline AG110m & 249.9 Days & $2.0 E-14$ & $3.9 E-11$ \\
\hline W181 & 121.2 Days & $1.3 E-14$ & $2.6 E-11$ \\
\hline CA 45 & 163.0 Days & $3.2 E-15$ & $6.3 E-12$ \\
\hline AG110 & 24.6 Sec. & $2.6 \mathrm{E}-16$ & $5.2 E-13$ \\
\hline CO 58 & 70.78 Days & $4.0 E-17$ & $8.0 E-14$ \\
\hline TE123m & 119.7 Days & $2.5 E-17$ & $5.0 E-14$ \\
\hline LU177m & 155 Days & $1.5 E-17$ & $2.9 E-14$ \\
\hline LU176 & $30 \mathrm{GY}$ & $1.2 E-17$ & $2.3 E-14$ \\
\hline NB 95 & 35.15 Days & $5.1 E-18$ & $1.0 E-14$ \\
\hline LU177 & 6.709 Days & $3.4 E-18$ & $6.7 E-15$ \\
\hline ZR 95 & 63.98 Days & $2.3 E-18$ & $4.5 E-15$ \\
\hline TC 98 & $4.199 \mathrm{Mr}$ & $1.3 E-18$ & $2.6 E-15$ \\
\hline W185 & 75.1 Days & $1.1 E-18$ & $2.2 E-15$ \\
\hline $\operatorname{SC~} 46$ & 83.80 Days & $2.8 E-19$ & $5.5 E-16$ \\
\hline HFi75 & 70 Days & $1.8 E-19$ & $3.5 E-16$ \\
\hline V 50 & $4 E+07 G Y$ & $1.7 E-19$ & $3.4 E-16$ \\
\hline $\mathrm{PB2O4}$ & $1 \mathrm{E}+08 \mathrm{GY}$ & $1.3 E-19$ & $2.6 E-16$ \\
\hline IN115 & $5 E+05 G Y$ & $1.2 E-19$ & $2.5 E-16$ \\
\hline $\mathrm{B} 1208$ & $368 \mathrm{kY}$ & 4.7E-20 & $9.4 E-17$ \\
\hline $81210 \mathrm{~m}$ & $3 M Y$ & $3.1 E-20$ & $6.2 E-17$ \\
\hline T2206 & 4.19 Min. & $3.1 E-20$ & $6.2 E-17$ \\
\hline NB 95m & 3.609 Days & $1.7 E-20$ & $3.4 E-17$ \\
\hline TE127m & 109 Days & $1.1 E-20$ & $2.1 E-17$ \\
\hline TE127 & 9.35 Hours & $1.0 E-20$ & $2.0 E-17$ \\
\hline PD107 & $6.496 \mathrm{Mr}$ & $7.1 E-21$ & $1.4 E-17$ \\
\hline IA192 & 74.02 Days & $6.7 E-21$ & $1.3 E-17$ \\
\hline IA192m & 241 Years & $6.7 E-21$ & $1.3 E-17$ \\
\hline TE123 & $10001 \mathrm{GY}$ & $5.3 E-21$ & $1.1 E-17$ \\
\hline PO210 & 138.4 Days & $3.5 E-21$ & $6.9 E-18$ \\
\hline RE188 & 16.98 Hours & $2.8 E-21$ & $5.6 E-18$ \\
\hline W188 & 69.4 Days & $2.8 E-21$ & $5.6 E-18$ \\
\hline $\mathrm{RH} 102$ & 2.9 Years & $1.6 E-21$ & $3.2 E-18$ \\
\hline PT193 & 500 Years & $4.0 E-22$ & $7.9 E-19$ \\
\hline Y91 & 58.51 Days & $2.3 E-22$ & $4.5 E-19$ \\
\hline SB124 & 60.2 Days & $1.1 E-24$ & 2.3E-21 \\
\hline 1129 & $15.7 \mathrm{MY}$ & $1.2 E-25$ & $2.3 E-22$ \\
\hline SR 89 & 50.50 Days & $9.9 E-26$ & $2.0 E-22$ \\
\hline
\end{tabular}

Table 2-D. Decay Time $=30$ Years

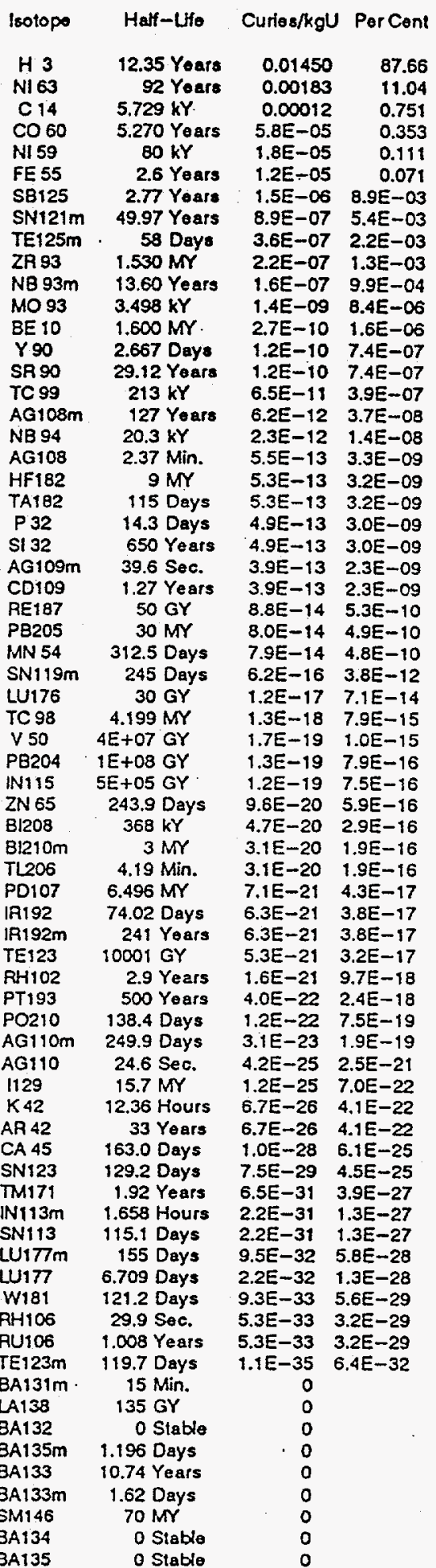


Table 2.2. (contd)

Table 2-E. Decay Time $=40$ Years

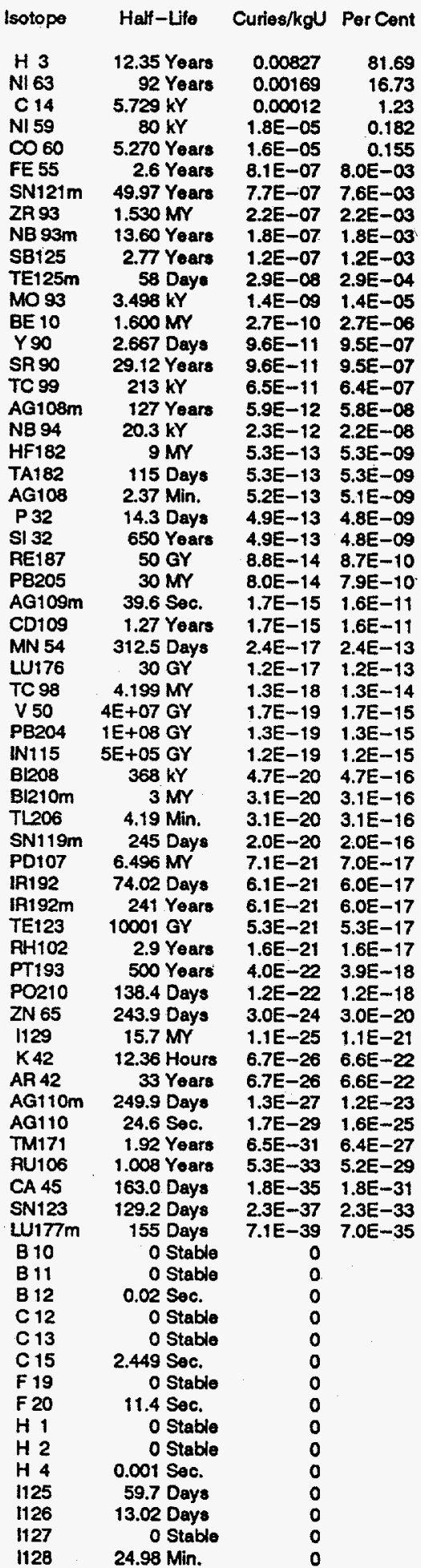

Table 2-F. Decay Time $=100$ Years

\begin{tabular}{|c|c|c|c|}
\hline Isotope & Half-Life & Curies/kgU & Per Cent \\
\hline N1 63 & 92 Years & 0.001078 & 71.6 \\
\hline H 3 & 12.35 Years & 0.000285 & 18.9 \\
\hline C 14 & $5.729 \mathrm{kY}$ & 0.000123 & 8.15 \\
\hline NI 59 & $80 \mathrm{kY}$ & $1.8 E-05$ & 1.2 \\
\hline SN121m & 49.97 Years & $3.4 E-07$ & 0.02 \\
\hline ZR 93 & $1.500 \mathrm{MY}$ & $2.2 E-07$ & 0.01 \\
\hline $\mathrm{NB} 93 \mathrm{~m}$ & 13.60 Years & 2.1E-07 & 0.01 \\
\hline$\infty 060$ & 5.270 Yeare & $5.9 E-09$ & $3.9 E-0$ \\
\hline MO 93 & $3.498 \mathrm{kY}$ & $1.4 E-09$ & 9.1E-05 \\
\hline BE 10 & $1.600 \mathrm{MY}$ & $2.7 E-10$ & $1.8 E-05$ \\
\hline TC 99 & $213 \mathrm{kY}$ & $6.5 E-11$ & 4.3E-06 \\
\hline Y90 & 2.667 Days & 2.3E-11 & $1.5 E-06$ \\
\hline SR 90 & 29.12 Years & $2.3 E-11$ & $1.5 E-06$ \\
\hline AG108m & 127 Years & $4.2 E-12$ & 2.8E-07 \\
\hline NB 94 & $20.3 \mathrm{kY}$ & $2.3 E-12$ & 1.5E-07 \\
\hline TA182 & 115 Dayః & $5.3 E-13$ & $3.5 E-08$ \\
\hline HFt82 & $9 \mathrm{Mr}$ & $5.3 E-13$ & $3.5 E-08$ \\
\hline Si 32 & 650 Years & $4.6 E-13$ & $3.0 E-08$ \\
\hline P 32 & 14.3 Days & $4.6 E-13$ & $3.0 E-08$ \\
\hline AG108 & 2.37 Min. & $3.8 E-13$ & $2.5 E-08$ \\
\hline FE 55 & 2.6 Years & $9.2 E-14$ & $6.1 E-0$ \\
\hline RE187 & $50 \mathrm{GY}$ & $8.8 E-14$ & 5.8E-09 \\
\hline PB205 & $30 \mathrm{Mr}$ & $8.0 E-14$ & 5.3E-09 \\
\hline SB125 & 2.77 Years & $3.6 E-14$ & $2.4 E-09$ \\
\hline TE125m & 58 Days & $8.9 E-15$ & $5.9 E-10$ \\
\hline LU176 & $30 \mathrm{GY}$ & $1.2 E-17$ & $7.8 E-13$ \\
\hline TC 98 & $4.199 \mathrm{MY}$ & $1.3 E-18$ & $8.6 E-1$ \\
\hline$\vee 50$ & $4 E+07 \mathrm{GY}$ & $1.7 E-19$ & $1.1 E-1$ \\
\hline PB204 & $1 E+08 \mathrm{GY}$ & $1.3 E-19$ & $8.6 E-1$ \\
\hline IN115 & $5 E+05 \mathrm{GY}$ & $1.2 E-19$ & $8.2 \mathrm{E}-1$ \\
\hline $\mathrm{B} \mid 208$ & $368 \mathrm{kY}$ & $4.7 E-20$ & $3.1 E-1$ \\
\hline $\mathrm{Bl} 210 \mathrm{~m}$ & $3 M Y$ & $3.1 E-20$ & $2.1 E-1$ \\
\hline TL206 & $4.19 \mathrm{Min}$. & $3.1 E-20$ & $2.1 E-1$ \\
\hline PD107 & $6.496 \mathrm{MY}$ & 7.1E-21 & $4.7 \mathrm{E}-1$ \\
\hline TE123 & $10001 \mathrm{GY}$ & $5.3 E-21$ & $3.6 E-1$ \\
\hline 1R192 & 74.02 Days & $5.1 E-21$ & $3.4 E-1$ \\
\hline IA192m & 241 Years & $5.0 E-21$ & 3.3E-1 \\
\hline PT193 & 500 Years & $4.0 E-22$ & $2.6 E-1$ \\
\hline PO210 & 138.4 Days & $1.2 E-22$ & $8.3 E-1$ \\
\hline 1129 & $15.7 \mathrm{MY}$ & $1.2 E-25$ & $7.6 E-2$ \\
\hline AR 42 & 33 Years & $6.7 E-26$ & 4.5E-2 \\
\hline K42 & 12.36 Hours & $6.7 E-26$ & $4.5 E-2$ \\
\hline RH102 & 2.9 Yoars & $8.7 E-29$ & $5.8 \mathrm{E}$ \\
\hline AG109m & 39.6 Sec. & $1.0 E-29$ & 6.6 \\
\hline cotos & 1.27 Years & $1.0 E-29$ & 6.6 \\
\hline$B 10$ & 0 Stable & 0 & \\
\hline B 11 & O Stable & 0 & \\
\hline 812 & 0.02 Sec. & 0 & \\
\hline C 12 & O Stable & 0 & \\
\hline C 13 & O Stable & 0 & \\
\hline C 15 & 2.449 Sec. & 0 & \\
\hline F 19 & O Stable & 0 & \\
\hline F 20 & 11.4 Sec. & 0 & \\
\hline H 1 & O Stable & 0 & \\
\hline$H_{2}$ & 0 Stable & 0 & \\
\hline H 4 & 0.001 Sec. & 0 & \\
\hline 1125 & 59.7 Days & 0 & \\
\hline 1126 & 13.02 Days & 0 & \\
\hline 1127 & O Stable & 0 & \\
\hline 1128 & 24.98 Min. & 0 & \\
\hline 1130 & 12.36 Hours & 0 & \\
\hline $1130 \mathrm{~m}$ & 9 Min. & 0 & \\
\hline 1131 & 8.041 Days & 0 & \\
\hline 1132 & 2.3 Hours & 0. & \\
\hline K39 & O Stable & 0 & \\
\hline$K 40$ & $1.280 \mathrm{GY}$ & 0 & \\
\hline K41 & O Stable & 0 & \\
\hline$K 43$ & 22.6 Hours & 0 & \\
\hline K44 & 22 Min. & 0 & \\
\hline N 13 & 9.97 Min. & 0 & \\
\hline
\end{tabular}


Table 2.2. (contd)

Table 2-G. Decay Time $=300$ Years

\begin{tabular}{|c|c|c|c|}
\hline sotopo & Hatt-Lite & Cunies/kgl & Per Cont \\
\hline N1 63 & 92 Years & 0.000239 & 63.19 \\
\hline $\begin{array}{l}\text { C } 14 \\
\text { NI } 59\end{array}$ & $\begin{array}{r}5.729 \mathrm{kY} \\
80 \mathrm{kY}\end{array}$ & $\begin{array}{l}0.000 \\
0.0000\end{array}$ & $\begin{array}{r}31.83 \\
4.86\end{array}$ \\
\hline ZA 93 & $1.530 \mathrm{Mr}$ & $2.2 E-07$ & 0.058 \\
\hline $\mathrm{NB} 93 \mathrm{~m}$ & 13.60 Years & $2.1 E-07$ & 0.055 \\
\hline SN121m & 49.97 Years & $2.1 E-08$ & $5.6 E-03$ \\
\hline & 12.35 Years & $3.8 E-09$ & $1.0 E-03$ \\
\hline & 3.498 & $1.3 E-09$ & $3.5 E-04$ \\
\hline BE to & $1.600 \mathrm{MY}$ & $2.7 E-10$ & $7.2 E-05$ \\
\hline TC 99 & $213 \mathrm{kY}$ & $6.5 E-11$ & $1.7 E-05$ \\
\hline NB 94 & $20.3 \mathrm{kY}$ & $2.2 E-12$ & $5.9 E-07$ \\
\hline $38 m$ & 127 Years & $1.4 E-12$ & $3.8 E-07$ \\
\hline & 115 Days & $5.3 E-13$ & $1.4 E-07$ \\
\hline & & 5.3E-13 & $1.4 E-07$ \\
\hline & 14.3 & $3.7 E-13$ & $9.8 E-08$ \\
\hline S1 32 & 650 Years & $3.7 E-13$ & $9.8 E-08$ \\
\hline Y90 & 2.667 Days & $2.0 E-13$ & $5.2 E-\infty 8$ \\
\hline SR 90 & 29.12 Years & $2.0 E-13$ & $5.2 E-08$ \\
\hline 108 & 2.37 Min. & $1.3 E-13$ & $3.3 E-08$ \\
\hline 87 & 50 & $8.8 E-14$ & $2.3 E-08$ \\
\hline 205 & 30 & $8.0 E-14$ & 2.1E-08 \\
\hline LU176 & 30 & $1.2 E-17$ & $3.1 E-12$ \\
\hline TC 98 & $4.199 \mathrm{MY}$ & $1.3 E-18$ & $3.4 E-13$ \\
\hline V 50 & $4 E+07$ & $1.7 E-19$ & $4.5 E-14$ \\
\hline PB204 & $1 E+08$ & $1.3 E-19$ & $3.4 \mathrm{E}-14$ \\
\hline IN115 & $5 E+05$ GY & $1.2 E-19$ & $3.3 E-14$ \\
\hline B1208 & $368 \mathrm{kY}$ & 4.7E-20 & $1.3 E-14$ \\
\hline Bl210m & 3 & $3.1 E-20$ & $8.3 E-15$ \\
\hline TL206 & 4.19 & $3.1 E-20$ & $8.2 E-15$ \\
\hline$c$ & 5.27 & $2.2 E-20$ & $5.8 E-15$ \\
\hline 107 & $6.496 \mathrm{Mr}$ & $7.1 E-21$ & $1.9 E-15$ \\
\hline TE123 & $10001 \mathrm{GY}$ & $5.3 E-21$ & $1.4 E-15$ \\
\hline IR192 & 74.02 Days & $2.8 E-21$ & $7.5 E-16$ \\
\hline IR192m & 241 Years & $2.8 E-21$ & $7.4 \mathrm{E}-16$ \\
\hline PT193 & 500 Years & $2.9 E-22$ & $7.6 E-17$ \\
\hline PO210 & 138.4 Days & $1.2 \mathrm{E}-22$ & $3.3 E-17$ \\
\hline 1129 & $15.7 \mathrm{Mr}$ & $1.2 E-25$ & $3.0 E-20$ \\
\hline K 42 & 12.36 Hours & $6.7 E-26$ & $1.8 E-20$ \\
\hline AR 42 & 33 Years & $6.7 E-26$ & $1.8 E-20$ \\
\hline BA141 & 18.27 Min. & 0 & \\
\hline LA137 & $59.99 \mathrm{kY}$ & 0 & \\
\hline BA137m & $2.552 \mathrm{Mln}$. & 0 & \\
\hline LA139 & o Stable & 0 & \\
\hline LA140 & 1.676 Days & 0 & \\
\hline LA138 & $135 \mathrm{GY}$ & 0 & \\
\hline BA140 & 12.79 Days & 0 & \\
\hline$\Delta 141$ & 3.931 Hours & 0 & \\
\hline 1150 & O Stable & 0 & \\
\hline A134 & o Stable & 0 & \\
\hline 138 & 32.2 Min. & 0 & \\
\hline 130 & 0 Stable & 0 & \\
\hline 131 & 11.81 Days & 0 & \\
\hline BA131m & 15 Min. & 0 & \\
\hline 132 & de & 0 & \\
\hline 133 & 10.74 Years & 0 & \\
\hline SM151 & 89.99 & 0 & \\
\hline BA1 & 1.378 Hours & 0 & \\
\hline $\mathrm{BA} 133 \mathrm{~m}$ & 1.62 & 0 & \\
\hline SM148 & $8 E+06$ & 0 & \\
\hline SM149 & $1 E+07 \mathrm{GY}$ & 0 & \\
\hline BA135 & table & 0 & \\
\hline $135 \mathrm{~m}$ & 1.196 Days & 0 & \\
\hline BA136 & o Stable & 0 & \\
\hline $\mathrm{BA136m}$ & 0.308 Sec. & 0 & \\
\hline BA137 & - Stable & 0 & \\
\hline BAISO & ablo & 0 & \\
\hline SM147 & $107 \mathrm{GY}$ & 0 & \\
\hline CE139 & 137.6 Days & 0 & \\
\hline & 17.7 & 0 & \\
\hline PM147 & 2.623 Years & 0 & \\
\hline
\end{tabular}

Table 2-H. Decay Time $=1000$ Years

\begin{tabular}{|c|c|c|c|}
\hline otope & Hati-Lito & Curies/kgu & Per Cent \\
\hline $\begin{array}{l}C 14 \\
\text { VI } 59\end{array}$ & $5.729 \mathrm{kY}$ & 0.000111 & 84.74 \\
\hline $\begin{array}{l}\text { NI59 } \\
\text { NI } 63\end{array}$ & $\begin{array}{l}80 \mathrm{kY} \\
92 \mathrm{Yor}\end{array}$ & $\begin{array}{l}0.000018 \\
1.2 E-06\end{array}$ & $\begin{array}{l}14.00 \\
0.939\end{array}$ \\
\hline ZR 93 & $1.530 \mathrm{MY}$ & $2.2 E-07$ & 0.168 \\
\hline NB 93m & 13.60 Yoars & $2.1 E-07$ & 0.159 \\
\hline $\begin{array}{l}\text { MO } 93 \\
\text { BE } 10\end{array}$ & $3.498 \mathrm{kY}$ & $1.2 E-09$ & $8.8 E-04$ \\
\hline BE 10 & $1.600 \mathrm{MY}$ & $2.7 E-10$ & $2.1 E-04$ \\
\hline $\begin{array}{l}\text { TC } 99 \\
\text { NB } 94\end{array}$ & $\begin{array}{l}213 \mathrm{kY} \\
20.3 \mathrm{kY}\end{array}$ & $\begin{array}{l}6.5 \mathrm{E}-11 \\
2.2 \mathrm{E}-12\end{array}$ & $\begin{array}{l}5.0 E-05 \\
17 E-06\end{array}$ \\
\hline SN121m & 49.97 Yoars & $1.3 E-12$ & $\begin{array}{l}1.7 E-06 \\
9.8 E-07\end{array}$ \\
\hline TA182 & 115 Days & $5.3 E-13$ & $4.1 E-07$ \\
\hline HF182 & $9 \mathrm{MY}$ & $5.3 E-13$ & $4.1 E-07$ \\
\hline $\begin{array}{l}\text { P } 32 \\
S 132\end{array}$ & 14.3 Days & $\begin{array}{l}1.7 E-13 \\
17 E-13\end{array}$ & $\begin{array}{l}1.3 E-07 \\
1.3 E-07\end{array}$ \\
\hline RE187 & $50 \mathrm{GY}$ & $8.8 E-14$ & $6.7 E-08$ \\
\hline $\begin{array}{l}\text { PB205 } \\
\text { AG106r }\end{array}$ & $\begin{array}{l}30 \mathrm{Mr} \\
127 \text { Yoars }\end{array}$ & $\begin{array}{l}8.0 E-14 \\
3.1 E-14\end{array}$ & $\begin{array}{l}6.2 E-08 \\
2.4 E-08\end{array}$ \\
\hline AG108 & 2.37 Min. & $\begin{array}{l}3.15-14 \\
2.8 E-15\end{array}$ & $\begin{array}{l}2.4 E-08 \\
2.1 E-09\end{array}$ \\
\hline LU17 & $30 \mathrm{C}$ & $1.2 E-17$ & $9.0 E-12$ \\
\hline TC 98 & $4.199 \mathrm{MN}$ & $1.3 E-18$ & $1.0 E-12$ \\
\hline & $4 E+0$ & $1.7 E-19$ & $1.3 E-13$ \\
\hline PB204 & $1 E+\infty \mathrm{GY}$ & $1.3 E-19$ & $1.0 E-13$ \\
\hline IN115 & $5 E+05 \mathrm{GY}$ & $1.2 \mathrm{E}-19$ & $9.5 E-14$ \\
\hline Bl208 & $368 \mathrm{kY}$ & $4.7 E-20$ & $3.6 E-14$ \\
\hline $\mathrm{Bl} 210 \mathrm{~m}$ & $3 M Y$ & $3.1 E-20$ & $2.4 E-14$ \\
\hline TL206 & $4.19 \mathrm{Min}$. & $3.1 E-20$ & $2.4 E-14$ \\
\hline SR 90 & 29.12 Years & $1.1 E-20$ & $8.8 E-15$ \\
\hline$Y 90$ & 2.667 & $1.1 E-20$ & $8.8 E-15$ \\
\hline $\begin{array}{l}\text { PD107 } \\
\text { TE123 }\end{array}$ & $\begin{array}{l}6.496 \mathrm{MY} \\
10001 \mathrm{GY}\end{array}$ & $\begin{array}{l}7.1 E-21 \\
5.3 E-21\end{array}$ & $\begin{array}{l}5.5 \mathrm{E}-15 \\
4.1 \mathrm{E}-15\end{array}$ \\
\hline IR192 & 74.02 Days & $4.3 E-22$ & 3.3E-16 \\
\hline IA192m & 241 Yoars & $4.3 E-22$ & 3.3E-16 \\
\hline PT193 & 500 Years & $1.4 E-22$ & $1.1 E-16$ \\
\hline PO210 & 138.4 Days & $1.2 E-22$ & $9.6 E-17$ \\
\hline 1129 & $15.7 \mathrm{Mr}$ & $1.2 E-25$ & $8.8 E-20$ \\
\hline H 3 & 12.35 Years & $3.3 E-26$ & $2.5 E-20$ \\
\hline $\begin{array}{l}\text { AR 42 } \\
\mathrm{K} 42\end{array}$ & 33 Years & $2.8 E-32$ & $2.1 \mathrm{E}-26$ \\
\hline $\begin{array}{l}\text { n } 42 \\
\text { LA141 }\end{array}$ & $\begin{array}{l}\text { 12.36 Hours } \\
3.931 \text { Hours }\end{array}$ & & $E-26$ \\
\hline LA137 & $\begin{array}{l}59.99 \mathrm{kY} \\
\end{array}$ & & \\
\hline CS137 & 30 Yoars & 0 & \\
\hline BA141 & 18.27 Min. & & \\
\hline BA131 & 11.81 Days & 0 & \\
\hline $\operatorname{Cs} 138$ & 32.2 Min. & 0 & \\
\hline SM148 & $8 E+06 \mathrm{GY}$ & & \\
\hline LA139 & O Stable & 0 & \\
\hline LA140 & 1.676 Days & 0 & \\
\hline LA138 & $135 \mathrm{GY}$ & & \\
\hline BA133 & 10.74 Yoars & 0 & \\
\hline $\begin{array}{l}\text { BA140 } \\
\text { BA135 }\end{array}$ & $\begin{array}{l}\text { 12.79 Days } \\
\text { O Stablo }\end{array}$ & 0 & \\
\hline $\begin{array}{l}\text { BA135 } \\
\text { BA136 }\end{array}$ & O Stablo & 0 & \\
\hline SM151 & 89.99 Yoars & ? & \\
\hline BA133m & $1.62 \mathrm{Da}$ & 0 & \\
\hline SM15O & o st & & \\
\hline BA132 & $0 \mathrm{St}$ & 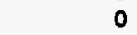 & \\
\hline BA134 & 0 Stable & 0 & \\
\hline BA130 & 0 St & & \\
\hline BA135n & $1.196 \mathrm{D}$ & & \\
\hline BA139 & 1.378 Hours & & \\
\hline BA131m & $15 \mathrm{M}$ & & \\
\hline BA137 & & & \\
\hline Ra137m & (2) & & \\
\hline & & & \\
\hline BA138 & 0 Stable & 0 & \\
\hline BA136m & $0.308 \mathrm{~S}$ & & \\
\hline SM1 & 340 Days & 0 & \\
\hline CE13 & 0 Stable & 0 & \\
\hline & $2.623 \mathrm{Yc}$ & 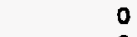 & \\
\hline PM148 & 5.37 Days & 0 & \\
\hline
\end{tabular}


Table 2.2. (contd)

Table 2-I. Decay Time $=3000$ Yoars

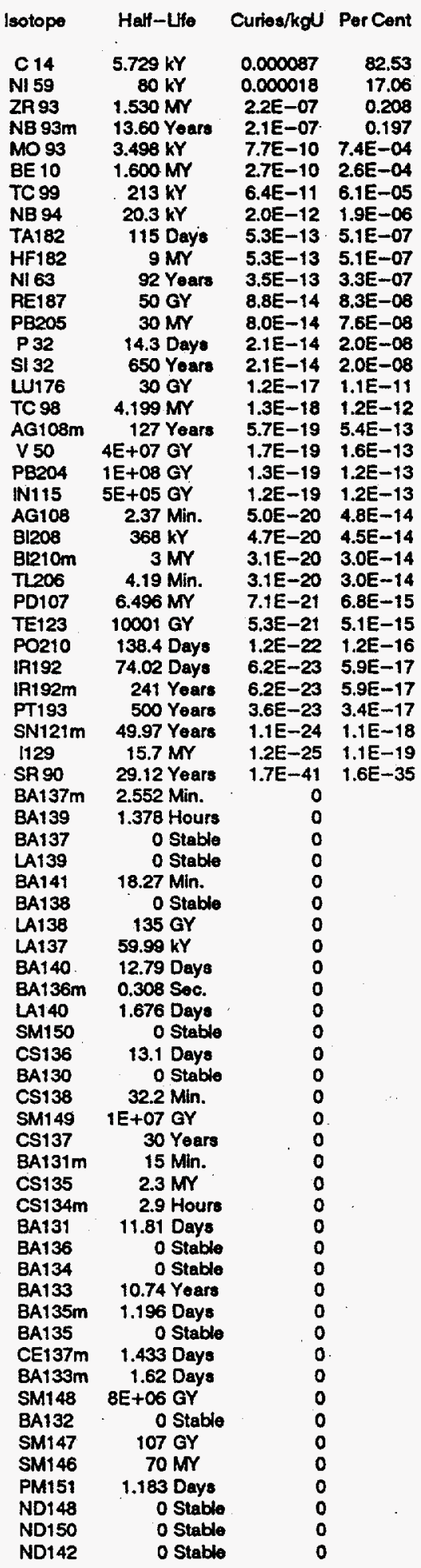


Table 2.3. Radionuclide Content of Actinides in Hanford Reactor Fuel for Various Decay Times

Table 3-A. Decay Time = 9 Yoar

\begin{tabular}{|c|c|c|c|}
\hline Isotope & Hat-Lito & Curios/kgU & Per Cont \\
\hline PU241 & 14.4 Years & 0.4798 & 88.12 \\
\hline PU239 & $24.06 \mathrm{KY}$ & 0.0489 & 8.98 \\
\hline PU240 & $6.537 \mathrm{kY}$ & 0.0105 & 1.92 \\
\hline PU238 & 87.74 Years & 0.00270 & 0.496 \\
\hline AM241 & 432.2 Yoars & 0.00083 & 0.152 \\
\hline $\mathrm{CN} 242$ & 163.2 Days & 0.00036 & 0.067 \\
\hline U234 & $244.5 \mathrm{kY}$ & 0.00035 & 0.064 \\
\hline PA234m & 1.17 Min. & 0.00033 & 0.061 \\
\hline TH234 & 24.1 Days & 0.00033 & 0.061 . \\
\hline U238 & $4.468 \mathrm{GY}$ & 0.00033 & 0.061 \\
\hline CM244 & 18.11 Years & $1.5 E-05$ & $2.8 E-03$ \\
\hline TH231 & 1.063 Days & $1.4 E-0.5$ & $2.6 E-03$ \\
\hline U235 & $703.8 \mathrm{Mr}$ & $1.4 E-05$ & $2.6 E-03$ \\
\hline U237 & 6.75 Days & $1.2 E-05$ & 2.2E-03 \\
\hline U236 & $23.41 \mathrm{MY}$ & $8.6 E-06$ & $1.6 E-03$ \\
\hline NP237 & $2.14 \mathrm{MY}$ & $5.2 E-\infty 6$ & $9.5 E-04$ \\
\hline PA233 & 27 Days & $5.2 E-\infty 6$ & $9.5 E-04$ \\
\hline AM242m & 152 Years & $6.9 E-07$ & $1.3 E-04$ \\
\hline AM242 & 16.02 Hours & $6.9 \bar{E}-07$ & $1.3 E-04$ \\
\hline PU236 & 2.851 Years & $5.4 E-07$ & $9.8 E-05$ \\
\hline PU242 & $386.9 \mathrm{kY}$ & $4.6 E-07$ & $8.5 E-05$ \\
\hline PA234 & 6.7 Hours & $4.3 E-07$ & $8.0 E-05$ \\
\hline$A M 243$ & $7.38 \mathrm{kY}$ & $1.6 E-07$ & $2.9 E-05$ \\
\hline NP239 & 2.355 Days & $1.6 E-07$ & 2.9E-05 \\
\hline CM243 & 28.5 Years & $3.0 E-08$ & $5.5 E-06$ \\
\hline U232 & 72 Years & $2.7 E-08$ & $5.0 E-05$ \\
\hline PU237 & 45.6 Days & $1.2 E-08$ & $2.2 E-05$ \\
\hline PO216 & 0.15 Sec. & $8,1 E-09$ & $1.5 E-05$ \\
\hline PB212 & 10.64 Hours & $8.1 E-09$ & $1.5 E-0.5$ \\
\hline RA224 & 3.66 Days & 8.1E-09 & $1.5 E-06$ \\
\hline RN220 & $55.6 \mathrm{Sec}$. & $8.1 E-09$ & $1.5 E-06$ \\
\hline $\mathrm{B} 1212$ & 1.009 Hours & $8.1 E-09$ & $1.5 E-06$ \\
\hline $\mathrm{TH} 228$ & 1.913 Years & $8.1 E-09$ & $1.5 E-06$ \\
\hline NP235 & 1.084 Years & 6.7E-09 & $1.2 E-06$ \\
\hline PO212 & 3E-07 Sec. & $5.2 \mathrm{E}-09$ & $9.6 E-07$ \\
\hline TH230 & $\pi \mathrm{kY}$ & $3.8 E-09$ & $7.0 E-07$ \\
\hline NP238 & 2.117 Days & $3.5 E-09$ & $6.4 E-07$ \\
\hline TL208 & $3.07 \mathrm{Min}$ & $2.9 E-09$. & $5.4 E-07$ \\
\hline U233 & $158.5 \mathrm{kY}$ & $3.9 E-10$ & $7.2 E-08$ \\
\hline PA231 & $32.77 \mathrm{kr}$ & $3.9 E-10$ & $7.2 E-08$ \\
\hline $\mathrm{CM} 245$ & $8.499 \mathrm{kY}$ & $8.3 E-11$ & $1.5 E-08$ \\
\hline NP236 & $115 \mathrm{kY}$ & $1.1 E-11$ & $2.1 E-09$ \\
\hline PB211 & 36.1 Min. & $7.8 E-12$ & $1.4 E-09$ \\
\hline RN219 & $3.96 \mathrm{Sec}$. & $7.8 E-12$ & $1.4 E-09$ \\
\hline PO215 & 0.002 Sec. & $7.8 E-12$ & $1.4 E-09$ \\
\hline RAZ23 & 11.43 Days & $7.8 E-12$ & $1.4 E-09$ \\
\hline$B 1211$ & 2.13 Min. & $7.8 E-12$ & $1.4 E-09$ \\
\hline$A C 227$ & 21.77 Years & $7.8 E-12$ & $1.4 E-09$ \\
\hline TL207 & 4.T7 Min. & $7.8 E-12$ & $1.4 E-09$ \\
\hline tinize & 18.72 Days & $7.7 \mathrm{E}-12$ & $1.4 E-09$ \\
\hline PO218 & $3.05 \mathrm{Min}$. & $1.0 E-12$ & $1.8 E-10$ \\
\hline RN2202 & 3.824 Days & $1.0 E-12$ & $1.8 E-10$ \\
\hline RA226 & $1.6 \mathrm{kY}$ & $1.0 E-12$ & $1.8 E-10$ \\
\hline PB214 & 26.8 Min. & $1.0 E-12$ & $1.8 E-10$ \\
\hline B1214 & 19.9 Min. & $1.0 E-12$ & $1.8 E-10$ \\
\hline PO214 & 2E-04 Sec. & $1.0 E-12$ & $1.8 E-10$ \\
\hline CM246 & $4.731 \mathrm{kY}$ & $6.9 E-13$ & $1.3 E-10$ \\
\hline FF:23 & $21.8 \mathrm{Min}$. & $1.1 E-13$ & $2.0 E-11$ \\
\hline TH229 & $7.339 \mathrm{kY}$ & $6.4 E-14$ & $1.2 E-11$ \\
\hline RA225 & 14.8 Days & $6.4 E-14$ & $1.2 E-11$ \\
\hline FPO21 & 4.8 Min. & $6.4 E-14$ & $1.2 E-11$ \\
\hline$A C 225$ & 10 Days & $6.4 E-14$ & $1.2 E-11$ \\
\hline 81213 & 45.65 Min. & $6.4 E-14$ & $1.2 E-11$ \\
\hline AT 217 & 0.032 Sec. & $6.4 E-14$ & $1.2 E-11$ \\
\hline P8209 & 3.3 Hours & $6.4 E-14$ & $1.2 \mathrm{E}-11$ \\
\hline $\mathrm{PO} 213$ & 4E-06 Soc. & $6.2 E-14$ & $1.1 E-11$ \\
\hline CM241 & 36 Days & $5.9 E-14$ & $1.1 E-11$ \\
\hline PO211 & 0.56 Sec. & $2.2 E-14$ & $4.0 E-12$ \\
\hline PB210 & 22.3 Years & $1.3 E-14$ & $2.3 E-12$ \\
\hline$B 1210$ & 5.012 Days & $1.3 E-14$ & $2.3 E-12$ \\
\hline
\end{tabular}

Table 3-B. Decay Time $=3$ Yoars

\begin{tabular}{|c|c|c|c|}
\hline lootope & Haf-LHo & Curies/kgu & Por Cont \\
\hline PU241 & 14.4 Yoars & 0.4357 & 86.89 \\
\hline PU239 & $24.06 \mathrm{kY}$ & 0.0489 & 9.75 \\
\hline PU240 & $6.537 \mathrm{kY}$ & 0.0105 & 2.08 \\
\hline PU238 & 87.74 Years & 0.00266 & 0.530 \\
\hline AM241 & 432.2 Years & 0.00229 & 0.457 \\
\hline U234 & $244.5 \mathrm{kY}$ & 0.00035 & 0.070 \\
\hline U238 & $4.468 \mathrm{GY}$ & 0.00033 & 0.067 \\
\hline TH234 & 24.1 Days & 0.00033 & 0.067 \\
\hline PA234m & $1.17 \mathrm{Min}$. & 0.00033 & 0.067 \\
\hline CM242 & 163.2 Days & $1.7 E-05$ & $3.4 E-03$ \\
\hline CM1244 & 18.11 Years & $1.4 E-05$ & $2.8 E-03$ \\
\hline U235 & $703.8 \mathrm{MY}$ & $1.4 E-05$ & $2.8 E-03$ \\
\hline$\pi+231$ & 1.063 Daye & $1.4 E-05$ & $2.8 E-03$ \\
\hline U237 & 6.75 Days & 1.1E-05 & 2.1E-03 \\
\hline บ236 & $23.41 \mathrm{MY}$ & $8.6 E-\infty 6$ & $1.7 E-03$ \\
\hline NP237 & $2.14 \mathrm{Mr}$ & $5.2 E-06$ & $1.0 E-03$ \\
\hline PA233 & 27 Days & $5.2 E-06$ & $1.0 E-03$ \\
\hline $\mathrm{AM} 242 \mathrm{~m}$ & 152 Years & $6.9 E-07$ & $1.4 E-04$ \\
\hline AM242 & 16.02 Hours & $6.8 E-07$ & $1.4 E-04$ \\
\hline PU242 & $386.9 \mathrm{kY}$ & $4.6 E-07$ & $9.3 E-05$ \\
\hline PA234 & 6.7 Hours & $4.3 E-07$ & $8.6 E-05$ \\
\hline PU236 & 2.851 Years & $3.3 E-07$ & $6.6 E-05$ \\
\hline$A M 243$ & $7.38 \mathrm{kY}$ & $1.6 E-07$ & 3.1E-05 \\
\hline NP239 & 2.355 Days & $1.6 E-07$ & $3.1 E-05$ \\
\hline U232 & 72 Years & $3.5 E-08$ & $7.0 E-06$ \\
\hline $\mathrm{CM} 243$ & 28.5 Years & $2.9 E-08$ & $5.7 E-06$ \\
\hline RNZ2O & 55.6 Sec. & $2.0 E-08$ & $4.1 E-06$ \\
\hline PB212 & 10.64 Hours & $2.0 E-0 B$ & $4.1 E-06$ \\
\hline $\operatorname{RAZ24}$ & 3.65 Deys & $2.0 E-08$ & $4.1 E-05$ \\
\hline PO216 & $0.15 \mathrm{Sec}$ & $2.0 E-08$ & $4.1 E-06$ \\
\hline $8 ! 212$ & 1.009 Hours & $2.0 E-08$ & 4.1E-06 \\
\hline$\pi+228$ & 1.913 Years & $2.0 E-08$ & $4.1 E-\infty$ \\
\hline $\mathrm{PO} 212$ & $3 E-07$ Sec. & $1.3 E-08$ & $2.6 \bar{E}-06$ \\
\hline$T H 230$ & $77 \mathrm{kY}$ & $1.0 E-08$ & $2.0 E-06$ \\
\hline TL208 & $3.07 \mathrm{Min}$. & 7.3E-09 & $1.5 E-06$ \\
\hline NP238 & 2.117 Days & $3.4 E-09$ & $6.9 E-07$ \\
\hline NP235 & 1.084 Years & $1.9 E-09$ & $3.7 E-07$ \\
\hline PA231 & $32.77 \mathrm{kY}$ & $9.9 E-10$ & $2.0 E-07$ \\
\hline U233 & $158.5 \mathrm{kY}$ & $4.4 E-10$ & $8.8 E-08$ \\
\hline CM245 & $8.499 \mathrm{kY}$ & $8.3 E-11$ & $1.7 E-08$ \\
\hline PN219 & 3,96 Sec. & $5.0 E-11$ & $1.0 E-08$ \\
\hline PO215 & 0.002 Sec. & $5.0 E-11$ & $1.0 E-08$ \\
\hline PB211 & 36.1 Min. & $5.0 E-11$ & $1.0 E-08$ \\
\hline 81211 & $2.13 \mathrm{Min}$. & 5.OE- 11 & 1.OE-OB \\
\hline RA223 & 11.43 Days & $5.0 E-11$ & $1.0 E-08$ \\
\hline$A C 227$ & 21.77 Years & $5.0 E-11$ & $1.0 E-08$ \\
\hline TL207 & $4.77 \mathrm{Min}$. & $5.0 E-11$ & $1.0 E-08$ \\
\hline $7 \mathrm{H} 227$ & 18.72 Days & $5.0 E-11$ & $9.9 E-09$ \\
\hline NP236 & $115 \mathrm{kY}$ & $1.1 E-11$ & 2.3E-09 \\
\hline RA226 & $1.6 \mathrm{kY}$ & $7.0 E-12$ & $1.4 E-09$ \\
\hline RN222 & 3.824 Days & $7.0 E-12$ & $1.4 E-09$ \\
\hline PO218 & $3.05 \mathrm{Min}$. & $7.0 E-12$ & $1.4 E-09$ \\
\hline PB214 & 26.8 Min. & $7.0 E-12$ & $1.4 E-09$ \\
\hline $\mathrm{B} 1214$ & $19.9 \mathrm{Min}$. & $7.0 E-12$ & $1.4 E-09$ \\
\hline PO214 & 2E-04 Soc. & $7.0 E-12$ & $1.4 \bar{E}-09$ \\
\hline$F R 223$ & $21.8 \mathrm{Min}$. & $6.9 E-13$ & $1.4 \bar{E}-10$ \\
\hline CM246 & $4.731 \mathrm{kY}$ & $6.9 E-13$ & $1.4 E-10$ \\
\hline $\mathrm{B} 1210$ & 5.012 Days & $2.3 E-13$ & $4.5 E-11$ \\
\hline PB210 & 22.3 Yoars & $2.3 E-13$ & $4.5 E-11$ \\
\hline PU237 & 45.6 Days & $1.8 E-13$ & $3.6 E-11$ \\
\hline$A C 225$ & 10 Days & $1.4 E-13$ & $2.8 E-11$ \\
\hline AT217 & 0.032 Sec. & $1.4 E-13$ & $2.8 E-11$ \\
\hline P8209 & 3.3 Hours & $1.4 E-13$ & $2.8 E-11$ \\
\hline RA225 & 14.8 Days & $1.4 E-13$ & $2.8 E-11$ \\
\hline $7+229$ & $7.339 \mathrm{kY}$ & $1.4 E-13$ & $2.8 E-11$ \\
\hline FPO21 & 4.8 Min. & $1.4 E-13$ & $2.8 E-11$ \\
\hline $\mathrm{B} 1213$ & $45.65 \mathrm{Min}$. & $1.4 E-13$ & $2.8 E-11$ \\
\hline PO210 & 138.4 Days & $1.4 E-13$ & $2.8 E-11$ \\
\hline & 0.56 Sec. & $1,4 E-13$ & $2.8 E-11$ \\
\hline PO213 & E-06 Sec. & $1.4 E-13$ & $2.8 E-11$ \\
\hline
\end{tabular}


Table 2.3. (contd)

Tabie 3-C. Decay Time = 10 Yoars

\begin{tabular}{|c|c|c|c|}
\hline olope & Hatt-Lito & Curies/kgU & $\mathrm{Po}_{\mathrm{O}}$ \\
\hline $\begin{array}{l}\text { PU241 } \\
\text { PU239 }\end{array}$ & $\begin{array}{l}14.4 \text { Years } \\
24.06 \mathrm{kY}\end{array}$ & $\begin{array}{l}0.3111 \\
0.0489\end{array}$ & \\
\hline PU240 & $6.537 \mathrm{kY}$ & 0.0104 & \\
\hline AM241 & 432.2 Yonrs & 0.00639 & \\
\hline PU238 & 87.74 Yoata & 0.00251 & \\
\hline U234 & - & 0.00035 & \\
\hline U238 & $4.468 \mathrm{GY}$ & 0.00033 & \\
\hline PA234m & 1.17 Min. & 0.00033 & \\
\hline & 24.1 Days & 0.00033 & \\
\hline TH231 & 1.063 Days & $1.4 \mathrm{E}-05$ & 3.7E-0 \\
\hline U235 & 800.0010 & $1.4 E-05$ & $3.7 E-0$ \\
\hline CIN244 & 18.11 Years & 1.1E-05 & $2.9 E-0$ \\
\hline U236 & $23.41 \mathrm{MY}$ & $8.6 E-06$ & $2.3 E-0$ \\
\hline U237 & Days & $7.6 E-06$ & $2.0 E-03$ \\
\hline NP237 & 2.14 & $5.2 E-06$ & $1.4 E-03$ \\
\hline PA233 & 27 & & \\
\hline AM242m & 152 Years & $6.7 E-07$ & $1.8 E-0$ \\
\hline AM242 & ours & $6.6 \mathrm{E}-07$ & \\
\hline CM242 & 163.2 Days & $5.5 E-07$ & \\
\hline PU242 & $386.9 \mathrm{kY}$ & $4.6 E-07$ & $1.2 E-04$ \\
\hline PA234 & ours & $4.3 E-07$ & \\
\hline AM243 & 7.3 & $1.6 E-07$ & $4.1 E-05$ \\
\hline NP239 & ays & $1.6 E-07$ & 4.1E-05 \\
\hline PU236 & ars & $6.0 E-08$ & $1.6 E-0$ \\
\hline U232 & ars & $E-08$ & $1.1 E-05$ \\
\hline RA224 & 3.66 Deys & $.0 E-08$ & $1.1 E-05$ \\
\hline RN220 & 55. & 08 & $1.1 E-05$ \\
\hline PB212 & Hours & $.0 E-08$ & $1.1 E-05$ \\
\hline $\mathrm{B} / 212$ & Hours & $.0 E-08$ & $1.1 E-05$ \\
\hline PO216 & 0.15 Sec. & 88 & 1.1 \\
\hline TH228 & 1.91 & $E-08$ & $1.1 E$ \\
\hline $\mathrm{TH} 230$ & $77 \mathrm{kY}$ & $E-08$ & $8.4 E-06$ \\
\hline PO212 & $3 E-07$ Sec. & $6 E-08$ & $6.7 E-06$ \\
\hline CM243 & 28.5 Years & $4 E-08$ & $6.4 E-06$ \\
\hline TL208 & 3.07 Min. & 08 & $3.8 E-06$ \\
\hline NP238 & 2.11 & $E-09$ & $8.8 E-07$ \\
\hline PA231 & $32.77 \mathrm{kY}$ & IE-09 & $8.2 E-07$ \\
\hline U233 & $158.5 \mathrm{kY}$ & $.0 E-10$ & $1.6 E-07$ \\
\hline RA223 & 11.43 Days & $1.6 E-10$ & $1.2 E-07$ \\
\hline PO215 & 0.002 Sec. & $6 E-10$ & $1.2 E-07$ \\
\hline FN219 & Sec. & $6 E-10$ & $1.2 E-07$ \\
\hline PB211 & $36.1 \mathrm{Min}$. & $6 E-10$ & $1.2 \mathrm{E}-07$ \\
\hline $\mathrm{B} 1211$ & 2.13 Min. & $6 E-10$ & $1.2 E-07$ \\
\hline$A C 227$ & 21.77 Years & $6 E-10$ & $1.2 E-07$ \\
\hline TL207 & 4.77 Min. & $6 E-10$ & $1.2 E-07$ \\
\hline$\pi+227$ & 18.72 Days & $5 E-10$ & $1.2 E-07$ \\
\hline CM245 & $8.499 \mathrm{kY}$ & $3 E-11$ & $2.2 E-08$ \\
\hline RA226 & $1.6 \mathrm{kY}$ & $7.1 E-11$ & $1.9 E-08$ \\
\hline PO218 & $3.05 \mathrm{Min}$. & $7.1 E-11$ & $1.9 E-08$ \\
\hline RN222 & 3.824 Days & 1 & $1.9 E-08$ \\
\hline 81214 & $19.9 \mathrm{Min}$. & -11 & $1.9 E-08$ \\
\hline PE214 & 26.8 Min. & 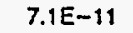 & $1.9 E-08$ \\
\hline $\mathrm{PO} 214$ & 2E-04 Sec. & $7.1 E-11$ & $1.9 E-08$ \\
\hline & 1.084 Years & 2.1E- & $5.5 E-09$ \\
\hline & $115 \mathrm{kY}$ & $1.1 E-11$ & $3.0 E-09$ \\
\hline & 5.012 Days & $6.9 E-12$ & $1.8 \mathrm{E}-09$ \\
\hline & T38.4 Days & $6.9 E-12$ & $1.8 E-09$ \\
\hline & 22.3 Years & $9 E-12$ & $1.8 E-09$ \\
\hline & $21.8 \mathrm{Min}$. & $6.3 E-12$ & $1.7 E-09$ \\
\hline & 0.56 Sec. & $1.3 E-12$ & $3.4 E-10$ \\
\hline & $4.731 \mathrm{kY}$ & $6.9 E-13$ & $1.8 E-10$ \\
\hline & 45.65 Min. & $4.9 E-13$ & $1.3 E-10$ \\
\hline & 0.032 Sec. & $4.9 E-13$ & $1.3 E-10$ \\
\hline & 14.8 Deys & $4.9 E-13$ & $1.3 E-10$ \\
\hline & $7.339 \mathrm{kY}^{\prime}$ & $4.9 E-13$ & $1.3 E-10$ \\
\hline & 3.3 Hours & $4.9 E-13$ & $1.3 E-10$ \\
\hline & 10 Days & $4.9 E-13$ & $1.3 E-10$ \\
\hline & 4.8 & $4.9 E-13$ & $1.3 E-10$ \\
\hline & $-\infty$ & -13 & $1.3 E-10$ \\
\hline & 2.2 & -14 & 2.86 \\
\hline
\end{tabular}

Table 3-D. Docay Time $=30$ Yoars

\begin{tabular}{|c|c|c|c|}
\hline Isolopo & Hatf-Uilo & Curies/kgU & Per Cent \\
\hline PU241 & 14.4 Yoara & 0.1188 & 61.20 \\
\hline PU239 & $24.06 k$ & 0.0489 & 25.17 \\
\hline AM241 & 432.2 Years & 0.0125 & 6.43 \\
\hline PU240 & $6.537 \mathrm{kY}$ & 0.0104 & 5.37 \\
\hline PU238 & 87.74 Yeare & 0.00215 & 1.11 \\
\hline U234 & $244.5 \mathrm{kY}$ & 0.00035 & 0.180 \\
\hline PA234m & $1.17 \mathrm{Min}$. & 0.00033 & 0.172 \\
\hline U238 & $4.468 \mathrm{GY}$ & 0.00033 & 0.172 \\
\hline TH234 & 24.1 Days & 0.00033 & 0.172 \\
\hline U235 & $703.8 \mathrm{Mr}$ & $1.4 E-0.5$ & $7.3 E-03$ \\
\hline TH231 & 1.063 Days & $1.4 E-05$ & $7.3 E-03$ \\
\hline U236 & $23.41 \mathrm{MY}$ & $8.6 E-06$ & $4.4 E-03$ \\
\hline NP237 & $2.14 M Y$ & $5.3 E-06$ & $2.7 E-03$ \\
\hline PA233 & 27 Days & $5.3 E-06$ & $2.7 E-03$ \\
\hline $\mathrm{CM} 244$ & 18.11 Years & $5.1 E-\infty 6$ & $2.6 E-03$ \\
\hline บ237 & 6.75 Days & $2.9 E-06$ & $1.5 E-03$ \\
\hline AM242m & 152 Years & $6.1 E-07$ & $3.1 E-04$ \\
\hline AM242 & 16.02 Hours & $6.1 E-07$ & $3.1 E-04$ \\
\hline CM242 & 163.2 Days & $5.0 E-07$ & $2.6 \mathrm{E}-04$ \\
\hline PU242 & $386.9 \mathrm{kY}$ & $4.6 E-07$ & $2.4 E-04$ \\
\hline PA234 & 6.7 Hours & $4.3 E-07$ & $2.2 E-04$ \\
\hline NP239 & 2.355 Days & $1.6 E-07$ & 8.1E-05 \\
\hline AM243 & $7.38 \mathrm{kY}$ & $1.6 E-07$ & 8.1E-05 \\
\hline TH 230 & $77 \mathrm{kY}$ & $9.5 E-08$ & $4.9 E-05$ \\
\hline PB212 & 10.64 Hours & $3.8 E-08$ & $2.0 E-05$ \\
\hline RN220 & 55.6 Soc. & $3.8 E-08$ & 2.0E-05 \\
\hline RA224 & 3.66 Days & $3.8 E-08$ & 2.0E-05 \\
\hline PO216 & 0.15 Sec. & $3.8 E-08$ & $2.0 E-05$ \\
\hline 81212 & 1.009 Hours & $3.8 E-08$ & $2.0 E-05$ \\
\hline$T+228$ & 1.913 Years & $3.8 E-08$ & $2.0 \mathrm{E}-05$ \\
\hline U232 & ears & $1.7 E-08$ & $1.9 E-05$ \\
\hline $\mathrm{PO}_{212}$ & 3E-07 Sec. & $2.5 E-08$ & $1.3 E-05$ \\
\hline $\mathrm{CM} 243$ & 28.5 Years & $1.5 E-08$ & $7.7 E-06$ \\
\hline TL208 & 3.07 Min. & $1.4 E-08$ & $7.1 E-06$ \\
\hline PA231 & $32.77 \mathrm{kY}$ & $9.1 E-09$ & $4.7 E-06$ \\
\hline BI211 & $2.13 \mathrm{Min}$. & $3.3 E-09$ & $1.7 E-06$ \\
\hline RA223 & 11.43 Days & 3.3E-09 & $1.7 E-06$ \\
\hline RN219 & $3.96 \mathrm{Sec}$. & 3.3E-09 & $1.7 E-06$ \\
\hline P8211 & 36.1 Min. & $3.3 E-09$ & 1.7E-06 \\
\hline PO215 & 0.002 Sec. & $3.3 E-09$ & $1.7 E-06$ \\
\hline$A C 227$ & 21.77 Years & $3.3 E-09$ & $1.7 E-06$ \\
\hline TL207 & 4.77 Min. & $E-09$ & $1.7 E-06$ \\
\hline TH227 & 18.72 & $.2 E-09$ & $1.7 E-06$ \\
\hline NP238 & 2.117 Days & DE-OS & $1.6 E-06$ \\
\hline U233 & $158.5 \mathrm{kY}$ & $1 E-09$ & $5.4 E-07$ \\
\hline PO218 & $3.05 \mathrm{Min}$. & $2 E-10$ & $3.2 E-07$ \\
\hline RN222 & 3.824 Days & $6.2 E-10$ & $3.2 E-07$ \\
\hline RA226 & $1.6 \mathrm{kY}$ & $6.2 \mathrm{E}-10$ & $3.2 E-07$ \\
\hline PB214 & $26.8 \mathrm{Min}$. & $6.2 E-10$ & $3.2 E-07$ \\
\hline $\mathrm{B} 1214$ & 19.9 Min. & $6.2 E-10$ & 3.2E-07 \\
\hline PO214 & $2 E-04$ Sec. & $E-10$ & $3.2 E-07$ \\
\hline PU236 & 2.851 Years & $E-10$ & $2.4 E-07$ \\
\hline PB210 & 22.3 Years & $6 E-10$ & 8.0E-08 \\
\hline B1210 & 5.012 Days & $1.6 E-10$ & $8.0 E-08$ \\
\hline PO210 & 138:4 Days & $1.6 E-10$ & $.0 E-08$ \\
\hline CM245 & $8.499 \mathrm{kY}$ & $8.3 E-11$ & $4.3 E-08$ \\
\hline FR223 & 21.8 Min. & $4.5 E-11$ & $2.3 E-08$ \\
\hline NP236 & $115 \mathrm{kY}$ & $1.1 E-11$ & $5.9 E-09$ \\
\hline PO211 & 0.56 Sec. & $9.2 E-12$ & $4.7 E-09$ \\
\hline$\pi+229$ & $7.339 \mathrm{kY}$ & $2.1 E-12$ & 1.1E-09 \\
\hline$A C Z 25$ & 10 Days & $2.1 E-12$ & $1.1 E-09$ \\
\hline 81213 & $45.65 \mathrm{Min}$. & $2.1 E-12$ & $1.1 E-09$ \\
\hline AT217 & 0.032 Sec. & $2.1 E-12$ & $1.1 E-09$ \\
\hline PB209 & 3.3 Hours & $2.1 E-12$ & $1.1 \bar{E}-09$ \\
\hline FR221 & 4.8 Min. & $2.1 E-12$ & $1.1 E-09$ \\
\hline RA225 & 14.8 Days & $2.1 E-12$ & $1.1 E-09$ \\
\hline$P O 213$ & $4 E-06$ Soc. & $2.0 E-12$ & $1.0 E-09$ \\
\hline 246 & $4.731 \mathrm{kY}$ & $6.9 E-13$ & $3.6 E-10$ \\
\hline $\mathrm{TL}$ & $2.2 \mathrm{Min}$. & $4.4 E-14$ & $2.3 E-11$ \\
\hline TH232 & $14.05 \mathrm{GY}$ & $1.3 E-14$ & $6.6 \mathrm{E}-12$ \\
\hline
\end{tabular}


Table 2.3. (contd)

Table 3-E. Decay Time $=40$ Years

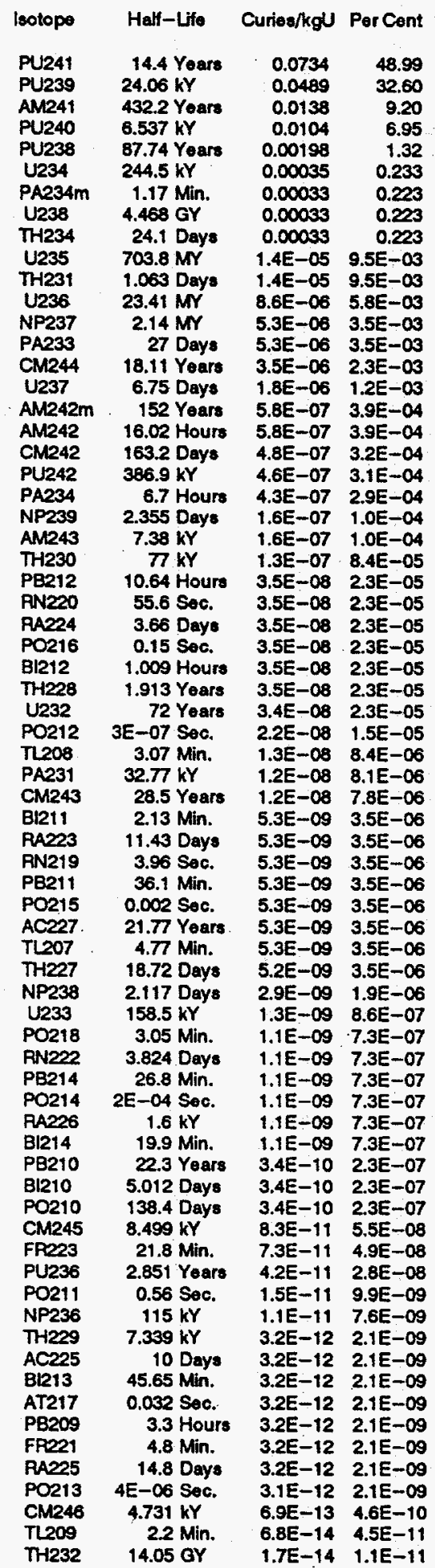

Table 3-F. Decay Time $=100$ Years

\begin{tabular}{|c|c|c|c|}
\hline tope & f-Life & $/ \mathrm{kgU}$ & Por C \\
\hline & & 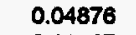 & \\
\hline & & & \\
\hline & & & \\
\hline & & & \\
\hline & & & \\
\hline & & & \\
\hline & & & .41 \\
\hline & & & \\
\hline & & & .414 \\
\hline & & & .018 \\
\hline & & & 0.018 \\
\hline U & & & 0.011 \\
\hline & & & 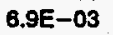 \\
\hline & & & \\
\hline & & & \\
\hline 242 & & & \\
\hline & & -07 & en \\
\hline & & & \\
\hline & $\therefore$ & -07 & 4.3E-O \\
\hline & & -07 & $3.9 \mathrm{E}-0$. \\
\hline & 2. & & \\
\hline & is & -07 & -0 \\
\hline & & & \\
\hline & & & BE-O \\
\hline & & & $E-0$ \\
\hline & Ye & & \\
\hline & De & -08 & -0 \\
\hline & & & \\
\hline & Mi & -08 & \\
\hline & & & \\
\hline & $N$ & & -0 \\
\hline & & & \\
\hline & & & -0 \\
\hline & D & & -0 \\
\hline & & & \\
\hline & s & & \\
\hline & & & \\
\hline & $Y_{0}$ & -08 & \\
\hline & & & \\
\hline & & & \\
\hline & & & \\
\hline & k & & \\
\hline & & & \\
\hline & & & \\
\hline & & & \\
\hline & $Y$ & & \\
\hline & & & \\
\hline & & & \\
\hline & & & \\
\hline & & & \\
\hline & & & \\
\hline & & & \\
\hline & & & \\
\hline & & & \\
\hline & & & \\
\hline & & & \\
\hline & & & \\
\hline & 0.0 & & \\
\hline & & & \\
\hline & & & \\
\hline & & & \\
\hline & 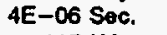 & -11 & $1.8 \mathrm{E}-\mathrm{c}$ \\
\hline & & & \\
\hline & & & \\
\hline & & & $8.5 E$ \\
\hline & & 13 & $3.9 \mathrm{E}$ \\
\hline & & & \\
\hline
\end{tabular}


Table 2.3. (contd)

Table 3-G. Decay Time $=300$ Years

\begin{tabular}{|c|c|c|c|}
\hline 010 & an- & $\mathrm{g}$ & n \\
\hline $\begin{array}{l}\text { PU239 } \\
\text { AM241 }\end{array}$ & 2 & & \\
\hline & & & 14.25 \\
\hline & & & \\
\hline $34 n$ & Ain. & & 0.470 \\
\hline & & 0.00033 & 0.470 \\
\hline & ays & 0.00033 & 0.470 \\
\hline & & & \\
\hline & & & 0.020 \\
\hline 35 & & & 0.020 \\
\hline & & & 0.012 \\
\hline & ay & & $9.0 E-03$ \\
\hline & & & $9.0 E-03$ \\
\hline & & -07 & $1.3 E-03$ \\
\hline & 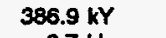 & -07 & $6.5 E-04$ \\
\hline & surs & -07 & -04 \\
\hline & & -07 & -04 \\
\hline $12 n$ & & -07 & -04 \\
\hline & & 07 & -04 \\
\hline 39 & & -07 & $2.2 E-04$ \\
\hline & & -07 & $2.2 E-04$ \\
\hline & & -07 & -04 \\
\hline 1 & & -08 & -04 \\
\hline & & 08 & \\
\hline & & 08 & $\begin{array}{l}-04 \\
-04\end{array}$ \\
\hline & & $\begin{array}{l}-08 \\
-08\end{array}$ & $\begin{array}{l}-04 \\
-04\end{array}$ \\
\hline & & -08 & 1. \\
\hline & & -08 & 1. \\
\hline & & & 1.1 \\
\hline & & .08 & 1.1 \\
\hline & & -08 & \\
\hline 218 & 3. & -08 & -05 \\
\hline & & -08 & 8. \\
\hline 214 & & -08 & 8.3 \\
\hline & & & 8.3 \\
\hline 214 & 2E- & -08 & 8.3E-05 \\
\hline & & -08 & -05 \\
\hline 210 & & -08 & 6.7 \\
\hline 210 & $y s$ & -08 & 6.7E-05 \\
\hline 233 & & $-\infty$ & -0 \\
\hline 216 & & -09 & 4. \\
\hline 1220 & c. & -09 & 4.0 \\
\hline 212 & 10. & -09 & 4.0 \\
\hline 12 & 8 & -09 & -06 \\
\hline 224 & & -09 & 4. \\
\hline 228 & ars & $-\infty$ & -06 \\
\hline & & .09 & 3. \\
\hline 212 & & 09 & 2. \\
\hline me & & 09 & -06 \\
\hline & & 09 & 1. \\
\hline & & 10 & 1. \\
\hline 21 & & 10 & 3. \\
\hline & & 10 & 77 \\
\hline & & 10 & -07 \\
\hline & & & -0 \\
\hline 225 & & -10 & -07 \\
\hline & & -10 & 1. \\
\hline & & -10 & -07 \\
\hline 200 & & 10 & -07 \\
\hline 213 & $4:$ & -10 & $1.6 E-07$ \\
\hline 1213 & & -10 & $1.6 E-07$ \\
\hline 1245 & & -11 & 1.1E-07 \\
\hline $1<4$ & 178 & -11 & $3.0 E-08$ \\
\hline 236 & & -11 & $1.6 E-08$ \\
\hline & & -12 & 9.3 \\
\hline 20 & 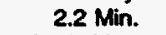 & -12 & $3.4 E-09$ \\
\hline 600 & & 16 & 1.4 \\
\hline & 4. & is & -10 \\
\hline & & 13 & 1.8 \\
\hline
\end{tabular}

Table 3-H. Decay Time $=1000$ Yeare

\begin{tabular}{|c|c|c|c|}
\hline stope & H-Lito & unie & Per Cen \\
\hline 238 & $\begin{array}{l}24.06 \mathrm{kY} \\
6.537 \mathrm{kY}\end{array}$ & $\begin{array}{l}0.04751 \\
0.00940\end{array}$ & $\begin{array}{l}6.85 \\
5.20\end{array}$ \\
\hline PU240 & oars & & 5.20 \\
\hline & & & \\
\hline & & & . \\
\hline & & 33 & 1.539 \\
\hline & $y e$ & 33 & .539 \\
\hline & & & \\
\hline & & & 0.02 \\
\hline & & & 0.014 \\
\hline & & & 0.013 \\
\hline & & -06 & 0.013 \\
\hline & $\pi$ & E-06 & $5.1 E-03$ \\
\hline & & $E-06$ & $\begin{array}{l}1.7 \mathrm{E}-0 \\
9.6 \mathrm{E}-0\end{array}$ \\
\hline & & & \\
\hline & 3.824 & $\begin{array}{l}=-07 \\
=-07 \\
=07\end{array}$ & $9.6 E-0$ \\
\hline & & & \\
\hline & & & \\
\hline & & $=-07$ & $9.6 E-0$ \\
\hline & & $\begin{array}{l}E-07 \\
E-07\end{array}$ & \\
\hline & & $=-07$ & \\
\hline & & $=-07$ & $7.5 E-0$ \\
\hline & & -07 & \\
\hline & & -07 & 4. \\
\hline & 21 & -07 & -0 \\
\hline & & & \\
\hline & 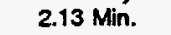 & -07 & $-c$ \\
\hline & & & \\
\hline & & & \\
\hline & 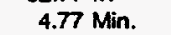 & & \\
\hline & 16 & -07 & \\
\hline & & & \\
\hline & & -07 & \\
\hline 33 & 15 & & \\
\hline & & & \\
\hline & $\pi$ & -09 & 1. \\
\hline & & & \\
\hline & & & \\
\hline & & & \\
\hline & $4.8 \mathrm{~K}$ & & \\
\hline & & & \\
\hline & & -09 & \\
\hline & $\mathbf{M}$ & .09 & \\
\hline & & & \\
\hline & $4 E$ & & 2 \\
\hline & & & \\
\hline & & & $\begin{array}{l}=0 \\
=-0\end{array}$ \\
\hline & 2.1 & -11 & 5.9E-C \\
\hline & & & $4.6 E$ \\
\hline & & & \\
\hline & 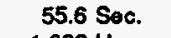 & -12 & $7.9 E-C$ \\
\hline & 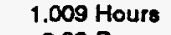 & & $795-$ \\
\hline & & & \\
\hline & & & $7.9 \mathrm{E}-\mathrm{C}$ \\
\hline & $H$ & & $7.9 \mathrm{E}-1$ \\
\hline & & & \\
\hline & & & \\
\hline 212 & $3 E-07$ & $3.1 E-12$ & $5.0 E-$ \\
\hline & & & \\
\hline & 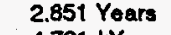 & & \\
\hline $\begin{array}{l}\text { CM2 } \\
\mathrm{RA2}\end{array}$ & $6.7 Y$ & $\begin{array}{l}13 \\
13\end{array}$ & \\
\hline & & & \\
\hline & & -13 & $7.0 \mathrm{E}$ \\
\hline & & 11 & \\
\hline
\end{tabular}


Table 2.3. (contd)

Table 3-I. Decay Time $=3000$ Yoars

\begin{tabular}{|c|c|c|c|}
\hline tope & Half-Life & Curies/kgu & Per Cont \\
\hline $\begin{array}{l}\text { PU239 } \\
\text { PU240 }\end{array}$ & $\begin{array}{l}24.06 \mathrm{kY} \\
6.537 \mathrm{kY}\end{array}$ & $\begin{array}{l}0.04485 \\
0.00760\end{array}$ & $\begin{array}{l}82.97 \\
14.07\end{array}$ \\
\hline U234 & $244.5 \mathrm{kY}$ & 0.00035 & 0.647 \\
\hline U238 & $4.468 \mathrm{GY}$ & 0.00033 & 0.617 \\
\hline PA234m & 1.17 Min. & 0.00033 & 0.617 \\
\hline TH234 & 24.1 Days & 0.00033 & 0.617 \\
\hline AM241 & 432.2 Years & 0.00014 & 0.262 \\
\hline U235 & $703.8 \mathrm{MY}$ & $1.4 E-05$ & 0.027 \\
\hline TH231 & 1.063 Days & $1.4 E-05$ & 0.027 \\
\hline U236 & $23.41 \mathrm{MY}$ & $9.4 E-06$ & 0.017 \\
\hline TH230 & $77 \mathrm{kY}$ & 9.3E-06 & 0.017 \\
\hline & $\begin{array}{l}27 \text { Days } \\
214 \mathrm{M}\end{array}$ & $.5 E-\infty 6$ & 0.016 \\
\hline $\begin{array}{l}\text { NP237 } \\
\text { RN2222 }\end{array}$ & $\begin{array}{l}2.14 \mathrm{MV} \\
3.824 \text { Days }\end{array}$ & $\begin{array}{l}8.5 E-06 \\
4.1 E-06\end{array}$ & $\begin{array}{r}0.016 \\
7.6 E-03\end{array}$ \\
\hline PA226 & $1.6 \mathrm{kY}$ & $4.1 \mathrm{E}-06$ & $\begin{array}{l}7.0 E-03 \\
7.6 E-03\end{array}$ \\
\hline PO218 & $3.05 \mathrm{Min}$. & $4.1 E-06$ & $7.6 \mathrm{E}-03$ \\
\hline PB214 & 26.8 Min. & $4.1 E-06$ & $7.6 E-03$ \\
\hline Bi214 & 19.9 Min. & 4.1E-06 & $\begin{array}{r}7,6 E-03 \\
7.6 E-03\end{array}$ \\
\hline $\begin{array}{l}\text { PO210 } \\
\text { PB210 }\end{array}$ & $\begin{array}{c}138.4 \text { Days } \\
22.3 \text { Years }\end{array}$ & & $\begin{array}{l}7.6 E-03 \\
7.6 E-03\end{array}$ \\
\hline $\begin{array}{l}\text { P8210 } \\
\text { PO214 }\end{array}$ & 2E-04 Sec. & $\begin{array}{l}4.1 E-06 \\
4.1 E-06\end{array}$ & $\begin{array}{l}7.6 E-03 \\
7.6 E-03\end{array}$ \\
\hline 81210 & 5.012 Days & $4.1 E-06$ & $7.6 \mathrm{E}-03$ \\
\hline $\begin{array}{l}\text { AC227 } \\
\text { PO215 }\end{array}$ & $\begin{array}{l}21.77 \text { Yoars } \\
0.002 \text { Soc. }\end{array}$ & $\begin{array}{l}8.8 E-07 \\
8.8 E-07\end{array}$ & $\begin{array}{l}1.6 E-03 \\
1.6 E-03\end{array}$ \\
\hline $\begin{array}{l}\text { PO215 } \\
\text { RN219 }\end{array}$ & 3.96 Soc. & $\begin{array}{l}8.8 E-07 \\
8.8 E-07\end{array}$ & $\begin{array}{l}1.6 E-03 \\
1.6 E-03\end{array}$ \\
\hline Bl211 & 2.13 Min. & $8.8 \mathrm{E}-07$ & $1.6 E-03$ \\
\hline RA223 & 11.43 Days & $8.8 E-07$ & $1.6 \mathrm{E}-03$ \\
\hline $\begin{array}{l}\text { PB211 } \\
\text { PA231 }\end{array}$ & $\begin{array}{l}36.1 \text { Min. } \\
3277 \text { KY }\end{array}$ & $8.8 E-07$ & $1.6 \mathrm{E}-03$ \\
\hline $\begin{array}{l}\text { PA231 } \\
\text { TL207 }\end{array}$ & $4.77 \mathrm{Min}$. & $\begin{array}{l}8.8 E-07 \\
8.8 E-07\end{array}$ & $\begin{array}{l}1.6 \mathrm{E}-03 \\
16 \mathrm{E}-03\end{array}$ \\
\hline TH2227 & 18.72 Days & $8.7 E-07$ & $1.6 E-03$ \\
\hline PU242 & $386.9 \mathrm{kY}$ & $4.6 \mathrm{E}-07$ & $8.5 E-04$ \\
\hline PA234 & 6.7 Hours & $4.3 E-07$ & 8.0E-04 \\
\hline AM1243 & & $\begin{array}{l}1.2 E-07 \\
1.2 E-07\end{array}$ & $\begin{array}{l}2.2 E-04 \\
2.2 E-04\end{array}$ \\
\hline $\begin{array}{l}\text { NP2339 } \\
\text { U233 }\end{array}$ & $\begin{array}{l}2.3555 \text { Days } \\
158.5 \mathrm{kY}\end{array}$ & $1.0 E-07$ & $\begin{array}{l}2.2 E-04 \\
1.9 E-04\end{array}$ \\
\hline AC225 & 10 Days & $1.3 E-08$ & 2.3E-05 \\
\hline RA225 & 14.8 Days & $1.3 E-08$ & 2.3E-05 \\
\hline TH229 & $7.339 \mathrm{kY}$ & 1.3E-08 & 2.3E-05 \\
\hline PB209 & 3.3 Hours & 1.3E-08 & 2.3E-05 \\
\hline AT217 & 0.032 Sec. & $1.3 E-08$ & 2.3E-05 \\
\hline FPE21 1 & 4.8 Min. & $1.3 E-08$ & 2.3E-05 \\
\hline B1213 & $45.65 \mathrm{Min}$. & $1.3 E-08$ & 2.3E-05 \\
\hline PO213 & $4 E-06$ Sec. & 1.2E- -08 & 2.3E-05 \\
\hline FP223 & 21.8 Min. & $1.2 E-08$ & $2.2 E-05$ \\
\hline PO211 & 0.56 Sec. & $2.5 \mathrm{E}-\infty 9$ & $4.6 E-06$ \\
\hline TL209 & 2.2 Min. & $2.7 E-10$ & 5.1E-07 \\
\hline PU241 & 14.4 Years & $6.5 E-11$ & $1.2 E-07$ \\
\hline CM245 & $8.499 \mathrm{kV}$ & $6.5 E-11$ & $1.2 E-07$ \\
\hline NP236 & $115 \mathrm{kY}$ & $1.1 E-11$ & $2.1 \varepsilon-08$ \\
\hline Bl212 & 1.009 Hours & 2.3E-12 & $4.3 E-09$ \\
\hline RA224 & 3.66 Days & 2.3E-12 & 4.3E -09 \\
\hline PB212 & 10.64 Hours & 2.3E-12 & 4.3E -09 \\
\hline RN220 & 55.6 & 2.3E-12 & $4.3 E-09$ \\
\hline TH 228 & 1.913 Years & $2.3 E-12$ & $4.3 E-09$ \\
\hline PO216 & 0.15 So & $2.3 E-12$ & $4.3 E-09$ \\
\hline PU238 & 87.74 Yoars & $1.7 E-12$ & $3.2 E-09$ \\
\hline PO212 & 3E-07 Soc. & $1.5 E-12$ & $2.8 E-09$ \\
\hline TH232 & $14.05 \mathrm{GY}$ & 1.3E-12 & $2.5 E-09$ \\
\hline AC228 & 6.131 Hours & $1.3 E-12$ & 2.5E-09 \\
\hline RA228 & 6.7 Years & $1.3 E-12$ & $2.5 E-09$ \\
\hline PU236 & 2.851 Yoars & $1.0 E-12$ & $1.9 E-\infty 9$ \\
\hline U232 & 72 Years & $1.0 E-12$ & $1.9 E-09$ \\
\hline TL208 & $3.07 \mathrm{Min}$. & $8.4 E-13$ & $1.6 \mathrm{E}-09$ \\
\hline AM242m & $152 \mathrm{Ye}$ & $8.0 E-13$ & $1.5 E-09$ \\
\hline AM242 & 16.02 Hours & $8.0 E-13$ & $1.5 E-09$ \\
\hline CM242 & 163.2 Days & $6.6 E-13$ & $1.2 E-09$ \\
\hline CM246 & $4.731 \mathrm{kY}$ & $4.5 E-13$ & $8.3 E-10$ \\
\hline $\begin{array}{l}\text { NP238 } \\
\text { PU244 }\end{array}$ & $\begin{array}{l}2.117 \text { Days } \\
82.61 \mathrm{MY}\end{array}$ & $\begin{array}{l}4.0 E-15 \\
2.5 E-15\end{array}$ & $7.4 E-12$ \\
\hline & 82. & $2.5 E-15$ & $4.5 E-12$ \\
\hline
\end{tabular}


Table 2.4. Relative Inhalation Hazard of Fission Products in Hanford Reactor Fuel at Various Decay Times

Tabio 4-A. Decay Time $=1$ Year

\begin{tabular}{|c|c|c|c|}
\hline Isotope & Helt-Lito & Alr Hazard & PorCor \\
\hline $\begin{array}{l}\text { CE144 } \\
\text { RU106 }\end{array}$ & $\begin{array}{l}284.3 \text { Days } \\
1.008 \text { Years }\end{array}$ & $\begin{array}{l}9.81 E+11 \\
1.97 E+11\end{array}$ & $\begin{array}{l}77.23 \\
15.52\end{array}$ \\
\hline SR90 & 29.12 Years & $4.08 E+10$ & 3.21 \\
\hline PM147 & 2.623 Years & $1.83 E+10$ & 1.44 \\
\hline $\begin{array}{l}\text { Y91 } \\
\text { ZR } 95\end{array}$ & $\begin{array}{l}58.51 \text { Days } \\
63.98 \text { Days }\end{array}$ & $\begin{array}{l}1.14 E+10 \\
6.03 E+\infty 9\end{array}$ & $\begin{array}{l}0.894 \\
0.475\end{array}$ \\
\hline CS137 & 30 Years & $5.95 E+\infty 9$ & 0.468 \\
\hline $\begin{array}{l}\text { NB } 95 \\
Y 90\end{array}$ & $\begin{array}{l}35.15 \text { Days } \\
2.667 \text { Days }\end{array}$ & $\begin{array}{l}3.90 E+09 \\
2.04 E+09\end{array}$ & $\begin{array}{l}0.307 \\
0.161\end{array}$ \\
\hline $\operatorname{cs} 134$ & 2.052 Years & $1.02 E+09$ & 0.080 \\
\hline SR 89 & 50.5 Days & $7.51 E+08$ & 0.059 \\
\hline SE125 & 2.77 Years & $3.25 E+08$ & 0.026 \\
\hline EU154 & 8.6 Years & $2.78 E+08$ & 0.022 \\
\hline TE127m & 109 Days & $2.01 E+08$ & 0.016 \\
\hline RU103 & 39.28 Days & $1.88 E+08$ & 0.015 \\
\hline EU155 & 4.959 Years & $1.87 E+08$ & 0.015 \\
\hline CE141 & 32.51 Days & $1.58 E+08$ & 0.012 \\
\hline $\begin{array}{l}\text { SN123 } \\
\text { CD113m }\end{array}$ & $\begin{array}{l}\text { 129.2 Days } \\
14.59 \text { Years }\end{array}$ & $\begin{array}{l}1.21 E+08 \\
1.16 E+08\end{array}$ & $\begin{array}{r}0.010 \\
9.1 E-03\end{array}$ \\
\hline SM151 & 89.99 Years & $1.11 E+O B$ & $8.7 E-03$ \\
\hline $\begin{array}{l}\text { PR144 } \\
\text { TE125m }\end{array}$ & $\begin{array}{l}\text { 17.28 Min. } \\
53 \text { Days }\end{array}$ & $\begin{array}{l}9.81 E+07 \\
3.92 E+07\end{array}$ & $\begin{array}{l}7.7 E-03 \\
3.1 E-03\end{array}$ \\
\hline $\mathrm{NB} 95 \mathrm{~m}$ & 3.609 Days & $8.05 E+06$ & $6.3 E-04$ \\
\hline TE129m & 33.6 Days & $7.52 E+06$ & 5.9E-04 \\
\hline AG110m & 249.9 Days & $6.28 E+06$ & 4.9E-04 \\
\hline CD115m & 44.59 Days & $5.38 E+06$ & $4.2 E-04$ \\
\hline PM148m & 41.3 Days & $4.36 E+06$ & $3.4 E-04$ \\
\hline TE127 & 9.35 Hours & $2.95 E+06$ & 2.3E-04 \\
\hline SN119m & 245 Days & $2.12 E+06$ & $1.7 E-04$ \\
\hline EU152 & 13.6 Years & $1.02 E+06$ & 8.0E-OS \\
\hline ZR 93 & $1.53 \mathrm{MY}$ & $4.72 E+05$ & $3.7 E-05$ \\
\hline SB124 & 60.2 Days & $1.73 E+05$ & $1.4 E-05$ \\
\hline PM148 & 5.37 Days & $1.72 E+05$ & $1.4 E-05$ \\
\hline TC 99 & $213 \mathrm{kY}$ & $1.72 E+05$ & $1.4 E-05$ \\
\hline TB160 & 72.3 Days & $1.19 E+05$ & $9.4 E-06$ \\
\hline H 3 & 12.35 Yeats & $1.17 E+05$ & $9.2 E-06$ \\
\hline $\mathrm{RH} 103 \mathrm{~m}$ & $56.12 \mathrm{Min}$. & $1.13 E+05$ & $8.9 E-06$ \\
\hline SN126 & $100 \mathrm{kY}$ & $8.25 E+04$ & $6.5 E-05$ \\
\hline PM146 & 5.5 Years & $5.02 E+04$ & $4.0 E-06$ \\
\hline TE129 & 1.16 Hours & $1.47 E+04$ & $1.2 E-05$ \\
\hline GD153 & 242 Days & $1.25 \mathrm{E}+04$ & $9.8 E-07$ \\
\hline SE 79 & $64.96 \mathrm{kY}$ & $1.02 E+04$ & 8.0E-07 \\
\hline 1129 & $15.7 \mathrm{Mr}$ & 9678 & $7.6 E-07$ \\
\hline $\mathrm{RH} 102$ & 2.9 Years & 5114 & $4.0 E-07$ \\
\hline SNi21m & 49.97 Years & 3027 & $2.4 E-07$ \\
\hline $\operatorname{cs} 135$ & $2.3 \mathrm{MV}$ & 2775 & $2.2 E-07$ \\
\hline SB126 & 12.4 Days & 2310 & $1.8 E-07$ \\
\hline PR143 & 13.56 Days & 1956 & $1.5 E-07$ \\
\hline P0107 & $6.496 \mathrm{Mr}$ & 1340 & $1.1 E-07$ \\
\hline NB 93m & 13.6 Years & 622 & $4.9 E-08$ \\
\hline BA140 & 12.79 Days & 412 & $3.2 \mathrm{E}-08$ \\
\hline EU156 & 15.19 Days & 401 & $3.2 \mathrm{E}-0.8$ \\
\hline LA140 & 1.676 Days & 356 & $2.8 E-08$ \\
\hline SM147 & $107 \mathrm{GY}$ & 291 & $2.3 E-0.8$ \\
\hline TE123m & 119.7 Days & 202 & $1.6 E-08$ \\
\hline HO166m & $1.2 \mathrm{kY}$ & 53 & 4.1E-09 \\
\hline IN114m & 49.51 Days & 49 & $3.9 E-09$ \\
\hline NB 94 & $20.3 \mathrm{kY}$ & 39 & $3.1 \mathrm{E}-09$ \\
\hline $\mathrm{SB} 126 \mathrm{~m}$ & 19 Min. & 33 & $2.6 E-09$ \\
\hline RB 86 & 18.66 Days & 19 & $1.5 E-09$ \\
\hline ND147 & 11.06 Days & 11 & $8.3 E-10$ \\
\hline PH106 & 29.9 Soc. & 5.9 & $4.7 E-10$ \\
\hline CS136 & 13.1 Days & 4.9 & $3.8 E-10$ \\
\hline $\mathrm{BA} 137 \mathrm{~m}$ & $2.552 \mathrm{Min}$. & 2.3 & $1.8 E-10$ \\
\hline BE 10 & $1.6 \mathrm{Mr}$ & 2.2 & $1.8 \mathrm{E}-10$ \\
\hline IN115m & 4.3 Hours & 0.76 & $5.9 E-11$ \\
\hline$A G 108 m$ & 127 Years & 0.56 & $4.4 E-11$ \\
\hline C 14 & $5.729 \mathrm{kY}$ & 0.45 & $3.6 E-11$ \\
\hline $\operatorname{Sin} 146$ & $70 \mathrm{MN}$ & 0.38 & $3.0 E-11$ \\
\hline PR144m & $7.2 \mathrm{Min}$. & 0.35 & $2.8 E-11$ \\
\hline
\end{tabular}

Table 4-B. Decay Time $=3$ Yoaro

\begin{tabular}{|c|c|c|c|}
\hline lsotope & Hall-Lie & Air Hazard & Per Cont \\
\hline $\begin{array}{l}\text { CE144 } \\
\text { RU106 }\end{array}$ & $\begin{array}{l}284.3 \text { Days } \\
1.008 \text { Yoars }\end{array}$ & $\begin{array}{l}1.65 E+11 \\
4.98 E+10\end{array}$ & $\begin{array}{l}60.37 \\
18.20\end{array}$ \\
\hline $\begin{array}{l}\text { AU105 } \\
\text { SR } 90\end{array}$ & 29.12 Yoars & $3.89 E+10$ & $\begin{array}{l}18.20 \\
14.20\end{array}$ \\
\hline PMI47 & 2.623 Years & $1.08 E+10$ & 3.94 \\
\hline $\operatorname{cs} 137$ & 30 Years & $5.68 E+09$ & 2.07 \\
\hline$Y 90$ & 2.667 Days & $1.95 E+09$ & 0.710 \\
\hline $\operatorname{cs} 134$ & 2.062 Years & $5.21 E+\infty 8$ & 0.190 \\
\hline EU154 & 8.6 Yoare & $2.37 E+08$ & 0.087 \\
\hline$S B 125$ & 2.77 Years & $1.97 E+08$ & 0.072 \\
\hline EU155 & 4.959 Years & $1.42 E+08$ & 0.052 \\
\hline SM151 & 89.99 Yeare & $1.09 E+08$ & 0.040 \\
\hline CD113m & 14.59 Years & $1.06 \mathrm{E}+08$ & 0.039 \\
\hline TE125m & 58 Days & 2.40E+07 & $8.8 E-03$ \\
\hline PR144 & 17.28 Min. & $1.65 E+07$ & $6.0 E-03$ \\
\hline $\begin{array}{l}\text { SN123 } \\
\text { ZR95 }\end{array}$ & $\begin{array}{l}129.2 \text { Days } \\
63.98 \text { Days }\end{array}$ & $\begin{array}{l}2.40 E+06 \\
2.21 E+06\end{array}$ & $\begin{array}{l}8.8 E-04 \\
8.1 E-04\end{array}$ \\
\hline Y 91 & 58.51 Days & $1.98 E+06$ & $7.2 E-04$ \\
\hline$T E 127 m$ & 109 Days & $1.93 E+06$ & $7.0 E-04$ \\
\hline NB 95 & 35.15 Days & $1.47 E+06$ & 5.4E-0.4 \\
\hline EU152 & 13.6 Years & $9.23 E+05$ & $3.4 E-04$ \\
\hline $\begin{array}{l}\text { AG110m } \\
\text { ZA93 }\end{array}$ & $\begin{array}{l}249.9 \text { Days } \\
1.53 \mathrm{MY}\end{array}$ & $\begin{array}{l}8.27 E+05 \\
4.72 E+05\end{array}$ & $\begin{array}{l}3.0 E-04 \\
1.7 E-04\end{array}$ \\
\hline SN119m & 245 Days & $2.69 E+05$ & $9.8 E-05$ \\
\hline TC 99 & $213 \mathrm{kY}$ & $1.72 E+05$ & $6.3 E-05$ \\
\hline H 3 & 12.35 Years & $1.05 E+05$ & $3.8 E-05$ \\
\hline SN126 & $100 \mathrm{kY}$ & $8.25 E+04$ & $3.0 E-05$ \\
\hline PMI 46 & 5.5 Years & $3.90 E+04$ & $1.4 E-05$ \\
\hline SA 89 & 50.5 Days & $3.32 E+04$ & $1.2 E-05$ \\
\hline TE127 & 9.35 Hours & $2.84 E+04$ & $1.0 E-05$ \\
\hline SE 79 & $64.96 \mathrm{kY}$ & $1.02 E+04$ & $3.7 E-06$ \\
\hline 1129 & $15.7 \mathrm{MY}$ & 9678 & $3.5 E-06$ \\
\hline RH102 & 2.9 Years & 3170 & $1.2 E-06$ \\
\hline SN121m & 49.97 Years & 2945 & $1.1 E-06$ \\
\hline NB $95 \mathrm{~m}$ & 3.609 Days & 2944. & $1.1 E-06$ \\
\hline CS135 & $2.3 \mathrm{MY}$ & 2775 & $1.0 E-06$ \\
\hline SB126 & 12.4 Days & 2309 & $8.4 E-07$ \\
\hline NB $93 \mathrm{~m}$ & 13.6 Years & 1648 & $6.0 E-07$ \\
\hline GD153 & 242 Days & 1543 & $5.6 E-07$ \\
\hline PD107 & $6.496 \mathrm{MY}$ & 1340 & $4.9 E-07$ \\
\hline SMI47 & $107 \mathrm{GY}$ & 660 & 2.4E- 07 \\
\hline RU103 & 39.28 Days & 474 & $1.7 E-07$ \\
\hline TB160 & 72,3 Days & 109 & $4.0 E-08$ \\
\hline $\mathrm{CD} 115 \mathrm{~m}$ & 44.59 Days & 63 & $2.3 E-08$ \\
\hline HO166m & $1.2 \mathrm{kY}$ & 53 & $1.9 E-08$ \\
\hline NB 94 & $20.3 \mathrm{kY}$ & 39 & $1.4 E-08$ \\
\hline SB124 & 60.2 Days & 38 & $1.4 E-08$ \\
\hline $\mathrm{SB1} 26 \mathrm{~m}$ & $19 \mathrm{Min}$. & 33 & $1.2 E-08$ \\
\hline CE141 & 32.51 Days & 27 & $9.9 E-09$ \\
\hline PM148m & 41.3 Days & 21 & $7.5 E-09$ \\
\hline TE123m & 119.7 Days & 2.9 & $1.1 E-09$ \\
\hline BE 10 & $1.6 \mathrm{Mr}$ & 2.2 & $8.2 E-10$ \\
\hline BA137m & $2.552 \mathrm{Min}$. & 2.1 & $7.8 \mathrm{E}-10$ \\
\hline TE129m & 33.6 Days & 2.1 & $7.8 E-10$ \\
\hline $\mathrm{PH} 106$ & 29.9 Sec. & 1.5 & $5.5 E-10$ \\
\hline PMI48 & 5.37 Days & 0.81 & $3.0 E-10$ \\
\hline SM146 & $70 \mathrm{Mr}$ & 0.71 & $2.6 E-10$ \\
\hline AG108m & 127 Years & 0.56 & $2.0 E-10$ \\
\hline C 14 & $5.729 \mathrm{kY}$ & 0.45 & 1.7E-10 \\
\hline $\mathrm{RH} 103 \mathrm{~m}$ & $56.12 \mathrm{Min}$. & 0.28 & $1.0 E-10$ \\
\hline KA 85 & 10.72 Years & 0.23 & $8.2 E-11$ \\
\hline RB 87 & $46.96 \mathrm{GY}$ & 0.15 & $5.4 E-11$ \\
\hline CD109 & 1.27 Years & 0.11 & $4.1 E-11$ \\
\hline PA144m & 7.2 Min. & 0.059 & $2.2 E-11$ \\
\hline TC 98 & $4.199 \mathrm{MY}$ & $8.4 E-03$ & $3.1 E-12$ \\
\hline TE129 & 1.16 Hours & $4.2 E-03$ & $1.5 E-12$ \\
\hline EU150 & 36 Years & $1.9 E-03$ & $7.1 E-13$ \\
\hline INI14m & 49.51 Days & $1.8 E-03$ & $6.5 E-13$ \\
\hline TM170 & 128.6 Days & $1.8 \mathrm{E}-03$ & $6.5 E-13$ \\
\hline LA138 & $135 \mathrm{GY}$ & $5.2 E-04$ & $1.9 E-13$ \\
\hline IN115 & $5 E+05 G Y$ & $4.3 E-04$ & $1.6 E-13$ \\
\hline
\end{tabular}


Table 2.4. (contd)

Tablo 4-C. Decay Time -10 Yoars

\begin{tabular}{|c|c|c|c|}
\hline De & H-Lifo & zard & \\
\hline 890 & $\begin{array}{l}29.12 \text { Years } \\
30 \text { Years }\end{array}$ & $\begin{array}{l}3.29 E+10 \\
4.83 E+09\end{array}$ & \\
\hline 7 & 2.623 Years & $1.70 E+09$ & \\
\hline$Y 90$ & 667 Days & $1.65 E+09$ & \\
\hline U106 & 208 Yó & $4.05 E+08$ & \\
\hline & 284.3 Days & $3.24 E+08$ & \\
\hline$F$ & $8.6 Y$ & & \\
\hline & & & \\
\hline c & $9 \gamma$ & & \\
\hline 115 & is & +07 & 0.1 \\
\hline & & & \\
\hline & ars & 3.4 & 0.0 \\
\hline & & & \\
\hline & & & 1. \\
\hline & & & \\
\hline TC 99 & 2 & & \\
\hline SN126 & & & \\
\hline & & & \\
\hline & 17.28 & & \\
\hline & & & \\
\hline & 64.5 & & \\
\hline & & 678 & \\
\hline NB & & & \\
\hline $\operatorname{cs} 1$ & & & \\
\hline & & & \\
\hline & & 2309 & \\
\hline & & 1340 & \\
\hline SM1 & & 1106 & 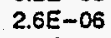 \\
\hline & & 88 & \\
\hline & & 95 & \\
\hline & & 194 & \\
\hline & & 52 & 1.2 \\
\hline 8 & & 39 & $9.2 E-C$ \\
\hline & & 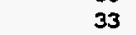 & 78 \\
\hline & 129 & 2.6 & \\
\hline & & 22 & \\
\hline & & .8 & \\
\hline & & .4 & \\
\hline & & .0 & \\
\hline & & 0.54 & \\
\hline & & 45 & \\
\hline & & 7 & \\
\hline & & 15 & \\
\hline & & & \\
\hline $\mathrm{HI}$ & & & \\
\hline & & & \\
\hline$D 18$ & & & 1. \\
\hline & & & \\
\hline & & & 4. \\
\hline & & & \\
\hline & & & \\
\hline & & & \\
\hline & $I E+C$ & & \\
\hline & & & \\
\hline & & & \\
\hline & & & \\
\hline 12 & & & \\
\hline & & & \\
\hline & 3. & & \\
\hline & & & \\
\hline & & & \\
\hline & & & \\
\hline & & & \\
\hline & & & \\
\hline & 23 & & \\
\hline & & & \\
\hline & 0.2 Days & & $1.5 E-26$ \\
\hline & 205 & -12 & $5.5 E-2$ \\
\hline & & & \\
\hline & & & \\
\hline
\end{tabular}

Table 4-D. Docay Time = 30 Yoars

\begin{tabular}{|c|c|c|c|}
\hline Isolope & Haff-Lito & Air Hezard & Por Cent \\
\hline $\begin{array}{l}\text { SR } 90 \\
\text { CS137 }\end{array}$ & $\begin{array}{r}29.12 \text { Yosis } \\
30 \text { Yoars }\end{array}$ & $\begin{array}{l}2.05 E+10 \\
3.04 E+09\end{array}$ & $\begin{array}{l}82.88 \\
12.33\end{array}$ \\
\hline$Y 90$ & 2.667 Days & $1.02 E+09$ & $\begin{array}{r}4.15 \\
0.359\end{array}$ \\
\hline SM151 & 89.99 Yoars & $8.87 E+07$ & 0.359 \\
\hline $\begin{array}{l}\text { CD113m } \\
\text { EU154 }\end{array}$ & $\begin{array}{r}14.59 \text { Yoaro } \\
8.6 \text { Yoars }\end{array}$ & $\begin{array}{l}2.93 E+07 \\
2.69 E+07\end{array}$ & $\begin{array}{l}0.119 \\
0.109\end{array}$ \\
\hline PM147 & 2.623 Yoars & $8.62 E+06$ & 0.035 \\
\hline EU155 & 4.959 Years & $3.25 E+06$ & 0.013 \\
\hline ZR 93 & $1.53 \mathrm{KN}$ & $4.72 E+05$ & $1.9 E-03$ \\
\hline EU152 & 13.6 Years & $2.33 E+05$ & $9.5 E-0.4$ \\
\hline SB125 & 2.77 Yoars & $2.29 E+05$ & 9.3E-04 \\
\hline TC 99 & $213 \mathrm{kY}$ & $1.72 E+05$ & $7.0 E-04$ \\
\hline SN126 & $100 \mathrm{kY}$ & 8.25E+04 & 3.3E-04 \\
\hline CS134 & 2.062 Yoars & $5.97 E+04$ & $2.4 E-04$ \\
\hline TE125m & 58 Days & $2.79 E+04$ & $1.1 E-04$ \\
\hline $\begin{array}{l}\text { H } 3 \\
\text { SE } 79\end{array}$ & 12.35 Yoars & $\begin{array}{l}2.30 E+04 \\
1.02 E+04\end{array}$ & $\begin{array}{l}9.3 E-05 \\
4.1 E-05\end{array}$ \\
\hline $\begin{array}{l}\text { SE } 79 \\
1129\end{array}$ & $\begin{array}{l}64.96 \mathrm{KY} \\
15.7 \mathrm{MV}\end{array}$ & $\begin{array}{r}1.02 E+0.4 \\
9678\end{array}$ & $\begin{array}{l}4.1 E-05 \\
3.9 E-05\end{array}$ \\
\hline NB 93m & 13.6 Yoars & 8794 & $3.6 E-05$ \\
\hline CS135 & $2.3 \mathrm{Mr}$ & 2775 & $1.1 E-05$ \\
\hline SB126 & 12.4 Days & 2309 & $9.4 E-06$ \\
\hline SN121m & 49.97 Yeats & 2025 & $8.2 E-06$ \\
\hline P0107 & $6.496 \mathrm{Mr}$ & 1340 & $5.4 E-06$ \\
\hline PM146 & 5.5 Years & 1299 & $5.3 E-06$ \\
\hline SM147 & $107 \mathrm{GY}$ & 1189 & $4.8 E-06$ \\
\hline RU106 & 1.008 Years & 431 & $1.7 E-06$ \\
\hline HO166m & $1.2 \mathrm{kY}$ & 52 & $2.1 E-07$ \\
\hline NB 94 & $20.3 \mathrm{kY}$ & 39 & $1.6 E-07$ \\
\hline SB126m & $19 \mathrm{Min}$. & 33 & 1.3E-07 \\
\hline CE144 & 284.3 Days & 6.0 & $2.4 E-08$ \\
\hline RH102 & 2.9 Years & $\begin{array}{l}5.0 \\
2.2\end{array}$ & 2.0E-08 \\
\hline BE 10 & $1.6 \mathrm{Mr}$ & 2.2 & $9.1 E-09$ \\
\hline SM146 & $70 \mathrm{MY}$ & 1.8 & 7.3E-09 \\
\hline BA137m & $2.552 \mathrm{Min}$. & 1.2 & $4.7 E-09$ \\
\hline AG108m & 127 Years & 0.48 & $1.9 E-09$ \\
\hline C 14 & $5.729 \mathrm{kY}$ & 0.45 & $1.8 E-09$ \\
\hline RB 87 & $46.96 \mathrm{GY}$ & 0.15 & $6.0 E-10$ \\
\hline KA 85 & 10.72 Years & 0.039 & $1.6 E-10$ \\
\hline TC 98 & $4.199 \mathrm{Mr}$ & $8.4 \mathrm{E}-03$ & $3.4 E-11$ \\
\hline EU150 & 36 Years & $1.1 E-03$ & $4.7 E-12$ \\
\hline PR144 & $17.28 \mathrm{Min}$. & $6.0 E-04$ & $2.4 E-12$ \\
\hline LA138 & & $5.2 E-04$ & $2.1 E-12$ \\
\hline IN115 & $5 E+05 \mathrm{GY}$ & 4.3E-04 & $1.8 E-12$ \\
\hline GD152 & $I E+05 G Y$ & $8.4 E-05$ & $3.4 E-13$ \\
\hline$A G 110 \mathrm{~m}$ & 249.9 Days & $1.1 E-06$ & $4.4 E-15$ \\
\hline SN119m & 245 Days & $2.1 E-07$ & $8.3 E-16$ \\
\hline CD109 & 1.27 Years & $4.5 E-08$ & $1.8 E-16$ \\
\hline RH106 & $29.9 \mathrm{Sec}$. & 1.3E-08 & $5.2 E-17$ \\
\hline TM171 & 1.92 Years & $5.0 E-09$ & $2.0 E-17$ \\
\hline GD153 & 242 Days & $8.4 E-10$ & $3.4 E-18$ \\
\hline CE142 & $104.9 \mathrm{GY}$ & $6.5 E-10$ & $2.6 E-18$ \\
\hline TE123 & $10001 \mathrm{GY}$ & $7.4 E-11$ & $3.0 E-19$ \\
\hline AG108 & 2.37 Min. & $1.7 E-11$ & $6.9 E-20$ \\
\hline KR 81 & $209.9 \mathrm{kr}$ & $2.3 E-12$ & $9.5 E-21$ \\
\hline PRI 44m & 7.2 Min. & $2.1 E-12$ & $8.7 E-21$ \\
\hline ND144 & $2 E+06 \mathrm{GY}$ & $3.0 E-14$ & $1.2 \mathrm{E}-22$ \\
\hline $\sin 149$ & $1 E+07 \mathrm{GY}$ & $1.8 E-16$ & $7.4 E-25$ \\
\hline SM148 & $8 E+06 \mathrm{GY}$ & $1.4 E-16$ & $5.9 E-25$ \\
\hline SN123 & 129.2 Days & $2.5 E-17$ & $1.0 E-25$ \\
\hline AG109m & $39.6 \mathrm{Sec}$. & $4.5 E-18$ & $1.8 E-26$ \\
\hline AG110 & 24,6 Soc. & $4.3 E-18$ & $1.8 \mathrm{E}-26$ \\
\hline TE127m & 109 & $1.1 E-21$ & $4.6 \mathrm{E}-30$ \\
\hline TE127 & 9.35 Hours & $1.7 E-23$ & $6.7 E-32$ \\
\hline TE123m & 119.7 Days & $4.6 \mathrm{E}-25$ & $1.9 \bar{E}-33$ \\
\hline TM170 & 128.6 Days & $1.5 \mathrm{E}-26$ & $5.9 E-35$ \\
\hline & 1.47 Sec. & 0 & \\
\hline S8133 & 2.4 Min. & 0 & \\
\hline TE133m & 55.4 Min. & 0 & \\
\hline TE133 & 12.45. Min. & 0 & \\
\hline & & & \\
\hline
\end{tabular}


Table 2.4. (contd)

Table 4-E. Decay $71 m e=40$ Years

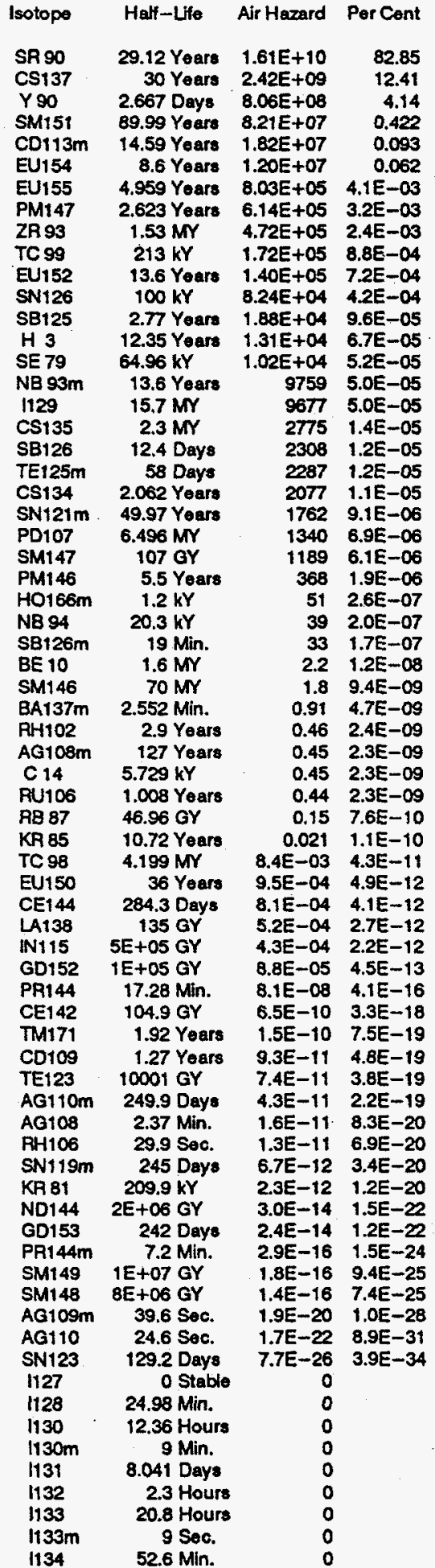

Table 4-F. Decay Time $=100$ Years

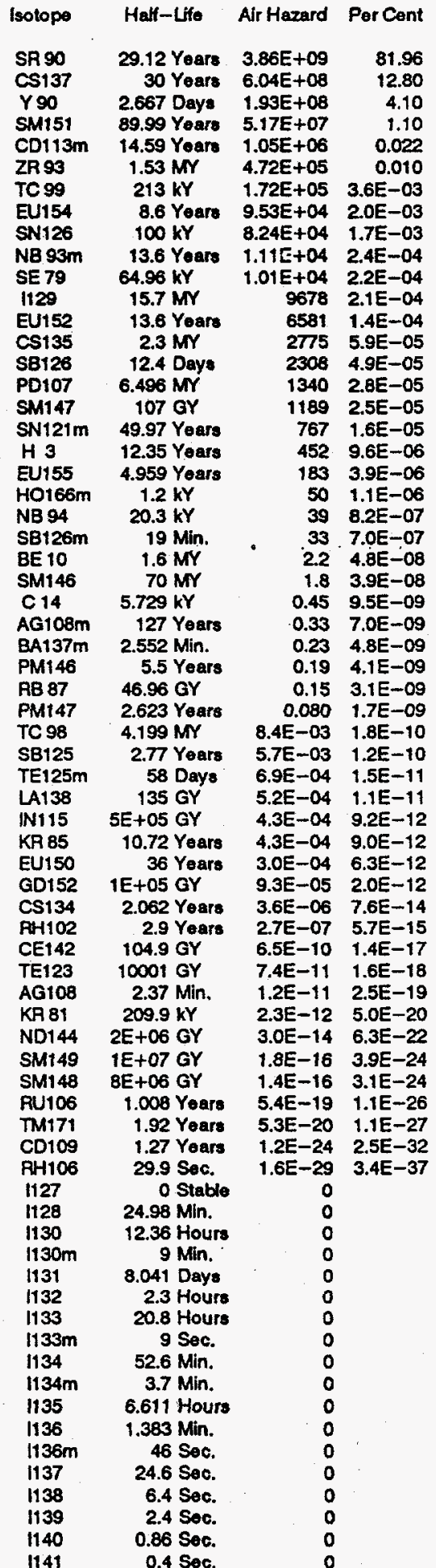


Table 2.4. (contd)

Table 4-O. Decay $7 m e=300$ Years

\begin{tabular}{|c|c|c|c|}
\hline lsotope & Half-Lte & Air Hezard & Per Cent \\
\hline SR 90 & 29.12 Years & 3.31E+07 & 62.98 \\
\hline SMt51 & 89.99 Years & $1.11 E+07$ & 21.10 \\
\hline $\operatorname{cs} 137$ & 30 Years & $5.94 E+06$ & 11.31 \\
\hline$Y 90$ & 2.667 Days & $1.66 E+06$ & 3.15 \\
\hline ZR 93 & $1.53 \mathrm{MY}$ & $4.72 E+05$ & 0.899 \\
\hline TC 99 & $213 \mathrm{kY}$ & $1.72 E+05$ & 0.327 \\
\hline SN126 & $100 \mathrm{kY}$ & $8.23 E+04$ & 0.157 \\
\hline NB 93m & 13.6 Years & $1.12 E+04$ & 0.021 \\
\hline SE 79 & $64.96 \mathrm{kY}$ & $1.01 E+04$ & 0.019 \\
\hline 1129 & $15.7 \mathrm{MY}$ & 9678 & 0.018 \\
\hline CS135 & $2.3 \mathrm{MY}$ & 2775 & 5.3E-03 \\
\hline$S B 126$ & 12.4 Deys & 2305 & 4.4E-03 \\
\hline PO107 & $6.496 \mathrm{MY}$ & 1340 & $2.6 \mathrm{E}-03$ \\
\hline SM147 & 107 & 1189 & 2.3E-03 \\
\hline CD113m & fears & 79 & 1.5E-04 \\
\hline SN121m & 49.97 Years & 48 & 9.1E-05 \\
\hline HO166m & 1.2 & 44 & 8.4E-05 \\
\hline NB 94 & $20.3 \mathrm{kY}$ & 38 & 7.3E-05 \\
\hline SB126m & $19 \mathrm{Min}$. & 33 & 6.3E-05 \\
\hline BE 10 & $1.6 \mathrm{MY}$ & 2.2 & 4.3E-06 \\
\hline SM146 & $70 \mathrm{MY}$ & 1.8 & $3.5 E-\infty 6$ \\
\hline C 14 & $5.729 \mathrm{kY}$ & 0.44 & $8.3 E-07$ \\
\hline EU152 & 13.6 Years & 0.25 & 4.7E- 07 \\
\hline RB 87 & $46.96 \mathrm{GY}$ & 0.15 & $2.8 \mathrm{E}-07$ \\
\hline AG108m & 127 Yoars & 0.11 & 2.1E-07 \\
\hline EU154 & 8.6 Years & 0.010 & $1.8 E-08$ \\
\hline TC 98 & $4.199 \mathrm{MY}$ & $8.4 E-03$ & $1.6 E-08$ \\
\hline H 3 & Years & $6.0 E-03$ & $1.1 E-08$ \\
\hline BA137m & 2.552 Min. & $2.2 E-03$ & 4.3E-09 \\
\hline LA138 & $135 \mathrm{GY}$ & $5.2 E-04$ & $1.0 E-09$ \\
\hline IN115 & $5 E+05 G Y$ & $4.3 E-04$ & $8.3 E-10$ \\
\hline GD152 & $1 E+05 \mathrm{GY}$ & 9.3E-05 & $1.8 E-10$ \\
\hline EU150 & 36 Years & $6.3 E-06$ & $1.2 E-11$ \\
\hline KR 85 & 10.72 Years & $1.0 E-09$ & $2.0 E-15$ \\
\hline CE142 & $104.9 \mathrm{GY}$ & $6.5 E-10$ & $1.2 E-15$ \\
\hline EU155 & 4.959 Years & $1.3 E-10$ & $2.5 E-16$ \\
\hline TE123 & $10001 \mathrm{GY}$ & $7.4 E-11$ & $1.4 E-16$ \\
\hline AG108 & 2.37 Min. & $3.9 E-12$ & $7.5 E-18$ \\
\hline KR 81 & $209.9 \mathrm{kY}$ & $2.3 E-12$ & $4.4 E-18$ \\
\hline PM146 & 5.5 Years & $2.2 E-12$ & $4.1 E-18$ \\
\hline ND144 & $2 E+06 \mathrm{GY}$ & $3.0 E-14$ & $5.7 E-20$ \\
\hline SM149 & $1 E+07 \mathrm{GY}$ & $1.8 E-16$ & $3.5 E-22$ \\
\hline SM148 & $8 \mathrm{E}+06 \mathrm{GY}$ & $1.4 E-16$ & $2.8 E-22$ \\
\hline $\mathrm{RH} 102$ & 2.9 Years & $4.7 E-28$ & $9.0 E-34$ \\
\hline BA133 & 10.74 Years & 0 & \\
\hline SN134 & 0.845 Sec. & 0 & \\
\hline IN134 & 0.078 Sec. & 0 & \\
\hline XE133m & 2.19 Days & 0 & \\
\hline SN132 & 40 Sec. & 0 & \\
\hline SB134 & t1 Sec. & 0 & \\
\hline SB132 & 2.8 Min. & 0 & \\
\hline TE131 & lin. & 0 & \\
\hline IN132 & 0.12 Sec. & 0 & \\
\hline $\mathrm{SB134m}$ & 10. & 0 & \\
\hline TE134 & 41.8 & 0 & \\
\hline CD132 & 0.145 Sec. & 0 & \\
\hline SB131 & 23 Min. & 0 & \\
\hline CS133 & O Stable & 0 & \\
\hline XE132 & iable & 0 & \\
\hline TE131m & 1.25 Days & 0 & \\
\hline $3132 m$ & & 0 & \\
\hline XE131m & 11.9 Days & 0 & \\
\hline 1131 & 8.041 Days & 0 & \\
\hline CS132 & 6.475 Deys & 0 & \\
\hline XE131 & 0 Stable & 0 & \\
\hline TE132 & 3.258 Deys & 0 & \\
\hline BA132 & lable & 0 & \\
\hline XE133 & 5.245 Days & 0 & \\
\hline IN133 & 0.114 & 0 & \\
\hline $1133 \mathrm{~m}$ & c. & 0 & \\
\hline
\end{tabular}

Table 4-H. Dacay Time $=1000$ Years

\begin{tabular}{|c|c|c|c|}
\hline topo & Half-Lifo & Air Hazard & Per Cent \\
\hline $\begin{array}{l}\text { ZR } 93 \\
\text { TC } 99\end{array}$ & $1.53 \mathrm{Mr}$ & $4.72 E+05$ & 57.96 \\
\hline SN126 & $100 \mathrm{kY}$ & $8.19 E+04$ & $\begin{array}{l}2.006 \\
10.06\end{array}$ \\
\hline SM151 & 89.99 Yoars & $5.05 E+04$ & 6.21 \\
\hline NB 93m & 13.6 Yoars & $1.12 E+04$ & 1.38 \\
\hline SE 79 & $64.96 \mathrm{kY}$ & $1.01 E+04$ & 1.23 \\
\hline 1129 & $15.7 \mathrm{Mr}$. & 9678 & 1.19 \\
\hline CS135 & $2.3 \mathrm{MV}$ & 2774 & 0.341 \\
\hline SB126 & 12.4 Days & 2293 & 0.282 \\
\hline PD107 & $6.496 \mathrm{MY}$ & 1340 & 0.165 \\
\hline SM147 & $107 \mathrm{GY}$ & 1189 & 0.146 \\
\hline NB 94 & $20.3 \mathrm{kY}$ & 38 & $4.6 E-03$ \\
\hline $\mathrm{SB} 126 \mathrm{~m}$ & $19 \mathrm{Mtn}$. & 33 & $4.0 E-03$ \\
\hline HO166m & $1.2 \mathrm{kY}$ & 30 & $3.6 E-03$ \\
\hline $\begin{array}{l}\text { BE } 10 \\
\text { SR90 }\end{array}$ & $\begin{array}{c}1.6 \mathrm{Mr} \\
29.12 \text { Yoars }\end{array}$ & $\begin{array}{l}2.2 \\
1.9\end{array}$ & $\begin{array}{l}2.8 \mathrm{E}-04 \\
2.4 \mathrm{E}-0.4\end{array}$ \\
\hline SM146 & 29.12 Toans & $\begin{array}{l}1.9 \\
1.8\end{array}$ & $\begin{array}{l}2.4 E-04 \\
2.3 E-04\end{array}$ \\
\hline CS137 & 30 Years & 0.56 & $6.9 E-05$ \\
\hline$C_{14}$ & $5.729 k$ & 0.40 & $4.9 E-05$ \\
\hline RB 87 & $46.96 \mathrm{G}$ & 0.15 & $1.8 \mathrm{E}-05$ \\
\hline $\begin{array}{l}\text { Y } 90 \\
\text { TC98 }\end{array}$ & 2.667 Days & 0.096 & 1.2E-05 \\
\hline $\begin{array}{l}\text { TC98 } \\
\text { SN121m }\end{array}$ & $\begin{array}{l}4.199 \mathrm{Mr} \\
49.97 \text { Yoars }\end{array}$ & $\begin{array}{l}8.4 E-03 \\
2.9 E-03\end{array}$ & $\begin{array}{l}1.0 E-06 \\
3.6 E-07\end{array}$ \\
\hline AG108m & 127 Years & $2.4 E-03$ & $3.0 E-07$ \\
\hline LA138 & $135 \mathrm{GY}$ & $5.2 E-04$ & $6.4 \mathrm{E}-08$ \\
\hline IN115 & $5 E+05 \mathrm{GY}$ & $4.3 E-04$ & 5.3E-08 \\
\hline G0152 & $1 E+05 \mathrm{GY}$ & 9.3E-05 & $1.1 E-08$ \\
\hline CE142 & 104.9 GY & $6.5 E-10$ & $8.0 E-14$ \\
\hline BA137m & 2.552 Min. & $2.1 E-10$ & $2.6 E-14$ \\
\hline TE123 & $10001 \mathrm{GY}$ & $7.4 E-11$ & $9.1 E-15$ \\
\hline EU150 & 36 Years & $8.9 E-12$ & $1.1 E-15$ \\
\hline KA 81 & $209.9 \mathrm{kY}$ & $2.3 E-12$ & $2.9 E-16$ \\
\hline CD113m & 14.59 Years & $2.8 E-13$ & $3.5 E-17$ \\
\hline AG108 & 2.37 Min. & $8.6 E-14$ & 1.1E-17 \\
\hline ND144 & $2 E+06$ GY & $3.0 E-14$ & $3.7 E-18$ \\
\hline SM149 & $T E+07 \mathrm{GY}$ & $1.8 E-16$ & $2.2 E-20$ \\
\hline SM148 & $8 E+06 \mathrm{GY}$ & $1.4 E-16$ & $1.8 \mathrm{E}-20$ \\
\hline EU152 & 13.6 Years & $7.9 E-17$ & $9.7 \bar{E}-21$ \\
\hline H 3 & 12.35 Years & $5.2 E-20$ & $6.4 \mathrm{E}-24$ \\
\hline KR 85 & 10.72 Years & $2.3 E-29$ & $2.8 E-33$ \\
\hline SB131 & $23 \mathrm{Min}$. & 0 & \\
\hline XE133n & 2.19 Days & 0 & \\
\hline CS133 & O Stablo & 0 & \\
\hline XE133 & 5.245 Days & 0 & \\
\hline $1133 m$ & $9 \mathrm{~S}$ & 0 & \\
\hline BA133 & $10.74 Y$ & 0 & \\
\hline IN134 & $0.078 \mathrm{Se}$ & 0 & \\
\hline SN1 & $0.845 \mathrm{~S}$ & 0 & \\
\hline & 0 & 0 & \\
\hline TE134 & $41.8 \mathrm{M}$ & 0 & \\
\hline SN131 & $1.05 \mathrm{M}$ & & \\
\hline SB134m & 10 & 0 & \\
\hline TE131 & 1.25 Days & 0 & \\
\hline SB13 & $28 M$ & 0 & \\
\hline TE1 & 3. & 0 & \\
\hline 1131 & 8.041 Days & 0 & \\
\hline XE131 & $0 \mathrm{~s}$ & 0 & \\
\hline XE131m & $11.9 \mathrm{D}$ & 0 & \\
\hline CD13 & $0.145 \mathrm{St}$ & 0 & \\
\hline TE13 & $25 \mathrm{M}$ & 0 & \\
\hline IN13 & $0.12 \mathrm{~s}$ & 0 & \\
\hline SN13 & $40 \mathrm{~s}$ & 0 & \\
\hline 1133 & 20.8 Hours & 0 & \\
\hline SB132m & $4.2 \mathrm{Mi}$ & 0 & \\
\hline TE133r & 55.4 Mir & 0 & \\
\hline 1132 & $2.3 \mathrm{Hou}$ & 0 & \\
\hline XE132 & & 0 & \\
\hline BA132 & os & 0 & \\
\hline & $0.114 \mathrm{~S}$ & 0 & \\
\hline SN133 & 170 & 0 & \\
\hline
\end{tabular}


Table 2.4. (contd)

Table $4-1$. Decay Time $=3000$ Years

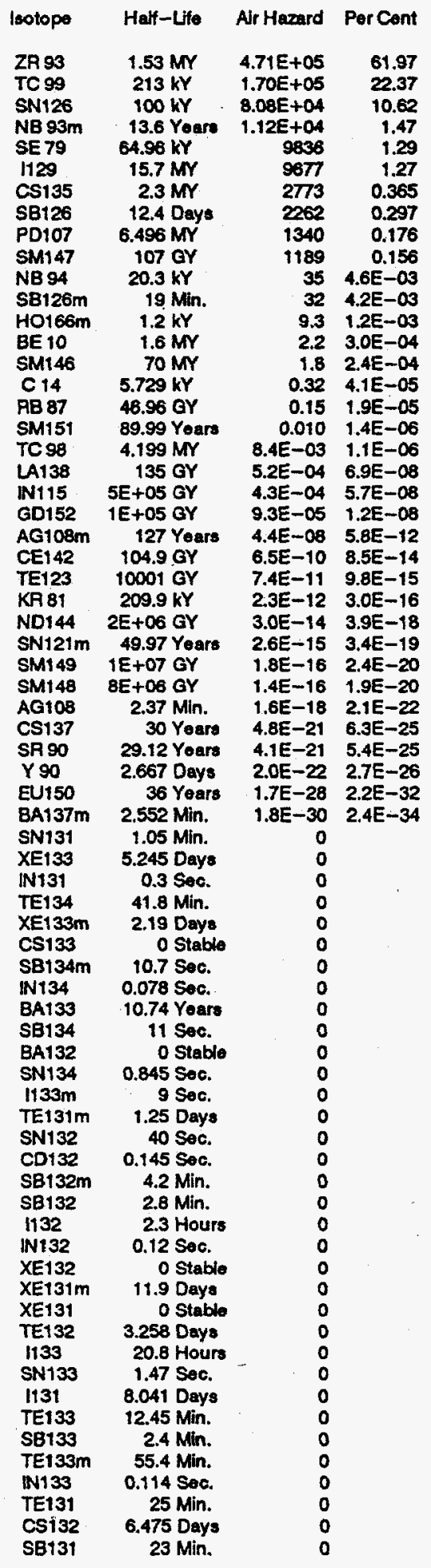


Table 2.5. Relative Inhalation Hazard of Activation Products in Hanford Reactor Fuel and Cladding at Various Decay Times

Table 5-A. Decay Time $=1$ Year

\begin{tabular}{|c|c|c|c|}
\hline & Heff-Lilo & Ar Hazard & en \\
\hline & 5.270 Yoars & 3.31E+07 & 35.68 \\
\hline & & $1 E+06$ & 12.46 \\
\hline & 5.15 Days & $4.80 E+05$ & 8.07 \\
\hline SN119m & 245 Days & $3.21 E+05$ & 5.40 \\
\hline & & & 4.48 \\
\hline & & & 3.52 \\
\hline & 70.78 Days & $4 E+05$ & 3.25 \\
\hline & 115.1 Days & & 1.87 \\
\hline 1123 & 129.2 Days & +05 & 1.53 \\
\hline & Years & & 1.24 \\
\hline & Days & +05 & 1.06 \\
\hline & 92 Years & +05 & 0.637 \\
\hline TE125m & 58 Days & +05 & 0.425 \\
\hline Y91 & lays & +04 & \\
\hline & ays & & \\
\hline & ars & & \\
\hline & & & \\
\hline & $\begin{array}{l}\text { ays } \\
\text { ays }\end{array}$ & & \\
\hline & & $\begin{array}{l}+04 \\
+04\end{array}$ & \\
\hline & 18 & 9901 & 0.017 \\
\hline & & 5348 & $9.0 E-03$ \\
\hline & urs & 3719 & -03 \\
\hline & & 2784 & -03 \\
\hline & & 2189 & -03 \\
\hline & & 1961 & -03 \\
\hline & & 6 . & -03 \\
\hline Si & 49 & 1328 & -03 \\
\hline$N I$ & & 921 & -03 \\
\hline$W$ & & 817 & -03 \\
\hline $\mathrm{CR}$ & 27 & 514 & -04 \\
\hline & & 299 & -04 \\
\hline & & 170 & -04 \\
\hline & & 73 & -04 \\
\hline & & 53 & 8.8 \\
\hline & 12 & 24 & 4.08 \\
\hline & 1.6 & 9.0 & -05 \\
\hline & ars & 4.9 & -06 \\
\hline TE & ys & 4.7 & -06 \\
\hline NF & ars & 2.9 & -06 \\
\hline & & 1.9 & -06 \\
\hline Om & ys & 0.60 & -06 \\
\hline 17 & ys & 0.49 & -07 \\
\hline & 2.6 & 0.24 & -07 \\
\hline $7 \mathrm{~m}$ & & 0.18 & -07 \\
\hline & 3.4 & .14 & -07 \\
\hline 82 & $9 \AA$ & 0.13 & -07 \\
\hline & 2 & 0.057 & -08 \\
\hline ( & Days & 047 & -08 \\
\hline re & 2 & 0.032 & -08 \\
\hline & 1 & 0.021 & -08 \\
\hline & ars & 0.018 & $E-08$ \\
\hline if & ars & 0.0017 & $E-09$ \\
\hline & 6.7 & 0.0016 & $=-09$ \\
\hline & urs & 031 & $5.2 E-10$ \\
\hline P 32 & 1 & 026 & $4.3 E-10$ \\
\hline SN125 & iys & 010 & $1.7 E-10$ \\
\hline & 4 Days & $E-05$ & $6.1 E-11$ \\
\hline Dos & 39.28 Days & $2.8 E-05$ & $4.8 E-18$ \\
\hline B205 & $30 \mathrm{~N}$ & $2.7 E-05$ & $4.5 E-11$ \\
\hline 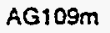 & 39.65 & $E-06$ & $4.8 E-12$ \\
\hline & 74.02 Days & $1.1 E-06$ & $1.9 E-12$ \\
\hline & 30 & $5.9 E-07$ & 9.9 \\
\hline & 5 & $=-07$ & 7.4 \\
\hline & 1.15 & $=-07$ & -13 \\
\hline & 8 & -08 & -14 \\
\hline & 3 & -08 & 2 \\
\hline & $4.159 \mathrm{n}$ & $E-09$ & 3. \\
\hline & $241 Y$ & & \\
\hline & $=0$ & $4.7 E-11$ & -17 \\
\hline
\end{tabular}

Table 5-B. Decay Time $=3$ Yoars

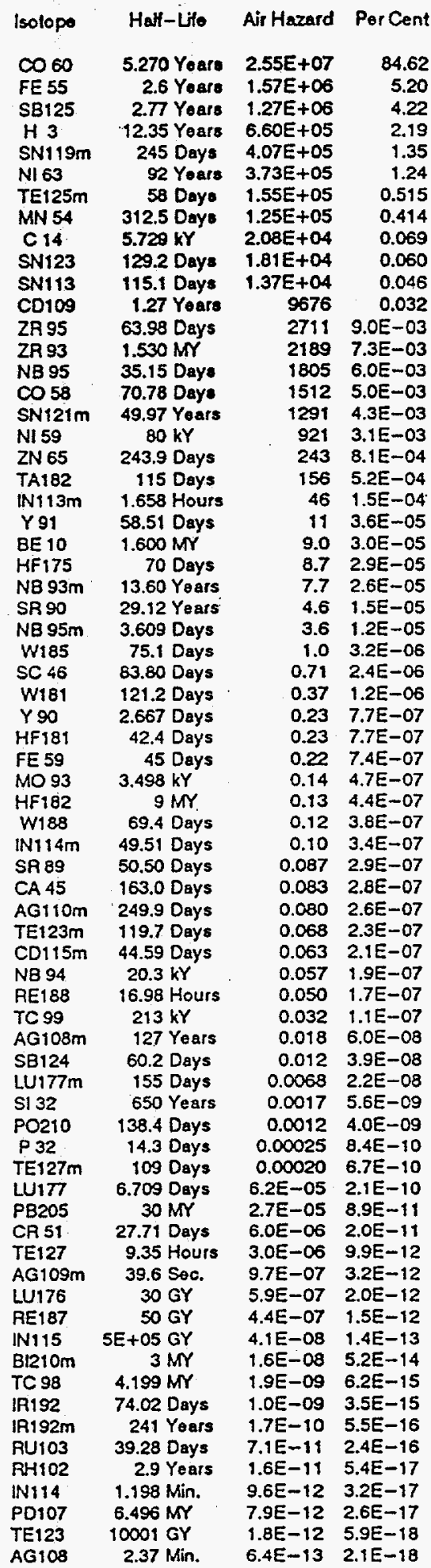


Table 2.5. (contd)

Table 5-C. Decay Time $=10$ Yeare

\begin{tabular}{|c|c|c|c|}
\hline Isotope & Halt-Lito & Air Hazard & Per Cont \\
\hline $\begin{array}{l}\mathrm{CO} 60 \\
\mathrm{H} 3\end{array}$ & $\begin{array}{l}5.270 \text { Years } \\
12.35 \text { Yoers }\end{array}$ & $\begin{array}{l}1.01 E+07 \\
4.46 E+05\end{array}$ & $\begin{array}{r}88.52 \\
3.89\end{array}$ \\
\hline Ni 63 & 92 Years & $3.54 E+05$ & 3.09 \\
\hline FE 55 & 2.6 Years & $2.42 E+05$ & 2.11 \\
\hline SB125 & 2.77 Years & $2.20 E+05$ & 1.92 \\
\hline TE125m & 58 Days & $2.69 E+04$ & 0.234 \\
\hline C 14 & $5.729 \mathrm{kY}$ & $2.08 E+04$ & 0.181 \\
\hline ZR 93 & $1.530 \mathrm{Mr}$ & 2189. & 0.019 \\
\hline SN121m & 49.97 Years & 1172 & 0.010 \\
\hline$N 159$ & $80 \mathrm{kY}$ & 921 & 8.0E-03 \\
\hline MN 54 & 312.5 Days & 429 & $3.7 E-03$ \\
\hline SN119m & 245 Days & 294 & $2.6 E-03$ \\
\hline CD109 & 1.27 Years & 212 & $1.9 E-03$ \\
\hline NB 93m & 13.60 Years & 21 & $1.8 E-04$ \\
\hline BE 10 & $1.600 \mathrm{MN}$ & 9.0 & $7.9 E-05$ \\
\hline SR 90 & 29.12 Years & 3.9 & $3.4 E-05$ \\
\hline$Y 90$ & 2.667 Days & 0.20 & 1.7E-06 \\
\hline $\mathrm{ZN} 65$ & 243.9 Days & 0.17 & $1.5 E-06$ \\
\hline MO 93 & $3.498 \mathrm{kY}$ & 0.14 & $1.2 E-06$ \\
\hline HF182 & $9 \mathrm{MY}$ & 0.13 & $1.2 E-06$ \\
\hline NB 94 & $20.3 \mathrm{kY}$ & 0.057 & $4.9 E-07$ \\
\hline TC 99 & $213 \mathrm{kY}$ & 0.032 & $2.8 E-07$ \\
\hline SN123 & 129.2 Days & 0.020 & $1.7 \varepsilon-07$ \\
\hline$A G 10 B m$ & 127 Years & 0.017 & $1.5 E-07$ \\
\hline SN113 & 115.1 Days & 0.0028 & 2.5E-OB \\
\hline TA182 & 115 Days & 0.0018 & $1.6 E-08$ \\
\hline S1 32 & 650 Years & 0.0017 & $1.5 E-08$ \\
\hline P 32 & 14.3 Days & 0.00025 & $2.2 E-09$ \\
\hline AG110m & 249.9 Days & $6.6 E-05$ & $5.8 E-10$ \\
\hline PB205 & $30 \mathrm{MY}$ & $2.7 E-05$ & $2.3 E-10$ \\
\hline IN113m & 1.658 Hours & $9.4 E-06$ & $8.2 E-11$ \\
\hline$C A 45$ & 163.0 Days & $1.6 E-\infty 6$ & $1.4 E-11$ \\
\hline LU176 & $30 \mathrm{GY}$ & $5.9 E-07$ & $5.1 E-12$ \\
\hline RE 187 & $50 \mathrm{GY}$ & $4.4 E-07$ & $3.8 E-12$ \\
\hline Wisi & 121.2 Days & $1.6 E-07$ & $1.4 E-12$ \\
\hline LU177m & 155 Days & 7.3E-OB & $6.4 E-13$ \\
\hline N115 & $5 E+05 G Y$ & $4.1 E-08$ & $3.6 \mathrm{E}-13$ \\
\hline TE123m & 119.7 Days & $2.5 E-08$ & $2.2 E-13$ \\
\hline$A G 109 m$ & 39.6 Sec. & $2.1 E-08$ & $1.9 E-13$ \\
\hline $\mathrm{CO} 58$ & 70.78 Days & $2.0 E-08$ & $1.8 E-13$ \\
\hline $\mathrm{Bl} 210 \mathrm{~m}$ & $3 \mathrm{Mr}$ & 1.6E-08 & $1.4 E-13$ \\
\hline PO210 & 138.4 Days & $3.5 E-09$ & $3.0 E-14$ \\
\hline ZR 95 & 63.98 Days & $2.5 E-09$ & $2.2 E-14$ \\
\hline TC 98 & $4.199 \mathrm{Mr}$ & $1.9 E-09$ & $1.6 E-14$ \\
\hline NB 95 & 35.15 Days & $1.7 E-09$ & $1.5 E-14$ \\
\hline LU177 & 6.709 Days & $6.7 E-10$ & $5.9 E-15$ \\
\hline $\operatorname{SC~} 46$ & 63.80 Days & $4.6 E-10$ & $4.0 E-15$ \\
\hline IR192m & 241 Years & $1.7 E-10$ & $1.5 E-15$ \\
\hline HF175 & 70 Days & $8.8 E-11$ & $7.7 E-16$ \\
\hline W185 & 75.1 Days & $5.5 E-11$ & $4.8 E-16$ \\
\hline TE $127 \mathrm{~m}$ & 109 Deys & $1.8 E-11$ & $1.5 E-16$ \\
\hline $\mathrm{RH} 102$ & 2.9 Years & $1.6 E-11$ & $1.4 E-16$ \\
\hline IR192 & 74.02 Days & $1.3 E-11$ & $1.2 E-16$ \\
\hline PO107 & $6.496 \mathrm{MY}$ & $7.9 E-12$ & $6.9 E-17$ \\
\hline NB 95m & 3.609 Days & $3.4 E-12$ & $3.0 E-17$ \\
\hline TE123 & $10001 \mathrm{GY}$ & $1.8 E-12$ & $1.6 \mathrm{E}-17$ \\
\hline$W 188$ & 69.4 Days & $9.3 E-13$ & $8.2 E-18$ \\
\hline Y 91 & 58.51 Days & $7.6 E-13$ & $6.6 E-18$ \\
\hline AG108 & 2.37 Min. & $6.1 E-13$ & $5.4 E-18$ \\
\hline RE168 & 16.98 Hours & $4.0 E-13$ & $3.5 E-18$ \\
\hline TE127 & 9.35 Hours & $2.6 E-13$ & $2.2 E-18$ \\
\hline PT193 & 500 Years & $6.6 E-15$ & $5.8 E-20$ \\
\hline SB124 & 60.2 Days & $1.9 E-15$ & $1.7 E-20$ \\
\hline 1129 & $15.7 \mathrm{MY}$ & $1.6 \mathrm{E}-15$ & $1.4 E-20$ \\
\hline$A G 110$ & 24.6 Sec. & $2.6 E-16$ & $2.3 E-21$ \\
\hline RU106 & 1.008 Years & $1.7 E-16$ & $1.4 \mathrm{E}-21$ \\
\hline SR 89 & 50.50 Days & $5.0 E-17$ & $4.3 E-22$ \\
\hline N114m & 49.51 Days & $2.9 E-17$ & $2.5 E-22$ \\
\hline$K 42$ & 12.36 Houts & $6.7 E-18$ & $5.9 E-23$ \\
\hline FE 59 & 45 Days & $1.8 E-18$ & $1.5 E-23$ \\
\hline
\end{tabular}

Table 5-0. Decey Time $=30$ Years

\begin{tabular}{|c|c|c|c|}
\hline Isotope & Hat-Lifo & Air Hezard & Percen \\
\hline $\begin{array}{l}\infty 060 \\
N 163\end{array}$ & $\begin{array}{r}5.270 \text { Years } \\
92 \text { Yoars }\end{array}$ & $\begin{array}{l}7.30 E+05 \\
3.04 E+05\end{array}$ & 25.2 \\
\hline H 3 & $\begin{array}{l}12.35 \text { Years } \\
5.729 \mathrm{kY}\end{array}$ & $\begin{array}{l}1.45 E+05 \\
2.07 E+04\end{array}$ & 12.0 \\
\hline ZR 93 & $1.530 \mathrm{MY}$ & 2188 & 0.18 \\
\hline SB125 & 2.77 & 1476 & 0.12 \\
\hline FE 55 & ears & 1170 & 0.09 \\
\hline NI 59 & & 920 & 0.07 \\
\hline SN121m & 49.97 Years & 888 & 0.07 \\
\hline TE125m & 58 Days & 180 & 0.01 \\
\hline $\mathrm{NB} 93 \mathrm{~m}$ & 13.6 & 41 & $3.4 E-0$ \\
\hline BE 10 & 1.600 & 9.0 & $7.5 E-0$ \\
\hline SA 90 & 29.12 Years & 2.4 & $2.0 E-0$ \\
\hline 93 & $3.498 \mathrm{kY}$ & 0.14 & $1.2 E-05$ \\
\hline 182 & & 0.13 & \\
\hline 90 & 2.667 Deys & 0.12 & 1.OE-0 \\
\hline NB 94 & $20.3 \mathrm{kY}$ & 0.057 & 4.7E-OE \\
\hline & & 0.032 & $2.7 E-06$ \\
\hline AG108m & 127 Years & 0.015 & $1.3 E-06$ \\
\hline CD109 & 1.27 Years & 0.0039 & $3.2 E-07$ \\
\hline TA182 & 115 Days & 0.0018 & $1.5 E-07$ \\
\hline SI 32 & 650 & 0.0016 & $1.4 E-07$ \\
\hline P 32 & 14.3 & 0.00025 & 2.OE-OB \\
\hline MN 54 & 312.5 & -05 & $3.3 E-09$ \\
\hline PB205 & 30 & -05 & $2.2 E-09$ \\
\hline 10 & 30 & -07 & 4.9E- 11 \\
\hline RE187 & $50 \mathrm{GY}$ & $4.4 E-07$ & $3.6 E-11$ \\
\hline SN119m & 245 & $=-07$ & $2.6 E-11$ \\
\hline IN115 & $5 E+05 G Y$ & $4.1 E-08$ & $3.4 E-12$ \\
\hline $\mathrm{Bl} 1210 \mathrm{~m}$ & $3 \mathrm{MY}$ & $1.6 E-08$ & $1.3 E-12$ \\
\hline TC & 4.199 & $E-09$ & $1.5 \mathrm{E}-13$ \\
\hline & 243.9 Deys & $1.6 E-10$ & $1.3 E-14$ \\
\hline IR192m & 241 Years & $1.6 E-10$ & $1.3 E-14$ \\
\hline PO210 & 138.4 Days & $1.2 E-10$ & $1.0 E-14$ \\
\hline $\mathrm{RH} 102$ & 2.9 Years & $1.6 E-11$ & $1.3 E-15$ \\
\hline IR192 & 74.02 Days & $1.3 E-11$ & $1.0 \mathrm{E}-15$ \\
\hline PD107 & $6.496 \mathrm{MY}$ & $7.9 E-12$ & $6.6 \mathrm{E}-16$ \\
\hline TE123 & $10001 \mathrm{GY}$ & $E-12$ & $1.5 E-16$ \\
\hline AG108 & 2.37 Min. & $5.5 E-13$ & $4.6 E-17$ \\
\hline AG109m & 39.6 Sec. & $3.9 E-13$ & $3.2 E-17$ \\
\hline AG110 & 249.9 Days & $1.0 E-13$ & $8.7 E-18$ \\
\hline PT193 & 500 Years & $6.6 E-15$ & $5.5 E-19$ \\
\hline 1129 & $15.7 \mathrm{MY}$ & $1.6 E-15$ & $1.4 E-19$ \\
\hline$K 42$ & 12.36 Hours & $6.7 E-18$ & $5.6 E-22$ \\
\hline SN 123 & 129.2 Days & $1.9 E-19$ & $1.6 \mathrm{E}-23$ \\
\hline$\vee 50$ & $4 E+07 \mathrm{GY}$ & $3.7 E-19$ & $1.4 E-23$ \\
\hline PB204 & $1 E+08 G Y$ & $1.3 E-19$ & $1.1 E-23$ \\
\hline CA 45 & 163.0 Days & $5.1 E-20$ & $4.2 E-24$ \\
\hline B1208 & $368 \mathrm{kY}$ & $4.7 E-20$ & $3.9 E-24$ \\
\hline TL2OS & $4.19 \mathrm{Min}$. & -20 & $2.6 E-24$ \\
\hline TM171 & 1.92 Years & $6.5 E-22$ & $5.4 E-26$ \\
\hline $\mathrm{LU} 177 \mathrm{~m}$ & 155 Dsys & $4.8 E-22$ & $3.9 E-26$ \\
\hline SN113 & 115.1 Days & $2.2 E-22$ & $1.8 E-26$ \\
\hline RU106 & 1.008 Years & $1.8 E-22$ & $1.5 E-26$ \\
\hline LU17 & 6.709 Days & $4.4 E-24$ & $3.6 E-28$ \\
\hline IN113m & 1.658 Hours & $7.4 E-25$ & $6.1 E-29$ \\
\hline$A G: 10$ & $24.6 \mathrm{Sec}$. & $4.2 E-25$ & $3.5 E-29$ \\
\hline W181 & 121.2 Days & $1.2 E-25$ & $9.7 E-30$ \\
\hline A 42 & 33 Years & -26 & $5.6 E-30$ \\
\hline E123m & 119.7 Days & $1.1 E-26$ & $8.8 E-31$ \\
\hline lan & 29.9 Sec. & $5.3 E-33$ & $4.4 E-37$ \\
\hline & & 0 & \\
\hline 105 & 1.196 Days & 0 & \\
\hline & 15 Min. & 0 & \\
\hline & $135 \mathrm{GY}$ & 0 & \\
\hline & o Stable & 0 & \\
\hline & 10.74 Years & 0 & \\
\hline & 1.62 Days & 0 & \\
\hline & $70 \mathrm{Mr}$ & 0 & \\
\hline 4134 & o Stable & 0 & \\
\hline
\end{tabular}


Table 2.5. (contd)

Table 5-E. Decay Time $=40$ Years

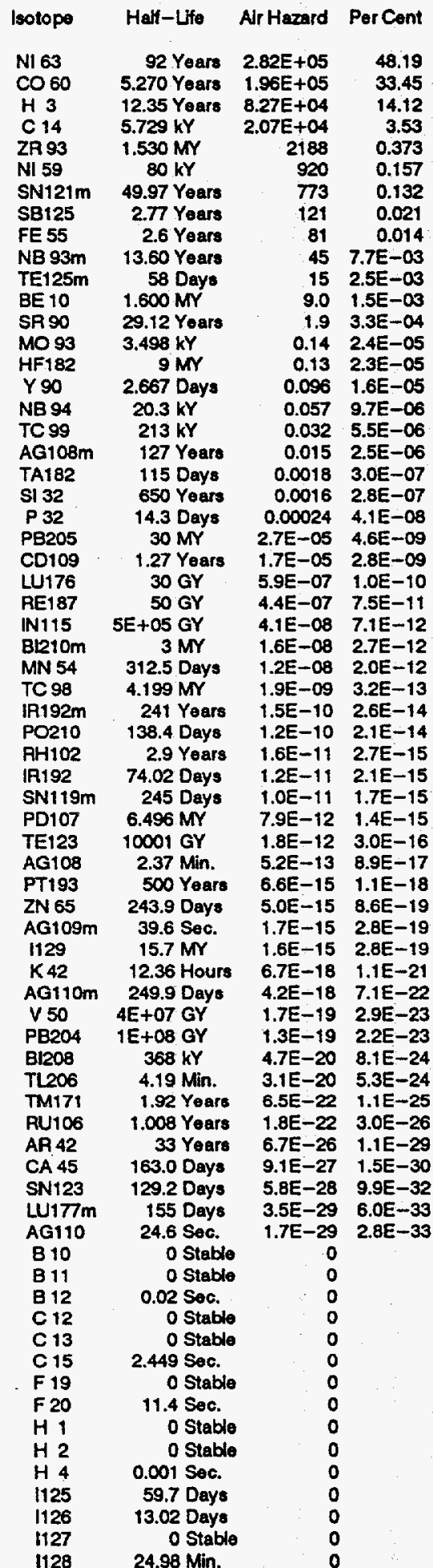

Table 5-F. Decay Time $=100$ Yeare

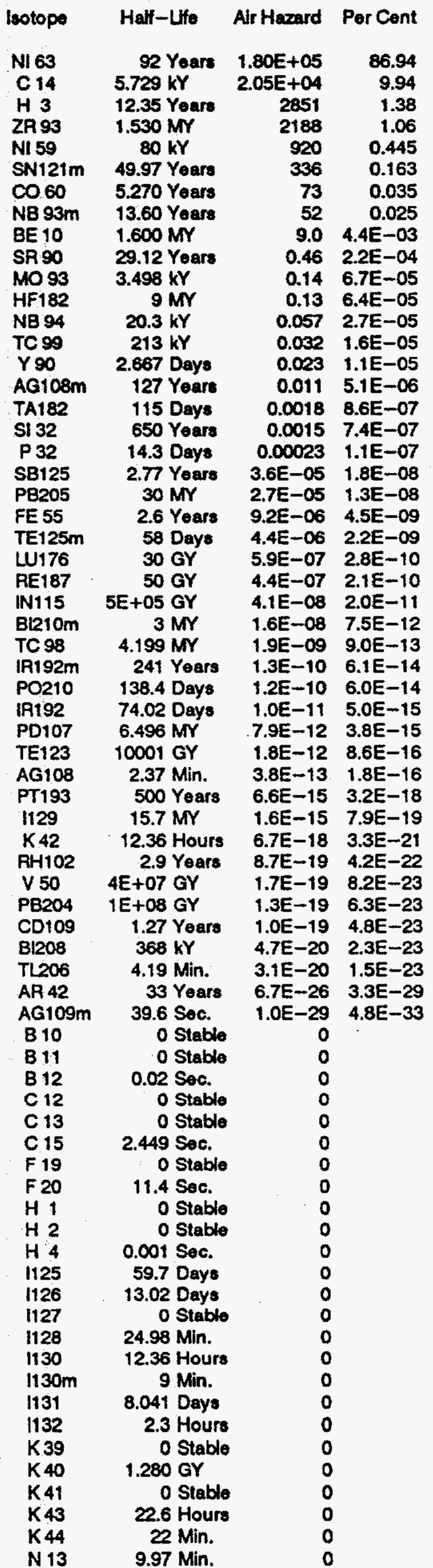


Table 2.5. (contd)

Table 5-G. Docay Time $=300$ Years

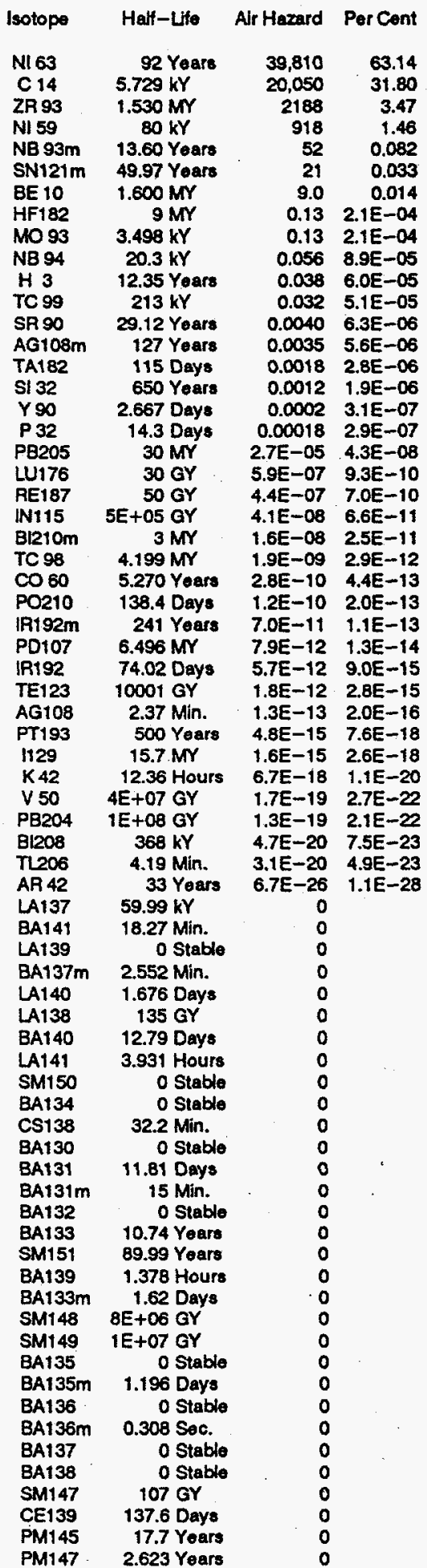

Table 5-H. Decay Time $=1000$ Years

\begin{tabular}{|c|c|c|c|}
\hline otope & Half-Lifo & Air Hazard & PerCon \\
\hline 5.4 & $5.729 \mathrm{kY}$ & 18,420 & 84.55 \\
\hline ZR 93 & $1.530 \mathrm{MN}$ & 2188 & 10.04 \\
\hline NI 59 & $80 \mathrm{kY}$ & 913 & 4.19 \\
\hline NI 63 & 92 Yoars & 204 & 0.936 \\
\hline NB 93m & 13.60 Years & 52 & 0.238 \\
\hline BE 10 & $1.600 \mathrm{Mr}$ & 9.0 & 0.042 \\
\hline HF182 & $9 \mathrm{MN}$ & 0.13 & 6.1E-04 \\
\hline MO 93 & $3.498 \mathrm{kY}$ & 0.12 & 5.3E-04 \\
\hline NB 94 & $20.3 \mathrm{kY}$ & 0.055 & $\begin{array}{l}2.5 E-04 \\
1.5 E-04\end{array}$ \\
\hline $\begin{array}{l}\text { TC } 99 \\
\text { TA182 }\end{array}$ & $\begin{array}{l}213 \mathrm{kY} \\
115 \text { Days }\end{array}$ & $\begin{array}{r}0.032 \\
0.0018\end{array}$ & $\begin{array}{l}1.5 E-04 \\
8.1 E-06\end{array}$ \\
\hline SN121m & 49.97 Years & 0.0013 & $5.8 E-06$ \\
\hline $\begin{array}{l}\text { S1 } 32 \\
\text { P } 32\end{array}$ & $\begin{array}{c}650 \text { Yoars } \\
14.3 \text { Days }\end{array}$ & $\begin{array}{r}0.00058 \\
8.7 E-05\end{array}$ & $\begin{array}{l}2.7 E-06 \\
4.0 E-07\end{array}$ \\
\hline AG10Bm & 127 Years & $7.8 E-05$ & 3.6E-07 \\
\hline PB205 & $30 \mathrm{MY}$ & 2.7E-05 & $1.2 E-07$ \\
\hline Wt76 & $30 \mathrm{GY}$ & $5.9 E-07$ & 2.7E-09 \\
\hline $\begin{array}{l}\text { RE187 } \\
\text { IN115 }\end{array}$ & $\begin{array}{r}50 \mathrm{GY} \\
5 E+05 \mathrm{GY}\end{array}$ & $\begin{array}{l}4.4 E-07 \\
4.1 E-08\end{array}$ & $\begin{array}{l}2.0 E-09 \\
1.9 E-10\end{array}$ \\
\hline B1210m & $3 \mathrm{MY}$ & $1.6 E-08$ & $7.2 E-11$ \\
\hline TC 98 & $4.199 \mathrm{Mr}$ & $1.9 E-09$ & $\begin{array}{l}8.5 E-12 \\
1.1 E-12\end{array}$ \\
\hline $\begin{array}{l}\text { SR 90 } \\
\text { PO210 }\end{array}$ & $\begin{array}{l}29.12 \text { Yoars } \\
138.4 \text { Days }\end{array}$ & $\begin{array}{l}2.3 E-10 \\
1.2 E-10\end{array}$ & $\begin{array}{l}1.1 E-12 \\
5.7 E-13\end{array}$ \\
\hline $\begin{array}{l}\text { PO210 } \\
\text { Y } 90\end{array}$ & $\begin{array}{l}138.4 \text { Days } \\
2.667 \text { Days }\end{array}$ & $\begin{array}{l}1.2 E-10 \\
1.1 E-11\end{array}$ & $5.3 E-14$ \\
\hline IR192m & 241 Years & $1.1 E-11$ & 5.0E-14 \\
\hline PD107 & $6.496 \mathrm{MY}$ & $7.9 E-12$ & $3.6 E-14$ \\
\hline TE123 & $10001 \mathrm{GY}$ & $1.8 E-12$ & $8.2 E-15$ \\
\hline IRI92. & 74.02 Days & $E-13$ & $4.0 E-15$ \\
\hline AG108 & $2.37 \mathrm{Min}$. & 2.8E-15 & 1.3E-17 \\
\hline PT193 & 500 Years & $2.4 E-15$ & $1.1 \mathrm{E}-17$ \\
\hline 1129 & $15.7 \mathrm{MY}$ & $1.6 E-15$ & $7.5 E-18$ \\
\hline H 3 & 12.35 Years & 3.3E-19 & $1.5 E-21$ \\
\hline$\vee 50$ & $4 E+07 \mathrm{GY}$ & $1.7 E-19$ & $7.8 E-22$ \\
\hline PB204 & $1 E+08 \mathrm{GY}$ & $1.3 E-19$ & $6.0 E-22$ \\
\hline B1208 & $368 \mathrm{kY}$ & 4.7E-20 & $2.2 E-22$ \\
\hline$\pi 206$ & $4.19 \mathrm{Min}$. & $3.1 E-20$ & $1.4 E-22$ \\
\hline$K 42$ & 12.36 Hours & $2.8 E-24$ & $1.3 E-26$ \\
\hline & & $2.8 E-32$ & $1.3 E-34$ \\
\hline BA141 & 18.27 Min. & 0 & \\
\hline BA130 & 0 Stable & & \\
\hline LA140 & $1.676 \mathrm{De}$ & & \\
\hline BA140 & 12.79 Days & 0 & \\
\hline LA139 & 0 Stable & 0 & \\
\hline 137 & 30 Yes & 0 & \\
\hline$S M 146$ & $70 \mathrm{Mr}$ & 0 & \\
\hline LA138 & $135 \mathrm{GY}$ & 0 & \\
\hline CS138 & 32.2 Min. & 0 & \\
\hline LA137 & $59.99 \mathrm{kY}$ & 0 & \\
\hline SM148 & $8 E+06 \mathrm{GY}$ & 0 & \\
\hline BA13 & 1.378 Hours & 0 & \\
\hline BA13 & O St & 0 & \\
\hline RAM & O St & & \\
\hline SM144 & o st & 0 & \\
\hline 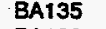 & & 0 & \\
\hline BA13 & $10.74 Y_{0}$ & 0 & \\
\hline SM151 & $89.99 Y_{6}$ & & \\
\hline BA131m & $15 \mathrm{Min}$ & 0 & \\
\hline BA133m & $1.62 \mathrm{Da}$ & 0 & \\
\hline SM15 & 0 Sta & 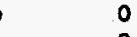 & \\
\hline SM149 & $1 E+07$ GY & 0 & \\
\hline BA13 & & & \\
\hline BA131 & $11.81 \mathrm{D}$ & 0 & \\
\hline BA135m & $1.196 \mathrm{Da}$ & & \\
\hline BA136 & & 0 & \\
\hline CE137m & 1.433 Days & 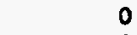 & \\
\hline BA136m & $0.308 \mathrm{Sec}$ & 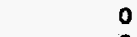 & \\
\hline BA137 & $0 \mathrm{~s}$ & & \\
\hline BA137m & $\mathrm{M}$ & & \\
\hline PM15 & $\mathrm{H}$ & 0 & \\
\hline & & & \\
\hline
\end{tabular}


Table 2.5. (contd)

Table 5-1. Decay Time $=3000$ Years

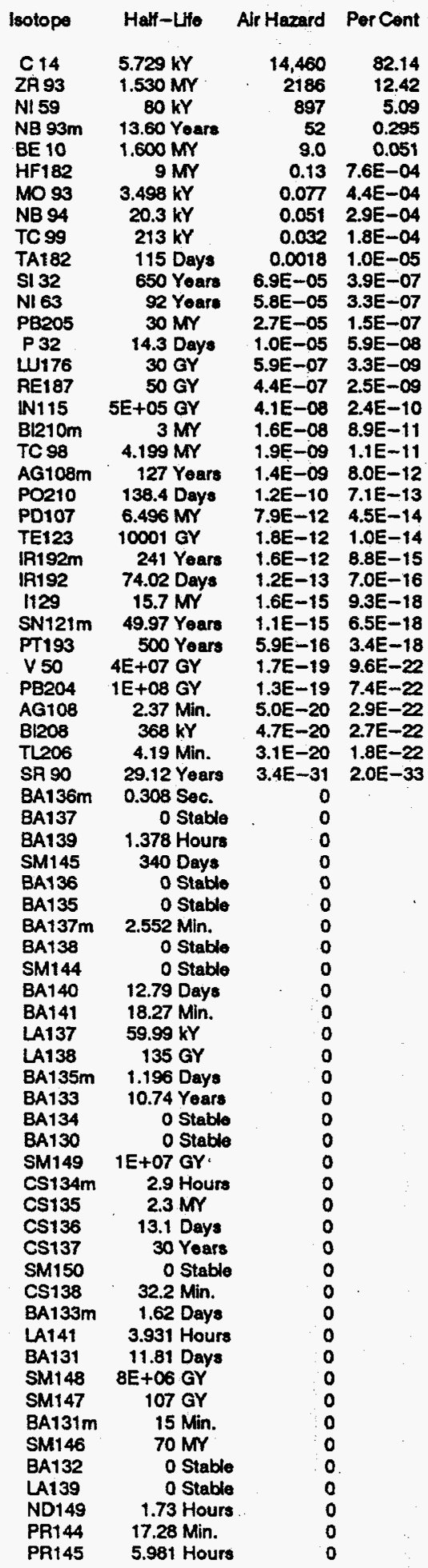


Table 2.6. Relative Inhalation Hazard of Actinides in Hanford Reactor Fuel for Various Decay Times

Table 6-A. Decay Time $=1$ Yoar

Table 6-B. Docay Timo $=3$ Yoars




Table 2.6. (contd)

Table 5-C. Decay Tme $=10$ Yoars

\begin{tabular}{|c|c|c|c|}
\hline Isolopo & Haft-Lifo & Air Hazard & Por Cent \\
\hline $\begin{array}{l}\text { PU239 } \\
\text { PU240 }\end{array}$ & $24.06 \mathrm{kY}$ & $1.22 E+12$ & 63.56 \\
\hline PU240 & $6.537 \mathrm{kY}$ & $2.61 E+11$ & 13.58 \\
\hline AM241 & 432.2 Years & $2.13 E+11$ & 11.08 \\
\hline PU241 & 14.4 Years & $1.56 E+11$ & 8.09 \\
\hline PU238 & 87.74 Years & $6.29 E+10$ & 3.27 \\
\hline U234 & $244.5 \mathrm{kY}$ & $3.88 E+09$ & 0.202 \\
\hline U238 & $4.468 \mathrm{GY}$ & $3.34 \mathrm{E}+09$ & 0.173 \\
\hline NP237 & $2.14 \mathrm{Mr}$ & $2.60 E+08$ & 0.014 \\
\hline$C M 244$ & 18.11 Years & $2.18 E+08$ & 0.011 \\
\hline U235 & $703.8 \mathrm{MY}$ & $1.58 E+08$ & 8.2E-0.3 \\
\hline U236 & $23.41 \mathrm{MY}$ & $9.58 E+07$ & $5.0 E-03$ \\
\hline$A M 242 m$ & 152 Years & 2.22E+07 & $1.2 E-03$ \\
\hline PU242 & $386.9 \mathrm{kY}$ & $1.16 E+07$ & $6.0 E-04$ \\
\hline AM243 & $7.38 \mathrm{kY}$ & $5.25 E+06$ & $2.7 E-0.4$ \\
\hline U232 & 72 Years & $2.14 E+06$ & $1.1 E-04$ \\
\hline TH228 & 1.913 Yoars & $1.00 E+06$ & $5.2 E-05$ \\
\hline$T H 234$ & 24.1 Days & 8.34E+05 & $4.3 E-05$ \\
\hline$C M 242$ & 163.2 Days & $7.84 E+05$ & $4.1 E-05$ \\
\hline TH230 & $\pi \mathrm{kY}$ & $6.42 E+05$ & $3.3 E-05$ \\
\hline $\mathrm{CM} 243$ & 28.5 Years & $6.06 E+05$ & $3.2 E-05$ \\
\hline PU236 & 2.851 Years & $6.00 E+05$ & $3.1 E-05$ \\
\hline PA231 & $32.77 \mathrm{kY}$ & $3.11 E+05$ & $1.6 E-05$ \\
\hline$A C 227$ & 21.77 Years & $4.58 E+04$ & $2.4 E-06$ \\
\hline RA224 & 3.66 Days & 9997 & $5.2 E-07$ \\
\hline U233 & $158.5 \mathrm{kY}$ & 6664 & $3.5 E-07$ \\
\hline PA233 & 27 Days & 5192 & $2.7 E-07$ \\
\hline AM242 & 16.02 Hours & 3316 & $1.7 E-07$ \\
\hline CM245 & $8.499 \mathrm{kY}$ & 2772 & $1.4 E-07$ \\
\hline U237 & 6.75 Days & 1908 & $9.9 E-0 B$ \\
\hline TH231 & 1.063 Days & 1425 & $7.4 E-08$ \\
\hline TH-227 & 18.72 Days & 645 & $3.4 E-08$ \\
\hline PB212 & 10.64 Hours & 500 & $2.6 E-08$ \\
\hline RA223 & 11.43 Days & 229 & $1.2 E-08$ \\
\hline NP236 & $115 \mathrm{kY}$ & 114 & $5.9 E-09$ \\
\hline RA226 & $1.6 \mathrm{kY}$ & 71 & 3.7E-09 \\
\hline TH229 & $7.339 \mathrm{kY}$ & 70 & $3.6 \mathrm{E}-09$ \\
\hline $\mathrm{B} 1212$ & 1.009 Houts & 57 & $3.0 E-09$ \\
\hline NP239 & 2.355 Days & 32 & $1.6 E-09$ \\
\hline $\mathrm{CM} 246$ & $4.731 \mathrm{KY}$ & 23 & $1.2 E-09$ \\
\hline PA234 & 6.7 Hours & 22 & $1.1 E-09$ \\
\hline PN220 & $55.6 \mathrm{Sec}$. & 13 & $6.9 E-10$ \\
\hline NP238 & 2.117 Days & 8.3 & $4.3 E-10$ \\
\hline PB210 & 22.3 Years & 7.7 & $4.0 E-10$ \\
\hline PO210 & 138.4 Days & 6.9 & $3.6 E-10$ \\
\hline $\mathrm{AC225}$ & 10 Days & 0.49 & $2.5 E-11$ \\
\hline THi232 & $14.05 \mathrm{GY}$ & 0.43 & $2.2 E-11$ \\
\hline RA225 & 14.8 Days & 0.24 & $1.3 E-11$ \\
\hline PB211 & $36.1 \mathrm{Min}$. & 0.23 & $1.2 E-11$ \\
\hline$B 1210$ & 5.012 Days & 0.12 & $6.0 E-12$ \\
\hline PU244 & $82.61 \mathrm{Mr}$ & 0.061 & $3.2 E-12$ \\
\hline PB214 & $26.8 \mathrm{Min}$. & 0.035 & $1.8 E-12$ \\
\hline B1214 & 19.9 Min. & 0.035 & $1.8 E-12$ \\
\hline $\mathrm{AN} 222$ & 3.824 Days & 0.024 & $1.2 E-12$ \\
\hline NP235 & 1.084 Years & 0.0070 & $3.7 E-13$ \\
\hline FR223 & 21.8 Min. & 0.0032 & $1.6 E-13$ \\
\hline 81213 & 45.65 Min. & 0.00061 & $3.2 E-14$ \\
\hline RA228 & 6.7 Years & 0.00054 & $2.8 E-14$ \\
\hline PA234m & 1.17 Min. & 0.00033 & $1.7 E-14$ \\
\hline$A C 228$ & 6.131 Hours & $1.6 \mathrm{E}-05$ & $8.5 \bar{E}-16$ \\
\hline $\mathrm{B} 209$ & 3.3 Hours & $4.9 E-\infty 6$ & $2.5 E-16$ \\
\hline CM247 & $15.6 \mathrm{MY}$ & $2.9 E-06$ & $1.5 E-16$ \\
\hline $\mathrm{CM} 248$ & $339.1 \mathrm{kY}$ & $1.2 E-06$ & $6.1 E-17$ \\
\hline U240 & 14.1 Hours & $4.1 E-07$ & $2.1 E-17$ \\
\hline CF249 & 350.5 Years & $2.6 E-07$ & $1.3 E-17$ \\
\hline PO216 & 0.15 Sec. & $4.0 E-08$ & $2.1 E-18$ \\
\hline $\mathrm{PO} 212$ & $3 E-07$ Sec. & $2.6 E-08$ & $1.3 E-18$ \\
\hline CF250 & 13.08 Years & $1.6 E-08$ & $8.4 E-19$ \\
\hline$\pi 208$ & $3.07 \mathrm{Min}$. & $1.4 E-08$ & $7.5 E-19$ \\
\hline & 320 Days & $1.7 E-09$ & 8. $8 E-20$ \\
\hline RN219 & $3.96 \mathrm{Sec}$. & $4.6 E-10$ & $2.4 E-20$ \\
\hline
\end{tabular}

Tablo 6-D. Decay Time $=30$ Yoars

\begin{tabular}{|c|c|c|c|}
\hline Isolopo & Hatt-Lito & Air Hazard & Por Cent \\
\hline $\begin{array}{l}\text { PU239 } \\
\text { AM241 }\end{array}$ & $\begin{array}{l}24.06 \mathrm{kY} \\
432.2 \text { Years }\end{array}$ & $\begin{array}{l}1.22 E+12 \\
4.16 E+11\end{array}$ & $\begin{array}{l}60.49 \\
20.61\end{array}$ \\
\hline PU240 & $6.537 \mathrm{kY}$ & $2.60 E+11$ & 12.90 \\
\hline PU241 & 14.4 Yoare & $5.94 E+10$ & 2.94 \\
\hline PU238 & 87.74 Yoars & $5.37 E+10$ & 2.66 \\
\hline U234 & $244.5 \mathrm{kY}$ & $3.88 E+09$ & 0.192 \\
\hline U238 & $4.468 \mathrm{GY}$ & $3.34 E+09$ & 0.165 \\
\hline NP237 & $2.14 M Y$ & $2.63 E+08$ & 0.013 \\
\hline U235 & $703.8 \mathrm{MY}$ & $1.58 E+08$ & $7.8 E-03$ \\
\hline CM244 & 18.11 Yoars & $1.02 E+08$ & $5.0 \mathrm{E}-03$ \\
\hline U236 & $23.41 \mathrm{Mr}$ & $9.58 E+07$ & 4.7E-03 \\
\hline A $14242 m$ & 152 Yoare & $2.03 E+07$ & $1.0 E-03$ \\
\hline PU242 & $386.9 \mathrm{kY}$ & $1.16 E+07$ & $5.7 E-04$ \\
\hline AM243 & $7.38 \mathrm{kY}$ & $5.24 E+\infty 6$ & $2.6 E-04$ \\
\hline TH230 & $\pi \mathrm{kY}$ & $1.90 E+\infty 6$ & $9.4 E-05$ \\
\hline U232 & 72 Yoars & $1.87 E+06$ & 9.3E-05 \\
\hline TH228 & 1.913 Yoars & $9.59 E+05$ & $4.8 E-05$ \\
\hline PA231 & $32.77 \mathrm{kY}$ & $9.13 E+05$ & $4.5 E-05$ \\
\hline TH234 & 24.1 Days & $8.34 E+05$ & $4.1 E-05$ \\
\hline CM242 & 163.2 Days & $7.15 E+05$ & $3.5 E-05$ \\
\hline $\mathrm{CM} 243$ & 28.5 Years & $3.73 E+05$ & $1.8 \mathrm{E}-05$ \\
\hline AC227 & 21.77 Years & $3.27 E+05$ & $1.6 E-05$ \\
\hline U233 & $158.5 \mathrm{kY}$ & $1.17 E+04$ & $5.8 E-07$ \\
\hline RA224 & 3.66 Days & 9591 & $4.8 \mathrm{E}-07$ \\
\hline PA233 & 27 Days & 5257 & $2.6 E-07$ \\
\hline PU236 & 2.851 Years & 4652 & $2.3 E-07$ \\
\hline TH227 & 18.72 Days & 4613 & $2.3 E-07$ \\
\hline AM242 & 16.02 Hours & 3027 & 1.5E-07 \\
\hline CM245 & $8.499 \mathrm{kY}$ & 2758 & $1.4 E-07$ \\
\hline RA223 & 11.43 Days & 1637 & $8.1 E-08$ \\
\hline$T H 231$ & 1.063 Days & 1425 & $7.1 E-08$ \\
\hline U237 & 6.75 Days & 729 & $3.6 E-08$ \\
\hline PA226 & $1.6 \mathrm{kY}$ & 619 & $3.1 E-08$ \\
\hline PB212 & 10.64 Hours & 480 & $2.4 E-08$ \\
\hline TH1229 & $7.339 \mathrm{kY}$ & 293 & 1.5E-08 \\
\hline PB210 & 22.3 Years & 173 & $8.6 E-09$ \\
\hline PO210 & 138.4 Days & 156 & 7.7E-09 \\
\hline NP236 & $115 \mathrm{kY}$ & 114 & $5.6 \mathrm{E}-09$ \\
\hline B1212 & 1.009 Hours & 55 & 2.7E-09 \\
\hline NP239 & 2.355 Days & 31 & $1.6 \mathrm{E}-09$ \\
\hline CM246 & $4.731 \mathrm{kY}$ & 23 & $1.1 E-09$ \\
\hline PA234 & 6.7 Hours & 22 & $1.1 E-09$ \\
\hline RN220 & $55.6 \mathrm{Sec}$. & 13 & $6.3 E-10$ \\
\hline NP238 & 2.117 Days & 7.6 & $3.8 E-10$ \\
\hline B1210 & 5.012 Days & 2.6 & $1.3 E-10$ \\
\hline AC225 & 10 Days & 2.1 & $1.0 E-10$ \\
\hline PB211 & 36.1 Min. & 1.6 & $8.1 E-11$ \\
\hline TH232 & $14.05 \mathrm{GY}$ & 1.3 & $6.3 E-11$ \\
\hline PA225 & 14.8 Days & 1.0 & $5.1 E-11$ \\
\hline B1214 & 19.9 Min. & 0.31 & $1.5 \mathrm{E}-11$ \\
\hline PB214 & 25.8 Min. & 0.31 & $1.5 E-11$ \\
\hline & 3.824 Oays & 0.21 & $1.0 E-11$ \\
\hline PU244 & $82.61 \mathrm{MY}$ & 0.061 & $3.0 E-12$ \\
\hline FPPOP & 21.8 Min. & 0.023 & $1.1 E-12$ \\
\hline RAPB & 6.7 Years & 0.0030 & $1.5 E-13$ \\
\hline 31213 & 45.65 Min. & 0.0026 & $1.3 E-13$ \\
\hline PA234m & 1.17 Min. & 0.00033 & $1.7 E-14$ \\
\hline & 6.131 Hours & $8.9 E-05$ & $4.4 E-15$ \\
\hline PB20S & 3.3 Hours & $2.1 E-05$ & $1.0 E-15$ \\
\hline bets & $15.6 \mathrm{Mr}$ & $2.9 E-06$ & $1.4 E-16$ \\
\hline $\mathrm{CM}_{24}$ & $339.1 \mathrm{kY}$ & $1.2 E-06$ & $5.8 E-17$ \\
\hline U240 & 14.1 Hours & $4.1 E-07$ & $2.0 E-17$ \\
\hline CF249 & 350.5 Years & $2.5 E-07$ & $1.2 E-17$ \\
\hline 0216 & $0.15 \mathrm{Sec}$. & $3.8 E-08$ & $1.9 \mathrm{E}-18$ \\
\hline & 3E-07 Sec. & $2.5 E-08$ & $1.2 E-18$ \\
\hline PP & 1.084 Years & $2.0 E-08$ & $9.8 E-19$ \\
\hline$F 250$ & 13.08 Years & $1.6 E-08$ & $8.0 E-19$ \\
\hline$\Gamma 2$ & $3.07 \mathrm{Min}$. & $1.4 E-08$ & $6.8 E-19$ \\
\hline & 3.96 Sec. & $3.3 E-09$ & $1.6 \mathrm{E}-19$ \\
\hline & & 3.3E- & \\
\hline
\end{tabular}


Table 2.6. (contd)

Table 6-E. Decay Time $=40$ Years

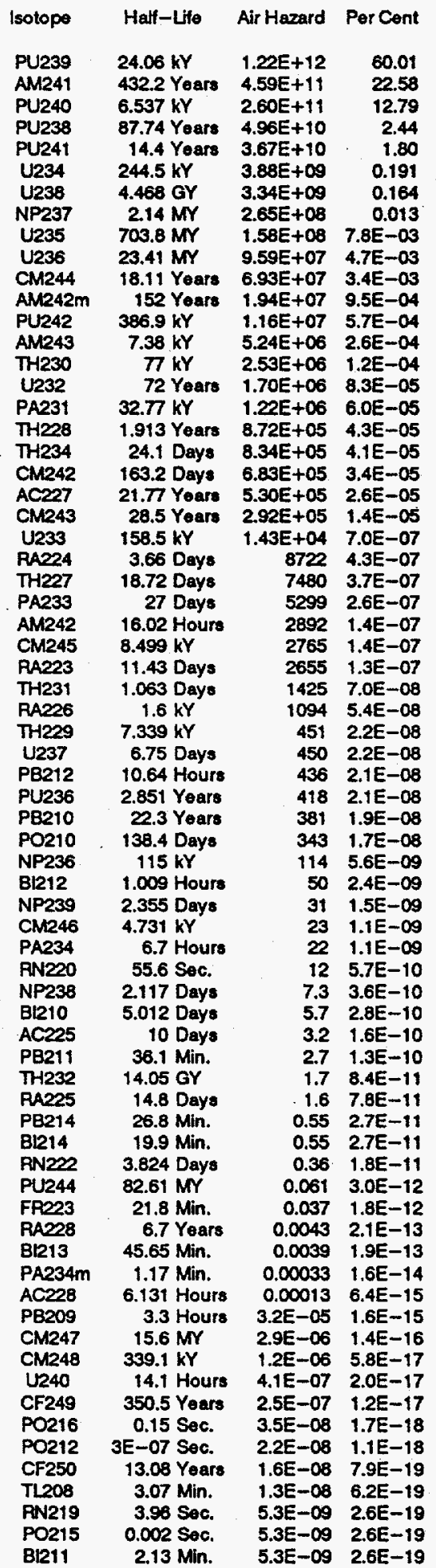

Table 6-F. Decay Time $=100$ Years

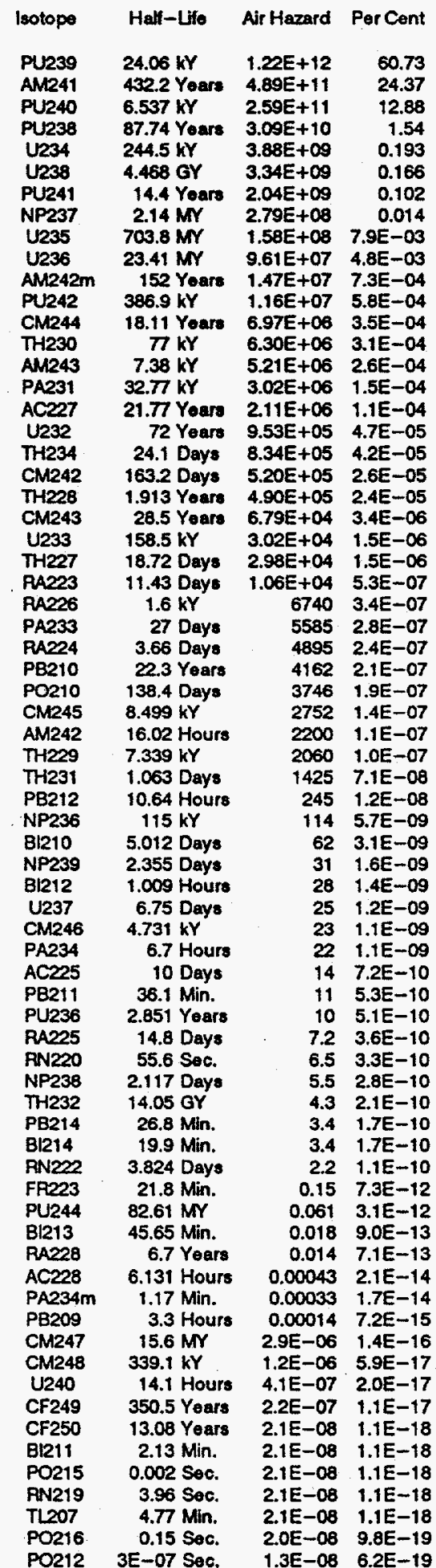


Table 2.6. (contd)

Table 6-G. Decay $7 \mathrm{me}=300$ Years

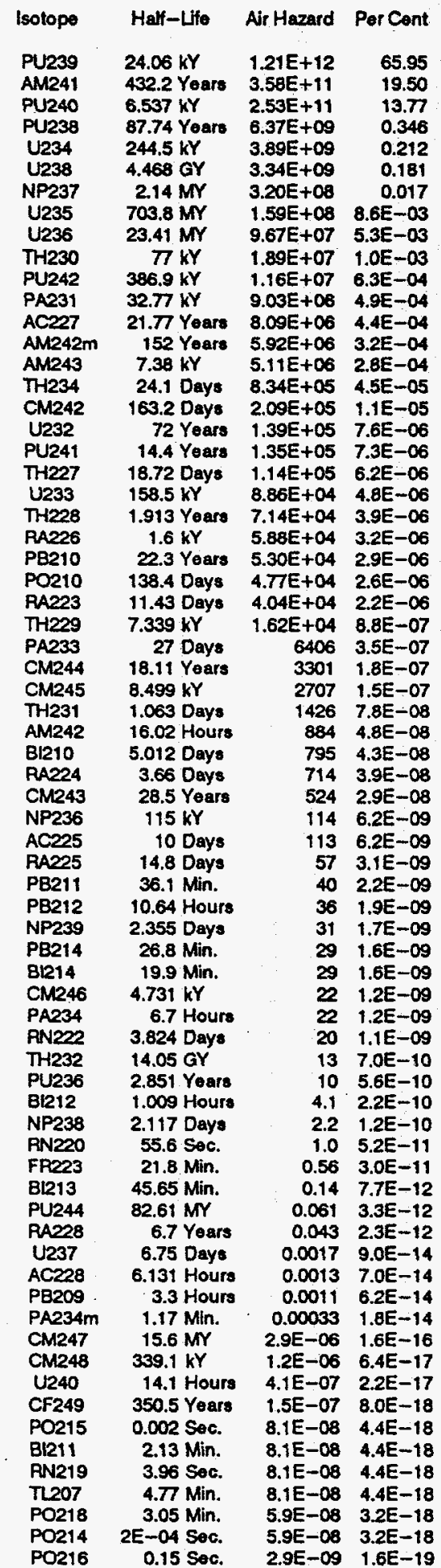

Table 6-H. Decay Time = 1000 Years

\begin{tabular}{|c|c|c|c|}
\hline & If - Lito & Air Hazard & Per Con \\
\hline & & 12 & \\
\hline & & & \\
\hline U23 & Yoars & $\begin{array}{l}-11 \\
-09\end{array}$ & $\begin{array}{r}7.53 \\
0.251\end{array}$ \\
\hline U23 & $Y$ & +09 & 0.215 \\
\hline P237 & $14 \mathrm{MY}$ & $94 \mathrm{E}+08$ & 0.025 \\
\hline & $8 \mathrm{MY}$ & $3 E+\infty 8$ & 0.010 \\
\hline & $M Y$ & & $4 E-03$ \\
\hline & 7. & $E+07$ & $4.1 E-0$ \\
\hline & oars & & -03 \\
\hline & & & $=-03$ \\
\hline & ares & & $=-03$ \\
\hline & & & -04 \\
\hline & & & \\
\hline & lays & & -05 \\
\hline & 2.3 .1 & & $E-05$ \\
\hline & & & \\
\hline & & & $E-05$ \\
\hline & ys & 4.2 & E-05 \\
\hline & & & $=-05$ \\
\hline & Perse & & $E-05$ \\
\hline & & & $1.2 \mathrm{E}-05$ \\
\hline & & & $E-06$ \\
\hline & $y=$ & 9860 & $E-07$ \\
\hline & & 8580 & $E-07$ \\
\hline & & 7869 & 5.1E-07 \\
\hline 45 & 8.49 & 2557 & 1.7E-07 \\
\hline & & 1429 & -08 \\
\hline & & 1311 & $E-08$ \\
\hline & & 656 & $E-08$ \\
\hline & & 96 & $E-0 B$ \\
\hline & & 96 & $E-08$ \\
\hline & & 17 & $E-08$ \\
\hline & & 197 & $E-C$ \\
\hline & & 149 & E-os \\
\hline & & 121 & $E-0 s$ \\
\hline & & 113 & $=-0 s$ \\
\hline & & 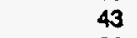 & -09 \\
\hline 41 & & 38 & $E-\infty$ \\
\hline & & 36 & $E-09$ \\
\hline & & 29 & $1.9 E-09$ \\
\hline & & 22 & $E-\infty$ \\
\hline & & 20 & $E-09$ \\
\hline & & 10 & $E-10$ \\
\hline & & 2.1 & $E-10$ \\
\hline & & 1.6 & $=-10$ \\
\hline & & 1.2 & \\
\hline & & 0.14 & \\
\hline & 170 & $x$ & -12 \\
\hline & & & \\
\hline & & & -12 \\
\hline & & & -13 \\
\hline & & & -13 \\
\hline & & & \\
\hline & & & \\
\hline & & & \\
\hline & & & \\
\hline & & & \\
\hline Ci & 335 & & \\
\hline & & & \\
\hline & & & -17 \\
\hline & & & \\
\hline & & & $5-11$ \\
\hline 15 & $2 \mathrm{se}$ & & $1.9 E-17$ \\
\hline & & & $1.9 \mathrm{E}-17$ \\
\hline & & & $1.9 \mathrm{E}-17$ \\
\hline & & & $1.9 E-17$ \\
\hline & & -08 & 2.3E-18 \\
\hline & (1) & & $4.9 E-19$ \\
\hline & & .09 & \\
\hline
\end{tabular}


Table 2.6. (contd)

Table 6-I. Decay Time $=3000$ Yeare

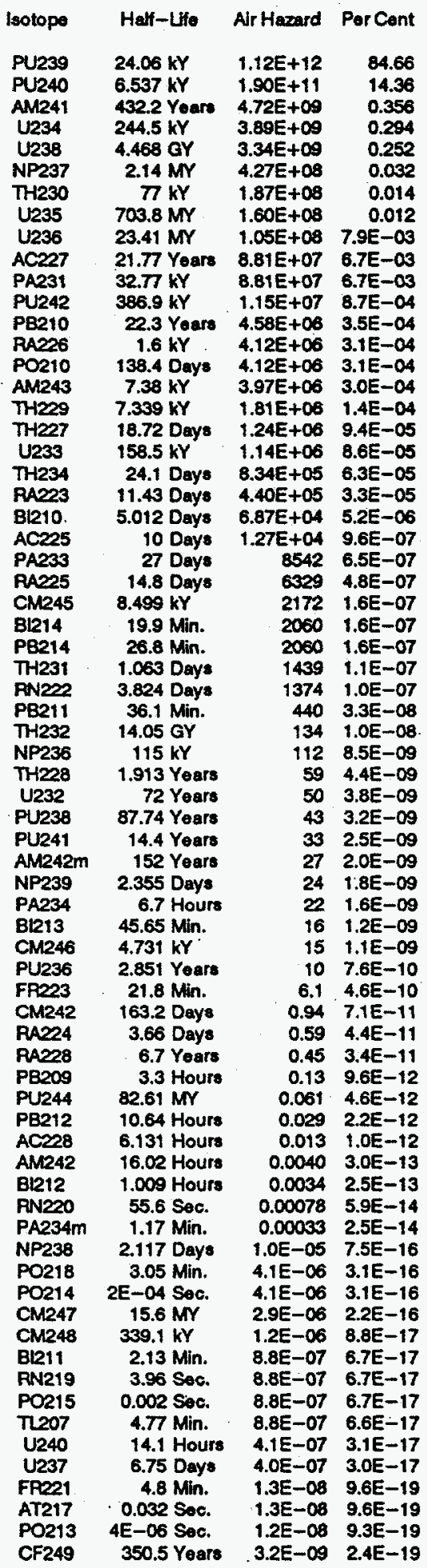


Table 2.7. Relative Ingestion Hazard of Fission Products in Hanford Reactor Fuel at Various Decay Times

Table 7-A. Decay Time $=1$ Yoar

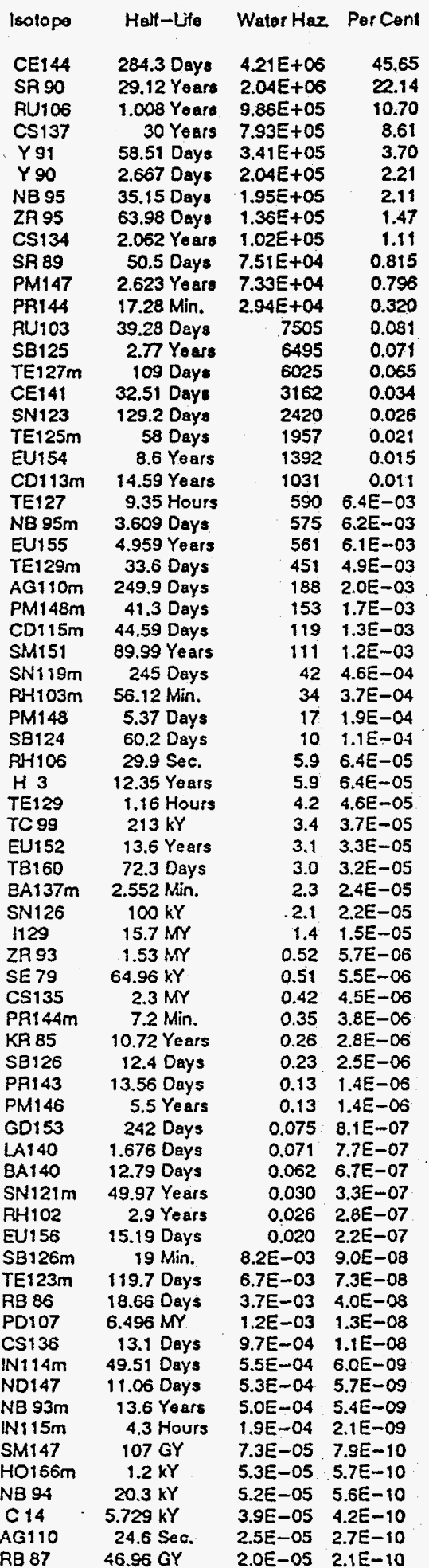

Tablo 7-B. Docay Time $=3$ Yoars.

\begin{tabular}{|c|c|c|c|}
\hline Ltope & Half-Lfe & Water Haz & Cen \\
\hline$S R$ & 29.12 Yoars & $1.94 E+\infty 6$ & 49.07 \\
\hline $\operatorname{cs} 137$ & & & 19.11 \\
\hline CE144 & 284.3 Daya & $7.08 E+05$ & 17.88 \\
\hline RU10s & 28 Years & +05 & 6.29 \\
\hline$x \sim$ & & & 4.91 \\
\hline CS134 & oars & & 1.32 \\
\hline PM147 & 2.623 Years & +04 & 1.09 \\
\hline PRi44 & $17.28 \mathrm{Min}$. & & 0.125 \\
\hline SB125 & 2.77 Years & 3937 & 0.099 \\
\hline TE125m & 3 Days & 1201 & 0.030 \\
\hline & & & 0.030 \\
\hline $3 m$ & 14. & 938 & 0.024 \\
\hline EU155 & Bars & 425 & 0.011 \\
\hline SM151 & & 109 & $2.8 E-03$ \\
\hline 11005 & & 73 & \\
\hline 10 & & 59 & $1.5 E-03$ \\
\hline TE127m & & 58 & $1.5 E-03$ \\
\hline $700 \mathrm{~s}$ & 63. & 50 & $1.3 E-03$ \\
\hline 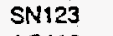 & 12 & 48 & $1.2 E-03$ \\
\hline$A G$ & & 25 & $6.3 E-04$ \\
\hline & & 5.7 & $1.4 E-04$ \\
\hline $9 m$ & & 5.4 & $1.4 E-04$ \\
\hline$H$ & 12. & 5.2 & $1.3 E-04$ \\
\hline & & 3.4 & 8.7E-05. \\
\hline & & 3.3 & 8 \\
\hline $\mathrm{E}$ & ears & 2.8 & $7.0 E-05$ \\
\hline BA137m & 2.55 & 2.1 & 5.4E-05 \\
\hline SN126 & 10 & 2.1 & $5.2 E-05$ \\
\hline RH106 & sec. & 1.5 & $3.8 E-05$ \\
\hline 1129 & 15 & 1.4 & $3.4 E-05$ \\
\hline ZR 93 & 1.5 & 0.52 & -05 \\
\hline 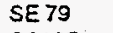 & & 0.51 & $1.3 E-05$ \\
\hline CS135 & $\gamma$ & 0.42 & $1.1 E-05$ \\
\hline S & & 0.23 & $5.6 E-06$ \\
\hline K & & 0.23 & $5.7 E-06$ \\
\hline m & & 0.21 & $5.3 E-06$ \\
\hline 1146. & ars & 0.098 & $2.5 E-06$ \\
\hline PR144m & 7.2 & 0.6 & $1.5 E-06$ \\
\hline SN1 & ars & & $7.4 \mathrm{E}-07$ \\
\hline RU1 & 39. & 0.019 & $4.8 E-07$ \\
\hline $\mathrm{RHI}$ & & & $E-07$ \\
\hline GL & 24 & 3 & $2.3 E-07$ \\
\hline$S B$ & & 3 & $2.3 E-07$ \\
\hline TB & & 3 & -08 \\
\hline & & 3 & $E-08$ \\
\hline $\mathrm{CO1}$ & 44.5 & -03 & $3.5 E-08$ \\
\hline VB & 13.6 & -03 & $=-08$ \\
\hline O1 & 6.4 & -03 & $3.0 E-08$ \\
\hline$m$ & ays & -04 & $1.8 E-08$ \\
\hline SE & 32.5 & -04 & -08 \\
\hline & & -04 & $E-09$ \\
\hline$=$ & 33 & -04 & $3.2 E-09$ \\
\hline TE1? & 119 & -05 . & $2.5 E-09$ \\
\hline & 5 & .05 & $2.2 E-09$ \\
\hline & 5.37 Days & $=-05$ & $2.1 E-09$ \\
\hline . & & -05 & 1.3E-09 \\
\hline NB 94 & $20.3 \mathrm{kY}$ & $5.2 E-05$ & $1.3 E-09$ \\
\hline & 5.72 & $=-05$ & $9.8 E-10$ \\
\hline RB 87 & $46.96 \mathrm{GY}$ & $2.0 E-05$ & $5.0 E-10$ \\
\hline AG108 & 127 Years & $1.1 E-05$ & $2.8 E-10$ \\
\hline G1 & & $3.3 E-\infty 6$ & 8.3E-11 \\
\hline & & $E-\infty 6$ & $5.7 E-11$ \\
\hline IEI2 & $1.16 r$ & $1.2 \mathrm{E}-06$ & $3.0 E-11$ \\
\hline & & & \\
\hline Uis & & $=-07$ & $1.1 E-11$ \\
\hline 98 & 19 & $E-07$ & $4.9 E-12$ \\
\hline$M 1$ & & $E-07$ & $2.5 E-12$ \\
\hline & & & $7.5 E-13$ \\
\hline & 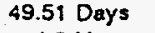 & $2.0 E-08$ & $5.0 E-13$ \\
\hline 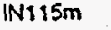 & 4.3 Hours & $2.2 E-\infty 9$ & -14 \\
\hline
\end{tabular}


Table 2.7. (contd)

Table 7-C. Decay Time $=10$ Years

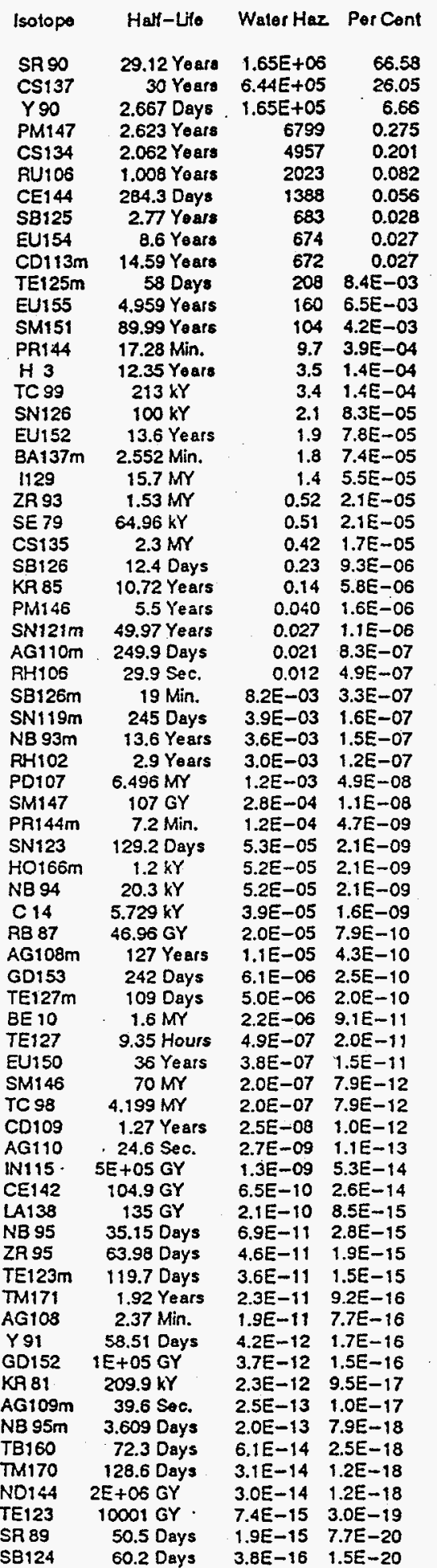

Table 7-D. Decey Time $=30$ Yoars

\begin{tabular}{|c|c|c|c|}
\hline otope & Hall-Lifo & Water Haz & Per Cor \\
\hline $\begin{array}{l}\text { CSt37 } \\
\text { Y90 }\end{array}$ & $\begin{array}{r}29.12 \text { Years } \\
30 \text { Yeare } \\
2.667 \text { Days }\end{array}$ & $\begin{array}{l}1.02 E+06 \\
4.06 E+05 \\
1.02 E+05\end{array}$ & $\begin{array}{r}66.77 \\
26.51 \\
6.68\end{array}$ \\
\hline CD113m & 14.59 Years & 260 & $\begin{array}{r}6.68 \\
0.017\end{array}$ \\
\hline & & 135 & $8.8 E-03$ \\
\hline & 89. & 89 & $5.8 E-03$ \\
\hline 4147 & ars & 34 & 2.3E-03 \\
\hline & & 9.7 & $6.4 E-04$ \\
\hline & & 6.0 & $3.9 E-04$ \\
\hline & & 4.6 & $3.0 E-04$ \\
\hline & & 3.4 & $2.2 E-04$ \\
\hline SNI & & 2.1 & $1.3 E-04$ \\
\hline TE125m & 58 Days. & 1.4 & 9.1E-05 \\
\hline 1129 & 15.7 & 1.4 & $8.9 E-05$ \\
\hline BA137m & & 1.2 & $7.5 E-05$ \\
\hline & & 1.2 & $E-05$ \\
\hline & & 0.70 & $4.6 E-05$ \\
\hline & & 0.52 & $3.4 E-05$ \\
\hline . & 64.9 & 0.51 & $3.3 E-05$ \\
\hline$S 135$ & & 0.42 & 2.7E-05 \\
\hline & & 0.23 & $1.5 E-05$ \\
\hline & ats & 0.039 & $2.6 E-06$ \\
\hline im & ears & 0.020 & 1.3E-06 \\
\hline & & $2 E-03$ & $5.4 E-07$ \\
\hline & & -03 & $4.6 E-07$ \\
\hline & & -03 & $2.1 E-07$ \\
\hline & 1.00 & -03 & $1.4 E-07$ \\
\hline & f & -03 & $E-08$ \\
\hline & & $=-04$ & $E-08$ \\
\hline $6 m$ & & -05 & E-09 \\
\hline & & -05 & $E-09$ \\
\hline & 5.7 & -05 & E-09 \\
\hline & 284 & -05 & $E-09$ \\
\hline & & -05 & $E-09$ \\
\hline & 46. & -05 & -09 \\
\hline n & ars & -06 & -10 \\
\hline 8 & & -05 & -10 \\
\hline & & -07 & -11 \\
\hline E & irs & -07 & -11 \\
\hline 9 & r & -07 & -11 \\
\hline 4 & n. & -07 & -11 \\
\hline & & -08 & -13 \\
\hline N11 & . & $=-09$ & -14 \\
\hline & & -10 & -14 \\
\hline 3 & & $2.1 E-10$ & -14 \\
\hline 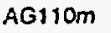 & 249.9 Days & $E-11$ & $2.1 E-15$ \\
\hline G108 & 2.37 Min. & $.7 E-11$ & -15 \\
\hline & $1 E+05 \mathrm{GY}$ & $4.6 E-12$ & $3.0 E-16$ \\
\hline SNI19m & 245 Days & $1.1 E-12$ & $2.7 E-16$ \\
\hline KR 81 & 209.9 & $3 E-12$ & $1.5 E-16$ \\
\hline & 7.2 & -12 & $1.4 E-16$ \\
\hline & ears & -13 & $3.0 E-17$ \\
\hline & $2 E+0$ & -14 & $1.9 E-18$ \\
\hline 171 & & -14 & $1.1 E-18$ \\
\hline & & & $4.9 E-19$ \\
\hline & & & $E-19$ \\
\hline 1149 & $1 E \div 07$ & -16 & $1.2 E-20$ \\
\hline & & -16 & $9.5 E-21$ \\
\hline & & & -22 \\
\hline & & 18 & $2.8 E-22$ \\
\hline & & & \\
\hline & & -26 & $2.2 E-30$ \\
\hline & & -27 & 2.2E-31 \\
\hline & & -29 & \\
\hline & 128.6 & $4 E-31$ & $1.6 \mathrm{E}-35$ \\
\hline & & 0 & \\
\hline & 12 & 0 & \\
\hline & & 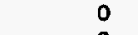 & \\
\hline & & 0 & \\
\hline & & 0 & \\
\hline
\end{tabular}


Table 2.7. (contd)

Table 7-E. Decay Time $=40$ Years

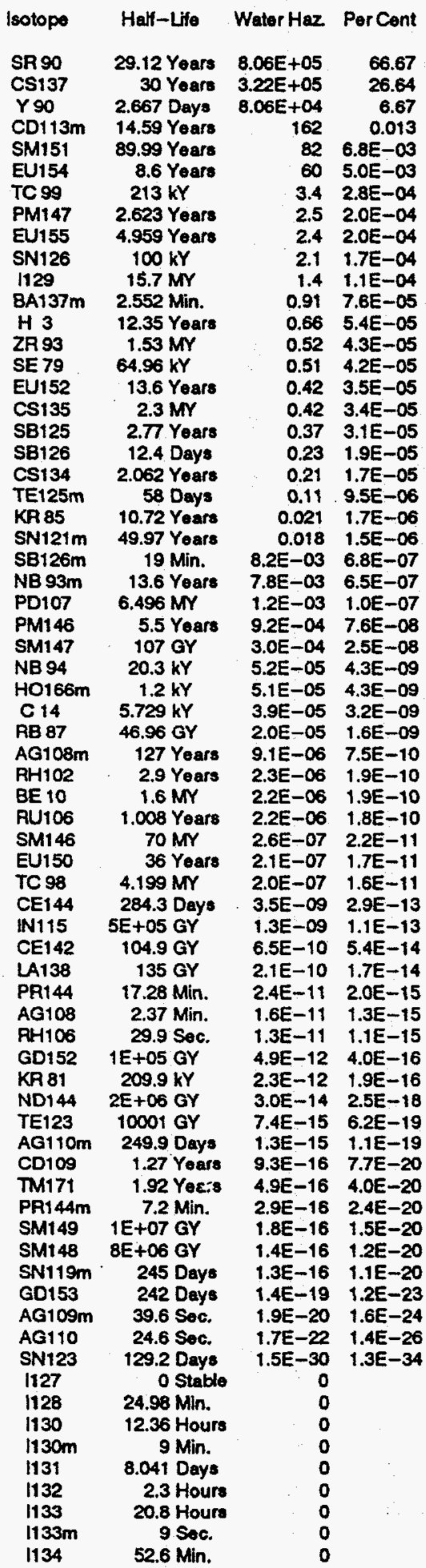

Table 7-F. Docay Time $=100$ Years

\begin{tabular}{|c|c|c|c|}
\hline otope & Half-Lito & Water Haz & Per Cent \\
\hline $\begin{array}{l}\text { SR } 90 \\
\text { CS137 }\end{array}$ & $\begin{array}{r}29.12 \text { Years } \\
30 \text { Years }\end{array}$ & $\begin{array}{r}193,200 \\
80,500\end{array}$ & $\begin{array}{l}65.92 \\
27.46\end{array}$ \\
\hline$Y 90$ & 2.667 Day & 19,330 & 6.60 \\
\hline SM151 & 89.99 Years & 52 & 0.018 \\
\hline CD113m & 14.59 Years & 9.3 & $3.2 E-03$ \\
\hline & 213 & 3.4 & $1.2 E-03$ \\
\hline SN126 & $100 \mathrm{kY}$ & 2.1 & 7.0E-04 \\
\hline 1129 & $15.7 \mathrm{MY}$ & 1.4 & 4.6E-04 \\
\hline & $1.53 \mathrm{MY}$ & 0.52 & $1.8 E-04$ \\
\hline SE 79 & $64.96 \mathrm{kY}$ & 0.51 & $1.7 E-04$ \\
\hline EU154 & 8.6 Yeare & 0.48 & $1.6 E-04$ \\
\hline CS135 & $2.3 \mathrm{MY}$ & 0.42 & $1.4 E-04$ \\
\hline SB126 & 12.4 Days & 0.23 & 7.9E-05 \\
\hline BA137m & 2.552 Min. & 0.23 & 7.8E-05 \\
\hline & ears & 0.023 & 7.7E-06 \\
\hline 52 & 'eara & 0.020 & $6.7 E-06$ \\
\hline NB 93m & 13.6 Years & $8.9 E-03$ & $3.0 E-06$ \\
\hline $126 \mathrm{~m}$ & 19 Min. & $8.2 E-03$ & $2.8 E-06$ \\
\hline $121 \mathrm{~m}$ & 49.97 Years & 7.7E-03 & $2.6 E-06$ \\
\hline PD107 & 6.49 & $1.2 E-03$ & 4.1E-07 \\
\hline 155 & ears & 5.5E-04 & $1.9 E-07$ \\
\hline 85 & Yoars & 4.3E-04 & $1.5 E-07$ \\
\hline 47 & 107 & $3.0 \mathrm{E}-04$ & $1.0 E-07$ \\
\hline 94 & 20.3 & $5.2 E-05$ & $1.8 E-08$ \\
\hline $166 m$ & 1. & 5.0E-05 & $\begin{array}{l}1.7 E-08 \\
1.3 E-08\end{array}$ \\
\hline 4 & 5.7 & $3.8 \mathrm{E}-05$ & $\begin{array}{l}1.3 E-08 \\
6.7 E-09\end{array}$ \\
\hline $\begin{array}{l}\text { RB } 87 \\
\text { AG108m }\end{array}$ & $\begin{array}{l}46.96 \mathrm{GY} \\
127 \text { Years }\end{array}$ & $\begin{array}{l}2.0 E-05 \\
6.6 E-06\end{array}$ & $\begin{array}{l}6.7 E-09 \\
2.2 E-09\end{array}$ \\
\hline BE 10 & $1.6 \mathrm{MY}$ & $2.2 E-06$ & $7.7 E-10$ \\
\hline 1146 & 5.5 Years & $4.8 E-07$ & $1.6 E-10$ \\
\hline 147 & 2.623 Years & $3.2 E-07$ & $1.1 E-10$ \\
\hline 146 & $70 \mathrm{MY}$ & $2.6 E-07$ & $9.0 E-11$ \\
\hline 98 & $4.199 \mathrm{MY}$ & $2.0 E-07$ & $6.7 E-11$ \\
\hline 125 & 2.77 Years & $1.1 E-07$ & $3.9 E-11$ \\
\hline EU150 & 36 Years & $6.6 E-08$ & $2.3 E-11$ \\
\hline TE125m & 58 Days & $3.4 E-08$ & $1.2 E-11$ \\
\hline W115 & 5E+05 GY & $1.3 E-09$ & $4.4 E-13$ \\
\hline 142 & 104 & $6.5 E-10$ & $2.2 E-13$ \\
\hline 134 & 2.062 Years & $3.6 E-10$ & $1.2 E-13$ \\
\hline LA138 & $135 \mathrm{Gr}$ & 2.1E-10 & $7.2 E-14$ \\
\hline 108 & 2.37 Min. & $1.2 E-11$ & $4.0 E-15$ \\
\hline 152 & $1 E+05 \mathrm{GY}$ & $5.2 E-12$ & $1.8 E-15$ \\
\hline 81 & $209.9 \mathrm{kY}$ & $2.3 E-12$ & $8.0 E-16$ \\
\hline 1102 & 2.9 Years & $1.4 E-12$ & $4.6 E-16$ \\
\hline 144 & $2 E+06 \mathrm{GY}$ & $3.0 E-14$ & $1.0 E-17$ \\
\hline$=123$ & $10001 \mathrm{GY}$ & $7.4 E-15$ & $2.5 E-18$ \\
\hline 149 & $1 E+07$ GY & $1.8 E-16$ & $6.2 E-20$ \\
\hline 1148 & $8 E+06 \mathrm{GY}$ & $1.4 E-16$ & $4.9 E-20$ \\
\hline 106 & 1.008 Years & -24 & $9.1 E-28$ \\
\hline TM171 & 1.92 Yoars & -25 & $6.0 E-29$ \\
\hline 1106 & 29.5 & -29 & $\begin{array}{l}5.5 E-33 \\
4.0 E-33\end{array}$ \\
\hline & & & \\
\hline $\begin{array}{l}\text { CS133 } \\
\text { BA133 }\end{array}$ & te & & \\
\hline $\begin{array}{l}133 \\
133 m\end{array}$ & $\begin{array}{r}10.7 \\
2.1\end{array}$ & 0 & \\
\hline $\begin{array}{l}133 m \\
34\end{array}$ & $\begin{array}{r}2.1 \\
0.07\end{array}$ & 0 & \\
\hline & $\begin{array}{l}0.07 \\
5.24\end{array}$ & 0 & \\
\hline $\begin{array}{l}133 \\
33 m\end{array}$ & $\begin{array}{l}\text { Days } \\
\text { Sec. }\end{array}$ & 0 & \\
\hline $\begin{array}{l}33 m \\
134\end{array}$ & & 0 & \\
\hline $\begin{array}{l}134 \\
131 n\end{array}$ & $\begin{array}{l}11 \text { Sec. } \\
11.9 \text { Days }\end{array}$ & 0 & \\
\hline & $\begin{array}{l}11.9 \text { Days } \\
8.041 \text { Days }\end{array}$ & 0 & \\
\hline $\begin{array}{l}1131 \\
\text { IN132 }\end{array}$ & $\begin{array}{l}\text { Says } \\
\text { Sec. }\end{array}$ & 0 & \\
\hline $\begin{array}{l}\text { IN132 } \\
\text { CD132 }\end{array}$ & 0.14 & 0 & \\
\hline $\begin{array}{l}\text { CD132 } \\
\text { SB132 }\end{array}$ & 2.8 & $\mathbf{U}$ & \\
\hline XE131 & able & 0 & \\
\hline TE132 & 3.258 Daya & 0 & \\
\hline TE131m & 1.25 Days & 0 & \\
\hline TE131 & 25 & 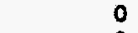 & \\
\hline & & 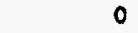 & \\
\hline 1133 & 20.8 & 0 & \\
\hline
\end{tabular}


Table 2.7. (contd)

Table 7-G. Decay Time $=300$ Yeare

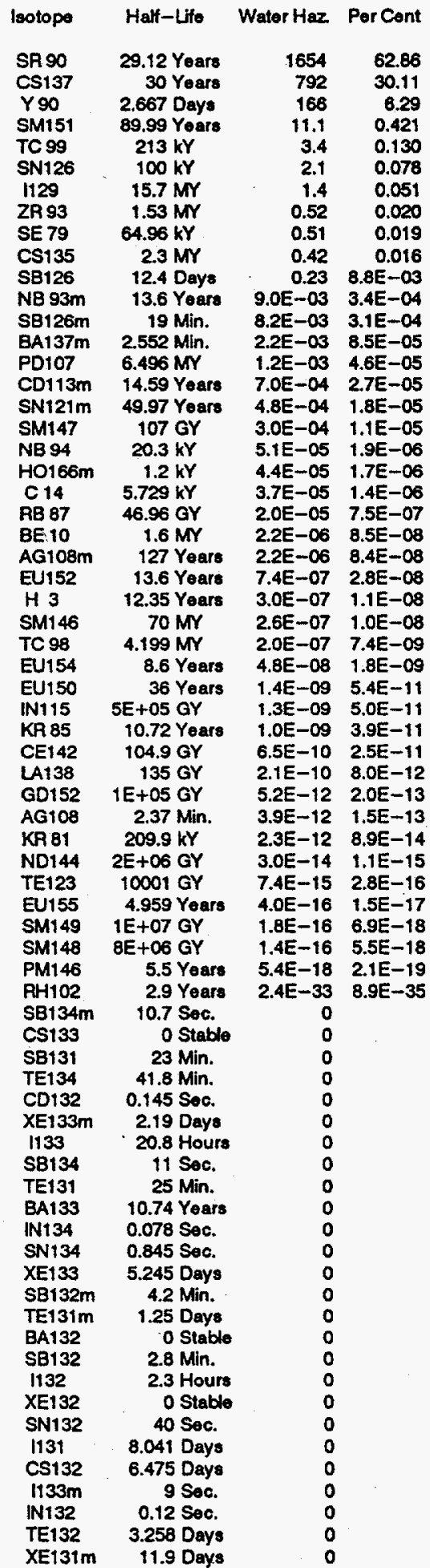

Table 7-H. Decay Time $=1000$ Years

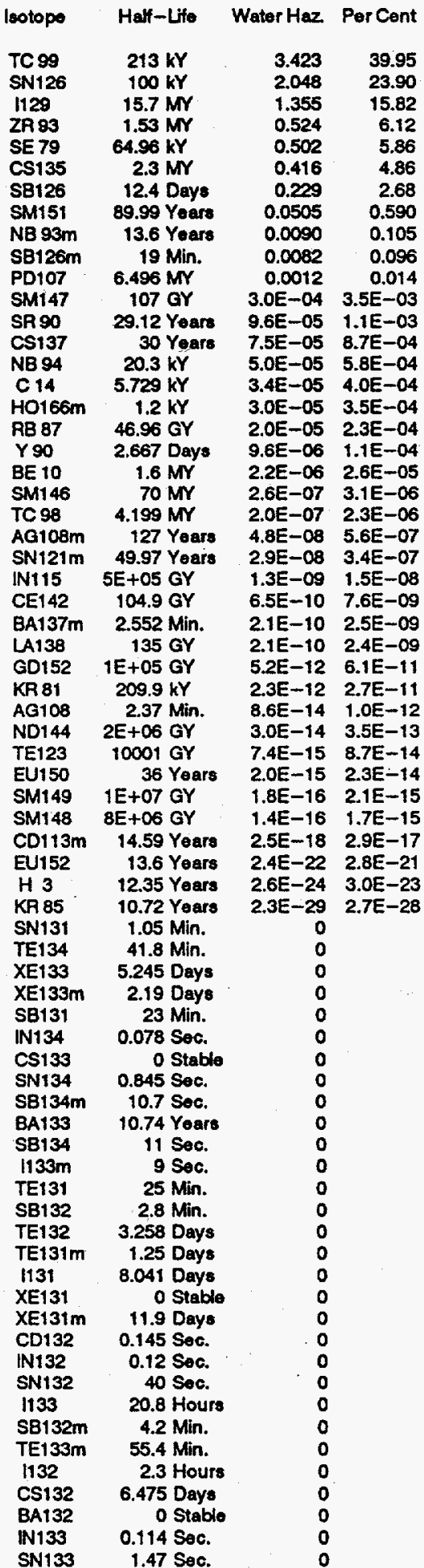


Table 2.7. (contd)

Table 7-1. Decay Time $=3000$ Yoars

\begin{tabular}{|c|c|c|c|}
\hline Isotope & Hatt-Lifo & Water Haz & Por Cont \\
\hline TC 9 & $213 \mathrm{kY}$ & 11 & 40.2 \\
\hline SN126 & $100 \mathrm{k}$ & 2.020 & 23.90 \\
\hline 1129 & $15.7 \mathrm{MY}$ & 1.355 & 16.03 \\
\hline ZR 93 & $1.53 \mathrm{MY}$ & 0.524 & 6.20 \\
\hline SE 79 & $64.96 \mathrm{kY}$ & 0.492 & 5.82 \\
\hline CS135 & $2.3 \mathrm{MV}$ & 0.416 & 4.92 \\
\hline SB126 & 12.4 Days & 0.226 & 2.68 \\
\hline NB $93 \mathrm{~m}$ & 13.6 Years & 0.0090 & 0.106 \\
\hline SB126m & 19 Min. & 0.0081 & 0.096 \\
\hline PD107 & $6.496 \mathrm{MY}$ & 0.0012 & 0.014 \\
\hline SM147 & $107 \mathrm{GY}$ & $\begin{array}{l}3.0 E-04 \\
47 E-05\end{array}$ & $\begin{array}{l}3.5 E-03 \\
5.5 E-04\end{array}$ \\
\hline $\begin{array}{c}\text { NB } 94 \\
\text { C } 14\end{array}$ & $5.729 \mathrm{kY}$ & $2.7 E-05$ & $\begin{array}{l}3.5 E-04 \\
3.2 E-04\end{array}$ \\
\hline RB 87 & 46.96 OY & 2.0E-05 & $2.3 \mathrm{E}-04$ \\
\hline $\begin{array}{l}\text { HO166m } \\
\text { BE } 10\end{array}$ & $1.2 \mathrm{kY}$ & 9.3E-06 & $\begin{array}{l}1.1 E-04 \\
2.7 E-05\end{array}$ \\
\hline SM146 & $\begin{array}{l}1.6 \mathrm{MH} \\
70 \mathrm{MY}\end{array}$ & $\begin{array}{l}2.2 E-08 \\
2.6 E-07\end{array}$ & $\begin{array}{l}2.7 E-05 \\
3.1 E-06\end{array}$ \\
\hline TC 98 & $4.199 \mathrm{MY}$ & $2.0 E-07$ & $2.3 E-06$ \\
\hline SM151 & 89.99 Years & $1.0 \mathrm{E}-08$ & $1.2 E-07$ \\
\hline IN115 & $5 E+05 G Y$ & $1.3 E-09$ & 1.5E-08 \\
\hline CE142 & $104.9 \mathrm{GY}$ & $6.5 E-10$ & 7.7E-09 \\
\hline LA138 & $135 \mathrm{GY}$ & $2.1 E-10$ & 2.5E-09 \\
\hline GD152 & $1 E+0$ & $5.2 E-12$ & $6.1 E-11$ \\
\hline KR 81 & $209.9 \mathrm{kY}$ & $2.3 E-12$ & 2.7E-11 \\
\hline AG108m & 127 Yoaro & $8.8 E-13$ & $1.0 \mathrm{E}-11$ \\
\hline D144 & $2 E+c$ & $3.0 E-14$ & $3.5 E-13$ \\
\hline TE123 & $10001 \mathrm{GY}$ & $7.4 E-15$ & $8.8 E-14$ \\
\hline $\sin 149$ & $1 E+07 \mathrm{GY}$ & $1.8 \mathrm{E}-16$ & $2.2 E-15$ \\
\hline SM148 & $8 E+06$ GY & $1.4 E-16$ & $1.7 E-15$ \\
\hline AG108 & 2.37 Min. & $1.6 E-18$ & $1.8 E-17$ \\
\hline $\begin{array}{l}\text { SN121m } \\
\text { CS137 }\end{array}$ & $\begin{array}{l}49.97 \text { Yeare } \\
30 \text { Years }\end{array}$ & $\begin{array}{l}2.6 E-20 \\
6.4 E-25\end{array}$ & $\begin{array}{r}3.1 E-19 \\
7.6 E-24\end{array}$ \\
\hline $\begin{array}{l}\text { CS137 } \\
\text { SR 90 }\end{array}$ & $\begin{array}{r}30 \text { Years } \\
29.12 \text { Years }\end{array}$ & $\begin{array}{l}6.4 E-25 \\
2.0 E-25\end{array}$ & $\begin{array}{l}7.6 E-24 \\
2.4 E-24\end{array}$ \\
\hline Y 90 & 2.667 Days & $2.0 E-26$ & $2.4 E-25$ \\
\hline BA137m & 2.552 Min. & $1.8 E-30$ & $2.1 \varepsilon-29$ \\
\hline EU150 & & $3.7 E-32$ & 4.4E-31 \\
\hline XE133m & 2.19 Days & & \\
\hline IN134 & 0.078 Sec. & 0 & \\
\hline N131 & 0.3 Sec. & 0 & \\
\hline CS 133 & 0 Stable & 0 & \\
\hline SN131 & 105 & 0 & \\
\hline SB134m & 10.7 Sec. & 0 & \\
\hline $\begin{array}{l}\text { BA133 } \\
\text { SB131 }\end{array}$ & $\begin{array}{c}10.74 \text { Yoars } \\
23 \text { Min. }\end{array}$ & $\begin{array}{l}0 \\
0\end{array}$ & \\
\hline $\begin{array}{l}\text { SB131 } \\
\text { SB134 }\end{array}$ & 11 Soc. & 0 & \\
\hline RA130 & O Stable & 0 & \\
\hline SN134 & 0.845 Sec. & 0 & \\
\hline TF123 &  & 0 & \\
\hline XE133 & 5.24 & 0 & \\
\hline 1131 & 8.041 Days & 0 & \\
\hline $1133 \mathrm{~m}$ & $9 \mathrm{se}$ & 0 & \\
\hline TE132 & 3.258 Days & 0 & \\
\hline XE132 & O Stable & 0 & \\
\hline XE131m & 11.9 Days & 0 & \\
\hline CD132 & $0.145 \mathrm{~S}$ & 0 & \\
\hline TE131m & 1.25 & 0 & \\
\hline IN132 & $0.12 \mathrm{~S}$ & 0 & \\
\hline SN13 & $40 \mathrm{~S}$ & 0 & \\
\hline SB132 & 2.8 Min. & 0 & \\
\hline SB132m & $4.2 \mathrm{M}$ & 0 & \\
\hline 113 & $20.8 \mathrm{H}$ & 0 & \\
\hline 1132 & 2.3 Hours & 0 & \\
\hline TE133m & $55.4 \mathrm{Mi}$ & 0 & \\
\hline TE131 & $25 \mathrm{M}$ & 0 & \\
\hline CS132 & 6.475 Days & 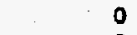 & \\
\hline XE131 & 0 Stable & 0 & \\
\hline IN133 & 0.114 Sec. & 0 & \\
\hline$\sqrt{133}$ & 1.47 & 0 & \\
\hline SB133 & 2.4 & 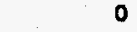 & \\
\hline TE133 & $12.45 \mathrm{~A}$ & 0 & \\
\hline
\end{tabular}


Table 2.8. Relative Ingestion Hazard of Activation Products in Hanford Reactor Fuel and Cladding at Various Decay Times

Tabio 8-A. Decay Timo = 1 Year

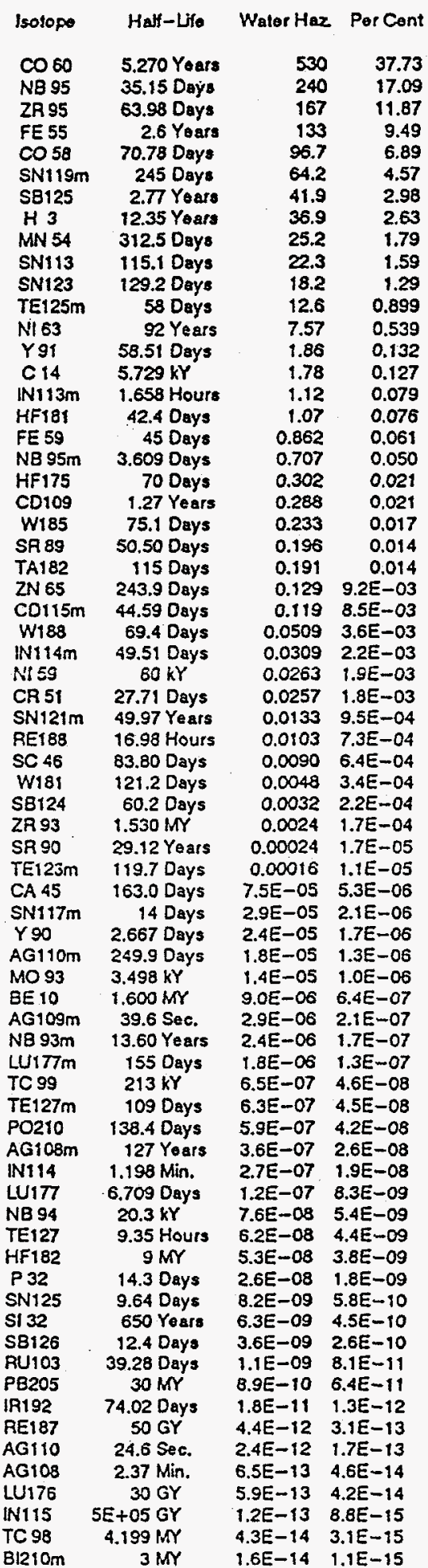

Table 8-B. Decay Time $=3$ Years

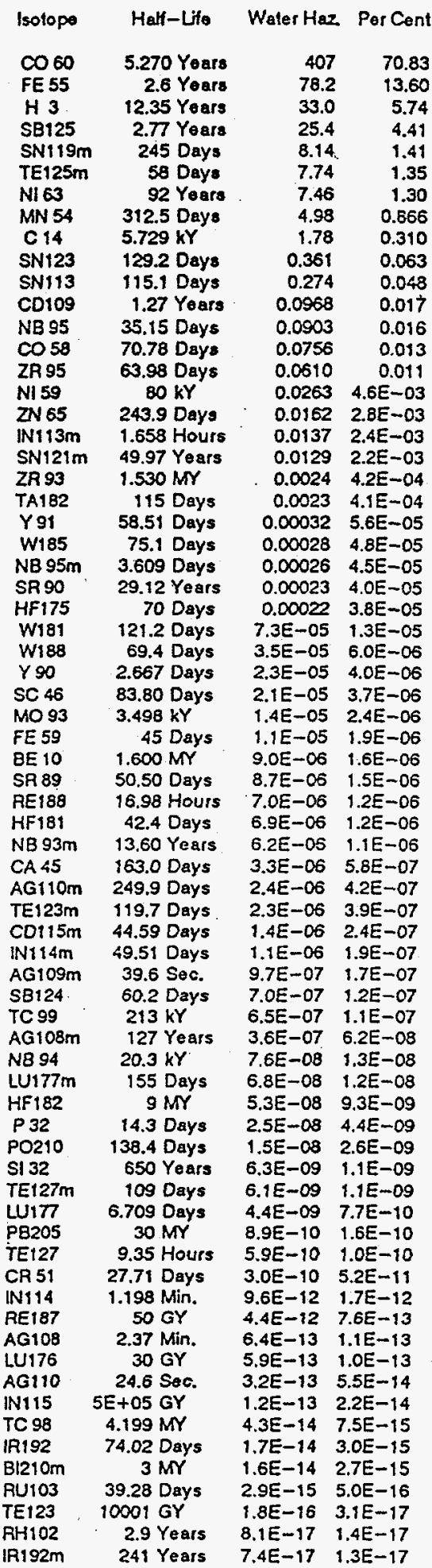


Table 2.8. (contd)

Tabio 8-C. Docay Timo $=10$ Yárs

\begin{tabular}{|c|c|c|c|}
\hline 1000 & $f f-$ Lfo & Haz & or $C_{a}$ \\
\hline & & & \\
\hline & 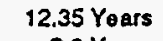 & 22.3 & \\
\hline & $y$ & 12.1 & \\
\hline & 2Y & 7.08 & \\
\hline 8125 & 2.77 & 4.40 & \\
\hline$C_{14}$ & 5.729 & & \\
\hline TE125m & 58 & 1.34 & \\
\hline NI 5 & & 0.0263 & \\
\hline MN 54 & 312.5 Day» & 0.0172 & $8.1 E-C$ \\
\hline SN121m & $49.97 Y$ & 0.0117 & $5.5 \mathrm{E}-\mathrm{C}$ \\
\hline SN119m & & 0.0059 & $295-0$ \\
\hline ZR 93 & $1.530 \mathrm{Mr}$ & 0.0024 & $1.2 E-0$ \\
\hline C0109 & & & \\
\hline CPOQ & & & -0 \\
\hline & & & \\
\hline NB 93m & 13.6 & & $7.9 E-\infty 6$ \\
\hline MO & 3.49 & -05 & $6.6 \mathrm{E}-\infty$ \\
\hline & & & $E-\infty$ \\
\hline BE & 1.60 & & -0 \\
\hline TCS & 213 & & $3.1 E-0$ \\
\hline SN1 & & & \\
\hline AG108m & 127 & -07 & $1.6 \mathrm{E}-0$ \\
\hline NBS & & & $3.6 \mathrm{E}-08$ \\
\hline SNI & & & -0 \\
\hline IF 1 & & & $2.5 \mathrm{E}-0$ \\
\hline TA1 & & & $=-08$ \\
\hline$P .3$ & 14.3 & & $1.2 E-08$ \\
\hline $9 \mathrm{~m}$ & & & \\
\hline & & & $3.0 E-0 S$ \\
\hline N11 & 1.65 & & $1.3 E-05$ \\
\hline & & -09 & $9.4 E-10$ \\
\hline 820 & 301 & -10 & $4.2 E-10$ \\
\hline$\omega_{1}+2$ & 163. & & $3.0 E-11$ \\
\hline & & & $1.5 E-11$ \\
\hline FEI 1 & & & $2.1 E-12$ \\
\hline & & -12 & -13 \\
\hline E12 & 119 & -13 & $4.0 E-13$ \\
\hline 17 & & & \\
\hline & & & \\
\hline $117-2-3$ & 30 & & \\
\hline & 5 & & \\
\hline & & -14 & -14 \\
\hline & & & \\
\hline & & & \\
\hline 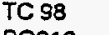 & & -14 & -14 \\
\hline & & & \\
\hline 21 & & -14 & -15 \\
\hline & & -14 & \\
\hline & & & -15 \\
\hline & & & \\
\hline & & & \\
\hline & & -16 & \\
\hline 1 & & & \\
\hline & & & \\
\hline & & & \\
\hline & & & -17 \\
\hline & & & \\
\hline & & & \\
\hline & & & \\
\hline & & & \\
\hline & & & \\
\hline & & & \\
\hline & & & \\
\hline & & & \\
\hline & $v^{2}+2$ & 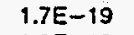 & \\
\hline & & & \\
\hline & $60,2 \mathrm{Da}$ & $1.15=-19$ & $5.4 E-20$ \\
\hline & & & \\
\hline & - & $3.1 E-20$ & anc \\
\hline & 50.50 Davs & $5.0 E-21$ & $24 E-21$ \\
\hline
\end{tabular}

Tabie 8-D. Decay Time $=30$ Years

\begin{tabular}{|c|c|c|c|}
\hline otope & Halt-Lfo & Wator Haz & PerCent \\
\hline & 5.270 Yoars & $\$ 1.69$ & 43.39 \\
\hline$=$ & 12.35 Yoars & 7.25 & 26.92 \\
\hline NI 63 & 92 Years & 6.09 & 22.60 \\
\hline$C_{14}$ & $5.729 \mathrm{kY}$ & 1.78 & 6.59 \\
\hline FE 55 & 2.6 Yoars & 0.0585 & 0.217 \\
\hline SB125 & 2.77 Yoars & 0.0295 & 0.110 \\
\hline NI 59 & $80 \mathrm{kY}$ & 0.0263 & 0.098 \\
\hline TE125m & 58 Days & 0.0090 & 0.033 \\
\hline SN121m & 49.97 Years & 0.0089 & 0.033 \\
\hline ZR 93 & $1.530 \mathrm{Mr}$ & 0.0024 & $9.0 E-03$ \\
\hline SR9O & 29.12 Years & 0.00012 & $4.5 E-04$ \\
\hline NB 93m & 13.60 Years & 3.3E-05 & 1. $2 E-04$ \\
\hline MO 93 & $3.498 \mathrm{kY}$ & $1.4 E-05$ & $5.2 E-05$ \\
\hline$Y 90$ & 2.667 Days & 1.2E-05 & $4.5 E-05$ \\
\hline$B E 10$ & $1.600 \mathrm{Mr}$ & $9.0 E-06$ & $3.4 E-05$ \\
\hline TC 99 & $213 \mathrm{kY}$ & $6.5 E-07$ & $2.4 E-06$ \\
\hline AG108m & 127 Yoars & $3.1 E-07$ & $1.1 E-06$ \\
\hline NB 94 & $20.3 \mathrm{kY}$ & $7.6 E-08$ & $2.8 E-07$ \\
\hline HF182 & $9 \mathrm{Mr}$ & $5.3 E-08$ & $2.0 E-07$ \\
\hline CD109 & 1.27 Yoars & $3.9 E-08$ & $1.4 \mathrm{E}-07$ \\
\hline TA182 & 115 Days & $2.7 E-08$ & $9.9 E-08$ \\
\hline P 32 & 34.3 Days & $2.5 E-08$ & $9.1 E-08$ \\
\hline Si 32 & 650 Years & $6.1 \mathrm{E}-09$ & $2.3 E-08$ \\
\hline$M N 54$ & 312.5 Days & $1.6 E-09$ & $5.9 E-09$ \\
\hline PB205 & $30 \mathrm{MV}$ & $8.9 E-10$ & $3.3 E-09$ \\
\hline SN119m & 245 Days & $6.2 E-12$ & $2.3 E-11$ \\
\hline RE187 & $50 \mathrm{GY}$ & $4.4 E-12$ & $1.6 E-11$ \\
\hline LU176 & $30 \mathrm{GY}$ & $5.9 E-13$ & $2.2 E-12$ \\
\hline$A G 108$ & $2.37 \mathrm{Min}$. & $5.5 E-13$ & $2.0 E-12$ \\
\hline AG109m & 39.6 Sec. & $3.9 E-13$ & $1.4 \mathrm{E}-12$ \\
\hline NN115 & $5 E+05 G Y$ & $1.2 E-13$ & $4.6 \mathrm{E}-13$ \\
\hline TC 98 & $4.199 \mathrm{MY}$ & $4.3 E-14$ & $1.6 E-13$ \\
\hline $\mathrm{B} 1210 \mathrm{~m}$ & $3 \mathrm{Mr}$ & $1.6 E-14$ & $5.8 \mathrm{E}-14$ \\
\hline ZN 65 & 243.9 Days & $1.1 E-14$ & $4.0 \mathrm{E}-14$ \\
\hline PO210 & 138.4 Days & $1.6 E-15$ & $5.8 \mathrm{E}-15$ \\
\hline IR192 & 74.02 Days & $2.1 E-16$ & $7.8 E-16$ \\
\hline TE123 & $10001 \mathrm{GY}$ & $1.8 E-16$ & $6.6 E-16$ \\
\hline $\mathrm{AH} 102$ & 2.9 Years & $8.1 E-17$ & $3.0 E-16$ \\
\hline IR192m & 241 Years & $7.0 \mathrm{E}-17$ & $2.6 E-16$ \\
\hline PD107 & $6.496 \mathrm{MY}$ & $7,1 E-18$ & $2.6 E-17$ \\
\hline AGitom & 249.9 Days & $3.1 E-18$ & $1.2 \mathrm{E}-17$ \\
\hline PT193 & 500 Years & $4.0 E-19$ & $1.5 E-18$ \\
\hline 1129 & $15.7 \mathrm{MY}$ & 2.3E-19 & $8.5 E-19$ \\
\hline$\checkmark 50$ & $4 E+07 \mathrm{GY}$ & $1.7 E-19$ & $6.3 E-19$ \\
\hline P8204 & $1 E+0 B G Y$ & 1.3E-19 & $4.8 E-19$ \\
\hline B1208 & $368 \mathrm{kY}$ & $4.7 E-20$ & $1.8 E-19$ \\
\hline TL206 & 4.19 Min. & $3.1 E-20$ & $1.2 E-19$ \\
\hline$K 42$ & 12.36 Hours & $6.7 E-22$ & $2.5 E-29$ \\
\hline SN123 & 129.2 Days & $3.8 E-24$ & $1.4 \mathrm{E}-23$ \\
\hline CA 45 & 163.0 Days & 2.0E-24 & $7.5 E-24$ \\
\hline$A G 1: 0$ & $24.6 \mathrm{Sec}$. & $4.2 \mathrm{E}-25$ & $1.6 \mathrm{E}-24$ \\
\hline AR 42 & 33 Years & $6.7 E-26$ & $2.5 E-25$ \\
\hline LU177mm & 155 Days & $4.8 E-27$ & $1.8 E-26$ \\
\hline SN113 & 115.1 Days & $4.4 E-27$ & 1. $6 \mathrm{E}-26$ \\
\hline$T M 178$ & 1.92 Yoars & $2.2 E-27$ & $8.0 E-27$ \\
\hline RU106 & 1.008 Years & $8.8 E-28$ & $3.3 E-27$ \\
\hline LUiti & 6.709 Days & $3.1 E-28$ & $1.2 \mathrm{E}-27$ \\
\hline IN113m & 1.658 Hours & $2.2 E-28$ & $8.2 E-28$ \\
\hline W181 & 121.2 Days & $2.3 E-29$ & 8.7E-29 \\
\hline$T E 123 m$ & 119.7 Days & $3.5 E-31$ & $1.3 E-30$ \\
\hline RH106 & 29.9 Sec. & $5.3 E-33$ & $2.0 E-32$ \\
\hline BA135 & o Stabio & 0 & \\
\hline $3 \mathrm{~A} 135 \mathrm{~m}$ & 1.196 Days & 0 & \\
\hline $3 \mathrm{~A} 131 \mathrm{~m}$ & $15 \mathrm{Min}$. & 0 & \\
\hline A138 & $135 \mathrm{GY}$ & 0 & \\
\hline BA132 & o Stable & 0 & \\
\hline A133 & 10.74 Years & 0 & \\
\hline $3 A 133 \pi$ & 1.62 Days & 0 & \\
\hline M146 & $70 \mathrm{Mr}$ & 0 & \\
\hline & C St & 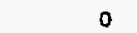 & \\
\hline
\end{tabular}


Table 2.8. (contd)

Table 8-E. Decay Time $=40$ Years

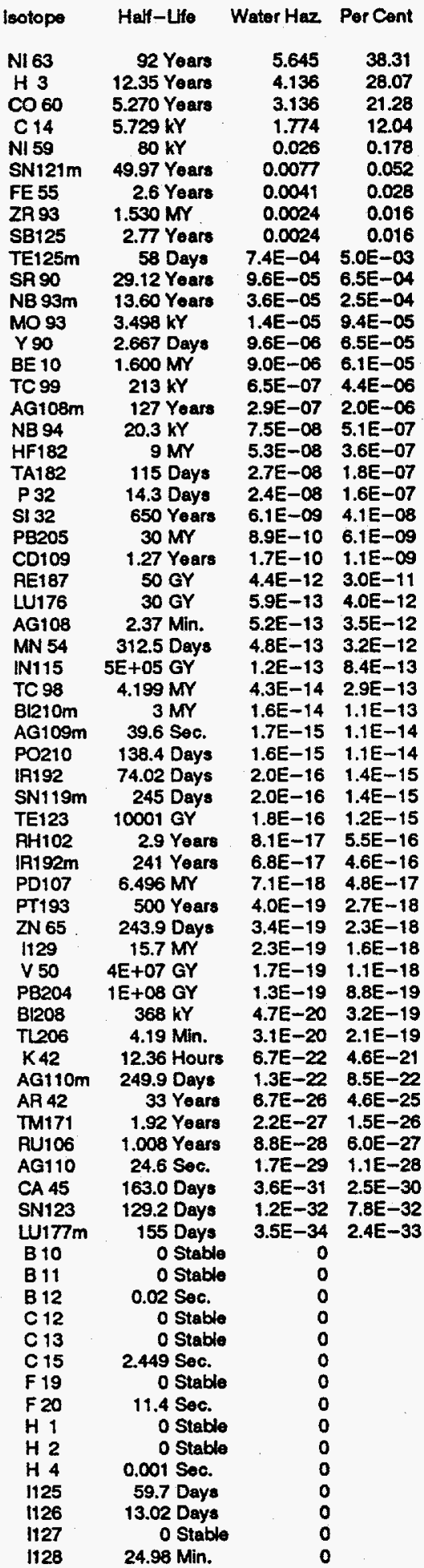

Table 8-F. Decay Time $=100$ Years

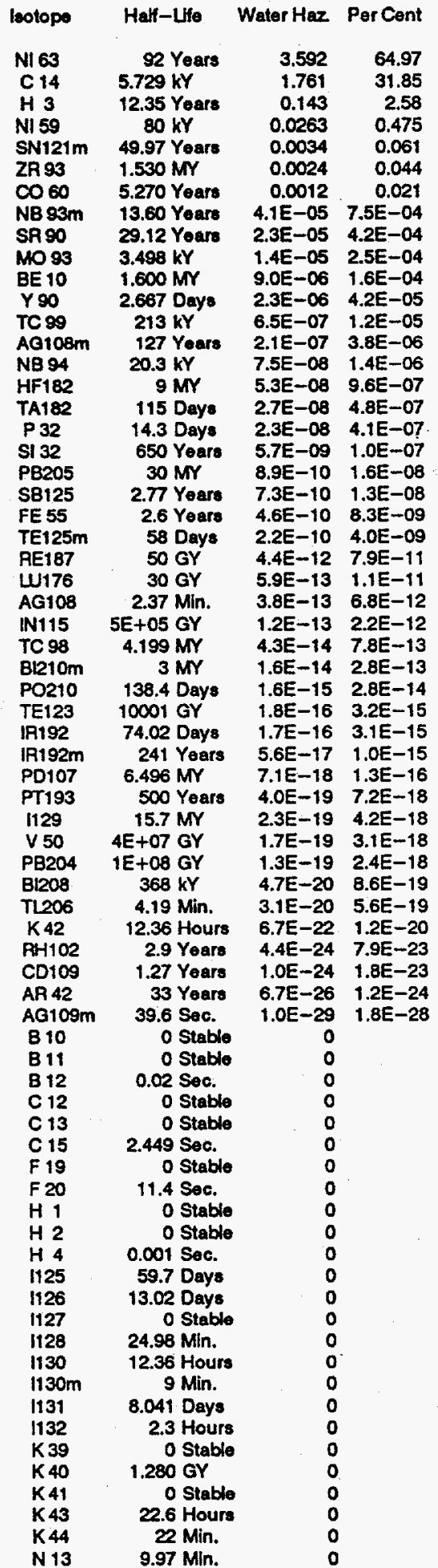


Table 2.8. (contd)

Table 8-G. Decay Time $=300$ Yeärs

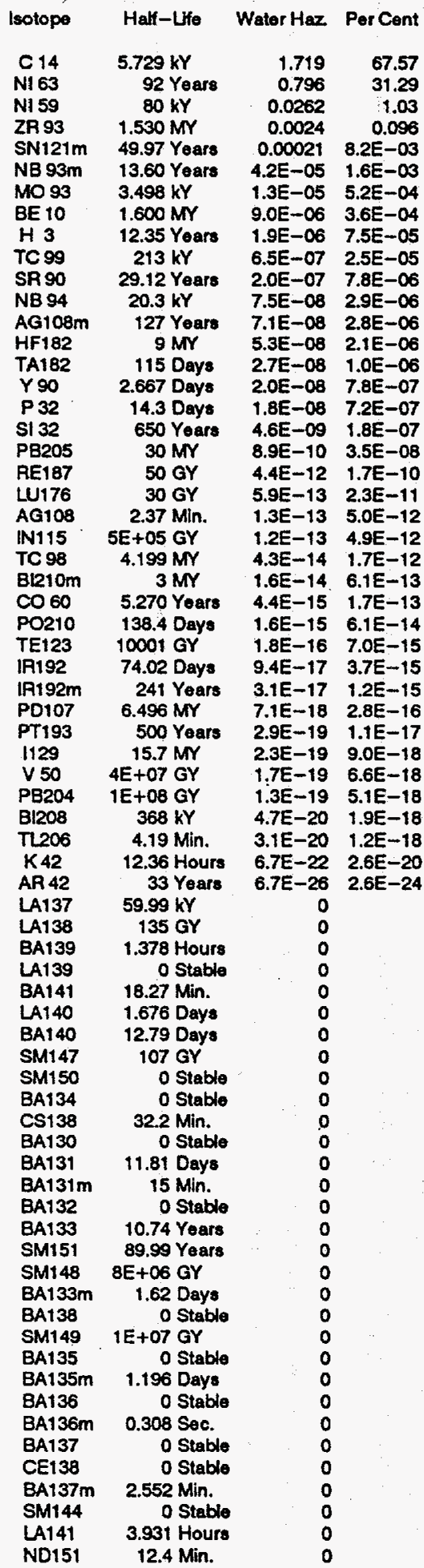

Table 8-H. Decay Time $=1000$ Years

\begin{tabular}{|c|c|c|c|}
\hline lsotope & Half-Life & Water Hez. & PerCent \\
\hline $\begin{array}{r}C 14 \\
50\end{array}$ & $5.729 \mathrm{kY}$ & 1.579 & 97.97 \\
\hline $\begin{array}{l}\text { NI } 59 \\
\text { NI } 63\end{array}$ & $\begin{array}{l}80 \mathrm{kY} \\
92 \text { Years }\end{array}$ & $\begin{array}{l}0.0261 \\
0.0041\end{array}$ & $\begin{array}{r}1.62 \\
0.253\end{array}$ \\
\hline ZF 93 & $1.530 \mathrm{Mr}$ & 0.0024 & 0.151 \\
\hline $\mathrm{NB} 93 \mathrm{~m}$ & 13.60 Years & 4.2E-05 & $2.6 \mathrm{E}-03$ \\
\hline MO 93 & $3.498 \mathrm{kY}$ & $1.2 E-05$ & $7.1 E-04$ \\
\hline BE 10 & $1.600 \mathrm{MY}$ & $9.0 E-06$ & $5.6 \mathrm{E}-04$ \\
\hline TC 99 & $213 \mathrm{kY}$ & $6.5 \mathrm{E}-07$ & 4.0E-05 \\
\hline NB:94 & $20.3 \mathrm{kY}$ & 7.3E-08 & $4.5 E-06$ \\
\hline HF182 & $9 \mathrm{MN}$ & 5.3E-08 & 3.3E-06 \\
\hline TA182 & 115 Days & 2.7E-08 & $1.7 E-06$ \\
\hline SN121m & 49.97 Years & 1.3E-08 & 7.9E-07 \\
\hline $\begin{array}{l}\text { P } 32 \\
\text { S1 } 32\end{array}$ & $\begin{array}{l}14.3 \text { Days } \\
650 \text { Yeare }\end{array}$ & $\begin{array}{l}\text { B.7E-09 } \\
2.2 E-09\end{array}$ & $\begin{array}{l}5.4 E-07 \\
1.4 E-07\end{array}$ \\
\hline $\begin{array}{l}\text { S1 } 32 \\
\text { AGt08m }\end{array}$ & 127 Years & $1.6 E-09$ & $\begin{array}{l}1.4 E-07 \\
9.6 E-08\end{array}$ \\
\hline PB2O5 & $30 \mathrm{MY}$ & $8.9 E-10$ & $5.5 E-08$ \\
\hline RE187 & $50 \mathrm{GY}$ & $4.4 E-12$ & $2.7 E-10$ \\
\hline U176 & $30 \mathrm{GY}$ & $5.9 E-13$ & $3.6 E-11$ \\
\hline IN115 & $5 E+05 \mathrm{GY}$ & $1.2 E-13$ & $7.7 E-12$ \\
\hline TC 98 & $4.199 \mathrm{MY}$ & 4.3E-14 & $2.7 E-12$ \\
\hline $\mathrm{Bl} 210 \mathrm{~m}$ & $3 \mathrm{MY}$ & $1.6 E-14$ & $9.7 E-13$ \\
\hline SP 90 & 29.12 Years & $1.1 E-14$ & $7.1 E-13$ \\
\hline AG108 & 2.37 Min. & $2.8 E-15$ & $1.7 E-13$ \\
\hline PO210 & 138.4 Days & $1.6 E-15$ & $9.7 E-14$ \\
\hline Y 90 & 2.667 Days & $1.1 E-15$ & $7.1 E-14$ \\
\hline TE123 & $10001 \mathrm{GY}$ & $1.8 E-16$ & $1.1 E-14$ \\
\hline IR192 & 74.02 Days & $1.4 E-17$ & $9.0 E-16$ \\
\hline PDi07 & $6.496 \mathrm{MY}$ & $7.1 E-18$ & $4.4 E-16$ \\
\hline IA192m & 241 Years & $4.8 E-18$ & $3.0 E-16$ \\
\hline 1129 & $15.7 \mathrm{Mr}$ & $2.3 E-19$ & $1.4 E-17$ \\
\hline V 50 & 4E+07 GY & $1.7 E-19$ & 1.0E-17 \\
\hline PT193 & 500 Years & $1.4 E-19$ & $8.9 E-18$ \\
\hline PB204 & $1 E+08 \mathrm{GY}$ & $1.3 E-19$ & $8.1 E-18$ \\
\hline B1208 & $368 \mathrm{kY}$ & $4.7 E-20$ & $2.9 E-1$ \\
\hline TL206 & 4.19 Min. & $3.1 E-20$ & $1.9 E-1$ \\
\hline H 3 & 12.35 Years & $1.6 E-23$ & $1.0 E-2$ \\
\hline$K 42$ & 12.36 Hours & $2.8 E-28$ & $1.7 E-2$ \\
\hline AR 42 & 33 Years & $2.8 E-32$ & 1.7E-3 \\
\hline BA131 & 11.81 Days & 0 & \\
\hline LA137 & $59.99 \mathrm{kY}$ & 0 & \\
\hline LA138 & $135 \mathrm{GY}$ & 0 & \\
\hline SM148 & $8 E+06 \mathrm{GY}$ & 0 & \\
\hline LA139 & 0 Stable & 0 & \\
\hline BA130 & o Stable & 0 & \\
\hline BA140 & 12.79 Days & 0 & \\
\hline $\operatorname{cs} 138$ & $32.2 \mathrm{Min}$. & 0 & \\
\hline LA140 & 1.676 Days & 0 & \\
\hline CS137 & 30 Years & 0 & \\
\hline LA141 & 3.931 Hours & 0 & \\
\hline BA131m & 15 Min. & 0 & \\
\hline SM150 & able & 0 & \\
\hline BÁ141 & 18.27 Min. & 0 & \\
\hline BA137 & O Stabio & 0 & \\
\hline SM149 & $1 E+07 \mathrm{GY}$ & 0 & \\
\hline BA135 & O Stable & 0 & \\
\hline BA135m & 1.196 Days & 0 & \\
\hline BA136 & o Stable & 0 & \\
\hline BA133m & 1.62 Days & 0 & \\
\hline BA134 & O Stable & 0 & \\
\hline BA137m & 2.552 Min. & 0 & \\
\hline BA132 & O Stable & 0 & \\
\hline BA138 & O Stable & 0 & \\
\hline SM151 & 89.99 Years & 0 & \\
\hline BA139 & 1.378 Hours & 0 & \\
\hline BAT33 & 10.74 & 0 & \\
\hline $\mathrm{BA} 136 \mathrm{~m}$ & 0.308 & 0 & \\
\hline SM145 & 340 & 0 & \\
\hline CE136 & able & 0 & \\
\hline PM147 & 2.623 & 0 & \\
\hline PM148 & 5.37 Days & 0 & \\
\hline
\end{tabular}


Table 2.8. (contd)

Table 8-1. Decay $7 m e=3000$ Years

\begin{tabular}{|c|c|c|c|}
\hline Isotope & Halt-Lifo & Water $\mathrm{Haz}$ & Per Cont \\
\hline $\begin{array}{l}\text { C } 14 \\
\text { NI } 59\end{array}$ & $\begin{array}{r}5.729 \mathrm{kY} \\
80 \mathrm{kY}\end{array}$ & $\begin{array}{l}1.240 \\
0.026\end{array}$ & $\begin{array}{r}97.78 \\
2.02\end{array}$ \\
\hline ZR 93 & $1.530 \mathrm{MY}$ & $2.4 E-03$ & 0.191 \\
\hline NB $93 m$ & 13.60 Years & $4.2 E-05$ & $3.3 E-03$ \\
\hline BE 10 & $1.600 \mathrm{Mr}$ & $9.0 E-06$ & $7.1 E-04$ \\
\hline MO 93 & $3.498 \mathrm{kY}$ & 7.7E-06 & 6.1E-04 \\
\hline TC 99 & $213 \mathrm{kY}$ & $6.4 E-07$ & 5.1E-0.5 \\
\hline NB 94 & $20.3 \mathrm{kY}$ & $6.8 E-08$ & $5.4 E-06$ \\
\hline HF182 & $9 M Y$ & $5.3 E-08$ & $4.2 E-06$ \\
\hline TA182 & 115 Days & 2.7E-08 & 2.1E-06 \\
\hline N! 63 & 92 Years & $1.2 E-\infty$ & $9.2 E-08$ \\
\hline P 32 & 14.3 Deys & $1.0 E-09$ & $8.2 E-08$ \\
\hline PB205 & $30 \mathrm{MY}$ & $8.9 E-10$ & $7.0 \mathrm{E}-08$ \\
\hline SI 32 & 650 Yoars & $2.6 E-10$ & $2,0 E-08$ \\
\hline RE187 & $50 \mathrm{GY}$ & $4.4 E-12$ & $3.5 E-10$ \\
\hline LU176 & $Y$ & $5.9 E-13$ & $4.6 E-11$ \\
\hline N115 & $5 E+05 \mathrm{GY}$ & $1.2 E-13$ & $9.8 E-12$ \\
\hline TC 98 & $4.199 \mathrm{Mr}$ & $4.3 E-14$ & $3.4 E-12$ \\
\hline AG108m & 127 Years & $2.8 E-14$ & $2.2 E-12$ \\
\hline $\mathrm{B1210m}$ & $3 \mathrm{MY}$ & $1.6 \mathrm{E}-14$ & $1.2 E-12$ \\
\hline PO210 & 138.4 Days & $1.6 E-15$ & $1.2 E-13$ \\
\hline TE123 & $10001 \mathrm{Gr}$ & $1.8 E-16$ & $1.4 E-14$ \\
\hline PD107 & $6.496 \mathrm{MY}$ & $7.1 E-18$ & $5.6 E-16$ \\
\hline IR192 & 74.02 Days & $2.1 E-18$ & $1.6 E-16$ \\
\hline IR192m & 241 Years & $6.9 E-19$ & $5.4 E-17$ \\
\hline 1129 & $15.7 \mathrm{MY}$ & $2.3 E-19$ & $1.8 E-17$ \\
\hline V 50 & $4 E+07 \mathrm{GY}$ & $1.7 E-19$ & 1.3E -17 \\
\hline PB204 & $1 E+08 \mathrm{GY}$ & $1.3 E-19$ & $1.0 E-17$ \\
\hline AG10B & 2.37 Min. & $5.0 E-20$ & $4.0 E-18$ \\
\hline $\mathrm{B} 1208$ & $368 \mathrm{kY}$ & $4.7 E-20$ & 3.7E-18 \\
\hline PT193 & 500 Years & $3.6 E-20$ & $2.8 E-18$ \\
\hline TL206 & 4.19 Min. & $3.1 E-20$ & $2.4 E-18$ \\
\hline SN121m & 49.97 Years & $1.1 E-20$ & $9.0 E-19$ \\
\hline SR 90 & 29.12 Years & & $1.4 E-33$ \\
\hline BA136 & 0 Stable. & 0 & \\
\hline $\mathrm{BA} 136 \mathrm{~m}$ & 0.308 Sec. & 0 & \\
\hline BA135 & O Stable & 0 & \\
\hline $\mathrm{BA} 135 \mathrm{~m}$ & 1.196 Days & 0 & \\
\hline LA138 & $135 \mathrm{GY}$ & 0 & \\
\hline SM145 & 340 Days & 0 & \\
\hline BA141 & 18.27 Min. & 0 & \\
\hline 137 & $59.99 \mathrm{kY}$ & 0 & \\
\hline BA140 & 12.79 Days & 0 & \\
\hline BA137 & 0 Stable & 0 & \\
\hline BA137m & 2.552 Min. & 0 & \\
\hline BA139 & 1.378 Hours & 0 & \\
\hline SM144 & O Stable & 0 & \\
\hline BA138 & O Stable & 0 & \\
\hline BA133m & 1.62 Days & 0 & \\
\hline BA134 & O Stable & 0 & \\
\hline BA130 & O Stable & 0 & \\
\hline SMI 49 & $\mathrm{E}+07 \mathrm{GY}$ & 0 & \\
\hline CS134m & 2.9 Hours & 0 & \\
\hline CS135 & $2.3 \mathrm{MY}$ & 0 & \\
\hline CS136 & 13.1 Days & 0 & \\
\hline CS137 & 30 Years & 0 & \\
\hline SM150 & 0 Stable & 0 & \\
\hline CS138 & 32.2 Min. & 0 & \\
\hline CE136 & $\begin{array}{l}0 \text { Stable } \\
1074 \text { Yoar }\end{array}$ & $\begin{array}{l}0 \\
0\end{array}$ & \\
\hline $\begin{array}{l}\text { BA133 } \\
\text { BA131 }\end{array}$ & $\begin{array}{l}\text { 10.74 Years } \\
11.81 \text { Days }\end{array}$ & 0 & \\
\hline SM148 & $8 E+06 \mathrm{GY}$ & 0 & \\
\hline SM147 & $107 \mathrm{GY}$ & 0 & \\
\hline BA131m & $15 \mathrm{Min}$. & 0 & \\
\hline SM146 & $70 \mathrm{MY}$ & 0 & \\
\hline BA132 & O Stable & 0 & \\
\hline LA139 & O Stable & 0 & \\
\hline ND151 & 12.4 Min. & 0 & \\
\hline ND142 & O Stable & 0 & \\
\hline ND143 & 0 Stable & 0 & \\
\hline
\end{tabular}


Table 2.9. Relative Ingestion Hazard of Actinides in Hanford Reactor Fuel for Various Decay Times

Table 9-A. Decay Time $=1$ Yoas

\begin{tabular}{|c|c|c|c|}
\hline Isotope & Herf-Lito & Wator Haz & Por Cont \\
\hline AM241 & 432.2 Yoars & 20,750 & 52.59 \\
\hline PU239 & $24.05 \mathrm{kY}$ & 12,220 & 30.97 \\
\hline PU240 & $6.537 \mathrm{kY}$ & 2612 & 6.62 \\
\hline PU241 & 14.4 Years & 2399 & 6.08 \\
\hline PU238 & 87.74 Years & 540 & 1.37 \\
\hline CM242 & 163.2 Days & 363 & 0.921 \\
\hline CM244 & 18.11 Years & 220 & 0.558 \\
\hline NP237 & $2.14 \mathrm{MY}$ & 173 & 0.438 \\
\hline U234 & $244.5 \mathrm{kY}$ & 58 & 0.147 \\
\hline U238 & $4.468 \mathrm{GY}$ & 56 & 0.141 \\
\hline $\mathrm{TH} 234$ & 24.1 Days & 33 & 0.085 \\
\hline AM242m & 152 Years & 17 & 0.044 \\
\hline A.:243 & $7.38 \mathrm{kY}$ & 3.9 & $1.0 E-02$ \\
\hline U235 & $703.8 \mathrm{Mr}$ & 2.9 & $7.2 E-03$ \\
\hline RN220 & 55.6 Soc. & 2.7 & $6.9 E-03$ \\
\hline U236 & $23.41 \mathrm{MY}$ & 1.4 & $3.6 E-03$ \\
\hline $\mathrm{CM}_{243}$ & 28.5 Years & 0.50 & $1.3 E-03$ \\
\hline U237 & 6.75 Days & 0.24 & $6.0 E-04$ \\
\hline TH:231 & 1.063 Days & 0.14 & $3.6 E-04$ \\
\hline PA233 & 27 Days & 0.13 & $3.3 E-04$ \\
\hline PU235 & 2.851 Years & 0.11 & $2.7 E-04$ \\
\hline PU242 & $386.9 \mathrm{kY}$ & 0.093 & $2.4 E-0.4$ \\
\hline PA231 & $32.77 \mathrm{kY}$ & 0.039 & $9.9 E-05$ \\
\hline RA224 & 3.66 Days & 0.020 & $5.1 E-05$ \\
\hline TH228 & 1.913 Years & 0.020 & $5.1 E-05$ \\
\hline U232 & 72 Years & 0.014 & $3.5 E-05$ \\
\hline$T H 230$ & $77 \mathrm{kY}$ & 0.013 & $3.2 E-05$ \\
\hline AM242 & 16.02 Hours & 0.0069 & $1.8 E-05$ \\
\hline PA234. & 6.7 Hours & 0.0062 & $1.6 E-05$ \\
\hline NP239 & 2.355 Days & 0.0032 & $6.0 E-06$ \\
\hline PB212 & 10.64 Hours & 0.0027 & $6.9 E-06$ \\
\hline CIM245 & $8.499 \mathrm{kY}$ & 0.0021 & $5.3 E-06$ \\
\hline$A C 227$ & 21.77 Years & 0.00078 & $2.0 E-06$ \\
\hline PA234m & 1.17 Min. & 0.00033 & $8.5 E-07$ \\
\hline RN222 & 3.824 Days & 0.00033 & $8.4 E-07$ \\
\hline NP238 & 2.117. Days & $8.7 E-05$ & $2.2 E-07$ \\
\hline $\mathrm{B} 1212$ & 1.009 Hours & $8.1 E-05$ & $2.1 E-07$ \\
\hline U233 & $158.5 \mathrm{kY}$ & $7.9 E-05$ & $2.0 E-07$ \\
\hline NP236 & $115 \mathrm{kY}$ & $5.7 E-05$ & $1.4 E-07$ \\
\hline PU237 & 45.6 Days & $4.0 E-05$ & $1.0 E-07$ \\
\hline RA223 & 11.43 Days & $3.9 \mathrm{E}-05$ & $9.9 E-08$ \\
\hline CM246 & $4.731 \mathrm{kY}^{\prime}$ & $1.7 \mathrm{E}-05$ & $4.4 E-08$ \\
\hline RA226 & $1.6 \mathrm{kY}$ & $1.0 E-05$ & $2.5 E-08$ \\
\hline NP235 & 1.084 Years & $9.5 E-06$ & $2.4 E-08$ \\
\hline TH227 & 18.72 Days & $1.9 E-06$ & $4.9 E-09$ \\
\hline TH2299 & $7.339 \mathrm{kr}$ & $1,6 E-\infty 6$ & $4.0 E-09$ \\
\hline PE210 & 22.3 Years & $4.2 E-07$ & $1.1 E-09$ \\
\hline RA225 & 14.8 Days & $1.6 E-07$ & $4.0 E-10$ \\
\hline$A C 225$ & 10 Days & $6.4 E-08$ & $1.6 E-10$ \\
\hline PO210 & 138.4 Days & $6.0 E-08$ & $1.5 E-10$ \\
\hline PB211 & 36.1 Min. & $2.6 E-08$ & $6.6 E-11$ \\
\hline TH232 & $14.05 \mathrm{GY}$ & $9.4 E-09$ & $2.4 E-11$ \\
\hline PO216 & 0.15 Sec. & $8.1 E-\infty 9$ & $2.1 E-11$ \\
\hline FRe23 & 21. $8 \mathrm{Min}$. & $5.4 E-09$ & $1.4 E-11$ \\
\hline PO212 & 3E-07 Sec. & $5.2 E-09$ & $1.3 E-11$ \\
\hline PB214 & $26.8 \mathrm{Min}$. & $5.0 E-09$ & $1.3 E-11$ \\
\hline TL208 & 3.07 Min. & $2.9 E-09$ & $7.4 E-12$ \\
\hline $\mathrm{CM} 241$ & 36 Days & 2.0E-09 & $5.0 E-12$ \\
\hline B1214 & $19.9 \mathrm{Min}$. & $1.7 E-09$ & $4.2 E-12$ \\
\hline PU244 & $82.61 \mathrm{MY}$ & $8.2 E-10$ & $2.7 E-12$ \\
\hline B1210 & 5.012 Days & $6.3 E-10$ & $1.6 E-12$ \\
\hline $\mathrm{B} 1213$ & 45.65 Min. & $3.2 E-10$ & $8.1 E-13$ \\
\hline RADB & 6.7 Years & $2.6 E-10$ & $6.6 E-13$ \\
\hline PB209 & 3.3 Hours & $9.1 E-11$ & $2.3 E-13$ \\
\hline U240 & 14.1 Hours & $8.2 E-11$ & $2.1 E-13$ \\
\hline $\mathrm{B} 1211^{\circ}$ & 2.13 Min. & $7.8 E-12$ & $2.0 E-14$ \\
\hline AN219 & 3.96 Sec. & $7.8 E-12$ & $2.0 E-14$ \\
\hline PO215 & 0.002 Sec. & $7.8 E-12$ & $2.0 E-14$ \\
\hline TL207 & $4.77 \mathrm{Min}$. & $7.8 E-12$ & $2.0 E-14$ \\
\hline CM247 & $15.6 \mathrm{Mr}$ & $2.1 E-12$ & $5.4 E-15$ \\
\hline
\end{tabular}

Tabie 9-B. Docay Time $=3$ Yoars

\begin{tabular}{|c|c|c|c|}
\hline lsotope & HaH-Lifo & Water Hax & Per Cent \\
\hline AM241 & 432.2 Years & 57,300 & 75.98 \\
\hline PU239 & $24.06 \mathrm{kY}$ & 12,220 & 16.20 \\
\hline PU240 & $6.537 \mathrm{kY}$ & 2612 & 3.46 \\
\hline PU241 & 14.4 Yoers & 2179 & 2.89 \\
\hline PU238 & 87.74 Years & 532 & 0.705 \\
\hline CM244 & 18.11 Years & 204 & 0.270 \\
\hline NP237 & $2.14 \mathrm{Mr}$ & 173 & 0.229 \\
\hline U234 & $244.5 \mathrm{kY}$ & 58 & 0.077 \\
\hline U238 & $4,468 \mathrm{GY}$ & 56 & 0.074 \\
\hline TH234 & 24.1 Days & 33 & 0.044 \\
\hline$A M 242 m$ & 152 Yoars & 17 & 0.023 \\
\hline CM242 & 163.2 Days & 17 & $2.2 E-02$ \\
\hline FN220 & 55.6 Sec. & 6.8 & $9.0 E-03$ \\
\hline AM243 & $7.38 \mathrm{kr}$ & 3.9 & $5.2 E-03$ \\
\hline U235 & $703.8 \mathrm{MY}$ & 2.9 & $3.8 E-03$ \\
\hline U236 & $23.41 \mathrm{MY}$ & 1.4 & $1.9 E-03$ \\
\hline $\mathrm{CM} 243$ & 28.5 Yoars & 0.48 & $6.3 E-0.4$ \\
\hline U237 & 6.75 Days & 0.21 & $2.8 E-04$ \\
\hline TH231 & 1.063 Days & 0.14 & $1.9 E-04$ \\
\hline PA233 & 27 Days & 0.13 & $1.7 E-04$ \\
\hline PA231 & $32.77 \mathrm{kY}$ & 0.099 & $1.3 E-04$ \\
\hline PU242 & $386.9 \mathrm{kY}$ & 0.093 & $1.2 E-04$ \\
\hline PU236 & 2.851 Years & 0.066 & $8.7 E-05$ \\
\hline PA224 & 3.66 Days & 0.051 & $6.8 \bar{E}-05$ \\
\hline TH228 & 1.913 Years & 0.051 & $6.8 E-05$ \\
\hline$\pi H 230$ & $\pi \mathrm{kY}$ & 0.034 & $4.5 E-05$ \\
\hline U232 & 72 Years & 0.017 & 2.3E-05 \\
\hline AM242 & 16.02 Hours & 0.0068 & $9.1 E-06$ \\
\hline PB212 & 10.64 Hours & 0.0068 & $9.0 E-06$ \\
\hline PA234 & 6.7 Hours & 0.0062 & 8.2E -06 \\
\hline$A C 227$ & 21.77 Years & 0.0050 & $6.7 E-06$ \\
\hline NP239 & 2.355 Days & 0.0032 & $4.2 E-06$ \\
\hline RN222 & 3.824 Days & 0.0023 & $3.1 E-06$ \\
\hline CM245 & $8.499 \mathrm{kY}$ & 0.0021 & 2.8E-06 \\
\hline PA234m & $1.17 \mathrm{Min}$. & 0.00033 & $4.4 E-07$ \\
\hline RA223 & $\{1.43$ Deys & 0.00025 & 3.3E -07 \\
\hline 81212 & 1.009 Hours & 0.00020 & $2.7 E-07$ \\
\hline U233 & $158.5 \mathrm{kY}$ & $8.8 E-05$ & $1.2 E-07$ \\
\hline NP238 & 2.117 Days & $8.6 E-05$ & $1.1 E-07$ \\
\hline RA226 & $1.6 \mathrm{kY}$ & $7.0 E-05$ & 9.3E-08 \\
\hline NP236 & $115 k Y$ & $5.7 E-05$ & $7.6 \mathrm{E}-08$ \\
\hline CM246 & $4.731 \mathrm{kY}$ & $1.7 E-05$ & $2.3 E-08$ \\
\hline TH227 & 18.72 Days & $1.2 \mathrm{E}-05$ & $1.6 E-08$ \\
\hline PB210 & 22.3 Years & $7.6 \mathrm{E}-06$ & $1.0 E-08$ \\
\hline TH229 & $7.339 \mathrm{kY}$ & $3.6 E-06$ & 4.7E-09 \\
\hline NP235 & 1.084 Years & $2.6 E-06$ & $3.5 E-09$ \\
\hline PO210 & 138.4 Days & $1.8 E-\infty 6$ & $2.4 E-09$ \\
\hline RA225 & 14.8 Days & $3.6 E-07$ & $4.7 E-10$ \\
\hline PE211 & 36.1 Min. & $1.7 E-07$ & $2.2 E-10$ \\
\hline AC2225 & 10 Days & $1.4 E-07$ & $1.9 \mathrm{E}-10$ \\
\hline PB214 & 26.8 Min. & $3.5 E-08$ & $4.6 E-11$ \\
\hline FP223 & 21.8 Min. & $3.5 E-08$ & $4.6 E-11$ \\
\hline $\begin{array}{l}\text { TH232 } \\
\text { PO216 }\end{array}$ & $\begin{array}{l}14.05 \mathrm{GY} \\
0.15 \text { Sec. }\end{array}$ & $2.6 \mathrm{E}-08$ & $\begin{array}{l}3.5 E-11 \\
2.7 E-11\end{array}$ \\
\hline $\begin{array}{l}\text { PO216 } \\
\text { PO212 }\end{array}$ & $\begin{array}{r}0.15 \text { Sec. } \\
3 E-07 \text { Sec. }\end{array}$ & $\begin{array}{l}2.0 E-08 \\
1.3 E-08\end{array}$ & $\begin{array}{l}2.7 E-11 \\
1.7 E-11\end{array}$ \\
\hline $\begin{array}{l}\text { PO212 } \\
\text { B1214 }\end{array}$ & $\begin{array}{r}\text { 3E-07 Sec. } \\
19.9 \mathrm{Min} .\end{array}$ & $\begin{array}{l}1.3 E-08 \\
1.2 E-08\end{array}$ & $\begin{array}{l}1.7 E-11 \\
1.6 E-11\end{array}$ \\
\hline 81210 & 5.012 Days & $1.1 E-08$ & $1.5 E-11$ \\
\hline$T L 208$ & $3.07 \mathrm{Min}$ & $7.3 E-09$ & $9.7 E-12$ \\
\hline PAZ28 & 6.7 Years & $1.9 \mathrm{E}-09$ & $2.5 E-12$ \\
\hline PU244 & $82.61 \mathrm{MY}$ & $8.2 E-10$ & $1.1 E-12$ \\
\hline $\mathrm{Bl} 213$ & $45.65 \mathrm{Min}$. & $7.1 E-10$ & $9.5 E-13$ \\
\hline PU237 & 45.6 Days & $6.0 E-10$ & $7.9 \bar{E}-13$ \\
\hline PB209 & 3.3 Hours & $2.0 E-10$ & $2.7 E-13$ \\
\hline U240 & 14.1 Hours & $8.2 E-11$ & $1.1 E-13$ \\
\hline B1211 & $2.13 \mathrm{Min}$. & $5.0 E-11$. & $6.7 E-14$ \\
\hline RN219 & 3.96 Sec. & $5.0 E-11$ & $6.7 E-14$ \\
\hline PO215 & 0.002 Sec. & $5.0 E-11$ & $6.7 E-14$ \\
\hline TL207 & $4.77 \mathrm{Min}$. & $5.0 E-11$ & $6.6 \mathrm{E}-14$ \\
\hline PO218 & $3.05 \mathrm{Min}$. & $7.0 E-12$ & $9.3 E-15$ \\
\hline $\mathrm{PO} 214$ & 2E-04 Sec. & $7.0 E-12$ & $9.3 E-15$ \\
\hline
\end{tabular}


Table 2.9. (contd)

Table 9-C. Decay Time $=10$ Years

\begin{tabular}{|c|c|c|c|}
\hline olope & Haj-Life & Water & $n$ \\
\hline $\begin{array}{l}\text { AM241 } \\
\text { PU239 }\end{array}$ & $\begin{array}{l}\text { 432.2 Years } \\
24.06 \mathrm{KY}\end{array}$ & $\begin{array}{r}159,900 \\
12,220\end{array}$ & $\begin{array}{r}90.18 \\
6.89\end{array}$ \\
\hline PU240 & $.537 \mathrm{kY}$ & 2610 & 1.47 \\
\hline PU241 & 14.4 Years & 1555 & 0.877 \\
\hline & 87.74 Years & 503 & 0.284 \\
\hline & & 173 & \\
\hline CM244 & ears & 156 & 0.068 \\
\hline U234 & & 58 & 0.033 \\
\hline & & 56 & 0.031 \\
\hline TH234 & 24.1 Days & 33 & 0.019 \\
\hline AM242m & & 17 & $9.4 E-03$ \\
\hline RN220 & & 13 & $7.5 E-03$ \\
\hline AM243 & 7. & 3.9 & $2.2 E-03$ \\
\hline U235 & & 2.9 & \\
\hline U236 & & 1.4 & \\
\hline CM242 & lays & 0.55 & $3.1 E-04$ \\
\hline & & 0.40 & \\
\hline & & 0.31 & \\
\hline U237 & ays & 0.15 & $8.6 E-05$ \\
\hline $\mathrm{TH} 231$ & ays & 0.14 & $8.0 E-05$ \\
\hline & & 0.13 & $7.3 E-05$ \\
\hline TH230 & 7 & 0.11 & $6.0 E-05$ \\
\hline RA224 & ays & 0.10 & $5.6 E-05$ \\
\hline 228 & 1.9 & 0.10 & $5.6 E-05$ \\
\hline PU242 & & 0.0 & 5.2E-05 \\
\hline & ars & & $2.6 E-05$ \\
\hline AN222 & 3.8 & 0.0 & $1.3 E-05$ \\
\hline U232 & ars & 0.0 & $1.2 E-05$ \\
\hline PB212 & urs & 0.0 & $7.5 E-00$ \\
\hline PU236 & Years & 0.0 & $6.8 E-06$ \\
\hline AM242 & ours & & $3.7 E-06$ \\
\hline PA234 & & & $3.5 E-06$ \\
\hline NP239 & 2.35 & 0.0 & $1.8 E-06$ \\
\hline & 11. & & $1.3 E-06$ \\
\hline 1245 & 8.49 & 0.00 & $1.2 \mathrm{E}-06$ \\
\hline 226 & & & $4.0 E-07$ \\
\hline $\mathrm{B} 1212$ & ours & 0.0 & 2.3E-07 \\
\hline $\mathrm{PA234m}$ & & & $1.9 E-07$ \\
\hline PB210 & & 0.0 & 1.3E-07 \\
\hline U233 & 158 & 0.0 & $6.8 E-08$ \\
\hline TH 227 & ays & 011 & $6.4 E-08$ \\
\hline PO210 & ays & -05 & $4.9 E-08$ \\
\hline NP238 & 117 Days & -05 & $4.7 E-08$ \\
\hline NP236 & & -05 & $3.2 E-08$ \\
\hline $\mathrm{CM} 246$ & & -05 & $9.8 E-09$ \\
\hline & 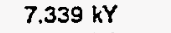 & $1.2 E-05$ & $E-09$ \\
\hline PB211 & $36.1 \mathrm{Min}$. & $1.5 E-06$ & $8.6 E-10$ \\
\hline & bays & $1.2 E-06$ & $E-10$ \\
\hline$A C 225$ & 10 Days & $4.9 E-07$ & $2.7 E-10$ \\
\hline PB214 & 26.8 Min. & $3.5 E-07$ & $2.0 E-10$ \\
\hline & 5.01 & & $2.0 E-10$ \\
\hline FR223 & 21.8 Min. & $3.2 E-07$ & $1.8 \varepsilon-10$ \\
\hline & & $1.2 E-07$ & $6.7 E-11$ \\
\hline TH232 & 14.05 & $8.6 E-08$ & $E-11$ \\
\hline & & $.0 E-08$ & $=-11$ \\
\hline NP235 & 1.08 & $3.0 E-08$ & $1.7 E-11$ \\
\hline & 3E-07 Sec. & $2.6 \mathrm{E}-08$ & $1.4 E-11$ \\
\hline & & & \\
\hline 11208 & 3.07 Min. & $.4 E-08$ & $8.1 E-12$ \\
\hline$B 1213$ & 45.65 Min. & $.4 E-09$ & $1.4 E-12$ \\
\hline & & $8.2 E-10$ & $4.6 E-13$ \\
\hline PB209 & 3.3 Houts & $7.0 E-10$ & $3.9 E-13$ \\
\hline & 2.13 Min. & $4.6 E-10$ & $2.6 E-13$ \\
\hline & 3.96 Sec. & $4.6 E-10$ & $2.6 E-13$ \\
\hline PO215 & 0.002 Sec. & $4.6 E-10$ & $2.6 E-13$ \\
\hline & $4.77 \mathrm{Min}$. & & $2.6 E-13$ \\
\hline U240 & 14.1 Hours & $8.2 E-11$ & $4.6 E-14$ \\
\hline & - & $7.1 E-11$ & $4.0 E-14$ \\
\hline & - n.e & $7.1 E-11$ & \\
\hline & & & \\
\hline
\end{tabular}

Table 9-D. Decay Time $=30$ Yoars

\begin{tabular}{|c|c|c|c|}
\hline lsotopo & Hart-Lifo & Water Haz. & PerCent \\
\hline AM241 & 432.2 Yoars & 312,000 & 95.04 \\
\hline PU239 & $\begin{array}{l}24.06 \mathrm{kY} \\
6.537 \mathrm{kY}\end{array}$ & $\begin{array}{r}12,210 \\
2604\end{array}$ & $\begin{array}{r}3.72 \\
0.793\end{array}$ \\
\hline PU240 & $6.537 \mathrm{kY}$ & $\begin{array}{r}2604 \\
594\end{array}$ & $\begin{array}{l}0.793 \\
0.181\end{array}$ \\
\hline PU241. & 14.4 Years & $\begin{array}{l}594 \\
429\end{array}$ & 0.181 \\
\hline PU238 & 87.74 Yoars & 429 & $\begin{array}{r}0.131 \\
58-0 ?\end{array}$ \\
\hline NP237 & $2.14 \mathrm{Mr}$ & 175 & 5.3E-02 \\
\hline CM244 & 18.11 Years & 73 & $2.2 E-02$ \\
\hline U234 & $244.5 \mathrm{kY}$ & 58 & $1.8 \mathrm{E}-02$ \\
\hline U238 & $4.468 \mathrm{GY}$ & 56 & 1.7E-02 \\
\hline TH234 & 24.1 Days & 33 & $1.0 \mathrm{E}-02$ \\
\hline AM242m & 152 Yoars & 15 & $4.6 \mathrm{E}-03$ \\
\hline RNZ2O & 55.6 Sec. & 13 & $3.9 E-03$ \\
\hline AM243 & $7.38 \mathrm{kY}$ & 3.9 & $1.2 E-03$ \\
\hline U235 & $703.8 \mathrm{Mr}$ & 2.9 & 8.7E-04 \\
\hline U236 & $23.41 \mathrm{MY}$ & 1.4 & 4.4E-04 \\
\hline PA231 & $32.77 \mathrm{kY}$ & 0.91 & $2.8 E-04$ \\
\hline CM242 & 163.2 Days & 0.50 & $1.5 E-04$ \\
\hline AC227 & 21.77 Years & 0.33 & $1.0 E-04$ \\
\hline$\pi+230$ & $77 \mathrm{kY}$ & 0.32 & $9.6 E-05$ \\
\hline CM243 & 28.5 Years & 0.25 & $7.6 E-05$ \\
\hline RN2222 & 3.824 Days & 0.21 & 6.3E-05 \\
\hline TH231 & 1.063 Days & 0.14 & $4.3 E-05$ \\
\hline PA233 & 27 Days & 0.13 & $4.0 E-05$ \\
\hline RA224 & 3.66 Days & 0.096 & $2.9 E-05$ \\
\hline TH228 & 1.913 Yoars & 0.096 & $2.9 E-05$ \\
\hline PU242 & $386.9 \mathrm{kr}$ & 0.093 & 2.8E-05 \\
\hline U237 & 6.75 Days & 0.058 & $1.8 E-05$ \\
\hline U232 & 72 Years & 0.019 & $5.7 E-06$ \\
\hline $\mathrm{F} A 223$ & 11.43 Days & 0.016 & $5.0 E-06$ \\
\hline P8212 & 10.64 Hours & 0.013 & $3.9 E-06$ \\
\hline PA234 & 6.7 Hours & 0.0062 & $1.9 E-06$ \\
\hline RA226 & $1.6 \mathrm{kY}$ & 0.0062 & $1.9 E-06$ \\
\hline AM242 & 16.02 Hours & 0.0061 & $1.8 E-06$ \\
\hline PB210 & 22.3 Years & 0.0052 & $1.6 \mathrm{E}-06$ \\
\hline NP239 & 2.355 Days & 0.0031 & $9.6 E-07$ \\
\hline CM245 & $8.499 \mathrm{kY}$ & 0.0021 & $6.3 E-07$ \\
\hline PO210 & 138.4 Days & 0.0019 & $5.9 E-07$ \\
\hline TH227 & 18.72 Days & 0.00081 & $2.5 E-07$ \\
\hline 81212 & 1.009 Hours & 0.00038 & $1.2 E-07$ \\
\hline PA234m & 1.17 Min. & 0.00033 & $1.0 E-07$ \\
\hline U233 & $158.5 \mathrm{kY}$ & 0.00021 & $6.4 E-08$ \\
\hline PU236 & 2.851 Years & $9.3 E-05$ & $2.8 E-08$ \\
\hline NP238 & 2.117 Days & $7.6 E-05$ & $2.3 E-08$ \\
\hline NP236 & $115 \mathrm{kY}$ & $5.7 E-05$ & $1.7 E-08$ \\
\hline TH229 & $7.339 \mathrm{kY}$ & $5.1 E-05$ & $1.6 E-08$ \\
\hline CM246 & $4.731 \mathrm{kY}$ & $1.7 E-05$ & 5.3E-09 \\
\hline PB211 & 36.1 Min. & $1.1 E-05$ & $3.3 E-09$ \\
\hline 81210 & 5.012 Days & $7.8 E-06$ & $2.4 E-09$ \\
\hline FA225 & 14.8 Days & $5.1 E-06$ & $1.6 \mathrm{E}-09$ \\
\hline PB214 & 26.8 Min. & $3.1 E-06$ & $9.4 E-10$ \\
\hline$F R 223$ & $21.8 \mathrm{Min}$. & $2.3 E-06$ & $6.9 E-10$ \\
\hline$A C 225$ & 10 Days & $2.1 E-06$ & $6.2 E-10$ \\
\hline $\mathrm{B} \mid 214$ & 19.9 Min. & $1.0 E-06$ & $3.1 E-10$ \\
\hline TH232 & $14.05 \mathrm{GY}$ & $2.6 E-07$ & $7.8 E-11$ \\
\hline RA228 & 6.7 Years & $8.9 E-08$ & $2.7 E-11$ \\
\hline PO216 & 0.15 Sec. & $3.8 \mathrm{E}-0.8$ & $1.2 E-11$ \\
\hline PO212 & 3E-07 Sec. & $2.5 E-08$ & $7.5 E-12$ \\
\hline TL208 & 3.07 Min. & $1.4 E-08$ & $4.2 E-12$ \\
\hline $\mathrm{B} 1213$ & 45.65 Min. & $1.0 E-08$ & $3.1 E-12$ \\
\hline $\mathrm{B} 1211$ & $2.13 \mathrm{Min}$. & $3.3 E-09$ & $1.0 E-12$ \\
\hline PO215 & 0.002 Sec. & $3.3 E-09$ & $1.0 E-12$ \\
\hline RN219 & $3.96 \mathrm{Sec}$. & 3.3E-09 & $1.0 E-12$ \\
\hline TL207 & 4.77 Min. & $3.3 E-09$ & $9.9 E-13$ \\
\hline PB209 & 3.3 Hours & 2.9E-O9 & $8.9 E-13$ \\
\hline PU244 & $82.61 \mathrm{Mr}$ & $8.2 E-10$ & $2.5 E-13$ \\
\hline PO218 & $3.05 \mathrm{Min}$. & $6.2 E-10$ & $1.9 E-13$ \\
\hline PO214 & $2 E-04 \mathrm{Sec}$ & $6.2 E-10$ & $1.9 E-13$ \\
\hline$A C 228$ & 6.131 Hours & -10 & $4.5 E-14$ \\
\hline U240 & 14.1 Hours & $8.2 E-11$ & $2.5 E-14$ \\
\hline PO211 & $0.56 \mathrm{Sec}$ & $9.2 E-12$ & $2.8 E-15$ \\
\hline
\end{tabular}


Table 2.9. (contd)

Table 9-E. Decay Time $=40$ Years

\begin{tabular}{|c|c|c|c|}
\hline sotope & aff-Life & Water Haz & Zen \\
\hline $\begin{array}{l}\text { AM241 } \\
\text { PU239 }\end{array}$ & $\begin{array}{l}432.2 \\
24.06\end{array}$ & $\begin{array}{r}344,500 \\
12,210\end{array}$ & $\begin{array}{r}95.57 \\
3.39\end{array}$ \\
\hline PU240 & 6.537 & 2602 & 0.722 \\
\hline & & & 0.110 \\
\hline & & 367 & 0.102 \\
\hline & & 177 & $=-02$ \\
\hline & & 58 & $1.6 E-02$ \\
\hline & & 56 & -02 \\
\hline 1244 & 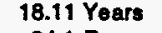 & 49 & $1.4 E-02$ \\
\hline 234 & & 33 & -03 \\
\hline$m$ & & 15 & OE-O3 \\
\hline & & 12 & $2 E-03$ \\
\hline 243 & & 3.9 & $1 E-03$ \\
\hline & & 2.9 & -04 \\
\hline 36 & & 1.4 & $4.0 E-04$ \\
\hline & & 1.2 & $3.4 E-04$ \\
\hline & rars & 0.53 & $1.5 E-04$ \\
\hline 242 & & 0.48 & $1.3 E-04$ \\
\hline 230 & & 0.42 & $1.2 E-04$ \\
\hline RN222 & lays & 0.36 & $1.0 E-04$ \\
\hline 243 & & 0.19 & $5.4 E-05$ \\
\hline 11 & & 0.14 & $4.0 E-05$ \\
\hline 3 & & 0.13 & $3.7 E-05$ \\
\hline 2 & & & -05 \\
\hline 4 & ays & 17 & $2.4 E-05$ \\
\hline 8 & is & 7 & 2.4E-05 \\
\hline U & & 36 & -05 \\
\hline 3 & & 7 & $-\infty$ \\
\hline L & & 7 & -06 \\
\hline 212 & rs & 2 & $-\infty 6$ \\
\hline 210 & ears & 0.011 & $3.2 E-06$ \\
\hline 226 & & 0.011 & 3.0E-06 \\
\hline & ours & 0.0062 & $1.7 E-06$ \\
\hline 242 & ins & 158 & $1.6 E-06$ \\
\hline 210 & & & $1.2 E-06$ \\
\hline 239 & & 0.0031 & 8.7E-07 \\
\hline CM245 & 8. & 0.0021 & $5.8 E-07$ \\
\hline 227 & & & -07 \\
\hline 12 & urs & 335 & $9.7 E-08$ \\
\hline PA234r & & 33 & 9.3 \\
\hline U233 & & & -08 \\
\hline 29 & & 05 & -08 \\
\hline 38 & ays & -05 & -08 \\
\hline 36 & & 5 & $1.6 E-08$ \\
\hline 11 & in. & -05 & $-\infty$ \\
\hline $\mathrm{C}$ & & 25 & -09 \\
\hline 10 & 5. & -05 & $-\infty$ \\
\hline 66 & 8 & 6 & $\begin{array}{l}2.3 E-09 \\
2.2 E-09\end{array}$ \\
\hline R & 14 & $-\infty 6$ & $\begin{array}{l}2.2 E-09 \\
1.5 E-09\end{array}$ \\
\hline $\begin{array}{l}14 \\
23\end{array}$ & & $\begin{array}{l}-\infty 6 \\
-\infty 6\end{array}$ & $\begin{array}{l}1.5 E-09 \\
1.0 E-09\end{array}$ \\
\hline & & -06 & 8.8E-10 \\
\hline 81214 & 19.9 Min. & $1.8 \mathrm{E}-06$ & $5.1 E-10$ \\
\hline TH232 & 14.0 & $3.4 E-07$ & $9.5 E-11$ \\
\hline 228 & 6.7 Years & $1.3 E-07$ & $3.6 E-11$ \\
\hline 216 & & $E-08$ & $9.7 E-12$ \\
\hline & c. & $2.2 E-08$ & $6.2 E-12$ \\
\hline & & -08 & 4.4 \\
\hline & & & 3.5 \\
\hline & & -09 & 1.5 \\
\hline 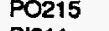 & & & 1.5 \\
\hline & & & 1.5 \\
\hline 207 & & $5.3 E-09$ & $1.5 E-12$ \\
\hline 09 & & 4.5E-09 & $1.3 E-12$ \\
\hline & 2E- & $E-09$ & $3.0 E-13$ \\
\hline . & & $1.1 E-09$ & $3.0 E-13$ \\
\hline & 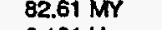 & $8.2 E-10$ & $2.3 E-13$ \\
\hline & 1 Hours & $2.2 E-10$ & 6.08 \\
\hline & un & $8.2 E-11$ & 2. \\
\hline & & & \\
\hline
\end{tabular}

Table 9-F. Decay Time $=100$ Years

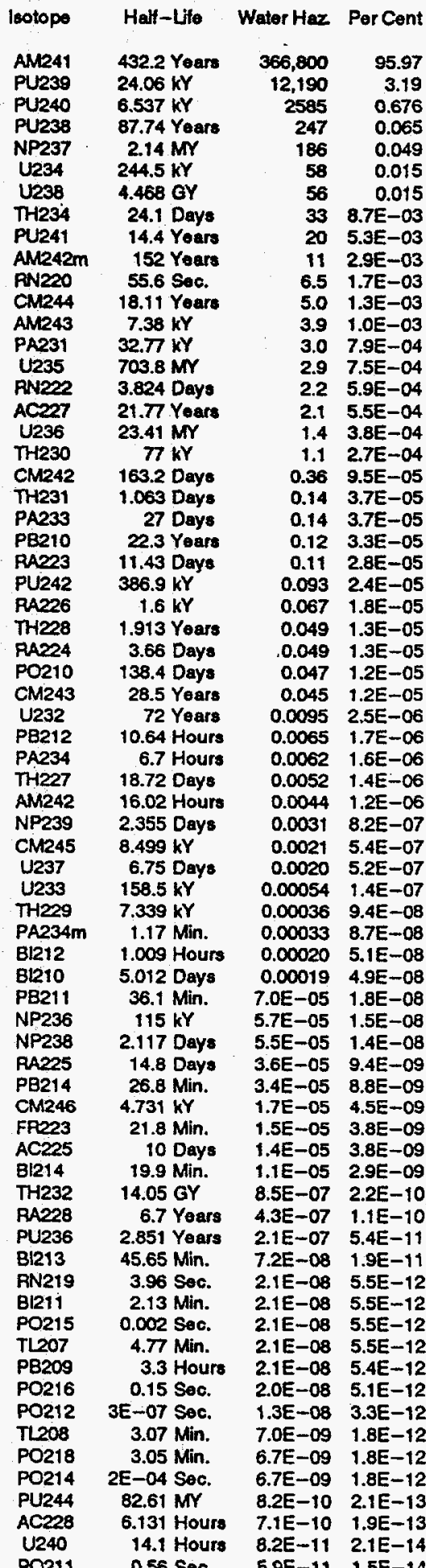


Table 2.9. (contd)

Table 9-G. Docay Time $=300$ Years

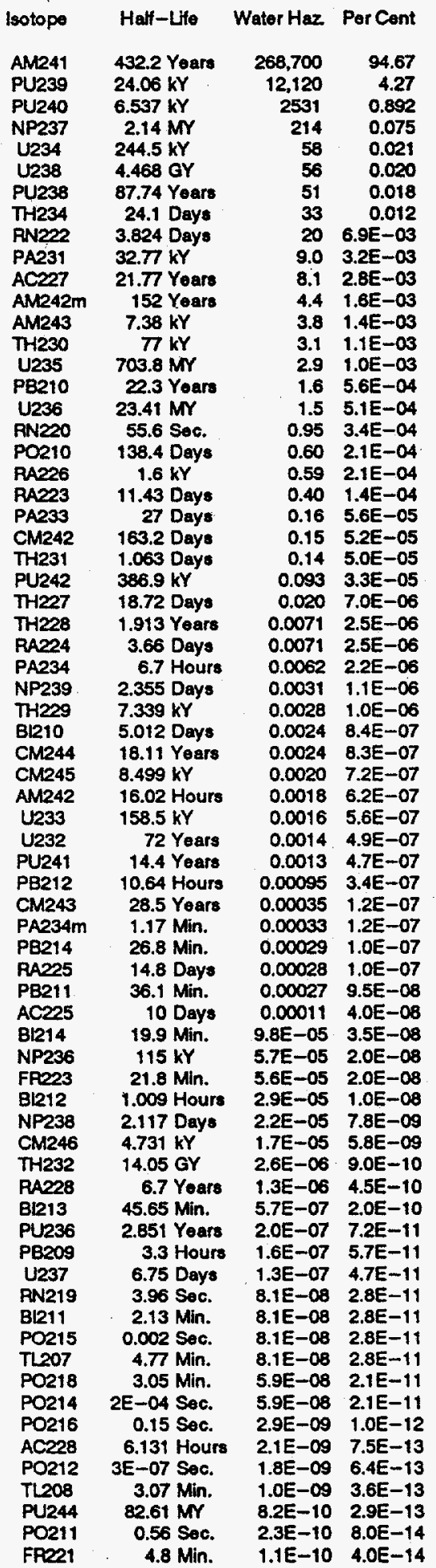

Table 9-H. Decay Time $=1000$ Years

\begin{tabular}{|c|c|c|c|}
\hline lsotope & Half-Lifo & Water Haz. & PerCent \\
\hline AM241 & 432.2 Years & $\begin{array}{l}87,460 \\
11,880\end{array}$ & $\begin{array}{l}85.40 \\
11.60\end{array}$ \\
\hline $\begin{array}{l}\text { PU239 } \\
\text { PU240 }\end{array}$ & $\begin{array}{l}24.06 \mathrm{kY} \\
6.537 \mathrm{kY}\end{array}$ & $\begin{array}{r}11,880 \\
2350\end{array}$ & $\begin{array}{r}11.60 \\
2.29\end{array}$ \\
\hline NP237 & $2.14 \mathrm{Mr}$ & 262 & 0.256 \\
\hline PIN222 & 3.824 Days & 197 & 0.193 \\
\hline U234 & $244.5 \mathrm{kY}$ & 58 & 0.057 \\
\hline U238 & $4.468 \mathrm{GY}$ & 56 & 0.054 \\
\hline TH234 & 24.1 Days & 33 & 0.033 \\
\hline AC227 & 21.77 Yoare & 30 & 0.029 \\
\hline PA231 & $32.77 \mathrm{kY}$ & 30 & 0.029 \\
\hline PB210 & 22.3 Years & 20 & 0.019 \\
\hline TH230 & $\pi \mathrm{kY}$ & 10 & 0.010 \\
\hline PO210 & 138.4 Days & 7.4 & $7.2 E-03$ \\
\hline RA226 & $1.6 \mathrm{KY}$ & 5.9 & $5.8 \mathrm{E}-03$ \\
\hline AM243 & $7.38 \mathrm{kY}$ & 3.6 & $3.5 E-03$ \\
\hline U235 & $703.8 \mathrm{MY}$ & 2.9 & $2.8 E-03$ \\
\hline RA223 & 11.43 Days & 1.5 & $1.5 E-03$ \\
\hline U236 & $23.41 \mathrm{MT}^{\prime}$ & 1.5 & 1.5E-03 \\
\hline PU238 & 87.74 Years & 0.20 & $2.0 \mathrm{E}-04$ \\
\hline PA233 & 27 Days & 0.20 & $1.9 E-04$ \\
\hline AM242m & 152 Yoars & 0.18 & $1.8 E-04$ \\
\hline TH231 & 1.063 Days & 0.14 & $1.4 E-04$ \\
\hline PU242 & $386.9 \mathrm{kY}$ & 0.093 & $9.0 E-05$ \\
\hline TH227 & 18.72 Days & 0.074 & 7.2E-05 \\
\hline TH+229 & $7.339 \mathrm{kY}$ & 0.033 & 3.2E-05 \\
\hline B1210 & 5.012 Days & 0.030 & $2.9 E-05$ \\
\hline PA234 & $\begin{array}{r}6.7 \text { Hours } \\
158.5 \mathrm{kY}\end{array}$ & $\begin{array}{l}0.0062 \\
0.0060\end{array}$ & $6.0 E-06$ \\
\hline U233 & $\begin{array}{l}158.5 \mathrm{KY} \\
163.2 \text { Days }\end{array}$ & $\begin{array}{l}0.0060 \\
0.0060\end{array}$ & $\begin{array}{l}5.9 E-06 \\
5.9 E-06\end{array}$ \\
\hline $\begin{array}{l}\text { CM242 } \\
\text { PA225 }\end{array}$ & $\begin{array}{r}\text { 163.2 Days } \\
\text { 14.8 Days }\end{array}$ & 0.0033 & $\begin{array}{l}5.9 E-06 \\
3.2 E-06\end{array}$ \\
\hline $\begin{array}{l}\text { PA225 } \\
\text { PB214 }\end{array}$ & $\begin{array}{l}\text { 14.8 Days } \\
26.8 \text { Min. }\end{array}$ & 0.0030 & $2.9 E-06$ \\
\hline $\begin{array}{l}\text { PB214 } \\
\text { NP239 }\end{array}$ & & 0.0029 & $2.8 E-06$ \\
\hline CM245 & $8.499 \mathrm{kY}$ & 0.0019 & $1.9 E-\infty 6$ \\
\hline RN220 & $55.6 \mathrm{Sec}$. & 0.0016 & $1.6 \mathrm{E}-06$ \\
\hline AC225 & 10 Days & 0.0013 & 1.3E-06 \\
\hline PB211 & 36.1 Min. & 0.0010 & $9.7 E-07$ \\
\hline B1214 & 19.9 Min. & 0.00099 & $9.6 E-07$ \\
\hline PA234m & & 0.00033 & 3.3E-07 \\
\hline FR223 & 21.8 Min. & 0.00021 & $2.0 E-07$ \\
\hline AM242 & 16.02 Hours & $7.3 E-05$ & $7.1 E-08$ \\
\hline NP236 & $115 \mathrm{kY}$ & $5.7 E-05$ & 5.5E-08 \\
\hline CM246 & $4.731 \mathrm{kY}$ & $1.5 E-05$ & $1.5 E-08$ \\
\hline RA224 & 3.66 Days & 1.2E-05 & $1.2 E-08$ \\
\hline TH228 & 1.913 Yoars & $1.2 E-05$ & $1.2 E-08$ \\
\hline TH232 & $14.05 \mathrm{GY}$ & $8.6 E-06$ & $8.4 E-09$ \\
\hline $\mathrm{Bl} 213$ & $45.65 \mathrm{Min}$. & $6.6 E-\infty 6$ & $6.4 E-09$ \\
\hline RA228 & 6.7 Yoars & $4.3 E-\infty 6$ & $4.2 E-09$ \\
\hline U232 & 72 Years & 2.2E-06 & 2.1E-09 \\
\hline PB209 & 3.3 Hours & $1.9 E-06$ & $1.8 E-09$ \\
\hline P8212 & 10.64 Hours & $1.6 E-06$ & $1.6 \mathrm{E}-09$ \\
\hline NP238 & 2.117 Days & $9.1 E-07$ & $8.9 E-10$ \\
\hline PO218 & 3.05 Min. & $5.9 E-07$ & $5.8 E-10$ \\
\hline PO214 & 2E-04 Sec. & $5.9 E-07$ & $5.8 E-10$ \\
\hline PU241 & 14.4 Years & $3.8 E-07$ & $3.8 E-10$ \\
\hline B1211 & 2.13 Min. & $3.0 E-07$ & 2.9E-10 \\
\hline RN219 & 3.96 Sec. & $3.0 E-07$ & $2.9 E-10$ \\
\hline PO215 & 0.002 Sec. & $3.0 E-07$ & $2.9 E-10$ \\
\hline TL207 & 4.77 Min. & $3.0 E-07$ & $2.9 E-10$ \\
\hline PU236 & 2.851 Years & $2.0 E-07$ & $2.0 E-10$ \\
\hline B1212 & 1.009 Hours & $4.9 \mathrm{E}-08$ & $4.7 E-11$ \\
\hline AC228 & 6.131 Hours & $7.2 \mathrm{E}-09$ & $7.0 E-12$ \\
\hline AT217 & 0.032 Sec. & $1.3 E-09$ & $1.3 E-12$ \\
\hline FR221 & $4.8 \mathrm{Min}$. & $1.3 E-09$ & 1.3E-12 \\
\hline PO213 & $4 E-06$ Sec. & $1.3 E-09$ & $1.3 E-12$ \\
\hline PO211 & 0.56 Sec. & $8.4 E-10$ & $8.2 E-13$ \\
\hline PU244 & & $8.2 E-10$ & $8.0 E-13$ \\
\hline U240 & 14.1 Hours & $8.2 E-11$ & $8.0 E-14$ \\
\hline U237 & 6.75 Days & $3.8 E-11$ & 3.7E-14 \\
\hline TL209 & 2.2 Min. & $2.8 E-11$ & $2.8 E-14$ \\
\hline CM243 & 28.5 Years & $1.4 E-11$ & $1.4 E-14$ \\
\hline
\end{tabular}


Table 2.9. (contd)

Table 9-1. Decay $7 m e=3000$ Years

\begin{tabular}{|c|c|c|c|}
\hline sotope & Hatt-Lifo & Water Haz & Por Cont \\
\hline $\begin{array}{l}\text { PU239 } \\
\text { AM241 }\end{array}$ & $\begin{array}{l}24.06 \mathrm{kY} \\
432.2 \text { Yoara }\end{array}$ & $\begin{array}{r}11,210 \\
3539\end{array}$ & $\begin{array}{l}59.29 \\
18.72\end{array}$ \\
\hline PU240 & $6.537 \mathrm{kY}$ & 1901 & 10.05 \\
\hline PN2202 & 3.824 Days & 1374 & 7.27 \\
\hline NP237 & $2.14 \mathrm{MY}$ & 285 & 1.51 \\
\hline PB210 & 22.3 Yoars & 137 & 0.726 \\
\hline AC227 & 21.77 Yoars & 88 & 0.466 \\
\hline PA231 & $32.77 \mathrm{kY}$ & 88 & 0.466 \\
\hline U234 & $244.5 \mathrm{kY}$ & 58 & 0.308 \\
\hline $\begin{array}{l}\text { U238 } \\
\text { PO210 }\end{array}$ & $\begin{array}{l}4.468 \mathrm{GY} \\
138.4\end{array}$ & $\begin{array}{l}56 \\
51\end{array}$ & $\begin{array}{l}0.294 \\
0.272\end{array}$ \\
\hline $\begin{array}{l}\text { PO210 } \\
\text { PA226 }\end{array}$ & $\begin{array}{l}138.4 \text { Days } \\
1.6 \mathrm{kY}\end{array}$ & $\begin{array}{l}51 \\
41\end{array}$ & $\begin{array}{l}0.272 \\
0.218\end{array}$ \\
\hline TH234 & 24.1 Days & 33 & 0.176 \\
\hline TH230 & $7 \mathrm{kY}$ & 31 & 0.164 \\
\hline RA223 & 11.43 Days & 4.4 & 0.023 \\
\hline AM243 & $7.38 \mathrm{kY}$ & 3.0 & 0.016 \\
\hline U235 & $703.8 \mathrm{MY}$ & 2.9 & 0.015 \\
\hline U236 & $23.41 \mathrm{MY}$ & 1.6 & $8.3 E-03$ \\
\hline TH229 & $7.339 \mathrm{kY}$ & 0.32 & $1.7 E-03$ \\
\hline TH227 & 18.72 Days & 0.22 & $1.1 E-03$ \\
\hline PA233 & 27 Days & 0.21 & $1.1 E-03$ \\
\hline B1210 & 5.012 Days & 0.21 & $1.1 E-03$ \\
\hline TH231 & 1.063 Days & 0.14 & $7.6 E-04$ \\
\hline PU242. & $386.9 \mathrm{kY}$ & 0.092 & $4.9 E-04$ \\
\hline RA225 & 14.8 Days & 0.032 & $1.7 E-04$ \\
\hline PB214 & 26.8 Min. & 0.021 & $1.1 E-04$ \\
\hline U233 & $158.5 \mathrm{kY}$ & 0.021 & 1.1E-04 \\
\hline AC225 & 10 Days. & 0.013 & 6.7E-05 \\
\hline B1214 & $19.9 \mathrm{Min}$. & 0.0069 & $3.6 \mathrm{E}-05$ \\
\hline PA234 & 6.7 Hours & 0.0062 & 3.3E-05 \\
\hline PB211 & 36.1 Min. & 0.0029 & 1.6E-05 \\
\hline NP239 & 2.355 Days & 0.0024 & 1.3E-05 \\
\hline CM245 & $8.499 \mathrm{kY}$ & 0.0016 & $8.6 E-06$ \\
\hline RN220 & 55.6 Soc. & 0.00078 & 4.1E- -06 \\
\hline FR223 & $21.8 \mathrm{Min}$. & 0.00061 & $3.2 E-06$ \\
\hline PA234m & 1.17 Min. & 0.00033 & $1.8 E-06$ \\
\hline B1213 & 45.65 Min. & $6.3 E-05$ & 3.3E-07 \\
\hline NP236 & & $5.6 E-05$ & $3.0 E-07$ \\
\hline TH232 & $14.05 \mathrm{GY}$ & 2.7E-05 & $1.4 E-07$ \\
\hline AM242m & 152 Years & $2.0 \mathrm{E}-05$ & $1.1 \mathrm{E}-07$ \\
\hline $\begin{array}{l}\text { PB209 } \\
\text { PA228 }\end{array}$ & $\begin{array}{l}3.3 \text { Hours } \\
6.7 \text { Years }\end{array}$ & $\begin{array}{l}1.8 E-05 \\
1.3 E-05\end{array}$ & $\begin{array}{l}9.6 \mathrm{E}-08 \\
7.1 \mathrm{E}-08\end{array}$ \\
\hline CM246 & $4.731 \mathrm{kY}$ & $\begin{array}{l}1.3 E-05 \\
1.1 E-05\end{array}$ & $5.9 \mathrm{E}-08$ \\
\hline RA2224 & 3.66 Days & $5.9 E-06$ & $3.1 E-08$ \\
\hline THH228 & t.913 Years & $5.9 E-\infty 6$ & $3.1 E-08$ \\
\hline PO21B & 3.05 Min. & $4.1 E-06$ & 2.2E-08 \\
\hline PO214 & 2E-04 Sec. & 4.1E-06 & $2.2 \mathrm{E}-08$ \\
\hline PO215 & 0.002 Soc. & $8.8 E-07$ & $4.7 E-09$ \\
\hline FN219 & 3.96 Soc. & 8.8E-07 & 4.7E-09 \\
\hline 81211 & $2.13 \mathrm{Min}$. & $8.8 E-07$ & 4.7E-09 \\
\hline TL207 & 4.77 Min. & $8.8 E-07$ & $4.6 \mathrm{E}-09$ \\
\hline PB212 & 10.64 Hours & $7.8 E-07$ & $4.1 E-09$ \\
\hline CM242 & 163.2 Days & $6.6 \mathrm{E}-07$ & $3.5 E-\infty 9$ \\
\hline บ232 & 72 Years & 5.0E-07 & 2.7E-09 \\
\hline PU238 & 87.74 Years & $3.4 E-07$ & $1.8 E-09$ \\
\hline PU241 & 14.4 Yeare & $3.3 E-07$ & $1.7 E-\infty 9$ \\
\hline PU236 & 2.851 Years & $2.0 E-07$ & $1.1 E-\infty$ \\
\hline B1212 & 1.009 Hours & 2.3E-08 & $1.2 \mathrm{E}-10$ \\
\hline AC228 & 6.131 Hours & $2.2 E-08$ & $1.2 E-10$ \\
\hline AT217 & 0.032 Sec. & $1.3 \mathrm{E}-0.8$ & $6.7 E-11$ \\
\hline FP221 & 4.8 Min. & $1.3 E-08$ & $6.7 E-11$ \\
\hline PO213 & 4E-D6 Soc. & $1.2 E-08$ & $6.5 E-11$ \\
\hline AM242 & 16.02 Hours & $8.0 E-09$ & $4.2 E-11$ \\
\hline PO211 & $0.56 \mathrm{~s}$ & 2.5E-09 & $1.3 E-11$ \\
\hline PU244 & $82.61 \mathrm{MY}$ & $8.2 E-10$ & 4.3E-12 \\
\hline 7209 & $2.2 \mathrm{Min}$ & 2.7E-10 & $1.4 E-12$ \\
\hline NP238 & 2.117 Days & 1.0E-to & $5.3 \mathrm{E}-13$ \\
\hline U240 & 14.1 Hours & B.2E-11 & $4.3 E-13$ \\
\hline U237 & 6.75 Days & $3.2 \mathrm{E}-11$ & $1.7 E-13$ \\
\hline PO216 & 0.15 Sec. & $2.3 E-12$ & $1.2 E-14$ \\
\hline
\end{tabular}


Table 2.10. Relative Radiation Hazard of Surface-Deposited Fission Products from Hanford Reactor Fuel for Various Decay Times

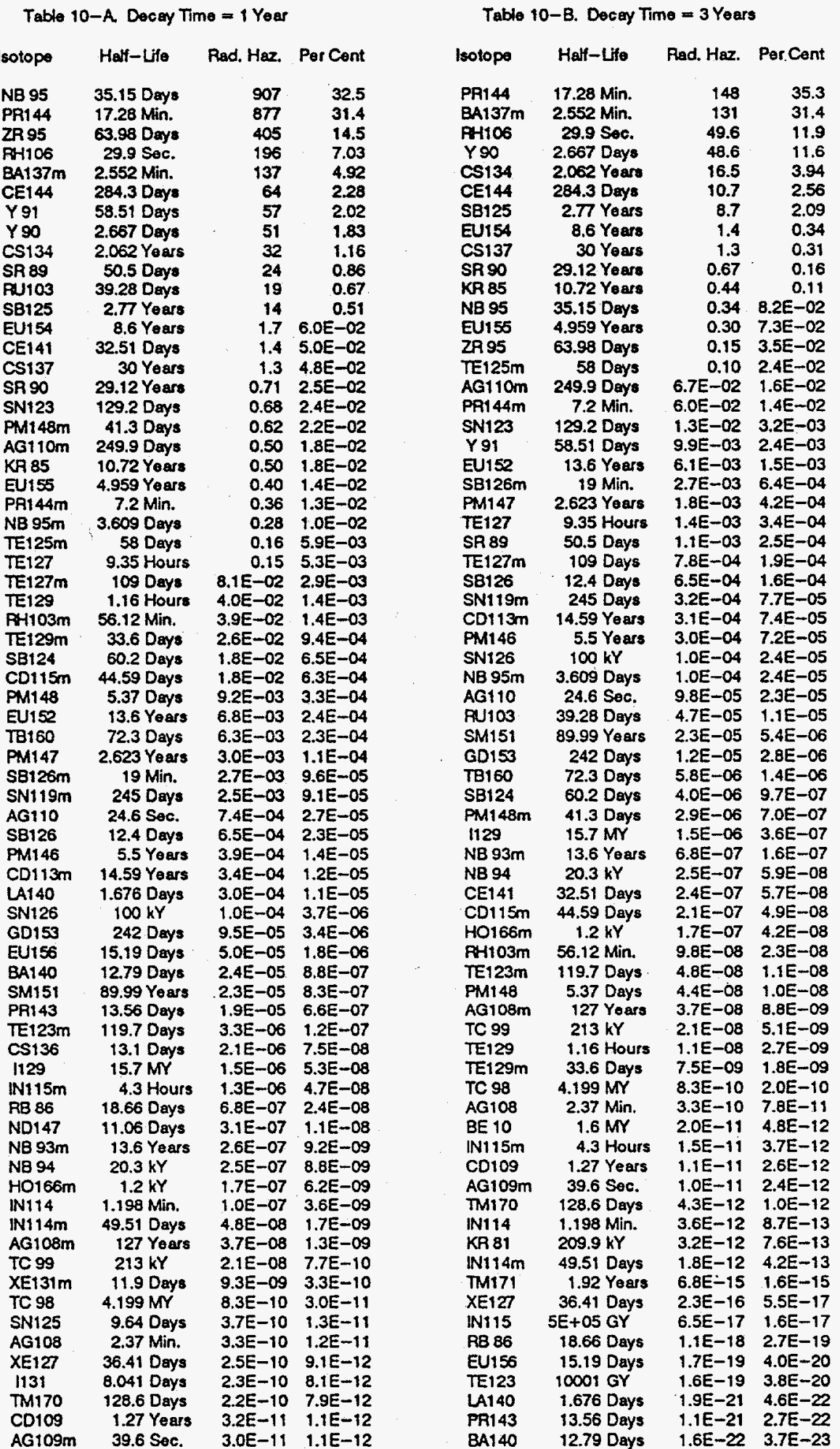


Table 2.10. (contd)

Table 10-C. Decay Time $=10$ Years

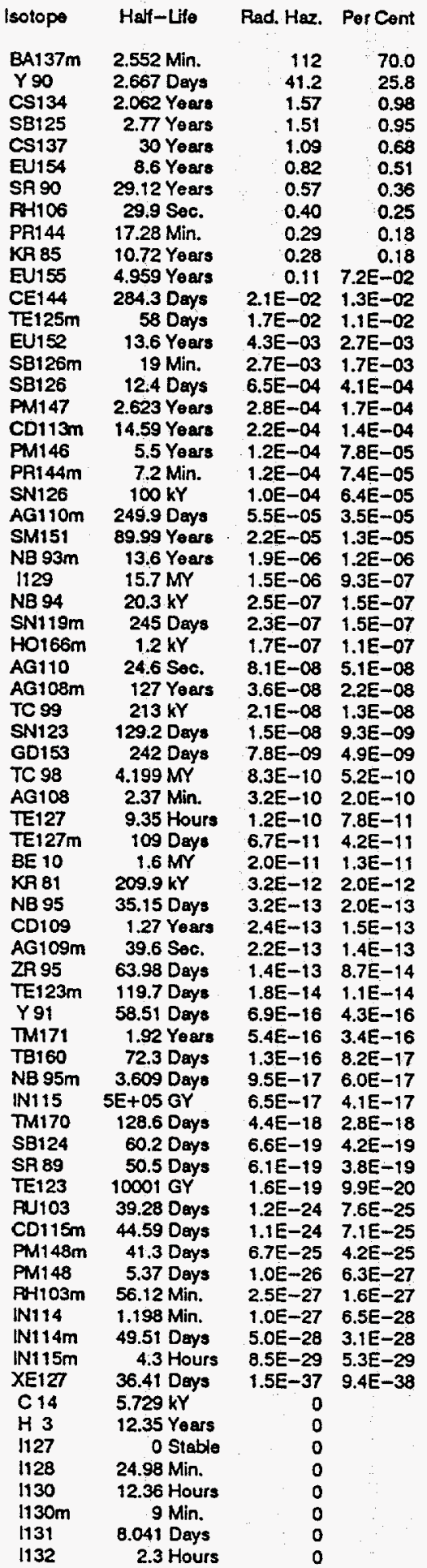

Table 10-D. Docay Time $=30$ Years

\begin{tabular}{|c|c|c|c|}
\hline tope & Half-Lifo & Rad. Haz. & Por Cont \\
\hline $90197 m$ & 2.5521 & 70.3 & \\
\hline & 2.6670 & 25.6 & \\
\hline 3137 & 30 Yoar & 0.68 & 0.7 \\
\hline $\begin{array}{l}\text { SR } 90 \\
\text { EU154 }\end{array}$ & 29.12 Years & 0.35 & $\begin{array}{l}0.36 \\
0.17\end{array}$ \\
\hline $\begin{array}{l}\text { EU154 } \\
\text { KR } 85\end{array}$ & 8.6 Yoara & 0.16 & $\begin{array}{r}0.17 \\
8.0 E-02\end{array}$ \\
\hline $\begin{array}{l}\text { KR } 85 \\
\text { SB125 }\end{array}$ & $\begin{array}{l}10.72 \text { Yeare } \\
2.77 \text { Years }\end{array}$ & $\begin{array}{l}7.7 E-02 \\
1.0 E-02\end{array}$ & $\begin{array}{l}8.0 E-02 \\
1.0 E-02\end{array}$ \\
\hline EU155 & 4.959 Years & $7.0 \mathrm{E}-0.3$ & $7.2 E-03$ \\
\hline $\mathrm{SB126m}$ & $19 \mathrm{Min}$. & $2.7 \mathrm{E}-03$ & $2.7 E-03$ \\
\hline CS134 & $2.062 \mathrm{Y}$ & $1.9 E-03$ & $1.9 E-03$ \\
\hline EU152 & 13.6 Years & $1.6 E-03$ & $1.6 E-03$ \\
\hline SB126 & 12.4 Days & $6.5 E-04$ & 6.7E-04 \\
\hline TE125 & 58 Days & $1.2 E-04$ & $1.2 E-0$ \\
\hline SN126 & $100 \mathrm{kY}$ & $1.0 E-04$ & $1.0 E-0$ \\
\hline CD113m & 14.59 Years & $8.6 E-05$ & $8.8 E-05$ \\
\hline SM151 & 89.99 Years & $1.8 \mathrm{E}-05$ & $1.9 E-05$ \\
\hline PM146 & 5.5 Yoars & $1.0 \mathrm{E}-05$ & $1.0 E-05$ \\
\hline N8 93m & 13.6 Years & $3.6 \mathrm{E}-06$ & $3.7 E-06$ \\
\hline 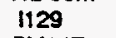 & $15.7 \mathrm{Mr}$ & $1.5 E-06$ & $1.5 E-06$ \\
\hline PM147 & 2.623 Yoars & $1.4 E-06$ & $1.5 E-\infty 6$ \\
\hline PH106 & 29.9 Sec. & 4.3E-07 & $4.4 E-07$ \\
\hline $\begin{array}{l}\text { N8 } 94 \\
\text { HO166m }\end{array}$ & $\begin{array}{r}20.3 \mathrm{kY} \\
1.2 \mathrm{kY}\end{array}$ & $\begin{array}{l}2.5 E-07 \\
1.7 E-07\end{array}$ & $\begin{array}{l}2.5 E-07 \\
1.8 E-07\end{array}$ \\
\hline AG108m & ars & $3.2 E-08$ & 3.3E-08 \\
\hline TC 99 & $213 \mathrm{kY}$ & $2.1 E-08$ & 2.2E-08 \\
\hline PA144 & 17.28 Min. & $5.3 E-09$ & 5.5E-09 \\
\hline $\begin{array}{l}\text { TC } 98 \\
\text { CE144 }\end{array}$ & $4.199 \mathrm{Mr}$ & $\begin{array}{l}\text { 8.3E-10 } \\
3.9 E-10\end{array}$ & $\begin{array}{l}\text { 8.6E-10 } \\
4.0 E-10\end{array}$ \\
\hline $\begin{array}{l}\text { CE144 } \\
\text { AG108 }\end{array}$ & 284.3 Days & & $\begin{array}{l}\text { 4.0E-10 } \\
2.9 E-10\end{array}$ \\
\hline $\begin{array}{l}\text { AG108 } \\
\text { BE 10 }\end{array}$ & $\begin{array}{l}2.37 \mathrm{Min} . \\
1.6 \mathrm{MY}\end{array}$ & $\begin{array}{l}2.8 E-10 \\
2.0 E-11\end{array}$ & $\begin{array}{l}2.9 E-10 \\
2.1 E-11\end{array}$ \\
\hline KA 81 & $209.9 \mathrm{kY}$ & $3.2 E-12$ & 3.3E-12 \\
\hline PRi44m & 7.2 Min. & $2.2 E-12$ & $2.2 E-12$ \\
\hline AG110m & 249.9 Days & $8.8 E-14$ & $9.0 E-14$ \\
\hline SN119m & 245 Days & $2.5 E-16$ & $2.5 E-16$ \\
\hline AG110 & 24.6 Soc. & $1.3 E-16$ & $1.3 E-16$ \\
\hline IN115 & $5 E+05 \mathrm{GY}$ & $6.5 E-17$ & $6.7 \bar{E}-17$ \\
\hline GD153 & Days & $6.4 E-18$ & $6.5 E-18$ \\
\hline CD109 & 1.27 Years & $4.3 E-18$ & $4.4 E-18$ \\
\hline AG109m & 39.6 Sec. & $4.1 E-18$ & $4.2 E-18$ \\
\hline $\mathrm{MM}$ & Years & $4.0 E-19$ & $4.1 E-19$ \\
\hline TE123 & $10001 \mathrm{GY}$ & $1.6 \mathrm{E}-19$ & $1.6 E-19$ \\
\hline SN123 & 12 & $1.4 E-25$ & $1.4 E-25$ \\
\hline TE127 & 9.35 Hours & $8.4 \mathrm{E}-31$ & $8.6 E-31$ \\
\hline TE127m & 109 Days & $4.5 E-31$ & $4.7 E-31$ \\
\hline TE123m & Days & $7.5 E-33$ & $7.7 \bar{E}-33$ \\
\hline nM170 & 5 Days & $3.5 E-35$ & $3.6 E-35$ \\
\hline C 14 & & 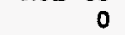 & \\
\hline$H S$ & 12.35 Years & & \\
\hline 1127 & 0 Stable & 0 & \\
\hline & $98 \mathrm{Mi}$ & 0 & \\
\hline 1130 & 12.36 Hours & 0 & \\
\hline $1130 \mathrm{~m}$ & OMi & 0 & \\
\hline 113 & 8.041 Days & 0 & \\
\hline 113 & 2.3 Hours & 0 & \\
\hline 1135 & 20.8 Hours & 0 & \\
\hline $1133 m$ & $9 \mathrm{Se}$ & 0 & \\
\hline & $G M$ & 0 & \\
\hline & $\mathrm{Mi}$ & 0 & \\
\hline 113 & 6.611 Hours & 0 & \\
\hline 1136 & $1.383 \mathrm{Mi}$ & 0 & \\
\hline 1138 & $46 \mathrm{Se}$ & o & \\
\hline 113 & 2 & 0 & \\
\hline 1138 & $6.4 \mathrm{Se}$ & 0 & \\
\hline 113 & $4 \mathrm{So}$ & 0 & \\
\hline 1140 & $0.86 \mathrm{Se}$ & 0 & \\
\hline & $0.4 \mathrm{Se}$ & 0 & \\
\hline 134 & $0.196 \mathrm{Se}$ & 0 & \\
\hline 1143 & $0.328 \mathrm{~S}$ & 0 & \\
\hline & 09320 & 0 & \\
\hline & & & \\
\hline
\end{tabular}


Table 2.10. (contd)

Table 10-E Decay Time $=40$ Years

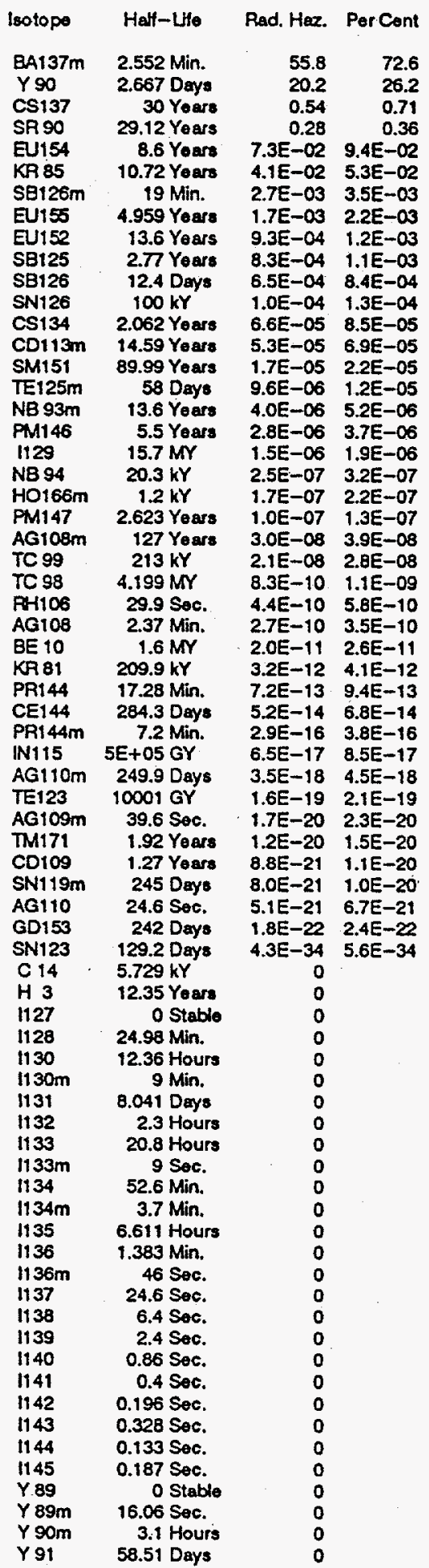

Table to-F. Decay Time $=100$ Years

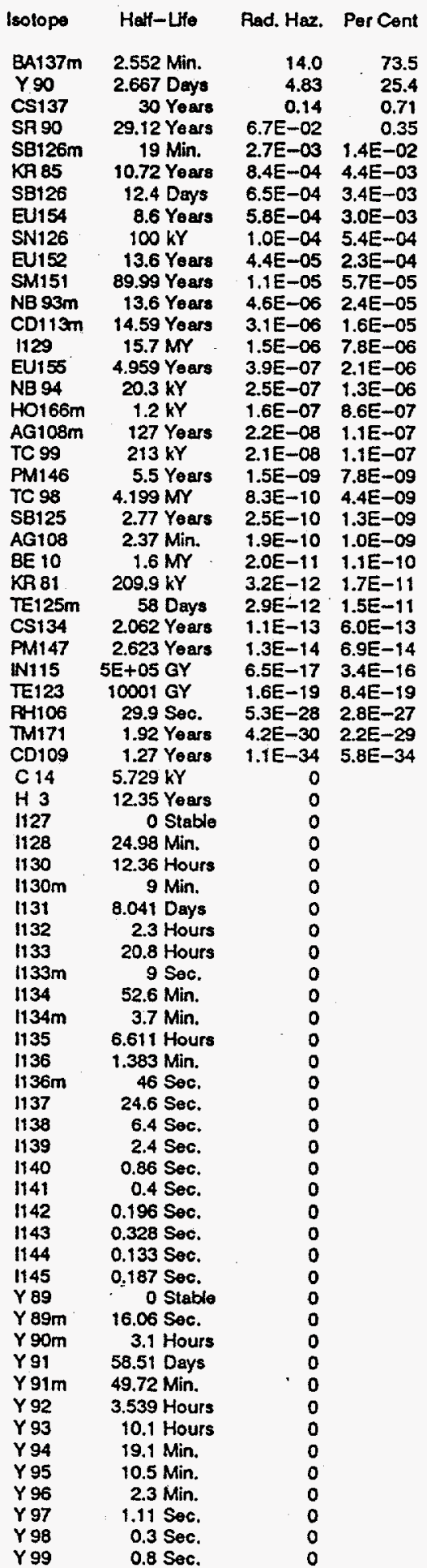


Table 2.10. (contd)

Table 10-Q. Decay Time $=300$ Years

\begin{tabular}{|c|c|c|c|}
\hline Isotope & Haft-Life & Plad. Haz. & Por Cont \\
\hline Mm & 2.552 Min. & $1.4 E-01$ & 74.6 \\
\hline $\begin{array}{l}\text { Y } 90 \\
\text { SB126m }\end{array}$ & $\begin{array}{l}\text { 2.667 Deys } \\
19 \mathrm{Min}\end{array}$ & 4.1E-02 & 22.5 \\
\hline $\begin{array}{l}\text { CS137 } \\
\text { CS13 }\end{array}$ & 30 Years & $\begin{array}{l}2.7 E-0.3 \\
1.3 E-03\end{array}$ & $\begin{array}{l}1.45 \\
0.73\end{array}$ \\
\hline SB126 & 12.4 Days & $6.5 E-04$ & 0.35 \\
\hline SR 90 & 29.12 Yoars & 5.7E-04 & 0.31 \\
\hline & $100 \mathrm{kY}$ & $1.0 E-04$ & $5.5 \mathrm{E}-02$ \\
\hline NB 93m & 13.6 Yoars & 4.6E-06 & $2.5 E-03$ \\
\hline $\begin{array}{l}\text { SiM151 } \\
\text { 1129 }\end{array}$ & 89.99 Yoars & $2.3 E-06$ & $\begin{array}{l}1.3 E-03 \\
8.1 E-0.4\end{array}$ \\
\hline NB 94 & $\begin{array}{l}15.7 \mathrm{MY} \\
20.3 \mathrm{kY}\end{array}$ & $\begin{array}{l}1.5 E-06 \\
2.4 E-07\end{array}$ & 1.3E-04 \\
\hline $\mathrm{HO} 166 \mathrm{~m}$ & $1.2 \mathrm{kY}$ & $1.5 E-07$ & $7.9 E-05$ \\
\hline $\begin{array}{l}\text { TC } 99 \\
\text { AG108m }\end{array}$ & $\begin{array}{l}213 \mathrm{kY} \\
127 \text { Years }\end{array}$ & $\begin{array}{l}2.1 E-08 \\
7.3 E-09\end{array}$ & $\begin{array}{l}1.2 E-05 \\
4.0 E-06\end{array}$ \\
\hline KA 85 & 10.72 Years & $2.0 E-09$ & $\begin{array}{l}4.0 E-06 \\
1.1 E-06\end{array}$ \\
\hline EU152 & 13.6 Years & $1.6 \mathrm{E}-09$ & $8.9 E-07$ \\
\hline TC 98 & $4.199 \mathrm{Mr}$ & 8.3E-10 & $4.5 E-07$ \\
\hline CD113m & 14.59 Years & 2.3E-10 & $1.2 E-07$ \\
\hline AG108 & 2.37 Min. & $6.5 E-11$ & 3.5E-08 \\
\hline EU154 & 8.6 Years & $5.8 E-11$ & $3.1 E-08$ \\
\hline BE 10 & $1.6 \mathrm{Mr}$ & $2.0 E-11$ & $1.1 E-08$ \\
\hline KR 81 & $209.9 \mathrm{kY}$ & $3.2 E-12$ & $1.7 E-09$ \\
\hline IN115 & $5 E+05 \mathrm{GY}$ & $6.5 E-17$ & $3.6 \mathrm{E}-14$ \\
\hline EU155 & 4.959 Yoars & $2.8 E-19$ & 1.5E-16 \\
\hline TE123 & $10001 \mathrm{GY}$ & $1.6 \mathrm{E}-19$ & $8.6 E-17$ \\
\hline $\begin{array}{l}\text { PM146 } \\
\text { C } 14\end{array}$ & $\begin{array}{l}5.5 \text { Years } \\
5.729 \mathrm{kY}\end{array}$ & $\begin{array}{r}1.7 E-20 \\
0\end{array}$ & $9.1 E-18$ \\
\hline $\mathrm{H} 3$ & 12.35 Years & 0 & \\
\hline 1127 & o stablo & 0 & \\
\hline 1128 & 24.98 Min. & 0 & \\
\hline 1130 & 12.36 Hours & 0 & \\
\hline $1130 \mathrm{~m}$ & $9 \mathrm{Min}$. & 0 & \\
\hline 1131 & 8.041 Deys & 0 & \\
\hline 1132 & 2.3 Hours & 0 & \\
\hline 1133 & 20.8 Hours & 0 & \\
\hline $1133 m$ & 9 Soc. & 0 & \\
\hline 1134 & $52.6 \mathrm{Min}$. & 0 & \\
\hline $1134 m$ & 3.7 Min. & 0 & \\
\hline 1135 & 6.611 Hours & 0 & \\
\hline 1136 & 1.383 Min. & 0 & \\
\hline $1136 m$ & 46 Sec. & 0 & \\
\hline 1137 & 24.6 Soc. & 0 & \\
\hline 1138 & 6.4 Soc. & 0 & \\
\hline 1139 & 2.4 Soc. & 0 & \\
\hline 1140 & 0.86 Sec. & 0 & \\
\hline 1141 & 0.4 Soc. & 0 & \\
\hline 1142 & 0.196 Soc. & 0 & \\
\hline 1943 & 0.328 Sec. & 0 & \\
\hline 1144 & 0.133 Sec. & 0 & \\
\hline 1145 & 0.187 Sec. & 0 & \\
\hline Y 89 & O Stable & 0 & \\
\hline Ye9m & 16.06 Sec. & 0 & \\
\hline Y90m & 3.1 Hours & 0 & \\
\hline Y 91 & 58.51 Days & 0 & \\
\hline Y $91 \mathrm{~m}$ & 49.72 Min. & & \\
\hline Y92 & 3.539 Hours & 0 & \\
\hline$Y 93$ & 10.1 Hours & 0 & \\
\hline$Y 94$ & 19.1 Min. & 0 & \\
\hline Y 95 & $10.5 \mathrm{Min}$. & 0 & \\
\hline Y 96 & 2.3 Min. & 0 & \\
\hline Y 97 & 1.11 Soc. & & \\
\hline Y 98 & 0.3 Sec. & 0 & \\
\hline Y99 & 0.8 Sec. & 0 & \\
\hline Y100 & $0.756 \mathrm{Soc}$. & 0 & \\
\hline Y101 & $0.976 \mathrm{Sec}$. & 0 & \\
\hline Y102 & 0.273 Soc. & 0 & \\
\hline Y103 & 0.366 Soc. & 0 & \\
\hline$Y 104$ & 0.144 Soc. & $\begin{array}{l}0 \\
0\end{array}$ & 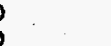 \\
\hline $\begin{array}{l}\text { Y105 } \\
\text { Y106 }\end{array}$ & $0.093 \mathrm{Sec}$ & $\begin{array}{l}0 \\
0\end{array}$ & \\
\hline & & & \\
\hline
\end{tabular}

Table 10-H. Decay Time $=1000$ Years

\begin{tabular}{|c|c|c|c|}
\hline tope & Halt-Lifo & Rad. Hez. & Per Cent \\
\hline $\mathrm{sB126m}$ & $19 \mathrm{Min}$. & $2.7 E-03$ & 77. \\
\hline SB126 & 12.4 Doys & $6.4 E-04$ & 18.9 \\
\hline SN126 & $100 \mathrm{kY}$ & $1.0 E-04$ & 2.97 \\
\hline NB $93 m$ & 13.6 Years & $4.6 E-06$ & 0.14 \\
\hline 1129 & $15.7 \mathrm{Mr}$ & $1.5 E-\infty 6$ & 4.4E-02 \\
\hline NB 94 & $20.3 \mathrm{kY}$ & $2.4 E-07$ & $7.0 E-03$ \\
\hline $\begin{array}{l}\text { HO166m } \\
\text { TC } 99\end{array}$ & $1.2 \mathrm{kY}$ & $9.8 \mathrm{E}-0 \mathrm{~B}$ & $2.9 E-03$ \\
\hline $\begin{array}{l}\text { TC } 99 \\
\text { BA137m }\end{array}$ & $\begin{array}{c}213 \mathrm{KY} \\
2.552 \mathrm{Min} .\end{array}$ & $\begin{array}{l}2.1 E-08 \\
1.3 E-08\end{array}$ & $\begin{array}{l}6.3 E-04 \\
3.8 E-04\end{array}$ \\
\hline SM151 & 89.99 Years & $1.1 E-08$ & $3.1 E-04$ \\
\hline $\begin{array}{l}\text { Y } 90 \\
\text { TC } 98\end{array}$ & 2.667 Deys & $2.4 \bar{E}-09$ & $7.1 \vec{E}-05$ \\
\hline $\begin{array}{l}\text { TC } 98 \\
A G 108 \mathrm{~m}\end{array}$ & $\begin{array}{l}4.199 \mathrm{MY} \\
127 \text { Years }\end{array}$ & $\begin{array}{l}8.3 E-10 \\
1.6 E-10\end{array}$ & $\begin{array}{l}2.4 E-05 \\
4.7 E-06\end{array}$ \\
\hline Csi37 & 30 Years & $1.3 E-10$ & 3.7E-06 \\
\hline $\begin{array}{l}\text { SR } 90 \\
\text { PF } 10\end{array}$ & 29.12 Years & 3.3E-11 & $\begin{array}{l}9.8 E-07 \\
5.9 E-07\end{array}$ \\
\hline $\begin{array}{l}\text { BE } 10 \\
\text { KR } 81\end{array}$ & $\begin{array}{r}1.6 \mathrm{MY} \\
209.9 \mathrm{kY}\end{array}$ & $\begin{array}{l}2.0 E-11 \\
3.2 E-12\end{array}$ & $\begin{array}{l}5.9 E-07 \\
9.3 E-08\end{array}$ \\
\hline AG108 & $2.37 \mathrm{Min}$. & $1.4 E-12$ & $4.2 E-08$ \\
\hline IN1 15 & $5 E+05 \mathrm{GY}$ & $6.5 E-17$ & $1,9 E-12$ \\
\hline TE123 & $10001 \mathrm{GY}$ & $1.6 \mathrm{E}-19$ & 4.7E-15 \\
\hline CD113m & 14.59 Years & 8. $3 E-25$ & $2.4 E-20$ \\
\hline EU152 & 13.6 Years & 5.3E-25 & $1.5 E-20$ \\
\hline $\begin{array}{l}\text { KR } 85 \\
C_{14}\end{array}$ & $\begin{array}{l}\text { 10.72 Years } \\
5.729 \mathrm{kY}\end{array}$ & $\begin{array}{r}4.5 E-29 \\
0\end{array}$ & $1.3 E-24$ \\
\hline $\mathrm{H}_{3}$ & 12.35 Years & 0 & \\
\hline 1127 & O Stable & 0 & \\
\hline $\begin{array}{l}1128 \\
1130\end{array}$ & 24.98 Min. & 0 & \\
\hline $\begin{array}{l}1130 \\
1130 \mathrm{~m}\end{array}$ & $\begin{array}{l}\text { 12.36 Hours } \\
9 \mathrm{Min} .\end{array}$ & $\begin{array}{l}0 \\
0\end{array}$ & \\
\hline 1131 & 8.041 Days & 0 & \\
\hline 1132 & 2.3 Hours & 0 & \\
\hline 1133 & 20.8 Hours & 0 & \\
\hline $1133 m$ & I Sec. & 0 & \\
\hline 1134 & 52.6 Min. & 0 & \\
\hline $1134 \mathrm{~m}$ & 3.7 Min. & 0 & \\
\hline 1135 & 6.611 Hours & 0 & \\
\hline 1136 & $1.383 \mathrm{Min}$ & 0 & \\
\hline $1136 m$ & 46 Soc. & 0 & \\
\hline 1137 & 24.6 Soc. & 0 & \\
\hline 1138 & $6.4 \mathrm{Soc}$. & 0 & \\
\hline 1139 & 2.4 Soc. & 0 & \\
\hline 1140 & 0.86 Sec. & 0 & \\
\hline 1141 & 0.4 Soc. & 0 & \\
\hline 1142 & 0.196 Sec. & 0 & \\
\hline 1143 & 0.328 Sec. & 0 & \\
\hline 1144 & 0.133 Sec. & 0 & \\
\hline 1145 & 0.187 Sec. & 0 & \\
\hline Y 89 & & & \\
\hline Y $89 m$ & 16.06 Sec. & & \\
\hline Y90m & 3.1 Hours & 0 & \\
\hline Y 91 & 58.51 Days & 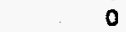 & \\
\hline Y $91 \mathrm{~m}$ & $49.72 \mathrm{Min}$. & 0 & \\
\hline Y92 & 3.539 Hours & 0 & \\
\hline Y 93 & 10.1 Hours & 0 & \\
\hline Y94 & 19.1 Min. & 0 & \\
\hline Y95 & 10.5 Min. & 0 & \\
\hline Y96 & 2.3 Min. & 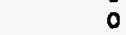 & \\
\hline Y 97 & 1.11 Sec. & 0 & \\
\hline Y 98 & 0.3 Sec. & 0 & \\
\hline Y 99 & 0.8 Sec. & 0 & \\
\hline Y100 & 0.756 Soc. & 0 & \\
\hline Y101 & 0.976 Sec. & 0 & \\
\hline Y102 & 0.273 sec. & 0 & \\
\hline Y103 & 0.366 Sec. & 0 & \\
\hline Y104 & 0.144 Sec. & 0 & \\
\hline Y105 & 0.174 Sec. & 0 & \\
\hline Y106 & 0.093 Soc. & 0 & \\
\hline Y107 & 0.105 Sec. & 0 & \\
\hline AG106 & 8.5 Days & 0 & \\
\hline AG107 & O Stable & 0 & \\
\hline
\end{tabular}


Table 2.10. (contd)

Table 10-1. Decay Time $=3000$ Years

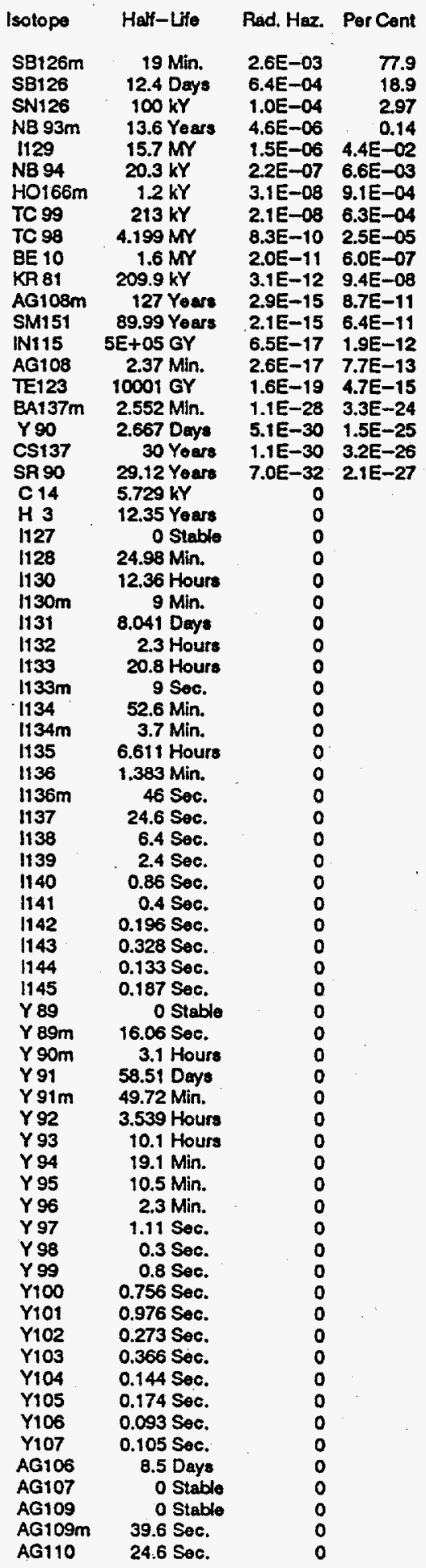


Table 2.11. Relative Radiation Hazard of Surface-Deposited Activation Products from Hanford Reactor Fuel and Cladding at Various Decay Times

Table 11-A. Decay Time $=1$ Year

\begin{tabular}{|c|c|c|c|}
\hline sotope & Helf-Lifo & Rad. Haz. & Per Cent \\
\hline & Dayo & 18 & 39.3 \\
\hline & sars & 0.602 & 21.1 \\
\hline ZRS & Days & 0.498 & 17.5 \\
\hline & Days & 0.382 & 13.4 \\
\hline MN 54 & 312. & 0.106 & 3.72 \\
\hline & ears & 0.093 & 3.26 \\
\hline IN113m & 1.658 Hours & 0.030 & 1.07 \\
\hline SN123 & Days & $5.1 E-03$ & 0.18 \\
\hline $19 \mathrm{~m}$ & Days & $3.8 E-03$ & 0.13 \\
\hline & Days & $1.9 E-03$ & $6.7 E-02$ \\
\hline & Deys & $1.8 E-03$ & $6.5 E-02$ \\
\hline SNI13 & 115.1 Dxys & $1.7 E-03$ & 6.0E-02 \\
\hline TE125m & 58 Days & $1.1 E-03$ & $3.7 E-02$ \\
\hline & 'oars & $5.9 E-04$ & 2.1E-02 \\
\hline & leys & 4.7E-04 & $1.7 E-02$ \\
\hline NE & lays & $3.4 E-04$ & $1.2 E-02$ \\
\hline & ays & $3.1 E-04$ & 1.1E-02 \\
\hline C & Days & $8.8 E-05$ & $3.1 E-03$ \\
\hline & bays & $6.4 E-05$ & $2.2 E-03$ \\
\hline & ays & $6.3 E-05$ & $2.2 E-03$ \\
\hline & ys & $3.5 E-05$ & $1.2 E-03$ \\
\hline c & 4 & $1.7 E-05$ & $6.1 E-04$ \\
\hline $\mathbf{R}$ & urs & $1.1 E-05$ & $3.8 E-04$ \\
\hline v & Jays & -06 & $3.2 E-04$ \\
\hline IN & Min. & $5.6 E-06$ & $2.0 E-04$ \\
\hline 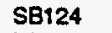 & Days & $5.5 E-06$ & -04 \\
\hline $4 m$ & Days & $2.7 E-06$ & $9.6 E-05$ \\
\hline$\infty$ & lears & 2.7E-06 & $9.6 E-05$ \\
\hline A & & $2.6 E-06$ & 9.1E-05 \\
\hline & & 7.7E-07 & 2.7E-05 \\
\hline & & 9.8E-08 & $3.5 E-06$ \\
\hline $3 m$ & Days & $7.6 E-08$ & $2.7 E-06$ \\
\hline r & & 5.7E- -08 & $2.0 E-06$ \\
\hline A & 24 & 4.9E-08 & $1.7 E-06$ \\
\hline $17 \mathrm{~m}$ & rys & $2.5 E-08$ & $8.8 E-07$ \\
\hline & ys & $6.1 E-09$ & 2.1E-07 \\
\hline $7 n$ & ys & $3.9 E-09$ & $1.4 E-07$ \\
\hline $93 m$ & ars & $1.2 E-09$ & 4.3E-08 \\
\hline A & is & -09 & $4.2 E-08$ \\
\hline$M$ & 3. & $8.1 E-10$ & $2.8 E-08$ \\
\hline 94 & & $=-10$ & $1.3 E-08$ \\
\hline 90 & ears & -11 & -09 \\
\hline 10 & in & -11 & -09 \\
\hline 110 & Sec. & $7.1 E-11$ & -09 \\
\hline & Days & $3.2 E-11$ & -09 \\
\hline 27 & Hours & $1.6 E-11$ & -10 \\
\hline 108 & 2. & -11 & -10 \\
\hline 126 & ys & $1.0 E-11$ & $3.6 \mathrm{E}-10$ \\
\hline & Days & $9.9 E-12$ & 3.5E-10 \\
\hline TE127m & Days & $8.4 E-12$ & $3.0 E-10$ \\
\hline & ys & $2.8 E-12$ & $9.9 E-11$ \\
\hline & & $2.4 E-12$ & $8.4 E-11$ \\
\hline & & -14 & $1.7 E-12$ \\
\hline 205 & & $6.1 E-15$ & $2.1 E-13$ \\
\hline & & -15 & $1.4 E-13$ \\
\hline & & $3.8 E-15$ & $1.3 E-13$ \\
\hline$T$ & & $1.8 E-16$ & $6.5 E-15$ \\
\hline & & -17 & $2.8 E-15$ \\
\hline & ays & -17 & $1.4 E-15$ \\
\hline 08 & 3 & -17 & $3.8 E-16$ \\
\hline & Days & -18 & -16 \\
\hline & Days & -18 & -17 \\
\hline TE & Hours & -21 & $3.1 E-19$ \\
\hline & & -21 & $2.2 E-19$ \\
\hline 123 & 1000 & $3.7 E-21$ & $1.3 E-19$ \\
\hline 106 & Days & $3,6 E-22$ & $1.3 E-20$ \\
\hline PT193 & 500 Yea & $2.2 E-23$ & $7.8 E-22$ \\
\hline & 12.36 & $2.2 E-24$ & $7.8 \mathrm{E}-23$ \\
\hline$A$ & 7.45. Deys & $3.2 E-25$ & $1.1 E-23$ \\
\hline & & $E-25$ & $8.9 E-24$ \\
\hline
\end{tabular}

Table $11-B$. Decay Time $=3$ Years

\begin{tabular}{|c|c|c|c|}
\hline otope & Half-Life & Rad. Haz. & r Cent \\
\hline - & 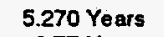 & 0.70 & . \\
\hline & 2.7 & 0.056 & 10. \\
\hline MN 5 & 312.5 Days & 0.021 & 3.86 \\
\hline TE125m & 58 Days & $6.5 E-04$ & 0.12 \\
\hline SN119m & 245 Days & $4.9 E-04$ & $9.0 \mathrm{E}-02$ \\
\hline & 35.15 Days & -04 & $7.7 E-02$ \\
\hline $\mathrm{N} 113 \mathrm{~m}$ & urs & $3.7 E-04$ & $6.9 E-02$ \\
\hline & & $3.5 E-04$ & $6.4 E-02$ \\
\hline & 70. & $E-04$ & $5 E-02$ \\
\hline & 63. & E-O4 & $E-02$ \\
\hline & 125 & $1.0 E-04$ & $1.9 E-02$ \\
\hline SN113 & 115 & $2.1 E-05$ & $3.8 E-03$ \\
\hline & & -06 & $1.5 E-03$ \\
\hline & & & $1.1 E-03$ \\
\hline & ars & -07 & -04 \\
\hline & & & -04 \\
\hline & & -07 & 1 \\
\hline w & 121 & $=-07$ & -05 \\
\hline & ays & -07 & 2 \\
\hline sc & 83. & -08 & 1.5 \\
\hline & & -08 & \\
\hline$F$ & 8 & & $\begin{array}{l}4 \\
2\end{array}$ \\
\hline & 16. & -09 & $1.4 E-06$ \\
\hline On & 24 & -09 & -06 \\
\hline & 2.6 & $-\infty$ & $1.1 E-06$ \\
\hline $3 m$ & 13. & -09 & -07 \\
\hline & & -09 & -07 \\
\hline & & $1.2 E-09$ & 2.3E-07 \\
\hline$A C$ & & $1.2 E-09$ & $2.2 \mathrm{E}-07$ \\
\hline & 115 & -09 & $2.0 \mathrm{E}-07$ \\
\hline & & 10 & -07 \\
\hline & & -10 & -08 \\
\hline & & -10 & -08 \\
\hline In & 1.1 & $=-10$ & $:-08$ \\
\hline 4 & & -10 & -08 \\
\hline In & 49. & $=-11$ & -0 \\
\hline B & 1.6 & $=-11$ & -08 \\
\hline & 29. & $E-11$ & -08 \\
\hline n & is & -11 & -08 \\
\hline & ys & $6.7 E-11$ & -08 \\
\hline$A G 108$ & 2.3 & -11 & -09 \\
\hline & & $9.8 E-12$ & -09 \\
\hline$A G 110$ & Sec. & $9.4 E-12$ & -09 \\
\hline 177 & 6.70 & $1.2 E-12$ & -10 \\
\hline CR 51 & 27. & $1.0 E-12$ & 1.8 \\
\hline TE127 & urs & $1.5 E-13$ & 2.8 \\
\hline TE & & 8.1E-14 & -11 \\
\hline & & $=-15$ & $1.1 \mathrm{E}-12$ \\
\hline & & -15 & -13 \\
\hline & & -16 & 3. \\
\hline & 74 & -17 & 8 \\
\hline & & -17 & 2. \\
\hline R & 35 & -18 & 1. \\
\hline & & -18 & 6 \\
\hline & & -18 & 1. \\
\hline & Ft & -21 & 1. \\
\hline & 190 & 3 & 7. \\
\hline & ears & $2.2 E-23$ & 4.1 \\
\hline & 36. & $6.6 E-24$ & 1.2 \\
\hline SN117m & & $5.0 E-24$ & 9.1 \\
\hline & 12.3 & $2.2 E-24$ & 4.1 \\
\hline 1129 & & $2.5 E-25$ & 4. \\
\hline RH106 & & $1.6 E-25$ & -23 \\
\hline & & 7.0 & 1.3 \\
\hline & 12 & $1.9 E-29$ & -27 \\
\hline & 15. & $1.1 E-32$ & -30 \\
\hline NB 92 & 10. & $1.7 E-36$ & $3.2 E-34$ \\
\hline & & & \\
\hline & & & \\
\hline
\end{tabular}


Table 2.11. (contd)

Table $11-$ C. Decay Time $=10$ Years

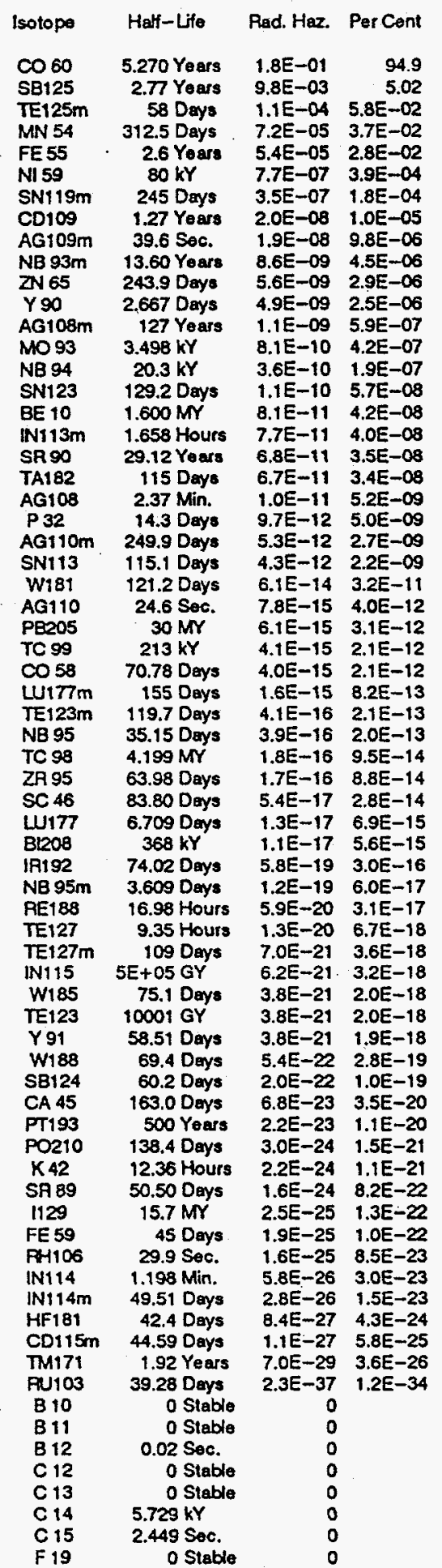

Table 11-D. Decay Time $=30$ Years

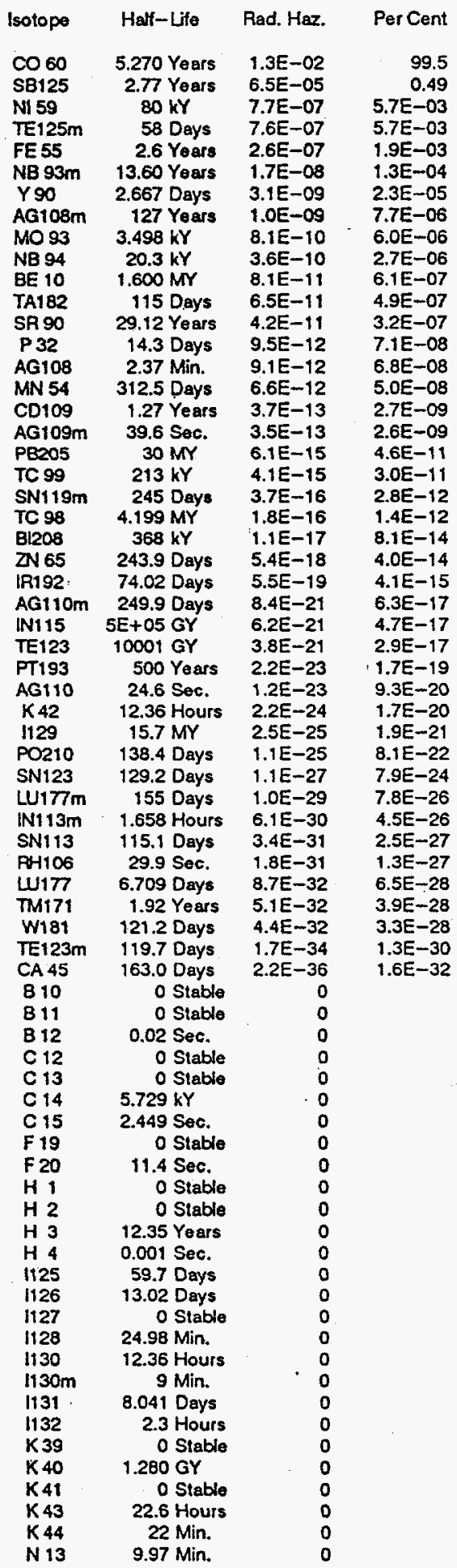


Table 2.11. (contd)

Table 11-E Decay Time $=40$ Years

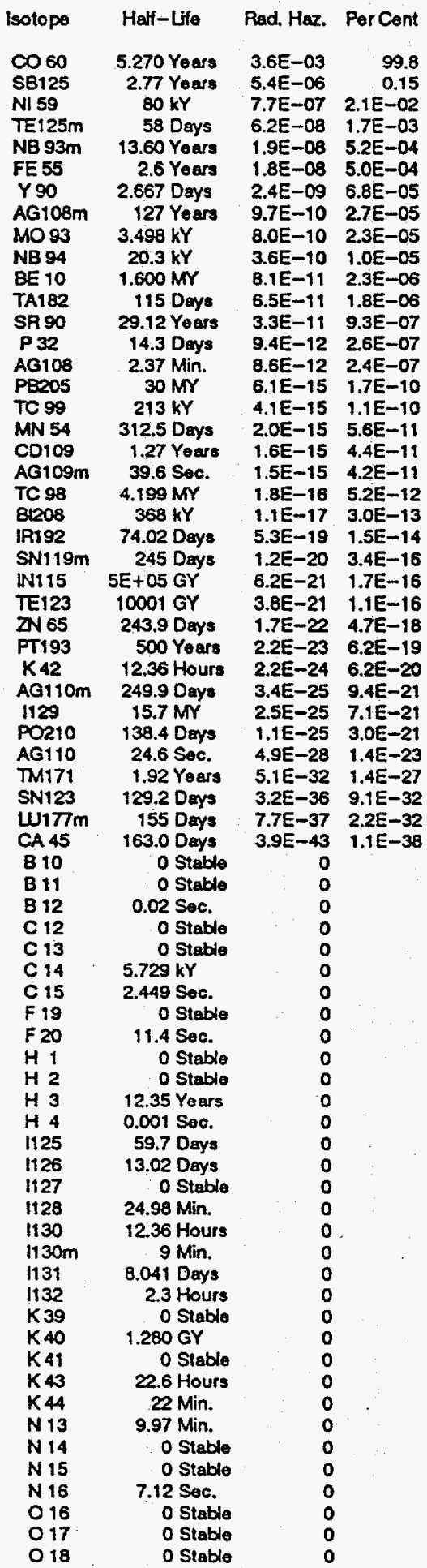

Table 11-F. Decay Time $=100$ Years

$\begin{array}{lcrr}\text { Isotope } & \text { Halt-Life } & \text { Rad. Haz. } & \text { Per Cent } \\ \text { CO } 60 & 5.270 \text { Years } & 1.3 E-06 & \\ \text { NI } 59 & 80 \text { kY } & 7.7 E-07 & 36.8 \\ \text { NB 93m } & 13.60 \text { Years } & 2.1 E-08 & 1.00 \\ \text { MO 93 } & 3.498 \text { kY } & 7.9 E-10 & 3.7 E-02 \\ \text { AG108m } & 127 \text { Years } & 7.0 E-10 & 3.3 E-02 \\ \text { Y 90 } & 2.667 \text { Days } & 5.8 E-10 & 2.7 E-02 \\ \text { NB 94 } & 20.3 \text { kY } & 3.6 E-10 & 1.7 E-02 \\ \text { BE 10 } & 1.600 \text { MY } & 8.1 E-11 & 3.8 E-03 \\ \text { TA182 } & 115 \text { Days } & 6.5 E-11 & 3.1 E-03 \\ \text { P 32 } & 14.3 \text { Days } & 8.8 E-12 & 4.1 E-04 \\ \text { SR90 } & 29.12 \text { Years } & 8.0 E-12 & 3.8 E-04 \\ \text { AG108 } & 2.37 \text { Min. } & 6.2 E-12 & 2.9 E-04 \\ \text { SB125 } & 2.77 \text { Years } & 1.6 E-12 & 7.6 E-05 \\ \text { TE125m } & 58 \text { Days } & 1.9 E-14 & 8.8 E-07 \\ \text { PB205 } & 30 \text { MY } & 6.1 E-15 & 2.9 E-07 \\ \text { TC 99 } & 213 \text { kY } & 4.1 E-15 & 1.9 E-07 \\ \text { FE 55 } & 2.6 \text { Years } & 2.0 E-15 & 9.6 E-08 \\ \text { TC 98 } & 4.199 \text { MY } & 1.8 E-16 & 8.7 E-09 \\ \text { BI208 } & 368 \text { kY } & 1.1 E-17 & 5.1 E-10 \\ \text { IR192 } & 74.02 \text { Days } & 4.5 E-19 & 2.1 E-11 \\ \text { IN115 } & 5 E+05 \text { GY } & 6.2 E-21 & 2.9 E-13 \\ \text { TE123 } & 10001 \text { GY } & 3.8 E-21 & 1.8 E-13 \\ \text { P193 } & 500 \text { Years } & 2.2 E-23 & 1.0 E-15 \\ \text { K42 } & 12.36 \text { Hours } & 2.2 E-24 & 1.0 E-16 \\ \text { I129 } & 15.7 \text { MY } & 2.5 E-25 & 1.2 E-17 \\ \text { PO210 } & 138.4 \text { Days } & 1.1 E-25 & 5.1 E-18 \\ \text { CD109 } & 1.27 \text { Years } & 9.5 E-30 & 4.5 E-22 \\ \text { AG109m } & 39.6 \text { Sec. } & 9.0 E-30 & 4.2 E-22 \\ \text { B 10 } & 0 \text { Stabl } & & \end{array}$

B

\section{B}

811
$B 12$
$C 12$
$C 13$

C 13

C 15

F 19

F 20

H 1

H 2

$\begin{array}{rl}H & 3 \\ H & 4\end{array}$

H 4

1126
1127

1128

1130

$1130 \mathrm{~m}$

1131

1132

K39
$K 40$

K 41

K 43

K44

N 13

N 14

N 15

N 16

016

017

018

019
$P 31$

P 33

P 34

S 32

S 33

S 34

535

0 Stable

O Stabla

0.02 Sec.

o Stable

O Stable

$5.729 \mathrm{kY}$

2.449 Sec.

0 Stable

11.4 Soc.

0 Stable

o Stable

12.35 Years

0.001 Sec.

59.7 Days

13.02 Days

o Stable

24.98 Min.

12.36 Hours

9 Min.

8.041 Days

2.3 Hours

$1.280 \mathrm{GY}$

- Stable

22.6 Hours

22 Min.

9.97 Min.

0 Stable

0 Stable
12 Sec.

- Stable

O Stable

0 Stable

29 Sec.

0 Stable

25 Days

12.4 Sec.

O Stable

- Stable

88 Days

o Stable 
Table 2.11. (contd)

Table $11-G$. Decey Time $=300$ Years

\begin{tabular}{|c|c|c|c|}
\hline isotope & Half-Lite & Rad. Haz. & Per Cent \\
\hline Ni 59 & $80 \mathrm{kr}$ & 7.6E-07 & 97.1 \\
\hline NB 93m & & 2.1E-08 & $\begin{array}{r}2.72 \\
\end{array}$ \\
\hline MO93 & $\begin{array}{r}3,498 \mathrm{kY} \\
20.3 \mathrm{kY}\end{array}$ & $\begin{array}{l}7.6 E-10 \\
3.6 E-10\end{array}$ & $\begin{array}{l}9.7 E-02 \\
4.5 E-02\end{array}$ \\
\hline AGto8m & 127 Years & $2.4 E-10$ & $3.0 E-02$ \\
\hline BE 10 & $1.600 \mathrm{MY}$ & $8.1 E-11$ & $1.0 E-02$ \\
\hline TA182 & 115 Deys & $6.5 E-11$ & $8.3 E-03$ \\
\hline$P_{32}$ & 14.3 Days & $7.1 E-12$ & $9.0 E-04$ \\
\hline$Y 90$ & 2.667 Days & $4.9 E-12$ & $6.3 E-04$ \\
\hline AG108 & $2.37 \mathrm{Min}$. & $2.1 E-12$ & $2.7 E-04$ \\
\hline SA90 & 29,12 Years & $6.8 E-14$ & $8.7 E-06$ \\
\hline PB205 & $30 \mathrm{Mr}$ & $6.1 E-15$ & 7.7E-07 \\
\hline TC 99 & $213 \mathrm{kY}$ & $4.1 E-15$ & 5.2E-07 \\
\hline TC 98 & $4.199 \mathrm{MY}$ & $1.8 E-16$ & $2.3 E-08$ \\
\hline $\mathrm{B} 1208$ & $368 \mathrm{kY}$ & $1.1 E-17$ & $1.4 E-09$ \\
\hline$\infty 060$ & 5.270 Years & $5.0 E-18$ & $6.4 E-10$ \\
\hline IR192 & 74.02 Days & $2.5 E-19$ & $3.1 E-11$ \\
\hline IN115 & $5 \mathrm{E}+05 \mathrm{GY}$ & $6.2 E-21$ & $7.9 E-13$ \\
\hline TE123 & $10001 \mathrm{GY}$ & $3.8 E-21$ & $4.8 E-13$ \\
\hline PT193 & 500 Years & $1.6 E-23$ & $2.0 E-15$ \\
\hline$K 42$ & 12.36 Hours & $2.2 E-24$ & $2.8 E-16$ \\
\hline 1129 & $15.7 \mathrm{MY}$ & $2.5 E-25$ & $3.2 E-17$ \\
\hline PO210 & 138.4 Deys & $1.1 E-25$ & $1.4 E-17$ \\
\hline B 10 & 0 Stable & 0 & \\
\hline B11 & 0 Stable & 0 & \\
\hline B 12 & 0.02 Soc. & 0 & \\
\hline C 12 & O Stablo & 0 & \\
\hline C 13 & O Stable & 0 & \\
\hline C 14 & $5.729 \mathrm{kY}$ & 0 & \\
\hline C 15 & 2.449 Soc. & 0 & \\
\hline F 19 & O Stable & 0 & \\
\hline $\mathrm{F} 20$ & 11.4 Sec. & 0 & \\
\hline $\begin{array}{ll}H_{1} & 1 \\
H & 2\end{array}$ & $\begin{array}{l}\text { O Stable } \\
\text { O Stable }\end{array}$ & 0 & \\
\hline $\begin{array}{l}\mathrm{H}_{2} \\
\mathrm{H} \\
3\end{array}$ & $\begin{array}{l}0 \text { Stable } \\
\text { 12.35 Years }\end{array}$ & $\begin{array}{l}0 \\
0\end{array}$ & \\
\hline $\begin{array}{ll}H_{3} & 3 \\
H & 4\end{array}$ & $\begin{array}{l}12.35 \text { Years } \\
0.001 \text { Sec. }\end{array}$ & $\begin{array}{l}0 \\
0\end{array}$ & \\
\hline $\begin{array}{l}H 4 \\
1125\end{array}$ & $\begin{array}{l}0.001 \text { Sec. } \\
59.7 \text { Deys }\end{array}$ & $\begin{array}{l}0 \\
0\end{array}$ & \\
\hline $\begin{array}{l}1125 \\
1126\end{array}$ & & $\begin{array}{l}0 \\
0\end{array}$ & \\
\hline $\begin{array}{l}1126 \\
1127\end{array}$ & $\begin{array}{c}\text { 13.02 Days } \\
\text { O Stable }\end{array}$ & $\begin{array}{l}0 \\
0\end{array}$ & \\
\hline $\begin{array}{l}1127 \\
1128\end{array}$ & $\begin{array}{l}\text { O Stable } \\
\text { 24.98 Min. }\end{array}$ & $\begin{array}{l}0 \\
0\end{array}$ & \\
\hline $\begin{array}{l}1128 \\
1130\end{array}$ & & $\begin{array}{l}0 \\
0\end{array}$ & \\
\hline $\begin{array}{l}1130 \\
1130 \mathrm{~m}\end{array}$ & $\begin{array}{c}\text { 12.36 Hours } \\
9 \mathrm{Min} .\end{array}$ & $\begin{array}{l}0 \\
0\end{array}$ & \\
\hline $\begin{array}{l}1130 \mathrm{~m} \\
1131\end{array}$ & $\begin{array}{c}9 \mathrm{Min} . \\
8.041 \text { Days }\end{array}$ & $\begin{array}{l}0 \\
0\end{array}$ & \\
\hline 1132 & 2.3 Hours & 0 & \\
\hline$K 39$ & o Stable & 0 & \\
\hline K 40 & $1.280 \mathrm{Gr}$ & 0 & \\
\hline K 41 & O Stable & 0 & \\
\hline K 43 & 22.6 Hours & 0 & \\
\hline K 44 & $22 \mathrm{Min}$. & 0 & \\
\hline$N 13$ & $9.97 \mathrm{Min}$. & 0 & \\
\hline N 14 & O Stable & 0 & \\
\hline$N 15$ & o Stable & 0 & \\
\hline$N 16$ & 7.12 Sec. & 0 & \\
\hline O 16 & O Stable & 0 & \\
\hline 017 & o Stable & 0 & \\
\hline 018 & O Stable & 0 & \\
\hline 019 & 29 Sec. & 0 & \\
\hline P 31 & o Stabla & 0 & \\
\hline P 33 & 25 Days & 0 & \\
\hline P 34 & $12.4 \mathrm{Sec}$ & 0 & \\
\hline S 32 & O Stable & 0 & \\
\hline$\$ 33$ & 0 Stable & 0 & \\
\hline S 34 & 0 Stable & 0 & \\
\hline S 35 & 88 Days & 0 & \\
\hline S 36 & 0 Stable & 0 & \\
\hline S 37 & 5.06 Min. & 0 & \\
\hline$\vee 49$ & 330.0 Days & 0 & \\
\hline$\vee 50$ & $4 E+07 \mathrm{GY}$ & 0 & \\
\hline$\vee 51$ & O Stable & 0 & \\
\hline$\vee 52$ & $3.75 \mathrm{Min}$. & 0 & \\
\hline
\end{tabular}

Table $11-H$. Decay Time $=1000$ Years

\begin{tabular}{|c|c|c|c|}
\hline Isotope & Helf-Life & Rad. Haz. & Per Cent \\
\hline NI 59 & $80 \mathrm{kY}$ & $7.6 \mathrm{E}-07$ & 97.1 \\
\hline NB 93m & 13.60 Yoars & $2.1 E-08$ & 2.74 \\
\hline MO 93 & $3.498 \mathrm{kY}$ & $6.6 E-10$ & 8.5E-02 \\
\hline NB 94 & $20.3 \mathrm{kY}$ & $3.5 E-10$ & $4.5 \bar{E}-02$ \\
\hline BE 10 & $1.600 \mathrm{Mr}$ & $8.1 E-11$ & $1.0 E-02$ \\
\hline TA182 & 115 Days & $6.5 E-11$ & $8.4 E-03$ \\
\hline AG108m & 127 Years & $5.2 E-12$ & $6.6 E-04$ \\
\hline$P_{32}$ & 14.3 Days & $3.4 E-12$ & $4.3 E-04$ \\
\hline AG108 & $2.37 \mathrm{Min}$. & $4.6 E-14$ & $5.9 E-06$ \\
\hline PB205 & $30 \mathrm{MY}$ & $6.1 E-15$ & $7.8 E-07$ \\
\hline TC 99 & $213 \mathrm{kY}$ & $4.0 E-15$ & 5.2E-07 \\
\hline TC 98 & $4.199 \mathrm{Mr}$ & $1.8 \mathrm{E}-16$ & $2.4 E-08$ \\
\hline$B 1208$ & $368 \mathrm{kY}$ & $1.1 E-17$ & $1.4 E-09$ \\
\hline Y90 & 2.667 Days & $2.9 E-19$ & $3.7 E-11$ \\
\hline IR192 & 74.02 Days & $3.8 \mathrm{E}-20$ & $4.8 \mathrm{E}-12$ \\
\hline IN115 & $5 E+05 \mathrm{GY}$ & $6.2 E-21$ & $8.0 E-13$ \\
\hline SA 90 & 29.12 Years & $4.0 E-21$ & $5.1 E-13$ \\
\hline TE123 & $10001 \mathrm{GY}$ & $3.8 E-21$ & $4.9 E-13$ \\
\hline PT193 & 500 Years & $8.0 E-24$ & $1.0 E-15$ \\
\hline 1129 & $15.7 \mathrm{Mr}$ & $2.5 E-25$ & $3.2 E-17$ \\
\hline PO2to & 138.4 Days & $1.1 E-25$ & $1.4 E-17$ \\
\hline$K 42$ & 12.36 Hours & $9.1 E-31$ & $1.2 E-22$ \\
\hline B 10 & O Stable & 0 & \\
\hline 811 & o Stable & 0 & \\
\hline 812 & 0.02 Sec. & 0 & \\
\hline C 12 & O Stable & 0 & \\
\hline C 13 & o Stable & 0 & \\
\hline C 14 & $5.729 \mathrm{kY}$ & 0 & \\
\hline C 15 & 2.449 Sec. & 0 & \\
\hline F 19 & O Stable & 0 & \\
\hline$F 20$ & 11.4 Sec. & 0 & \\
\hline HI & O Stable & 0 & \\
\hline H2 & O Stable & 0 & \\
\hline H 3 & 12.35 Years & 0 & \\
\hline H4 & 0.001 Sec. & 0 & \\
\hline 1125 & 59.7 Days & 0 & \\
\hline 1126 & 13.02 Days & 0 & \\
\hline 1127 & 0 Stable & 0 & \\
\hline 1128 & 24.98 Min. & 0 & \\
\hline 1130 & 12.36 Hours & 0 & \\
\hline $1130 \mathrm{~m}$ & 9 Min. & 0 & \\
\hline 1131 & 8.041 Days & 0 & \\
\hline 1132 & 2.3 Hours & 0 & \\
\hline $\mathrm{K} 39$ & O Stable & 0 & \\
\hline$K 40$ & $1.280 \mathrm{GY}$ & 0 & \\
\hline K41 & O Stable & 0 & \\
\hline$K 43$ & 22.6 Hours & 0 & \\
\hline$K 44$ & $22 \mathrm{Min}$. & 0 & \\
\hline N 13 & 9.97 Min. & 0 & \\
\hline N 14 & O Stable & 0 & \\
\hline N 15 & 0 Stable & 0 & \\
\hline$N 16$ & 7.12 Sec. & 0 & \\
\hline 016 & 0 Stable & 0 & \\
\hline 017 & O Stable & 0 & \\
\hline 018 & o Stablo & 0 & \\
\hline 019 & 29 Sec. & 0 & \\
\hline$P_{31}$ & O Stable & 0 & \\
\hline P 33 & 25 Days & 0 & \\
\hline P 34 & 12.4 Sec. & 0 & \\
\hline S 32 & O Stable & 0 & \\
\hline S 33 & 0 Stable & 0 & \\
\hline S 34 & O Stable & 0 & \\
\hline \$ 35 & 88 Days & 0 & \\
\hline S 36 & 0 Stable & 0 & \\
\hline s 37 & $5.06 \mathrm{Min}$. & 0 & \\
\hline V 49 & 330.0 Days & 0 & \\
\hline V 50 & $4 \mathrm{E}+07 \mathrm{GY}$ & 0 & \\
\hline V 51 & O Stable & 0 & \\
\hline V 52 & 3.75 Min. & 0 & \\
\hline V 53 & $1.61 \mathrm{Min}$. & 0 & \\
\hline
\end{tabular}


Table 2.11. (contd)

Table 11-1. Decay Time $=3000$ Years

\begin{tabular}{|c|c|c|c|}
\hline lsotopo & Half-Life & Rad. Haz. & Per Cont \\
\hline N1 59 & $80 \mathrm{kY}$ & $7.5 E-07$ & 97.1 \\
\hline NB 93m & 13.60 Yoars & $2.1 E-08$ & 2.78 \\
\hline MO 93 & $3.498 \mathrm{kY}$ & 4.5E-10 & $5.8 E-02$ \\
\hline NB 94 & $20.3 \mathrm{kY}$ & 3.3E-10 & 4.2E-02 \\
\hline BE 10 & $1.600 \mathrm{Mr}$ & $8.1 E-11$ & $1.1 E-02$ \\
\hline TAt82 & 115 Days & $6.5 E-11$ & $8.5 E-03$ \\
\hline P 32 & 14.3 Days & $4.0 E-13$ & 5.2E-05 \\
\hline PB205 & $30 \mathrm{Mr}$ & $6.1 E-15$ & $7.9 E-07$ \\
\hline TC 99 & $213 \mathrm{kV}$ & $4.0 E-15$ & $5.2 E-07$ \\
\hline $\begin{array}{l}\text { TC } 98 \\
\text { AG108m }\end{array}$ & $\begin{array}{l}4.199 \mathrm{Mr} \\
127 \mathrm{Y}_{\mathrm{BOF}}\end{array}$ & $\begin{array}{l}1.8 E-16 \\
9.4 E-17\end{array}$ & 2.4E-08 \\
\hline 81208 & $\begin{array}{l}127 \text { Years } \\
368 \mathrm{kY}\end{array}$ & $\begin{array}{l}9.4 E-17 \\
1.1 E-17\end{array}$ & $\begin{array}{l}1.2 \mathrm{E}-08 \\
1.4 \mathrm{E}-09\end{array}$ \\
\hline AG108 & 2.37 Min. & 8.3E-19 & $1.1 E-10$ \\
\hline N115 & $5 E+05$ GY & $6.2 E-21$ & $8.1 E-13$ \\
\hline IR192 & 74.02 Days & $5.4 E-21$ & $7.0 E-13$ \\
\hline TE123 & $10001 \mathrm{GY}$ & $3.8 \mathrm{E}-21$ & $4.9 E-13$ \\
\hline PT193 & 500 Yoars & $2.0 E-24$ & 2.6E-16 \\
\hline 1129 & $15.7 \mathrm{MY}$ & $2.5 E-25$ & 3.3E-17 \\
\hline PO210 & 138.4 Days & $1.1 E-25$ & $1.4 E-17$ \\
\hline SR 90 & 29.12 Years & $6.0 E-42$ & 7.7E-34 \\
\hline $\begin{array}{l}\text { B 10 } \\
\text { B 11 }\end{array}$ & $\begin{array}{l}\text { O Stable } \\
\text { o Stablo }\end{array}$ & & \\
\hline B 12 & 0.02 Soc. & 0 & \\
\hline$C_{12}$ & 0 Stable & 0 & \\
\hline$C_{13}$ & O Stable & 0 & \\
\hline C 14 & $5.729 \mathrm{KY}$ & 0 & \\
\hline $\begin{array}{l}C_{15} 15 \\
F_{19}\end{array}$ & $\begin{array}{l}2.449 \text { Sec. } \\
0 \text { Stablo }\end{array}$ & $\begin{array}{l}0 \\
0\end{array}$ & \\
\hline$F 20$ & 11.4 Soc. & 0 & \\
\hline $\mathrm{HI}$ & o Stable & 0 & \\
\hline $\begin{array}{ll}H 2 \\
42\end{array}$ & 0 Stable & 0 & \\
\hline H 3 & 12.35 Yoars & 0 & \\
\hline $\begin{array}{l}\text { H 4 } \\
1125\end{array}$ & $\begin{array}{l}0.001 \text { Sec. } \\
59.7 \text { Days }\end{array}$ & $\begin{array}{l}0 \\
0\end{array}$ & \\
\hline 1126 & $\begin{array}{l}59.7 \text { Days } \\
\text { 13.02 Deys }\end{array}$ & $\begin{array}{l}0 \\
0\end{array}$ & \\
\hline 1127 & 0 Stable & 0 & \\
\hline 1728 & 24.98 Min. & 0 & \\
\hline 1130 & 12.36 Hours & 0 & \\
\hline $1130 \mathrm{~m}$ & $9 \mathrm{Min}$. & 0 & \\
\hline 1131 & 8.041 Days & 0 & \\
\hline 1132 & 2.3 Hours & 0 & \\
\hline K39 & O Stablo & 0 & \\
\hline$K 40$ & $1.280 \mathrm{GY}$ & 0 & \\
\hline K41 & O Stablo & 0 & \\
\hline K 42 & 12.36 Hours & 0 & \\
\hline$K 43$ & 22.6 Hours & 0 & \\
\hline K 44 & $22 \mathrm{Min}$. & 0 & \\
\hline N 13 & $9 . \overline{97} \mathrm{Min}$. & 0 & \\
\hline N 14 & 0 Stable & 0 & \\
\hline N 15 & 0 Stablo & 0 & \\
\hline N 16 & 7.12 Soc. & 0 & \\
\hline 016 & O Stable & 0 & \\
\hline 017 & 0 Stablo & 0 & \\
\hline 018 & 0 Stablo & 0 & \\
\hline 019 & 29 Soc. & 0 & \\
\hline P 31 & O Stable & 0 & \\
\hline P 33 & 25 Days & 0 & \\
\hline P 34 & 12.4 Soc. & 0 & \\
\hline S 32 & O Stable & 0 & \\
\hline S 33 & 0 Stable & 0 & \\
\hline 534 & o Stable & 0 & \\
\hline S 35 & 88 Days & 0 & \\
\hline S 36 & 0 Stable & 0 & \\
\hline S37 & $5.06 \mathrm{Min}$ & 0 & \\
\hline V 49 & 330.0 Days & 0 & \\
\hline V 50 & $4 E+07 G Y$ & 0 & \\
\hline V 51 & o Stable & 0 & \\
\hline V 52 & 3.75 Min. & 0 & \\
\hline V 53 & 1.61 Min. & 0 & \\
\hline V 54 & $55 \mathrm{Sec}$. & 0 & \\
\hline
\end{tabular}


Table 2.12. Relative Radiation Hazard of Surface-Deposited Actinides from Hanford Reactor Fuel for Various Decay Times

Table 12-A. Docey Time = 1 Yoar

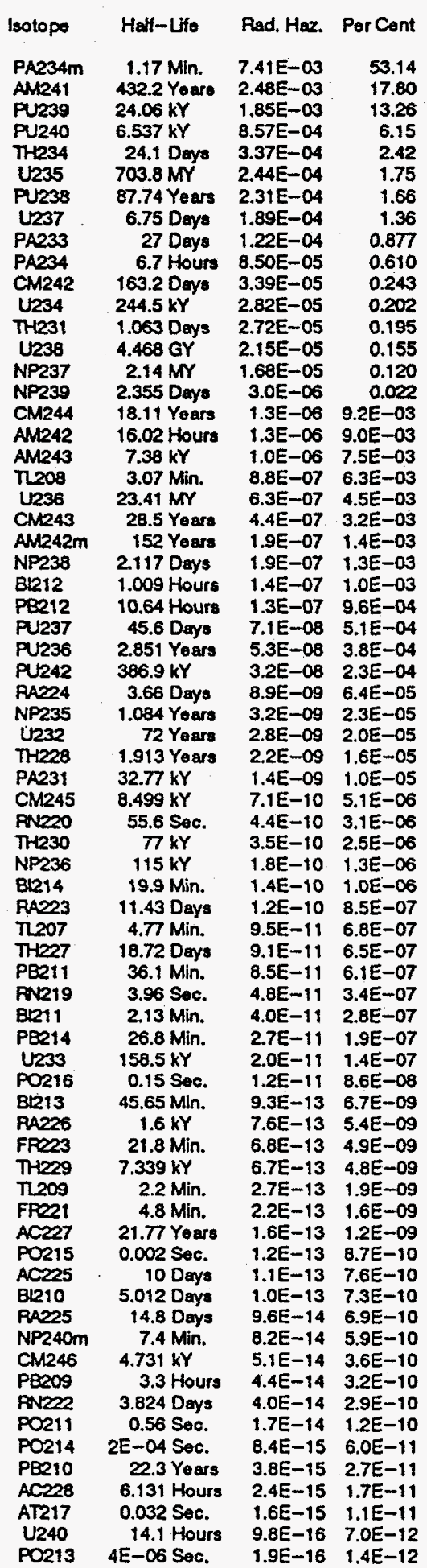

Toble 12-B. Docoy Time $=3$ Years

\begin{tabular}{|c|c|c|c|}
\hline & aif-Lito & Haz. & Per Ce \\
\hline A234m & 17 & $\begin{array}{l}3 \\
3\end{array}$ & \\
\hline 12. & 10 & -03 & 0.12 \\
\hline J240 & & & 4.69 \\
\hline & & & \\
\hline & & & i. \\
\hline 12 & is & & 1.25 \\
\hline & & & 0.943 \\
\hline & & & 0.670 \\
\hline & 6 & & \\
\hline & & -05 & .155 \\
\hline+12 & & & 0.149 \\
\hline & & & 0.118 \\
\hline & & -05 & 0.092 \\
\hline & & & 0.017 \\
\hline & & & 0.012 \\
\hline & & & $8.6 E-03$ \\
\hline & & $-\infty 6$ & $E-03$ \\
\hline & & -06 & $E-03$ \\
\hline & & & \\
\hline$y$ & & $\begin{array}{l}-07 \\
-07\end{array}$ & \\
\hline Bl21 & & & \\
\hline & & -07 & \\
\hline & & & -03 \\
\hline & & & \\
\hline & & -08 & \\
\hline & & -08 & \\
\hline & & -08 & \\
\hline & & -09 & -05 \\
\hline & & & \\
\hline & & -09. & \\
\hline & & -09 & \\
\hline & & & \\
\hline & & & \\
\hline & & & \\
\hline & & & \\
\hline$C M$ & & & \\
\hline & & & \\
\hline & & & \\
\hline & & & \\
\hline & & & \\
\hline & & & \\
\hline & & & \\
\hline & & & \\
\hline & & & \\
\hline & & & \\
\hline & & & \\
\hline & & & \\
\hline & & & \\
\hline & & & \\
\hline & & & \\
\hline & & & \\
\hline & & & \\
\hline & & & \\
\hline & & & \\
\hline & & & \\
\hline & & & \\
\hline & & -13 & \\
\hline & & & \\
\hline & & -13 & \\
\hline & & & \\
\hline & $7.4 \mathrm{Mi}$ & 14 & 4.5 \\
\hline & & & \\
\hline & & & \\
\hline & & 5. & \\
\hline & 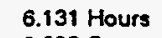 & $1.7 \mathrm{E}-14$ & \\
\hline & & 15 & \\
\hline & & & \\
\hline & & & \\
\hline
\end{tabular}


Table 2.12. (contd)

Table 12-C. Decay Time $=10$ Years

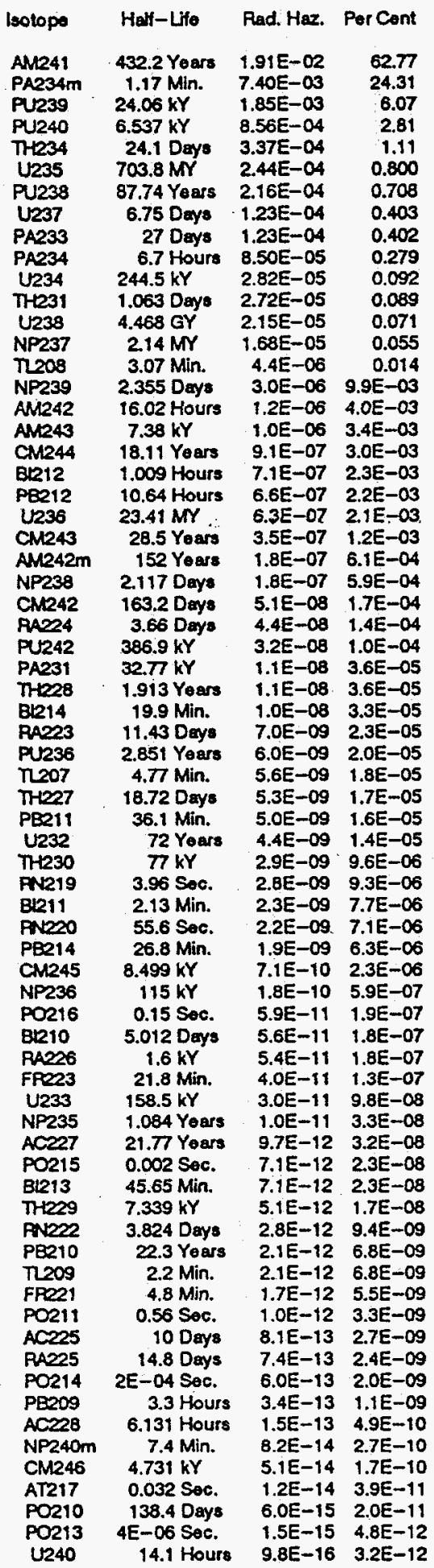

Table 12-D. Decay Time $=30$ Years

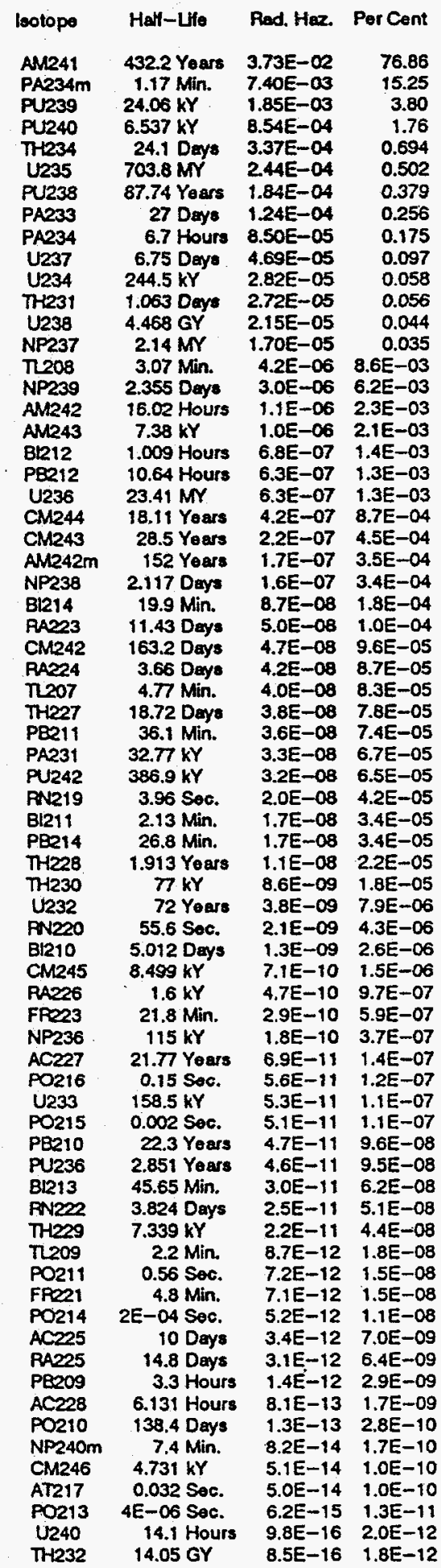


Table 2.12. (contd)

Table 12-E Decay Time $\approx 40$ Yoars



Table 12-F. Docay Time $=100$ Years

\begin{tabular}{|c|c|c|c|}
\hline lsotopo & Hali-Lito & Rad. Haz. & Per Cent \\
\hline AM241 & 432.2 Years & $4.39 E-02$ & 79.80 \\
\hline PA234m & 1.17 $\mathrm{Min}$. & $7.40 E-03$ & 13.47 \\
\hline PU239 & $24.06 \mathrm{kY}$ & $1.84 E-03$ & 3.35 \\
\hline PU240 & $6.537 \mathrm{kY}$ & $8.48 E-04$ & 1.54 \\
\hline$T H+234$ & 24.1 Days & $3.37 E-04$ & 0.613 \\
\hline U235 & $703.8 \mathrm{MY}$ & $2.44 E-04$ & 0.443 \\
\hline PA233 & 27 Deys & 1.32E-04 & 0.240 \\
\hline PU238 & 87.74 Yoars & $1.06 E-04$ & 0.193 \\
\hline PA234 & 6.7 Hours & $8.50 E-05$ & 0.155 \\
\hline U234 & $244.5 \mathrm{kY}$ & 2.82E-05 & 0.051 \\
\hline$T+231$ & 1.063 Days & $2.72 E-05$ & 0.050 \\
\hline U238 & 4.468 GY & $2.15 E-05$ & 0.039 \\
\hline NP237 & $2.14 \mathrm{MY}$ & $1.81 E-05$ & 0.033 \\
\hline NP239 & 2.355 Days & $3.0 \mathrm{E}-06$ & $5.5 E-03$ \\
\hline T208 & 3.07 Min. & 2.1E-06 & $3.9 E-03$ \\
\hline U237 & 6.75 Deys & $1.6 E-06$ & $2.9 E-03$ \\
\hline AM243 & $7.38 \mathrm{kY}$ & $1.0 E-06$ & $1.9 E-03$ \\
\hline B1214 & 19.9 Min. & $9.5 E-07$ & $1.7 \mathrm{E}-03$ \\
\hline AM242 & 16.02 Hours & $8.0 E-07$ & $1.5 E-03$ \\
\hline U236 & $23.41 \mathrm{Mr}$ & $6.3 \mathrm{E}-07$ & $1.2 E-03$ \\
\hline 81212 & 1.009 Hours & $3.5 \mathrm{E}-07$ & $6.3 E-04$ \\
\hline PE212 & 10.64 Hours & 3.2E -07 & $5.9 E-04$ \\
\hline PA223 & 11.43 Days & 3.2E-07 & $5.8 \mathrm{E}-04$ \\
\hline 7207 & 4.77 Min. & $2.6 E-07$ & $4.7 E-04$ \\
\hline$T H+227$ & 18.72 Days & $2.5 E-07$ & $4.5 \mathrm{E}-04$ \\
\hline PEe11 & 36.1 Min. & 2.3E-07 & 4.2E-04 \\
\hline PE214 & 26.8 Min. & $1.8 \mathrm{E}-07$. & 3.3E- 04 \\
\hline FN219 & $3.96 \mathrm{Sec}$. & 1.3E-07 & $2.4 \mathrm{E}-04$ \\
\hline AM $242 \pi$ & 152 Years & $1.2 E-07$ & 2.2E -04 \\
\hline NP238 & 2.117 Days & $1.2 E-07$ & 2.2E-04 \\
\hline PA231 & $32.77 \mathrm{kr}$ & 1.1E-07 & $2.0 E-04$ \\
\hline Bl211 & 2.13 Min. & $1.1 E-07$ & 2.0E-04 \\
\hline CM243 & 28.5 Years & $4.0 E-08$ & $7.2 E-05$ \\
\hline CM242 & 163.2 Days' & $3.4 E-08$ & $6.2 E-05$ \\
\hline PU242 & $386.9 \mathrm{kY}$ & $3.2 E-08$ & $5.8 E-05$ \\
\hline B1210 & 5.012 Daya & $3.0 E-08$ & 5.5E-05 \\
\hline CM244 & 18.11 Yoars & $2.9 E-08$ & 5.3E-05 \\
\hline TH230 & $77 \mathrm{kY}$ & $2.9 E-08$ & $5.2 E-05$ \\
\hline RA224 & 3.66 Days & $2.2 E-08$ & $3.9 E-05$ \\
\hline TH2228 & 1.913 Yoars & 5.4E-09 & $9.9 \mathrm{E}-06$ \\
\hline PA226 & $1.6 \mathrm{kY}$ & $5.1 E-09$ & $9.3 E-06$ \\
\hline U232 & 72 Years & $2.0 E-09$ & $3.6 E-06$ \\
\hline FRE23 & 21.8 Min. & $1.8 \mathrm{E}-09$ & $3.4 E-06$ \\
\hline PE210 & 22.3 Yoars & $1.1 E-09$ & $2.0 \mathrm{E}-06$ \\
\hline BNEZO & 55.6 Soc. & $1.1 E-09$ & $1.9 \mathrm{E}-06$ \\
\hline CM245 & $8.499 \mathrm{kY}$ & $7.1 E-10$ & $1.3 E-06$ \\
\hline AC227 & 21.77 Years & $4.5 E-10$ & $8.2 E-07$ \\
\hline PO215 & 0.002 Soc. & 3.3E-10 & $6.0 E-07$ \\
\hline RN2222 & 3.824 Days & $2.7 E-10$ & $4.9 E-07$ \\
\hline B1213 & 45.65 Min. & $2.1 E-10$ & $3.8 E-07$ \\
\hline NP236 & $115 \mathrm{kY}$ & $1.8 \mathrm{E}-10$ & 3.3E-07 \\
\hline TH2229 & $7.339 \mathrm{kY}$ & $1.5 \mathrm{E}-10$ & $2.8 E-07$ \\
\hline U233 & $158.5 \mathrm{kY}$ & $1.4 E-10$ & $2.5 E-07$ \\
\hline$\pi 209$ & 2.2 Min. & $6.1 E-11$ & $1.1 E-07$ \\
\hline PO214 & 2E-04 Sec. & $5.7 E-11$ & $1.0 \mathrm{E}-07$ \\
\hline FF221 & 4.8 Min. & $5.0 E-11$ & $9.0 E-08$ \\
\hline PO211 & 0.56 & $4.6 \mathrm{E}-11$ & $8.4 E-08$ \\
\hline PO216 & 0.15 Sec. & $2.9 E-11$ & $5.2 E-08$ \\
\hline$A C 225$ & 10 Days & $2.4 E-11$ & $4.4 E-08$ \\
\hline RA225 & 14.8 Days & $2.2 E-11$ & $4.0 E-08$ \\
\hline PB209 & 3.3 Hours & $1.0 E-11$ & $1.8 \mathrm{E}-08$ \\
\hline AC228 & 6.131 Hours & $3.9 E-12$ & $7.1 E-09$ \\
\hline PO210 & 138.4 Days & $3.2 E-12$ & $5.9 \mathrm{E}-09$ \\
\hline AT217 & 0.032 Sec & $3.5 E-13$ & $6.4 E-10$ \\
\hline PU236 & 2.851 Years & $1.0 E-13$ & $1.9 E-10$ \\
\hline NP240m & 7.4 Min. & $8.2 E-14$ & $1.5 E-10$ \\
\hline CM246 & $4.731 \mathrm{kV}$ & $5.0 E-14$ & $9.1 E-11$ \\
\hline PO213 & $4 E-06$ Sec. & $4.4 \mathrm{E}-14$ & $7.9 E-11$ \\
\hline TH232 & $14.05 \mathrm{GY}$ & $2.8 E-15$ & $5.2 E-12$ \\
\hline U240 & 14.1 Hours & $9.8 E-16$ & $1.8 \mathrm{E}-12$ \\
\hline
\end{tabular}


Table 2.12. (contd)

Tablo 12-Q. Decay Time $=300$ Years

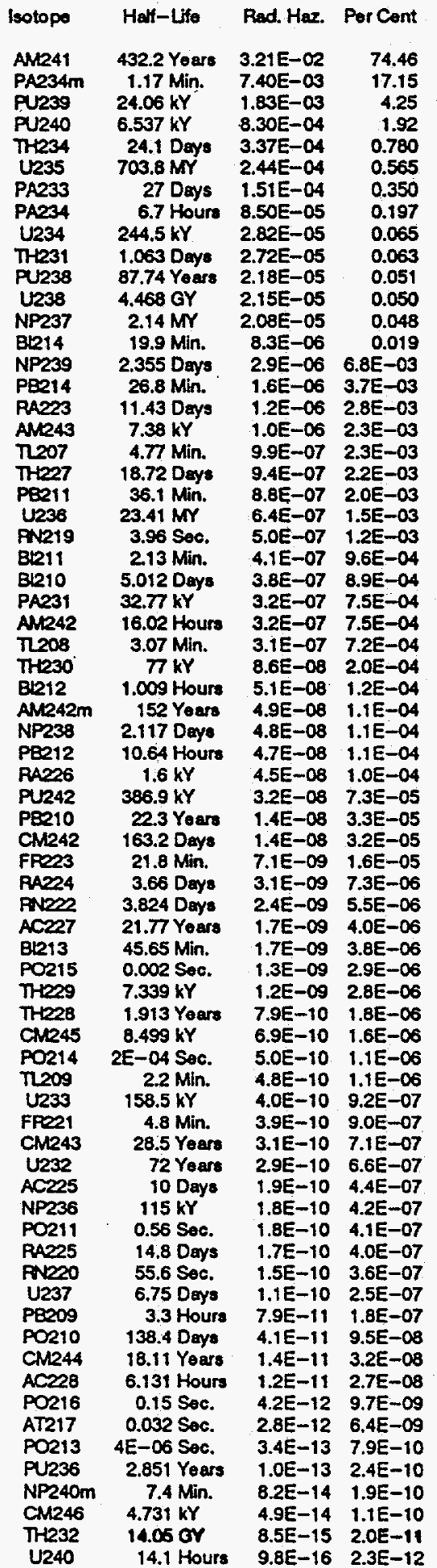

Table $12-H$. Decay Tine $=1000$ Years

\begin{tabular}{|c|c|c|c|}
\hline lootope & Hâf-Uite & Rad. Hez. & Por Cont \\
\hline 241 & 432.2 Years & $1.05 E-02$ & 48.62 \\
\hline PA234m & $1.17 \mathrm{Min}$. & $7.40 E-03$ & 34.42 \\
\hline PU239 & $24.06 \mathrm{kY}$ & $1.80 E-03$ & 8.35 \\
\hline PU240 & $6.537 \mathrm{kY}$ & $7.71 E-04$ & 3.58 \\
\hline TH234 & 24.1 Days & 3.37E-04 & 1.57 \\
\hline U235 & $703.8 \mathrm{Mr}$ & $2.44 \mathrm{E}-04$ & 1.14 \\
\hline PA233 & 27 Days & $1.86 E-04$ & 0.863 \\
\hline PA234 & 6.7 Hours & 8.50E-05 & 0.395 \\
\hline B1214 & $19.9 \mathrm{Min}$. & 8.34E-05 & 0.388 \\
\hline U234 & $244.5 \mathrm{kY}$ & $2.82 E-05$ & 0.131 \\
\hline$T H 231$ & 1.063 Days & $2.73 E-05$ & 0.127 \\
\hline NP237 & $2.14 \mathrm{MV}$ & $2.55 E-05$ & 0.119 \\
\hline U238 & $4.468 \mathrm{GY}$ & $2.15 E-05$ & 0.100 \\
\hline PB214 & 26.8 Min. & $1.59 \mathrm{E}-05$ & 0.074 \\
\hline B1210 & 5.012 Days & $4.8 E-06$ & 0.022 \\
\hline RA223 & 11.43 Days & $4.5 E-06$ & 0.021 \\
\hline TL207 & 4.77 Min. & $3.7 E-06$ & 0.017 \\
\hline TH2227 & 18.72 Days & $3.5 E-06$ & 0.016 \\
\hline PB211 & 36.1 Min. & $3.3 E-06$ & 0.015 \\
\hline NP239 & 2.355 Days & $2.8 E-06$ & 0.013 \\
\hline PN219 & 3.96 Soc. & $1.8 E-06$ & $8.6 E-03$ \\
\hline B1211 & 2.13 Min. & $1.5 E-06$ & $7.1 E-03$ \\
\hline PA231 & $32.77 \mathrm{kY}$ & $1.1 E-06$ & $5.0 E-03$ \\
\hline AM243 & $7.38 \mathrm{kY}$ & $9.5 E-07$ & $4.4 E-0.3$ \\
\hline U236 & $23.41 \mathrm{MY}$ & $6.5 E-07$ & $3.0 \mathrm{E}-03$ \\
\hline RAD26 & $1.6 \mathrm{kY}$ & $4.5 E-07$ & $2.1 E-03$ \\
\hline TH1230 & $77 \mathrm{KY}$ & $2.8 E-07$ & $1.3 E-03$ \\
\hline PB210 & 22.3 Years & $1.8 E-07$ & 8.2E-04 \\
\hline PU238 & 87.74 Yoars & $8.8 E-08$ & $\begin{array}{l}4.1 E-04 \\
15 E-04\end{array}$ \\
\hline $\begin{array}{l}\text { PU242 } \\
\text { FP223 }\end{array}$ & $\begin{array}{l}386.9 \mathrm{kY} \\
21 . \mathrm{Min}\end{array}$ & $\begin{array}{l}3.2 E-08 \\
2.6 E-08\end{array}$ & $\begin{array}{l}1.5 E-04 \\
1.2 E-04\end{array}$ \\
\hline $\begin{array}{l}\text { FP223 } \\
\text { PN2222 }\end{array}$ & $\begin{array}{l}\text { 21.8 Min. } \\
\text { 3.824 Days. }\end{array}$ & $2.4 E-08$ & $1.1 E-04$ \\
\hline B1213 & $45.65 \mathrm{Min}$. & $1.9 E-08$ & $8.9 E-05$ \\
\hline $7 \mathrm{H} 229$ & $7.339 \mathrm{kY}$ & $1.4 E-08$ & $6.4 E-05$ \\
\hline AM242 & 16.02 Hours & $1.3 E-08$ & $6.1 E-05$ \\
\hline AC2227 & 21.77 Yoars & $6.3 E-09$ & $2.9 \mathrm{E}-05$ \\
\hline$\pi 209$ & $2.2 \mathrm{Min}$. & $5.6 \mathrm{E}-09$ & $2.6 \mathrm{E}-05$ \\
\hline PO214 & 2E-04 Soc. & $5.0 E-09$ & 2.3E-05 \\
\hline PO215 & 0.002 Soc. & 4.7E-09 & 2.2E-05 \\
\hline FRe21 & $4.8 \mathrm{Min}$. & $4.5 E-09$ & 2.1E-05 \\
\hline AC225 & 10 Days & $2.2 E-09$ & $1.0 \mathrm{E}-05$ \\
\hline AM242m & 52 Yoars & $2.0 E-09$ & $9.4 E-06$ \\
\hline PA225 & 14.8 Days & $2.0 \mathrm{E}-09$ & $9.2 E-06$ \\
\hline NP238 & 2.117 Days & $2.0 E-09$ & $9.1 E-06$ \\
\hline U233 & $158.5 \mathrm{kY}$ & $1.5 E-09$ & $7.0 \mathrm{E}-06$ \\
\hline PB209 & 3.3 Hours & $9.1 E-10$ & $4.2 E-06$ \\
\hline PO211 & $0.56 \mathrm{~S}$ & $6.6 E-10$ & $3.0 E-06$ \\
\hline CM245 & $8.499 \mathrm{kY}$ & $6.6 E-10$ & $3.0 E-06$ \\
\hline CM242 & 163.2 Days & $5.6 \mathrm{E}-10$ & $2.6 \mathrm{E}-06$ \\
\hline TL208 & $3.07 \mathrm{Min}$. & $5.3 E-10$ & 2.5E-06 \\
\hline PO210 & 138.4 Days & $5.1 \mathrm{E}-10$ & $2.4 E-06$ \\
\hline NP236 & $115 \mathrm{kY}$ & $1.8 E-10$ & 8.3E-07 \\
\hline 81212 & 1.009 Hours & 8.6E-11 & 4.0E-07 \\
\hline PB212 & 10.64 Hours & $8.0 E-11$ & 3.7E-07 \\
\hline AC228 & 131 Hours & $3.9 E-11$ & $1.8 E-07$ \\
\hline AT217 & 0.032 Sec. & $3.2 E-11$ & 1.5E-07 \\
\hline PA:224 & 3.66 Days & $5.3 E-12$ & $2.5 E-08$ \\
\hline PO213 & 4E-06 Soc. & $4.0 E-12$ & $1.8 E-08$ \\
\hline TH+228 & 1.913 Years & $1.3 E-12$ & $6.3 E-09$ \\
\hline U232 & 72 Years & $4.5 E-13$ & $2.1 E-09$ \\
\hline RN220 & 55.6 Sec. & $2.6 E-13$ & $1.2 E-09$ \\
\hline Pu236 & 2.851 Year: & $1.0 E-13$ & $4.7 E-10$ \\
\hline NP240m & $7.4 \mathrm{Min}$. & $8.2 E-14$ & $3.8 E-10$ \\
\hline CM246 & $4.731 \mathrm{kY}$ & $4.4 E-14$ & $2.0 E-10$ \\
\hline U237 & 6.75 Days & $3.0 E-14$ & $1.4 E-10$ \\
\hline TH1232 & $14.05 \mathrm{GY}$ & $2.9 E-14$ & $1.3 E-10$ \\
\hline PO216 & $0.15 \mathrm{Sec}$ & $7.1 E-15$ & $3.3 E-11$ \\
\hline U240 & 14.1 Hours & $9.8 E-16$ & $4.5 E-12$ \\
\hline Pues & $82.61 \mathrm{MY}$ & $1.4 E-16$ & $6.6 E-13$ \\
\hline CMPA & 28.5 Years & $1.2 E-17$ & 5.7E \\
\hline
\end{tabular}


Table 2.12. (contd)

Table 12-1. Decay Time $=3000$ Yeare

\begin{tabular}{|c|c|c|c|}
\hline & H-Lifo & Haz. & r Cont \\
\hline & $17 \mathrm{~N}$ & & 52.15 \\
\hline & & & 23 \\
\hline & & & 23 \\
\hline & & & \\
\hline & & & 3. \\
\hline & 24.1 & & .83 \\
\hline & & & .07 \\
\hline & & & .69 \\
\hline & & & 0.230 \\
\hline & & & .713 \\
\hline 23 & & & \\
\hline 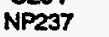 & & & \\
\hline & & & 31 \\
\hline 238 & & & 181 \\
\hline$x$ & & & \\
\hline 122 & & & 186 \\
\hline & & & \\
\hline 21 & & & $\begin{array}{l}0.046 \\
0.038\end{array}$ \\
\hline & & & 0.026 \\
\hline & & & 0.026 \\
\hline & & & 0.019 \\
\hline & & & \\
\hline ID? & & & \\
\hline & & & \\
\hline & & & -03 \\
\hline & & & \\
\hline & & & \\
\hline & & & \\
\hline & & & \\
\hline$m$ & & & 04 \\
\hline 2021 & & & \\
\hline 14 & & & \\
\hline & & & \\
\hline & & & \\
\hline & & & \\
\hline & 21. & & \\
\hline 15 & & & \\
\hline 09 & & & \\
\hline $3 ?$ & & & \\
\hline & & & \\
\hline & & & -05 \\
\hline & & & \\
\hline 208 & & & \\
\hline & & & \\
\hline & & & \\
\hline & & & \\
\hline & & & \\
\hline $\begin{array}{l}\mathrm{PO} 13 \\
\mathrm{PAO2}_{4}\end{array}$ & $4 E_{3}$ & & \\
\hline & & & \\
\hline & & & \\
\hline & & & -09 \\
\hline & & & \\
\hline & & & \\
\hline & & & \\
\hline & & & \\
\hline & & & -10 \\
\hline & 14. & & \\
\hline & & & \\
\hline & & 6. & \\
\hline & & & \\
\hline & & & \\
\hline & & 3.4 & $2.9 \mathrm{E}-11$ \\
\hline & & & \\
\hline & & & \\
\hline & & & \\
\hline
\end{tabular}




\subsection{Contributions from Fallout Radionuclides}

A major source of radionuclide contamination of the earth's surface, including the Hanford Site, is fallout from nuclear weapons testing. Fallout levels were particularly high at mid-latitudes and were also relatively high at our particular longitude. Based on studies conducted by the DOE Health and Safety Laboratory $\left(\right.$ HASL $^{(7)}$ ), currently called the Environmental Measurements Safety Laboratory (EML), we estimate that the cumulative Sr-90 fallout from nuclear weapons testing on the Hanford Site reached about $70 \mathrm{mCi} / \mathrm{Km}^{2}\left(1.6 \times 10^{5}\right.$ disintegrations per minute per square meter) in January 1967. Above-ground nuclear testing continued involving megaton devices through 1980 . While a precise estimate of the current surface concentration of the resulting Sr-90 on the Hanford Site and that of other long-lived radionuclides will require a considerable effort and is currently underway, it is estimated that the current $\mathrm{Sr}-90$ concentration is approximately $50 \mathrm{mCi} / \mathrm{Km}^{2}\left(1.1 \times 10^{5} \mathrm{dpm} / \mathrm{m}^{2}\right)$.

Tables 3.1, 13-A, B, and C, and Tables 3.2, 14-A, B, and C show the quantities of each of the long-lived fission products and actinide radionuclides from a 10 kiloton detonation after decay periods of 10,30 , and 40 years as calculated by the ORIGEN2 Code. An estimate of the concentrations of the various long-lived radionuclides in addition to $\mathrm{Sr}-90$ on the Hanford Site can be made by calculating the ratio of each radionuclide of interest to that of Sr-90 from Table 13B or 14B and multiplying this ratio by the above concentration of $\mathrm{Sr}-90\left(1.1 \times 10^{5} \mathrm{dpm} / \mathrm{m}^{2}\right)$. A more precise estimate of the fallout source for each radionuclide is currently being made and will be published separately.

One important fact from the above estimate is that fallout from nuclear weapons testing has been substantial and may very well be responsible for the majority of most of the current fission and actinide radionuclides in surface soils on the Hanford Site. A second important fact is that since the ratio of Pu-240 to Pu-239 is 2 to 3 times higher in nuclear weapons fallout than in the weapons plutonium produced at Hanford, measurements of the plutonium isotopic ratio in Hanford soils will allow its origin to be determined-fallout versus Hanford production. 
Table 3.1. Radionuclide Content of Fission Products from a 10 KT Burst at Various Decay Times

Table 13-A. Decay Time = 10 Years

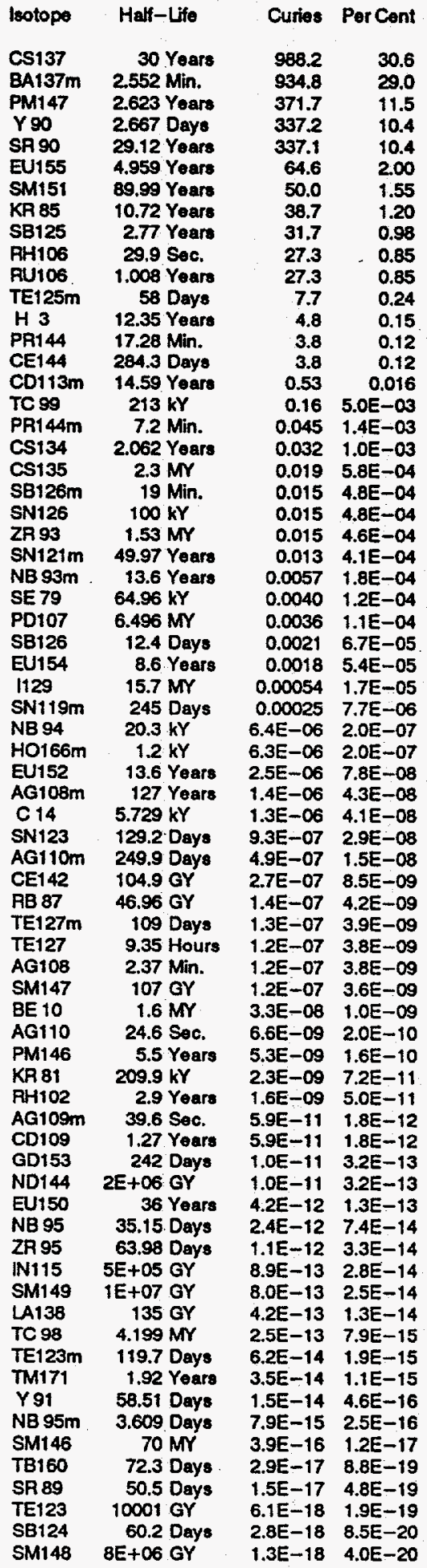

Table 13-B. Decey Time $=30$ Years

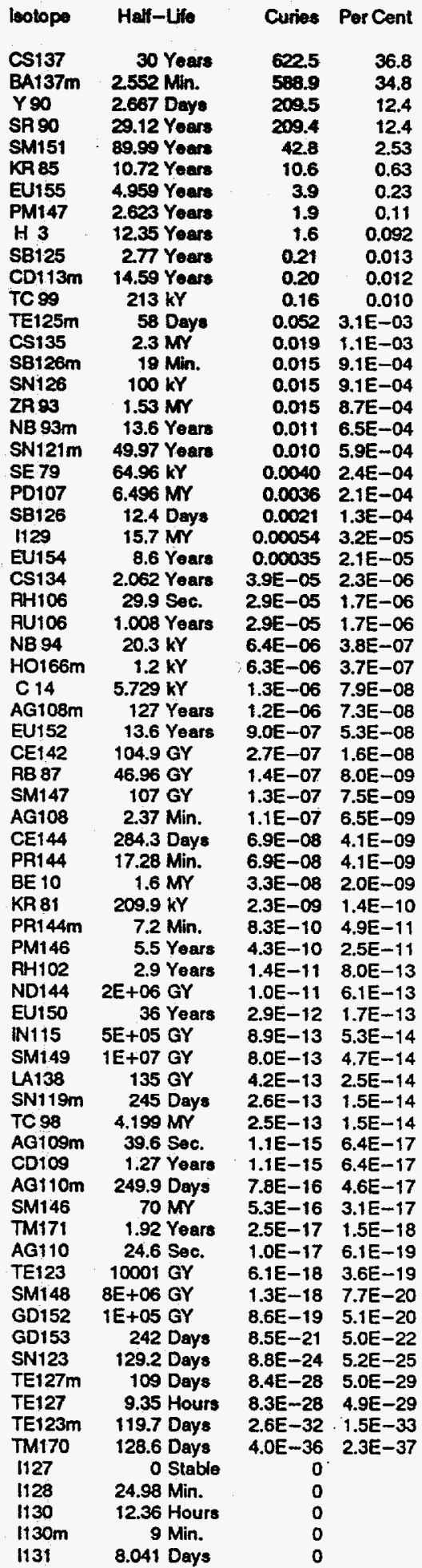


Table 3.1. (contd)

Table 13-C. Decay Time $=40$ Years

\begin{tabular}{|c|c|c|c|}
\hline stope & Hall-Lite & Curies & Per Cent \\
\hline$\$ 137$ & 30 & 494.1 & \\
\hline 90 & 2.667 & $\begin{array}{l}467.4 \\
165.1\end{array}$ & $\begin{array}{l}34.9 \\
12.3\end{array}$ \\
\hline & & 165.0 & 12.3 \\
\hline 31 & & 39.7 & 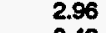 \\
\hline 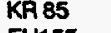 & 1 & 5.6 & 0.42 \\
\hline . & 4 & 0.98 & 0.073 \\
\hline & 12. & 0.89 & 0.066 \\
\hline & 2 & 0.16 & 0.012 \\
\hline 7 & 2.6 & 0.13 & 0.010 \\
\hline & sars & 0.13 & $9.4 E-03$ \\
\hline & 2.3 & 0.019 & $1.4 E-03$ \\
\hline 0 & 2.77 Yoars & 0.017 & $1.3 E-03$ \\
\hline $6 m$ & $19 !$ & 0.015 & $1.1 E-03$ \\
\hline & & 0.015 & $1.1 E-03$ \\
\hline & 1.5 & 0.015 & -03 \\
\hline & are & 0.012 & $E-04$ \\
\hline & 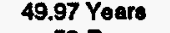 & $0 . c$ & -04 \\
\hline & & 0.0 & -04 \\
\hline & 64.5 & 0.0040 & $3.0 E-04$ \\
\hline & 6.4 & 0. & $2.7 E-04$ \\
\hline & ays & 0.0021 & $1.6 E-04$ \\
\hline & 15.7 & 0.00 & $4.0 \mathrm{E}-05$ \\
\hline 154 & are & 0.00016 & $1.2 E-05$ \\
\hline & 20. & $6.4 E-06$ & 4.8E-07 \\
\hline $56 r$ & 1. & $6.2 E-06$ & $\begin{array}{l}4.7 E-07 \\
1.0 E-07\end{array}$ \\
\hline 4 & ars & -06 & $\begin{array}{l}1.0 E-07 \\
1.0 E-07\end{array}$ \\
\hline 14 & 5.72 & 1.5 & $\begin{array}{l}-07 \\
-08\end{array}$ \\
\hline $8 m$ & pars & $\begin{array}{l}1.2 \\
5.4\end{array}$ & $\begin{array}{l}-08 \\
-08\end{array}$ \\
\hline $\begin{array}{l}152 \\
142\end{array}$ & & $\begin{array}{l}5.4 \\
2.7\end{array}$ & $\begin{array}{l}4.1 E-08 \\
2.0 E-08\end{array}$ \\
\hline 87 & $46.96 \mathrm{GY}$ & $1.4 E-07$ & $1.0 \mathrm{E}-\infty 8$ \\
\hline 147 & 107 & $1.3 E-07$ & $9.4 E-09$ \\
\hline & 2.37 Min. & -07 & $7.8 E-09$ \\
\hline & 1. & -08 & $2.5 E-09$ \\
\hline 06 & 29. & $3.0 E-08$ & $2.2 E-09$ \\
\hline 106 & 1.00 & $3.0 E-08$ & $2.2 \mathrm{E}-09$ \\
\hline & 209.9 & $2.3 E-09$ & $1.7 E-10$ \\
\hline 146 & ars & $1.2 E-10$ & $9.0 E-12$ \\
\hline 144 & $2 E+06$ & $1.0 E-11$ & $7.7 E-13$ \\
\hline 144 & 284. & $9.4 E-12$ & $7.0 E-13$ \\
\hline 144 & 17.28 & $9.4 E-12$ & $7.0 E-13$ \\
\hline 150 & gare & $2.4 E-12$ & $1.8 E-13$ \\
\hline 02 & & -12 & $9.3 E-14$ \\
\hline 5 & $5 \mathrm{E}+05$ & $8.9 E-13$ & $6.7 E-14$ \\
\hline 149 & $1 E+07$ & -13 & $6.0 E-14$ \\
\hline 138 & 135 & $4.2 E-13$ & $3.2 E-14$ \\
\hline 98 & 4.199 & $2.5 E-13$ & $1.9 E-14$ \\
\hline $4 \mathrm{~m}$ & 7.2 & $1.1 E-13$ & $8.4 E-15$ \\
\hline 1146 & 70 & $5.4 E-16$ & $4.0 E-17$ \\
\hline $9 m$ & & -18 & $6.4 E-19$ \\
\hline 3 & 1000 & $6.1 E-18$ & $4.6 E-19$ \\
\hline $99 m$ & & $4.6 E-18$ & $3.4 E-19$ \\
\hline & & & -19 \\
\hline & $8 E+$ & -18 & $9.8 E-20$ \\
\hline 52 & $1 E+C$ & -19 & $6.5 E-20$ \\
\hline & & -19 & $5.1 E-20$ \\
\hline Om & & -20 & 2.3E-21 \\
\hline & & & $3.1 E-23$ \\
\hline & & -25 & $1.8 E-26$ \\
\hline & 129 & & 2.0 \\
\hline & 10 & -38 & $5.1 E-39$ \\
\hline & ble & & \\
\hline & 24 & & \\
\hline & 12,36 & & \\
\hline & & & \\
\hline & 8.041 & & \\
\hline & & & \\
\hline 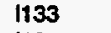 & 20.8 & 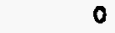 & \\
\hline & & & \\
\hline
\end{tabular}


Table 3.2. Radionuclide Content of Actinides from a $10 \mathrm{KT}$ Burst at Various Decay Times

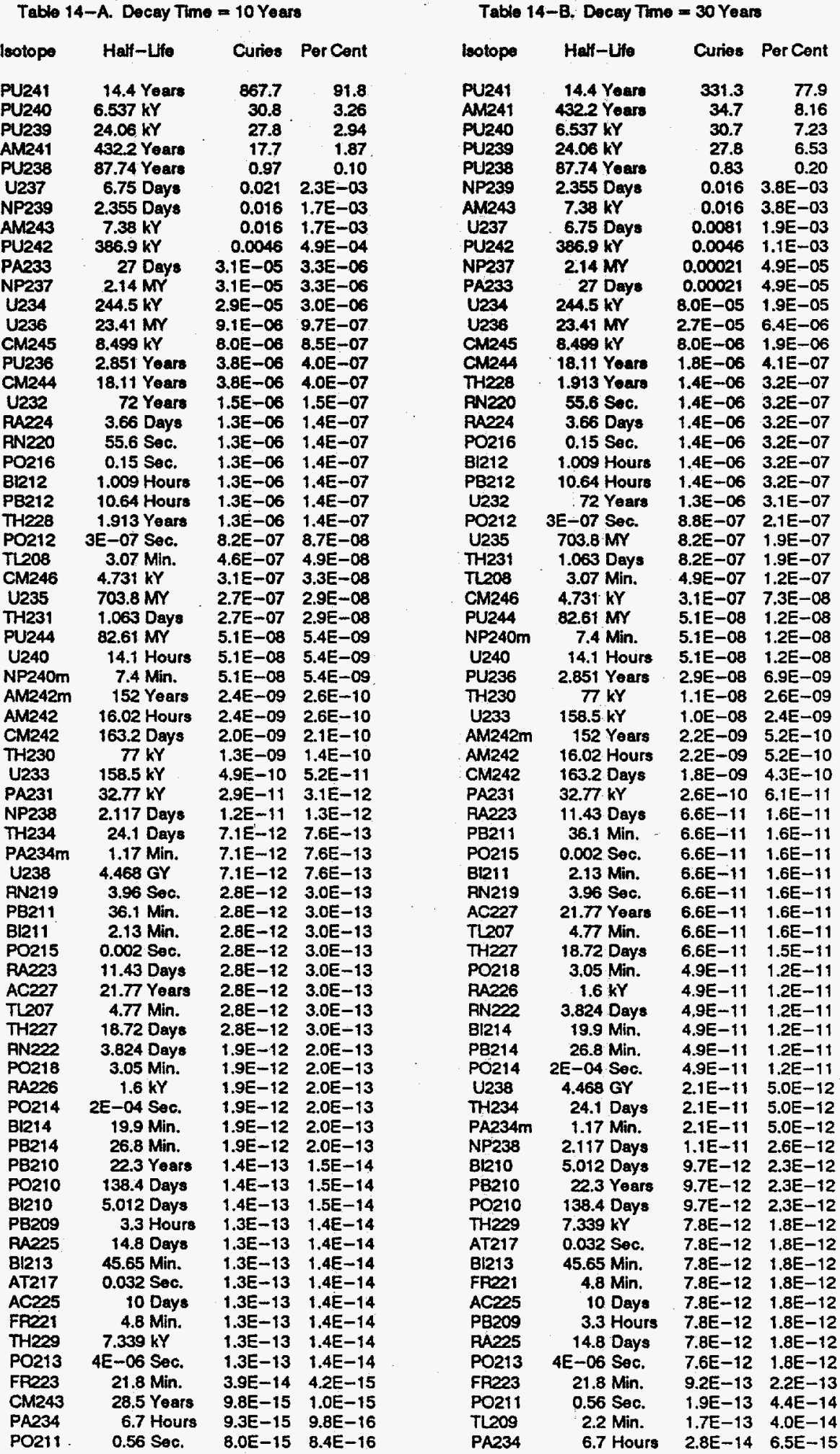


Table 3.2. (contd)

Table 14-C. Decay Time $=40$ Years

\begin{tabular}{|c|c|c|c|}
\hline 68 & Lifo & Curies & PorCon \\
\hline & & & 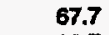 \\
\hline & & 38.3 & 12 \\
\hline & & 30.7 & 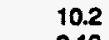 \\
\hline & & & \\
\hline & 87.74 Yea & 0.77 & $\begin{array}{r}0.25 \\
E-03\end{array}$ \\
\hline $\begin{array}{l}1243 \\
2239\end{array}$ & $\begin{array}{r}7.38 \mathrm{kY} \\
2.355 \mathrm{Dg}\end{array}$ & $\begin{array}{l}0.016 \\
0.016\end{array}$ & $\begin{array}{l}5.4 E-03 \\
5.4 E-03\end{array}$ \\
\hline & & 0.0050 & $1.7 E-03$ \\
\hline U242 & & 0.0046 & $5 E-03$ \\
\hline 237 & $14 N$ & 0.00033 & $1 E-04$ \\
\hline & $y=$ & .00033 & $1.1 E-04$ \\
\hline & $244.5 k$ & .00010 & $3.4 E-05$ \\
\hline & & SE-05 & $\begin{array}{l}1.2 E-05 \\
27 E-06\end{array}$ \\
\hline 2 & $\begin{array}{l}8.8 \\
1.0\end{array}$ & $\begin{array}{l}0 E-06 \\
2 E-06\end{array}$ & $\begin{array}{l}2.7 E-\infty 6 \\
4.1 E-07\end{array}$ \\
\hline & 10 & $1.2 E-06$ & 4.1E-07 \\
\hline PO216 & & & 4.1E-07 \\
\hline & & $\begin{array}{l}\infty 6 \\
06\end{array}$ & $\begin{array}{l}4.1 \mathrm{E}-07 \\
4.1 \mathrm{E}-07\end{array}$ \\
\hline TH228 & & & 4.1E-07 \\
\hline & 72 & $.2 E-06$ & 4.0E-07 \\
\hline 244 & 18.1 & $2 E-06$ & $\begin{array}{l}4.0 \mathrm{E}-07 \\
3.6 \mathrm{E}-07\end{array}$ \\
\hline & & & $\begin{array}{l}6 E-07 \\
6 E-07\end{array}$ \\
\hline $\begin{array}{l}1231 \\
2212\end{array}$ & & & E-07 \\
\hline & & & -0 \\
\hline M246 & & E-07 & $1.0 \mathrm{E}-0$ \\
\hline & & & \\
\hline U240 & 14 & $1 E-0 B$ & $1.7 \mathrm{E}-08$ \\
\hline NP240m & $74 \mathrm{M}$ & & 1.7E-08 \\
\hline U233 & & & $-\infty$ \\
\hline $\begin{array}{l}\text { TH230 } \\
\text { PU236 }\end{array}$ & & & $\begin{array}{l}6.4 E-09 \\
8.6 E-10\end{array}$ \\
\hline AM242m & & & \\
\hline 1242 & 16.0 & $2.1 \mathrm{E}-09$ & $\begin{array}{l}7.0 E-10 \\
5.8 E-10\end{array}$ \\
\hline 0 & & & $\begin{array}{l}5.8 E-10 \\
1.5 E-10\end{array}$ \\
\hline B & & $\begin{array}{l}10 \\
10\end{array}$ & $\begin{array}{l}1.5 \mathrm{E}-10 \\
4.9 \mathrm{E}-11\end{array}$ \\
\hline 2011 & & -10 & -11 \\
\hline 215 & & -10 & $\begin{array}{l}4.9 E-11 \\
4.9 E-11\end{array}$ \\
\hline & & & $\begin{array}{l}9 E-11 \\
9 E-11\end{array}$ \\
\hline 2227 & & $\begin{array}{r}10 \\
-10\end{array}$ & $\begin{array}{l}4.9 E-11 \\
4.9 E-11\end{array}$ \\
\hline & & & \\
\hline 227 & & -10 & $4.8 E-11$ \\
\hline 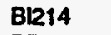 & & & $3 E-11$ \\
\hline 214 & & -10 & $8 E-11$ \\
\hline 214 & & & $E-11$ \\
\hline & & & -11 \\
\hline 222 & & & $\begin{array}{l}B E-11 \\
B E-11\end{array}$ \\
\hline & & -11 & -12 \\
\hline & & & $5 E-1$ \\
\hline & & & $5 E-1$ \\
\hline & & & $:-12$ \\
\hline & 1. & & $E-12$ \\
\hline & & & \\
\hline 25 & & -11 & $5 E-12$ \\
\hline & & & $5 E-12$ \\
\hline 13 & 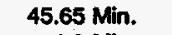 & & $.5 E-12$ \\
\hline & & & \\
\hline & & & EE-12 \\
\hline & & & $5 E-12$ \\
\hline 29 & & & $5 E-12$ \\
\hline & & 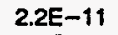 & $E-1$ \\
\hline & & $1.1 E-11$ & $3.5 E-12$ \\
\hline 223 & 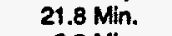 & $2.0 E-12$ & $6.7 E-13$ \\
\hline & & & \\
\hline & & $4.1 E-13$ & $1.4 E-13$ \\
\hline & $6.7 \mathrm{r}$ & $7 E-14$ & $2 E-1$ \\
\hline
\end{tabular}




\subsection{Recommendations}

The tables in this document which present the relative hazard of each radionuclide as a function of time after irradiation can be used as a guide in selecting those radionuclides which may be of concern at the time when site remediation is complete and after longer periods of time. Based on the relative amounts of each radionuclide present after years of decay and factoring in their DCG values to estimate their relative hazard, it is suggested that the radionuclides listed in Table 4.1 be included in preremediation and subsequent site surveys. The basis for this recommendation is that, in most cases, their relative abundance at 40 years after irradiation, together with their relative hazard based on their DCG values, would warrant this.

The radionuclide $\mathrm{Sm}-151$ (90 yr) has not been included in the list of radionuclides for preremediation and subsequent site surveys. Sm-151 would be very difficult and expensive to measure, and since it should follow the environmental chemistry of the rare earth Eu-154 (8.6 yr) which can be measured with comparative ease, its possible presence and amount could be estimated from measurements of Eu-154. Also, the importance of the radionuclide Cd-113m (14.59 yr) is questionable and therefore not included.

Other radionuclides which may not have a particularly high relative hazard have been added. These include I-129 and Tc-99 since they will be the principal radionuclides after very long decay periods, and they form anions which are relatively mobile in groundwater. There are several other very long-lived radionuclides which could be of concern. However, their concentrations and their potential hazard are likely to be extremely low in environmental matrices and therefore it does not seem justifiable to include them in either preremediation or subsequent surveys.

Certain natural radionuclides are included since they are easily measurable and may constitute a substantial portion of the radiation exposure. These include K-40, Ra-226, and Th-232. The radionuclides Ru-106 and Sb-125 should be included since they do form anions which are readily transported in groundwater and thus provide an indication of the transport of other radionuclides. However, their short half-lives will ensure essentially no contribution to human exposure after decay periods of 40 years or longer.

Tritium is also included in Table 4.1 since it exists as tritiated water (HTO) and is thus a precursor of all the radionuclides which are transported in subsurface water.

The selection of radionuclides in Table 4.1 which could be measured in preremediation and subsequent site surveys should only be regarded as a suggestion. The addition or deletion of radionuclides from this list could be made where specific information on concentrations and fractionation processes would warrant it.

Important information which was employed in the various calculations and considerations that went into the preparation of this document are included in Tables A.1, B.1, B.2, and B.3. Table A.1 summarizes the concentrations of major and minor elements in the fuel cladding, as well as the uranium isotopic composition of the single pass and $\mathrm{N}$ reactor fuels. Table B.1 provides a listing of the Hanford 
Table 4.1. Suggested Radionuclides to be Included in Surveys Relative to Site Remediation

\begin{tabular}{|c|c|}
\hline Radionuclide & Half-Life, Years \\
\hline $\mathrm{H}-3^{*}$ & 12.35 \\
\hline $\mathrm{K}-40$ & $1.28 \times 10^{9}$ \\
\hline Co-60 & 5.270 \\
\hline Sr-90 & 29.12 \\
\hline Tc-99 & $2.13 \times 10^{5}$ \\
\hline Ru-106 & 1.008 \\
\hline $\mathrm{Sb}-125$ & 2.77 \\
\hline I-129 & $1.57 \times 10^{7}$ \\
\hline Cs-134 & 2.062 \\
\hline Cs-137 & 30 \\
\hline $\mathrm{Eu}-152$ & 13.6 \\
\hline $\mathrm{Eu}-154$ & 8.6 \\
\hline Eu-155 & 4.96 \\
\hline $\mathrm{Ra}-226$ & 1600 \\
\hline Th-232 & $1.4 \times 10^{10}$ \\
\hline U-232 & 72 \\
\hline U-233 & $1.59 \times 10^{5}$ \\
\hline U-234 & $2.45 \times 10^{5}$ \\
\hline U-235 & $7.038 \times 10^{8}$ \\
\hline Np-237 & $2.14 \times 10^{6}$ \\
\hline Pu-238 & 87.74 \\
\hline U-238 & $4.468 \times 10^{9}$ \\
\hline Pu-239 & $2.406 \times 10^{4}$ \\
\hline $\mathrm{Pu}-240$ & 6537 \\
\hline Am-241 & 432.2 \\
\hline Pu-241 & 14.4 \\
\hline $\mathrm{Cm}-244$ & 18.11 \\
\hline
\end{tabular}

* Tritium should probably be included in ground water measurements since it is an excellent indicator and precursor of other radioactive wastes.

Default Inhalation classes which were used in determining DCG values for Hanford (see Appendix B). Table B. 2 summarizes the selected DCG values for Hanford which were used in calculating all of the relative hazards of the radionuclides after specific decay periods. Table B.3\% provides the dose conversion factors for external exposure to radionuclides assuming they were deposited on the surface of Hanford soils. 


\subsection{References}

1. Croff, A. G. 1980. ORIGEN2 - A Revised and Updated Version of the Oak Ridge Isotope Generation and Depletion Code, ORNL-5621, Oak Ridge National Laboratory, Oak Ridge, Tennessee.

2. Croff, A. G. 1980. A User's Manual for the ORIGEN2 Computer Code, ORNL/TM-7175, Oak Ridge National Laboratory, Oak Ridge, Tennessee.

3. 10 CFR 834. Radiation Protection of the Public and the Environment - Derived Concentration Guides for Air and Water; March 25, 1993. Federal Register Vol. 58, No. 56 Proposed Rules, pp. 16268-16322.

4. Napier, B. A., R. A. Peloquin, D. L. Strenge, and J. V. Ramsdell. 1988. GENII - The Hanford Environmental Radiation Dosimetry Software System. Vol. 1: Conceptual Representation; Vol. 2: Users' Manual; Vol. 3: Code Maintenance Manual, PNL-6584, Vol. 1, 2, and 3, Pacific Northwest Laboratory, Richland, Washington.

5. Kocher, D. C., and K. F. Eckerman. 1988. External Dose-Rate Conversion Factors for Calculation of Dose to the Public; DOE/EH-0070, U.S. Department of Energy, Washington, D.C., and 1989 Erratum Sheet.

6. Heeb, C. M. 1991. Uncertainties in Source Term Calculations Generated by the ORIGEN2 Computer Code for Hanford Production Reactors; PNL-7223 HEDR, Pacific Northwest Laboratory, Richland, Washington.

7. Hardy, E. P., M. W. Meyer, J. S. Allen, and L. T. Alexander. 1968. "Strontium-90 on the Earth's Surface." Nature, Vol. 219.

8. Bergsman, K. K. 1993. Hanford Irradiated Fuel Inventory Baseline, WHC-SD-CP-TI-175 Rev. 1, Westinghouse Hanford Company, Richland, Washington. 
Appendix A

\section{Basis for ORIGEN2 Calculation}




\section{Appendix A}

\section{Basis for ORIGEN2 Calculation}

ORIGEN2 calculations were made for single-pass reactor irradiations and for N-Reactor irradiations to determine radionuclide concentrations in spent fuel and cladding. Impurities in the fuel and the cladding were included in the model. The quantities are based on data of $\operatorname{Bergsman}^{(a)}$ and are given Table A.1. The brazing was also included in the model. It was assumed that the single-pass reactor fuel was all natural uranium as opposed to the actual situation where $25 \%$ of it was slightly enriched uranium. The average burnup of the single-pass reactor fuel was $728 \mathrm{MWD} / \mathrm{MTU}$. It was also assumed that all of the N-Reactor fuel was enriched to $0.947 \% \mathrm{U}-235$ when, in fact, some of it was higher enrichment. The average burnup of the $\mathrm{N}$-Reactor fuel was $1045 \mathrm{MWD} / \mathrm{MTU}$. The power density was assumed to be $10 \mathrm{MW} / \mathrm{MTU}$ for all of the fuel. For long decay times, the radionuclide concentrations are insensitive to the power density. About $90 \%$ of the fuel reprocessed at Hanford was irradiated in the single-pass reactors.

(a) Bergsman, K. K. 1993. Hanford Irradiated Fuel Inventory Baseline, WHC-SD-CP-TI-175 Rev. 1, Westinghouse Hanford Company, Richland, Washington. 
Table A.1. Composition of Hanford Reactor Fuel and Cladding

$\mathrm{N}-$ Reactor Fuel

Isotope $\quad \mathrm{g} / \mathrm{KgU}$

$U-234$

$U-235$

$\mathrm{U}-238$

0.077

9.47

990.45

$N$-Reactor Fuel Impurities

Element $\quad \mathrm{g} / \mathrm{KgU}$

Al $\quad 0.900$

$\mathrm{Be} \quad 0.010$

$\begin{array}{ll}B & 0.00025 \\ \text { Cd } & 0.00025\end{array}$

C $\quad 0.735$

Cr $\quad 0.065$

$\mathrm{Cu} \quad 0.075$

$\mathrm{H} \quad 0.002$

$\mathrm{Fe} \quad 0.400$

$\mathrm{Mg} \quad 0.025$

Mn $\quad 0.025$

$\mathrm{Ni} \quad 0.100$

N $\quad 0.075$

$\mathrm{Si} \quad 0.124$

$\mathrm{Zr} \quad 0.065$

$\mathrm{N}$-Reactor Cladding

Element $\quad \mathrm{g} / \mathrm{KgZr}$

A) $\quad 0.075$

$\mathrm{Be} \quad 0.390$

B

$\mathrm{Cd}$

$\mathrm{Cr}$

Co

$\mathrm{Cu}$

$\mathrm{Hf}$

$\mathrm{H}$

Fe

$\mathrm{Pb}$

$\mathrm{Mg}$

Mo

$\mathrm{Ni}$

$N$

Ox

$\mathrm{Si}$

$\mathrm{Na}$

Sn

$\mathrm{Ti}$

w

V

0.200

0.025

0.100

0.020

0.050

0.050

0.8

0.080

0.018

0.100

0.020

17.0

0.050

0.050

0.050

977.1
Single Pass Reactor Fuel

$\begin{array}{lr}\text { Isotope } & \mathrm{g} / \mathrm{KgU} \\ \mathrm{U}-234 & 0.055 \\ \mathrm{U}-235 & 7.11 \\ \mathrm{U}-238 & 992.84\end{array}$

Single Pass Reactor Fuel Impurities

Element $\quad \mathrm{g} / \mathrm{KgU}$

C $\quad 0.750$

$\mathrm{Cr} \quad 0.065$

$\mathrm{Fe} \quad 0.150$

$\mathrm{Mg} \quad 0.025$

$\mathrm{Mn} \quad 0.025$

$\mathrm{Ni} \quad 0.100$

$\mathrm{N} \quad 0.100$

$\mathrm{Si} \quad 0.075$
Single Pass Reactor Cladding

Element $\quad \mathrm{g} / \mathrm{KgAl}$

B

Cd

Co

$\mathrm{Cu}$

$\mathrm{Fe}$

$\mathrm{Ni}$

$\mathrm{Ni}$

Al
0.010

0.030

0.010

1.50

7.0

0.080

13.0

18.1

960.3 
Appendix B

Selection of the Derived Concentration Guide (DCG) Values 


\section{Appendix B}

\section{Selection of the Derived Concentration Guide (DCG) Values}

The Derived Concentration Guide (DCG) values are given in 10 CFR $834^{(a)}$ for two exposure modes: 1) inhalation of air and 2) ingestion of water. DCG values for air are given for inhalation classes, D, W, and Y which indicate retention half-times of days, weeks, and years. Some isotopes have values for two or even three classes, depending on the application. The Hanford default inhalation classes are given by Napier et al. ${ }^{\left({ }^{b}\right)}$ and are listed in Table B.1. Using the Hanford default inhalation classes, the appropriate DCG values were extracted from 10 CFR 834. The values are given in Table B.2 and were put onto the ORIGEN2 Decay Library. Table 18 contains a number of isotopes which do not appear in an ORIGEN2 calculation, thus the number of isotopes on the ORIGEN2 Decay Library with DCG values is significantly less than the number in Table 18. Shortlived isotopes do not have a DCG value; however, the DCG value of a long-lived parent includes the effect of the short-lived daughter.

(a) 10 CFR 834. Radiation Protection of the Public and the Environment-Derived Concentration Guides for Air and Water; March 25, 1993. Federal Register Vol. 58, No. 56 Proposed Rules, pp. 16268-16322.

(b) Napier, B. A., R. A. Peloquin, D. L. Strenge, and J. V. Ramsdell. 1988. GENII - The Hanford Environmental Radiation Dosimetry Software System. Vol. 1: Conceptual Representation; Vol. 2: Users' Manual; Vol. 3: Code Maintenance Manual, PNL-6584, Vol. 1, 2, and 3, Pacific Northwest Laboratory, Richland, Washington.

\section{B.1}


Table B.1. Hanford Default Inhalation Classes

\begin{tabular}{|c|c|c|c|}
\hline Element & Class* & Element & Class* \\
\hline$H$ & D & SB & w \\
\hline BE & $\bar{Y}$ & $T E$ & W \\
\hline 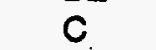 & D & 1 & D \\
\hline$N$ & D & $X E$ & $\bar{D}$ \\
\hline $\mathbf{F}$ & D & $\mathrm{cs}$ & $\bar{D}$ \\
\hline NA & D & BA & D \\
\hline SI & $w$ & LA & D \\
\hline $\mathbf{P}$ & D & CE & $Y$ \\
\hline S & $w$ & PR & $\mathbf{Y}$ \\
\hline $\mathrm{CL}$ & D & ND & $Y$ \\
\hline $\mathrm{K}$ & D & PM & $Y$ \\
\hline AR & D & SM & W \\
\hline $\mathrm{CA}$ & $w$ & EU & $w$ \\
\hline SC & $Y$ & $G D$ & $D$ \\
\hline CP & $Y$ & $\mathrm{~TB}$ & $W$ \\
\hline MN & $w$ & DY & $W$ \\
\hline FE & $w$ & $\mathrm{HO}$ & W \\
\hline $\mathrm{CO}$ & $Y$ & ER & $w$ \\
\hline NI & $w$ & TA & $Y$ \\
\hline $\mathrm{CU}$ & $D$ & $w$ & $D$ \\
\hline$Z N$ & $Y$ & RE & $W$ \\
\hline GA & $w$ & os & D \\
\hline AS & $w$ & $\mathbb{I R}$ & $Y$ \\
\hline SE & $w$ & $H G$ & D \\
\hline BA & $D$ & $\mathrm{TH}$ & $Y$ \\
\hline KR & D & RA & $w$ \\
\hline SR & $D$ & RN & D \\
\hline RB & $\mathrm{D}$ & PB & D \\
\hline$Y$ & $Y$ & $\mathrm{BI}$ & $w$ \\
\hline MO & $D$ & PO & $w$ \\
\hline ZR & $w$ & PU & $Y$ \\
\hline NB & $Y$ & $U$ & $Y$ \\
\hline TC & $w$ & TH & $Y$ \\
\hline RU & $Y$ & $A C$ & $Y$ \\
\hline PD & $Y$ & PA & $Y$ \\
\hline RH & $Y$ & FR & D \\
\hline$A G$ & $D$ & NP & $w$ \\
\hline$C D$ & D & $A M$ & $Y$ \\
\hline IN & D & $\mathrm{CM}$ & $w$ \\
\hline SN & $w$ & CF & $w$ \\
\hline
\end{tabular}


Table B.2. Derived Concentration Guide Values

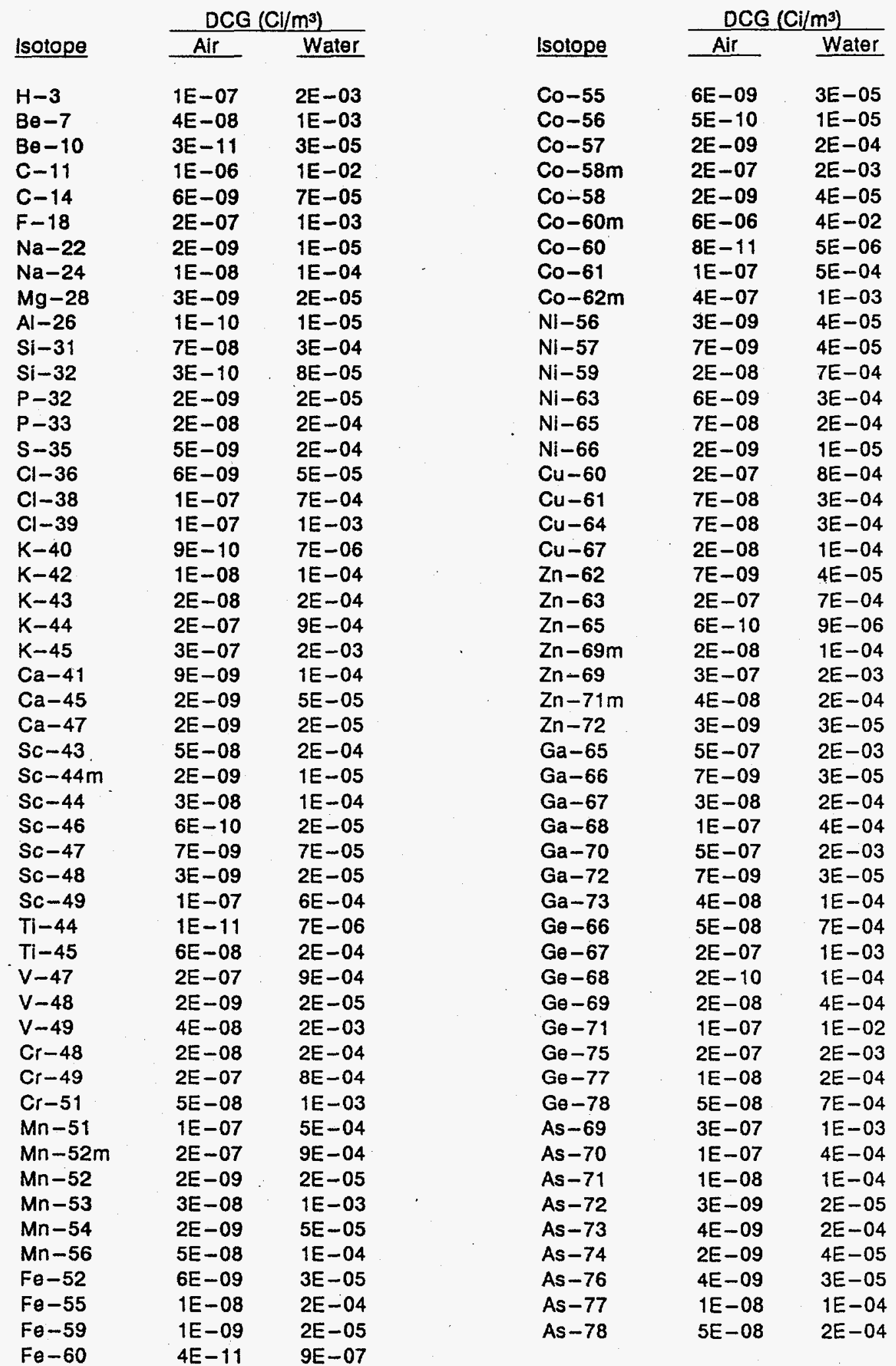

B. 3 
Table B.2. (contd)

\begin{tabular}{|c|c|c|c|c|c|}
\hline \multirow[b]{2}{*}{ Isotope } & \multicolumn{2}{|c|}{$\mathrm{DCG}\left(\mathrm{Ci} / \mathrm{m}^{3}\right)$} & \multirow[b]{2}{*}{ Isotope } & \multicolumn{2}{|c|}{ DCG $\left(C / / \mathrm{m}^{3}\right)$} \\
\hline & Air & Water & & Air & Water \\
\hline $\begin{array}{l}\text { Se }-70 \\
\text { Se }-73 \mathrm{~m}\end{array}$ & $\begin{array}{l}1 E-07 \\
3 E-07\end{array}$ & $\begin{array}{l}3 E-04 \\
9 E-04\end{array}$ & $\begin{array}{l}Z r-86 \\
Z r-88\end{array}$ & $\begin{array}{l}6 E-09 \\
1 E-09\end{array}$ & $\begin{array}{l}4 E-05 \\
1 E-04\end{array}$ \\
\hline $\mathrm{Se}-73$ & $4 E-08$ & $9 E-05$ & $2 r-89$ & $6 E-09$ & $\begin{array}{l}1 E-04 \\
4 E-05\end{array}$ \\
\hline Se -75 & $1 E-09$ & $2 E-05$ & $\mathrm{Zr}-93$ & $1 E-10$ & $9 E-05$ \\
\hline Se-79 & $1 E-09$ & $2 E-05$ & $\mathrm{Zr}-95$ & $9 E-10$ & $4 E-05$ \\
\hline Se-81m & $2 E-07$ & $7 E-04$ & $\mathrm{Zr}-97$ & $3 E-09$ & $2 E-05$ \\
\hline $\mathrm{S}_{\theta}-81$ & $6 E-07$ & $2 E-03$ & $\mathrm{Nb}-88$ & $5 E-07$ & $2 E-03$ \\
\hline Se-83 & $3 E-07$ & $9 E-04$ & $\mathrm{Nb}-89$ & $9 E-08$ & $3 E-04$ \\
\hline $8 r-74 m$ & $9 E-08$ & $6 E-04$ & $\mathrm{Nb}-89 \mathrm{~m}$ & $4 E-08$ & $1 E-04$ \\
\hline$B r-74$ & $2 E-07$ & $1 E-03$ & $\mathrm{Nb}-90$ & $6 E-09$ & $3 E-05$ \\
\hline$B r-75$ & $1 E-07$ & $1 E-03$ & $\mathrm{Nb}-93 \mathrm{~m}$ & $4 E-09$ & $5 E-03$ \\
\hline$B r-76$ & $1 E-08$ & $1 E-04$ & $\mathrm{Nb}-94$ & $4 E-11$ & $3 E-05$ \\
\hline $\mathrm{Br}-77$ & $6 E-08$ & $4 E-04$ & $\mathrm{Nb}-95 \mathrm{~m}$ & $5 E-09$ & $7 E-05$ \\
\hline $\mathrm{Br}-80 \mathrm{~m}$ & $4 E-08$ & $6 E-04$ & $\mathrm{Nb}-95$ & $3 E-09$ & $6 E-05$ \\
\hline $\mathrm{Br}-80$ & $4 E-07$ & $2 E-03$ & $\mathrm{Nb}-96$ & $6 E-09$ & $3 E-05$ \\
\hline$B r-82$ & $1 E-08$ & $8 E-05$ & $\mathrm{Nb}-97$ & $2 E-07$ & $6 E-04$ \\
\hline $\mathrm{Br}-83$ & $2 E-07$ & $2 E-03$ & $\mathrm{Nb}-98$ & $1 E-07$ & $4 E-04$ \\
\hline$B r-84$ & $1 E-07$ & $9 E-04$ & Mo-90 & $2 E-08$ & $1 E-04$ \\
\hline$R b-79$ & $3 E-07$ & $2 E-03$ & Mo-93m & $4 E-08$ & $3 E-04$ \\
\hline$R b-81 m$ & $8 E-07$ & $7 E-03$ & Mo-93 & $1 E-08$ & $1 E-04$ \\
\hline$R b-81$ & $1 E-07$ & $1 E-03$ & Mo-99 & $6 E-09$ & $5 E-05$ \\
\hline $\mathrm{Rb}-82 \mathrm{~m}$ & $4 E-08$ & $3 E-04$ & Mo-101 & $3 E-07$ & $2 E-03$ \\
\hline$R b-83$ & $2 E-09$ & $2 E-05$ & $T c-93 m$ & $7 E-07$ & $2 E-03$ \\
\hline $\mathrm{Rb}-84$ & $2 E-09$ & $1 E-05$ & Tc -93 & $2 E-07$ & $9 E-04$ \\
\hline$R b-86$ & $2 E-09$ & $1 E-05$ & $T c-94 m$ & $1 E-07$ & $5 E-04$ \\
\hline $8 b-87$ & $4 E-09$ & $3 E-05$ & $T c-94$ & $6 E-08$ & $2 E-04$ \\
\hline$R b-88$ & $2 E-07$ & $8 E-04$ & $T c-95 m$ & $5 \mathrm{E}-09$ & $1 E-04$ \\
\hline Rb-89 & $3 E-07$ & $2 E-03$ & TC-95 & $5 E-08$ & $3 E-04$ \\
\hline $\mathrm{Sr}-80$ & $3 E-08$ & $1 E-04$ & Tc-96m & $6 E-07$ & $4 E-03$ \\
\hline $\mathrm{Sr}-81$ & $2 E-07$ & $7 E-04$ & TC-96 & $5 E-09$ & $5 E-05$ \\
\hline$S r-82$ & $7 E-10$ & $7 E-06$ & $T c-97 m$ & $3 E-09$ & $1 E-04$ \\
\hline $\mathrm{Sr}-83$ & $2 E-08$ & $8 E-05$ & Tc-97 & $1 E-08$ & $9 E-04$ \\
\hline $\mathrm{sr}-85 \mathrm{~m}$ & $2 E-06$ & $6 E-03$ & Tc-98 & $7 E-10$ & $3 E-05$ \\
\hline$S_{i}-85$ & $6 E-09$ & $7 E-05$ & $T c-99 m$ & $6 E-07$ & $2 E-03$ \\
\hline $\mathrm{Sr}-87 \mathrm{~m}$ & $3 E-07$ & $1 E-03$ & Tc-99 & $2 E-09$ & $1 E-04$ \\
\hline $\mathrm{Sr}-89$ & $2 E-09$ & $2 E-05$ & $T c-101$ & $9 E-07$ & $4 E-03$ \\
\hline $\mathrm{Sr}-90$ & $5 E-11$ & $1 E-06$ & $T C-104$ & $2 E-07$ & $8 E-04$ \\
\hline$S r-91$ & $1 E-08$ & $6 E-05$ & $\mathrm{Ru}-94$ & $1 E-07$ & $4 E-04$ \\
\hline $\mathrm{St}-92$ & $2 E-08$ & $9 E-05$ & $\mathrm{Ru}-97$ & $3 E-08$ & $2 E-04$ \\
\hline$Y-86 m$ & $1 E-07$ & $6 E-04$ & $R u-103$ & $2 E-09$ & $5 E-05$ \\
\hline$Y-86$ & $7 E-09$ & $3 E-05$ & $R u-105$ & $3 E-08$ & $1 E-04$ \\
\hline$Y-87$ & $7 E-09$ & $6 E-05$ & $R u-106$ & $3 E-11$ & $6 E-06$ \\
\hline$Y-88$ & $6 E-10$ & $3 E-05$ & Rh $-99 m$ & $2 E-07$ & $5 E-04$ \\
\hline$Y-90 m$ & $3 E-08$ & $2 E-04$ & $\mathrm{Rh}-99$ & $5 E-09$ & $7 E-05$ \\
\hline$Y-90$ & $1 E-09$ & $1 E-05$ & Rh -100 & $9 E-09$ & $5 E-05$ \\
\hline$Y-91 m$ & $4 E-07$ & $4 E-03$ & $R h-101 m$ & $2 E-08$ & $2 E-04$ \\
\hline$Y-91$ & $3 E-10$ & $1 E-05$ & $R h-101$ & $4 E-10$ & $6 E-05$ \\
\hline$Y-92$ & $2 E-08$ & $7 E-05$ & $R h-102 m$ & $3 E-10$ & $4 E-05$ \\
\hline$Y-93$ & $6 E-09$ & $3 E-05$ & $A h-102$ & $1 E-10$ & $2 E-05$ \\
\hline$Y-94$ & $2 E-07$ & $8 E-04$ & An $-103 m$ & $3 E-06$ & $1 E-02$ \\
\hline$Y-95$ & $3 E-07$ & $1 E-03$ & $\mathrm{Ah}-105$ & $1 E-08$ & $1 E-04$ \\
\hline & & & $\mathrm{Rh}-106 \mathrm{~m}$ & $8 E-08$ & $2 E-04$ \\
\hline & & & $R h-107$ & $6 E-07$ & $2 E-03$ \\
\hline
\end{tabular}

B. 4 
Table B.2. (contd)

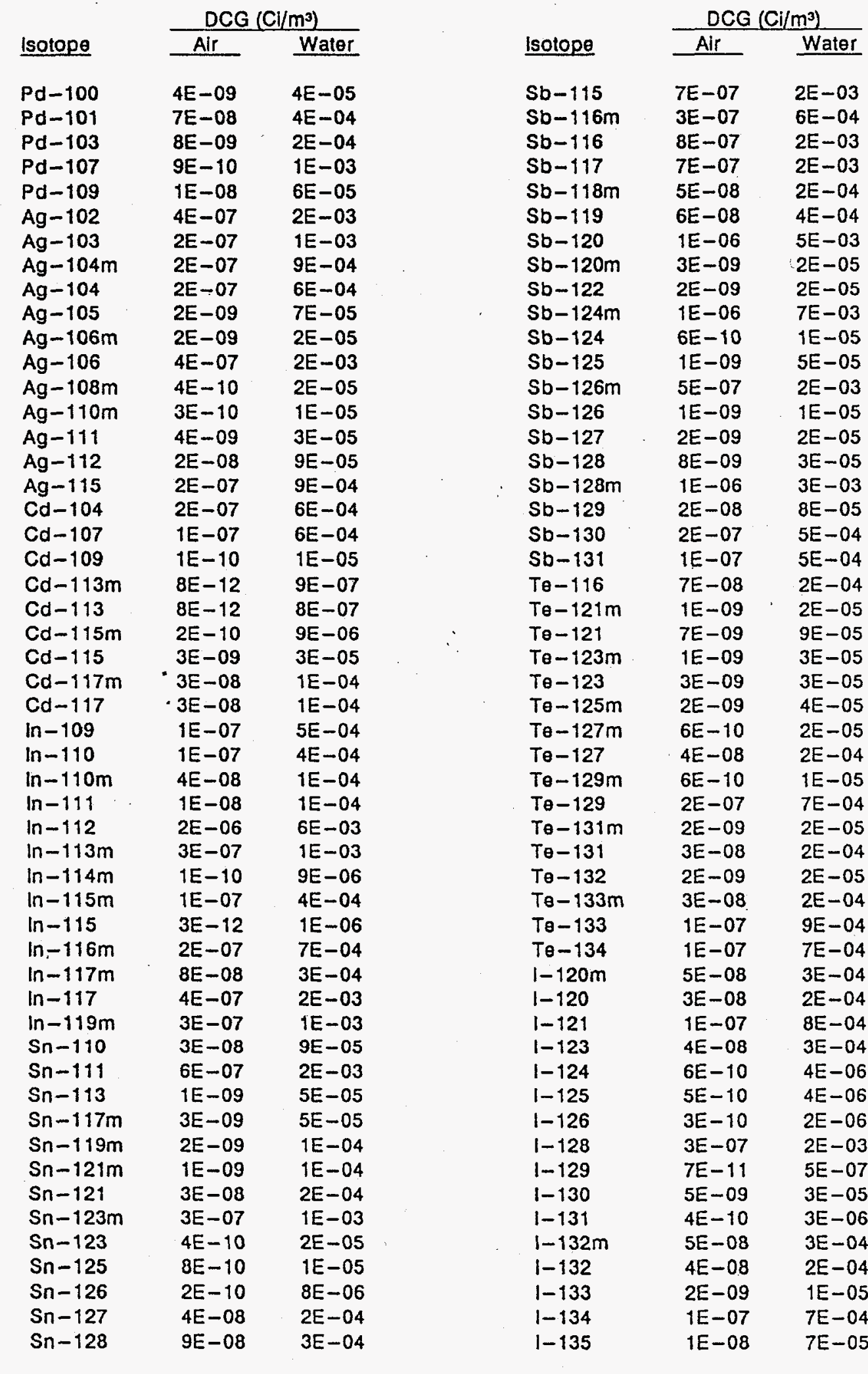


Table B.2. (contd)

\begin{tabular}{|c|c|c|c|c|c|}
\hline \multirow{2}{*}{ Isotope } & \multicolumn{2}{|c|}{$D C G\left(C i / m^{3}\right)$} & \multirow[b]{2}{*}{ Isotope } & \multicolumn{2}{|c|}{ DCG $\left(\mathrm{Ci} / \mathrm{m}^{3}\right)$} \\
\hline & Air & Water & & Air & Water \\
\hline Cs-125 & $3 E-07$ & $2 E-03$ & $\mathrm{Nd}-136$ & $1 E-07$ & $4 E-04$ \\
\hline Cs-127. & $2 E-07$ & $2 E-03$ & $N d-138$ & $1 E-08$ & $5 E-05$ \\
\hline Cs-129 & $8 E-08$ & $6 E-04$ & $\mathrm{Nd}-139 \mathrm{~m}$ & $3 E-08$ & $1 E-04$ \\
\hline Cs -130 & $4 E-07$ & $3 E-03$ & $N d-139$ & $7 E-07$ & $2 E-03$ \\
\hline Cs-131 & $7 E-08$ & $6 E-04$ & $N d-141$ & $1 E-06$ & $4 E-03$ \\
\hline Cs -132 & $9 E-09$ & $7 E-05$ & $N d-147$ & $2 E-09$ & $4 E-05$ \\
\hline Cs-134m & $3 E-07$ & $3 E-03$ & $N d-149$ & $6 E-08$ & $3 E-04$ \\
\hline$C s-134$ & $2 E-10$ & $2 E-06$ & $N d-151$ & $4 E-07$ & $2 E-03$ \\
\hline Cs-135m & $5 E-07$ & $3 E-03$ & $P m-141$ & $4 E-07$ & $2 E-03$ \\
\hline Cs-135 & $3 E-09$ & $2 E-05$ & $P m-143$ & $2 E-09$ & $1 E-04$ \\
\hline$C s-136$ & $2 E-09$ & $1 E-05$ & $P m-144$ & $3 E-10$ & $4 E-05$ \\
\hline Cs -137 & $4 E-10$ & $3 E-06$ & $P m-145$ & $4 E-10$ & $3 E-04$ \\
\hline Cs-138 & $1 E-07$ & $9 E-04$ & $P m-146$ & $1 E-10$ & $4 E-05$ \\
\hline$B a-126$ & $4 E-08$ & $2 E-04$ & $P m-147$ & $4 E-10$ & $1 E-04$ \\
\hline$B a-128$ & $4 E-09$ & $1 E-05$ & $P m-148 m$ & $7 E-10$ & $2 E-05$ \\
\hline$B a-131 m$ & $3 E-06$ & $1 E-02$ & $P m-148$ & $1 E-09$ & $1 E-05$ \\
\hline$B a-131$ & $2 E-08$ & $8 E-05$ & $P m-149$ & $4 E-09$ & $4 E-05$ \\
\hline $\mathrm{Ba}-133 \mathrm{~m}$ & $2 E-08$ & $7 E-05$ & $P m-150$ & $4 E-08$ & $1 E-04$ \\
\hline $\mathrm{Ba}-133$ & $2 E-09$ & $4 E-05$ & $P m-151$ & $7 E-09$ & $5 E-05$ \\
\hline $\mathrm{Ba}-135 \mathrm{~m}$ & $3 E-08$ & $9 E-05$ & $S m-141 m$ & $2 E-07$ & $8 E-04$ \\
\hline$B a-139$ & $7 E-08$ & $3 E-04$ & $S m-141$ & $4 E-07$ & $2 E-03$ \\
\hline$B a-140$ & $3 E-09$ & $2 E-05$ & $\mathrm{Sm}-142$ & $6 E-08$ & $2 E-04$ \\
\hline$B a-141$ & $2 E-07$ & $7 E-04$ & $S m-145$ & $1 E-09$ & $2 E-04$ \\
\hline $8 a-142$ & $3 E-07$ & $1 E-03$ & $S m-146$ & $1 E-13$ & $7 E-07$ \\
\hline La-131 & $3 E-07$ & $1 E-03$ & $S m-147$ & $2 E-13$ & $8 E-07$ \\
\hline La-132 & $2 E-08$ & $9 E-05$ & $S m-151$ & $4 E-10$ & $4 E-04$ \\
\hline La-135 & $2 E-07$ & $1 E-03$ & $S m-153$ & $7 E-09$ & $5 E-05$ \\
\hline La-137 & $2 E-10$ & $3 E-04$ & $\mathrm{Sm}-155$ & $5 E-07$ & $2 E-03$ \\
\hline La-138 & $8 E-12$ & $2 E-05$ & $S m-156$ & $2 E-08$ & $1 E-04$ \\
\hline La-140 & $4 E-09$ & $2 E-05$ & $E u-145$ & $5 E-09$ & $4 E-05$ \\
\hline La-141 & $2 E-08$ & $1 E-04$ & $E u-146$ & $3 E-09$ & $3 E-05$ \\
\hline La-142 & $5 E-08$ & $2 E-04$ & $E u-147$ & $4 E-09$ & $8 E-05$ \\
\hline$L a-143$ & $2 E-07$ & $1 E-03$ & $E u-148$ & $8 E-10$ & $3 E-05$ \\
\hline $\mathrm{C}_{\theta}-134$ & $2 E-09$ & $1 E-05$ & Eu-149 & $7 E-09$ & $3 E-04$ \\
\hline $\mathrm{Ce}-135$ & $8 E-09$ & $4 E-05$ & $E u-150$ & $2 E-08$ & $9 E-05$ \\
\hline $\mathrm{Ce}-137 \mathrm{~m}$ & $9 E-09$ & $7 E-05$ & $E u-150 m$ & $4 E-11$ & $2 E-05$ \\
\hline $\mathrm{C}_{\theta-137}$ & $3 E-07$ & $1 E-03$ & $E u-152 m$ & $2 E-08$ & $7 E-05$ \\
\hline $\mathrm{C} \theta-139$ & $2 E-09$ & $1 E-04$ & $E u-152$ & $6 E-11$ & $2 E-05$ \\
\hline $\mathrm{C}_{\theta}-141$ & $1 E-09$ & $5 E-05$ & Eu-154 & $5 E-11$ & $1 E-05$ \\
\hline $\mathrm{C}_{\theta-143}$ & $4 E-09$ & $3 E-05$ & $E u-155$ & $3 E-10$ & $1 E-04$ \\
\hline$C_{\theta-144}$ & $3 E-11$ & $7 E-06$ & $E u-156$ & $1 E-09$ & $2 E-05$ \\
\hline $\operatorname{Pr}-136$ & $5 E-07$ & $2 E-03$ & $E u-157$ & $1 E-08$ & $6 E-05$ \\
\hline $\operatorname{Pr}-137$ & $3 E-07$ & $1 E-03$ & $E u-158$ & $1 E-07$ & $5 E-04$ \\
\hline $\mathrm{Pr}-138 \mathrm{~m}$ & $1 E-07$ & $3 E-04$ & $G d-145$ & $4 E-07$ & $1 E-03$ \\
\hline $\operatorname{Pr}-139$ & $2 E-07$ & $1 E-03$ & $G d-146$ & $3 E-10$ & $4 E-05$ \\
\hline $\mathrm{Pr}-142 \mathrm{~m}$ & $3 E-07$ & $2 E-03$ & $G d-147$ & $1 E-08$ & $5 E-05$ \\
\hline $\operatorname{Pr}-142$ & $4 E-09$ & $3 E-05$ & $G d-148$ & $4 E-14$ & $7 E-07$ \\
\hline $\operatorname{Pr}-143$ & $2 E-09$ & $3 E-05$ & $G d-149$ & $5 E-09$ & $8 E-05$ \\
\hline $\operatorname{Pr}-144$ & $3 E-07$ & $1 E-03$ & $G d-151$ & $2 E-09$ & $2 E-04$ \\
\hline $\operatorname{Pr}-145$ & $2 E-08$ & $9 E-05$ & $\mathrm{Gd}-152$ & $5 E-14$ & $9 E-07$ \\
\hline Pr -147 & $4 E-07$ & $2 E-03$ & $G d-153$ & $6 E-10$ & $1 E-04$ \\
\hline & & & $G d-159$ & $2 E-08$ & $7 E-05$ \\
\hline
\end{tabular}

B. 6 
Table B.2. (contd)

\begin{tabular}{|c|c|c|c|c|c|}
\hline \multirow[b]{2}{*}{ Isotope } & \multicolumn{2}{|c|}{$\operatorname{DCG}\left(\mathrm{Cl} / \mathrm{m}^{3}\right)$} & \multirow[b]{2}{*}{ Isotope } & \multicolumn{2}{|c|}{$\mathrm{DCG}\left(\mathrm{Ci} / \mathrm{m}^{3}\right)$} \\
\hline & Air & Water & & Air & Waler \\
\hline $\mathrm{Tb}-147$ & $8 E-08$ & $2 E-04$ & Lu-169 & $1 E-08$ & $7 E-05$ \\
\hline Tb-149 & $2 E-09$ & $1 E-04$ & $L u-170$ & $5 E-09$ & $3 E-05$ \\
\hline$T b-150$ & $5 E-08$ & $1 E-04$ & $L u-171$ & $4 E-09$ & $5 E-05$ \\
\hline Tb-151 & $2 E-08$ & $1 E-04$ & Lu-172 & $3 E-09$ & $3 E-05$ \\
\hline $\mathrm{Tb}-153$ & $2 E-08$ & $1 E-04$ & $L u-173$ & $6 E-10$ & $1 E-04$ \\
\hline Tb-154 & $1 E-08$ & $5 E-05$ & $L u-174 m$ & $5 E-10$ & $8 E-05$ \\
\hline Tb-155 & $2 E-08$ & $2 E-04$ & $L u-174$ & $4 E-10$ & $1 E-04$ \\
\hline $\mathrm{Tb}-156 \mathrm{~m}$ & $2 E-08$ & $2 E-04$ & $L u-176 m$ & $5 E-08$ & $2 E-04$ \\
\hline$T b-156$ & $3 E-09$ & $3 E-05$ & $L u-176$ & $2 E-11$ & $2 E-05$ \\
\hline Tb -157 & $1 E-09$ & $1 E-03$ & $L u-177 m$ & $2 E-10$ & $2 E-05$ \\
\hline $\mathrm{Tb}-158$ & $5 E-11$ & $3 E-05$ & $L u-177$ & $5 E-09$ & $7 E-05$ \\
\hline Tb -160 & $5 E-10$ & $2 E-05$ & $\mathrm{Lu}-178 \mathrm{~m}$ & $4 E-07$ & $2 E-03$ \\
\hline$T b-161$ & $4 E-09$ & $5 E-05$ & $L u-178$ & $3 E-07$ & $1 E-03$ \\
\hline Dy -155 & $6 E-08$ & $2 E-04$ & Lu-179 & $4 E-08$ & $2 E-04$ \\
\hline Dy -157 & $2 E-07$ & $5 E-04$ & $H f-170$ & $1 E-08$ & $7 E-05$ \\
\hline Dy -159 & $6 E-09$ & $3 E-04$ & $H f-172$ & $4 E-11$ & $3 E-05$ \\
\hline$D y-165$ & $1 E-07$ & $4 E-04$ & $H f-173$ & $3 E-08$ & $1 E-04$ \\
\hline Dy-166 & $2 E-09$ & $2 E-05$ & $H f-175$ & $2 E-09$ & $8 E-05$ \\
\hline $\mathrm{Ho}-155$ & $4 E-07$ & $1 E-03$ & $H f-177 m$ & $1 E-07$ & $5 E-04$ \\
\hline Ho- 157 & $3 E-06$ & $7 E-03$ & $\mathrm{Ht}-178 \mathrm{~m}$ & $6 E-12$ & $7 E-06$ \\
\hline $\mathrm{Ho}-159$ & $2 E-06$ & $6 E-03$ & $H I-179 m$ & $1 E-09$ & $3 E-05$ \\
\hline Ho-161 & $1 E-06$ & $3 E-03$ & $\mathrm{Hf}-180 \mathrm{~m}$ & $5 E-08$ & $2 E-04$ \\
\hline $\mathrm{Ho}-162 \mathrm{~m}$ & $6 E-07$ & $2 E-03$ & $H f-181$ & $9 E-10$ & $3 E-05$ \\
\hline Ho-162 & $6 E-06$ & $2 E-02$ & $\mathrm{HF}-182 m$ & $2 E-07$ & $1 E-03$ \\
\hline $\mathrm{Ho}-164 \mathrm{~m}$ & $7 E-07$ & $3 E-03$ & $H f-182$ & $4 E-12$ & $1 E-05$ \\
\hline Ho-164 & $2 E-06$ & $6 E-03$ & $H f-183$ & $1 E-07$ & $5 E-04$ \\
\hline $\mathrm{Ho}-166 \mathrm{~m}$ & $2 E-11$ & $2 E-05$ & $H f-184$ & $2 E-08$ & $7 E-05$ \\
\hline Ho-166 & $4 E-09$ & $2 E-05$ & $\mathrm{Ta}-172$ & $2 E-07$ & $1 E-03$ \\
\hline $\mathrm{Ho}-167$ & $1 E-07$ & $4 E-04$ & $\mathrm{Ta}-173$ & $4 E-08$ & $2 E-04$ \\
\hline$E r-161$ & $2 E-07$ & $4 E-04$ & $T a-174$ & $2 E-07$ & $7 E-04$ \\
\hline$E r-165$ & $4 E-07$ & $2 E-03$ & $T a-175$ & $3 E-08$ & $2 E-04$ \\
\hline$E r-169$ & $6 E-09$ & $1 E-04$ & $\mathrm{Ta}-176$ & $3 E-08$ & $1 E-04$ \\
\hline$E r-171$ & $2 E-08$ & $1 E-04$ & $\mathrm{Ta}-177$ & $4 E-08$ & $3 E-04$ \\
\hline$E r-172$ & $3 E-09$ & $4 E-05$ & $\mathrm{Ta}-178$ & $2 E-07$ & $5 E-04$ \\
\hline$T m-162$ & $7 E-07$ & $2 E-03$ & $\mathrm{Ta}-179$ & $2 E-09$ & $6 E-04$ \\
\hline$T m-166$ & $3 E-08$ & $1 E-04$ & $\mathrm{Ta}-180 \mathrm{~m}$ & $1 E-07$ & $6 E-04$ \\
\hline$T m-167$ & $5 E-09$ & $7 E-05$ & $\mathrm{Ta}-180$ & $6 E-11$ & $4 E-05$ \\
\hline$T m-170$ & $5 E-10$ & $3 E-05$ & $\mathrm{Ta}-182 \mathrm{~m}$ & $1 E-06$ & $6 E-03$ \\
\hline$T m-171$ & $1 E-09$ & $3 E-04$ & $T a-182$ & $3 E-10$ & $2 E-05$ \\
\hline$T m-172$ & $3 E-09$ & $2 E-05$ & $T a-183$ & $2 E-09$ & $3 E-05$ \\
\hline$T m-173$ & $3 E-08$ & $1 E-04$ & $\mathrm{Ta}-184$ & $1 E-08$ & $5 E-05$ \\
\hline$T m-175$ & $6 E-07$ & $2 E-03$ & $T a-185$ & $2 E-07$ & $7 E-04$ \\
\hline$Y b-162$ & $7 E-07$ & $2 E-03$ & $\mathrm{Ta}-186$ & $5 E-07$ & $2 E-03$ \\
\hline$Y b-166$ & $4 E-09$ & $4 E-05$ & $W-176$ & $1 E-07$ & $3 E-04$ \\
\hline$Y b-167$ & $2 E-06$ & $8 E-03$ & $W-177$ & $2 E-07$ & $6 E-04$ \\
\hline$Y b-169$ & $2 E-09$ & $5 E-05$ & $W-178$ & $5 E-08$ & $2 E-04$ \\
\hline$Y b-175$ & $8 E-09$ & $9 E-05$ & $W-179$ & $4 E-06$ & $1 E-02$ \\
\hline$Y b-177$ & $1 E-07$ & $4 E-04$ & $W-181$ & $8 E-08$ & $4 E-04$ \\
\hline$Y b-178$ & $9 E-08$ & $3 E-04$ & $W-185$ & $2 E-08$ & $7 E-05$ \\
\hline & & & $W-187$ & $2 E-08$ & $5 E-05$ \\
\hline & & & $W-188$ & $3 E-09$ & $1 E-05$ \\
\hline
\end{tabular}

B. 7 
Table B.2. (contd)

\begin{tabular}{|c|c|c|c|c|c|c|}
\hline \multirow[b]{2}{*}{ Isotope } & \multicolumn{2}{|c|}{$\mathrm{DCG}\left(\mathrm{Ci} / \mathrm{m}^{3}\right)$} & & \multirow[b]{2}{*}{ Isotope } & \multicolumn{2}{|c|}{$\mathrm{DCG}\left(\mathrm{Ci} / \mathrm{m}^{3}\right)$} \\
\hline & Air & Water & & & Air & Water \\
\hline $\begin{array}{l}R \theta-177 \\
R \theta-178\end{array}$ & $\begin{array}{l}8 E-07 \\
7 E-07\end{array}$ & $\begin{array}{l}3 E-03 \\
3 E-03\end{array}$ & & $\begin{array}{l}A U-198 m \\
A U-198\end{array}$ & $\begin{array}{l}3 E-09 \\
4 E-09\end{array}$ & $\begin{array}{l}3 E-05 \\
3 E-05\end{array}$ \\
\hline $\mathrm{Re}-181$ & $2 E-08$ & $1 E-04$ & & $A u-199$ & $9 E-09$ & $9 E-05$ \\
\hline$R \theta-182$ & $5 E-09$ & $4 E-05$ & & $\mathrm{Au}-200 \mathrm{~m}$ & $6 E-09$ & $3 E-05$ \\
\hline$R e-182 m$ & $4 E-08$ & $2 E-04$ & & $\mathrm{Au}-200$ & $2 E-07$ & $7 E-04$ \\
\hline$R \theta-184 m$ & $1 E-09$ & $6 E-05$ & & $A u-201$ & $5 E-07$ & $2 E-03$ \\
\hline$R \theta-184$ & $3 E-09$ & $6 E-05$ & & $\mathrm{Hg}-193 \mathrm{~m}$ & $2 E-08$ & $9 E-05$ \\
\hline$R_{\theta}-186 m$ & $4 E-10$ & $4 E-05$ & & $\mathrm{Hg}-193$ & $1 E-07$ & $4 E-04$ \\
\hline$R \theta-186$ & $4 E-09$ & $5 E-05$ & & $\mathrm{Hg}-194$ & $1 E-10$ & $2 E-05$ \\
\hline$R \theta-187$ & $2 E-07$ & $2 E-02$ & & $\mathrm{Hg}-195 \mathrm{~m}$ & $1 E-08$ & $6 E-05$ \\
\hline$R e-188 m$ & $3 E-07$ & $2 E-03$ & & $\mathrm{Hg}-195$ & $8 E-08$ & $4 E-04$ \\
\hline$R \theta-188$ & $7 E-09$ & $5 E-05$ & & $\mathrm{Hg}-197 \mathrm{~m}$ & $2 E-08$ & $8 E-05$ \\
\hline$R \theta-189$ & $1 E-08$ & $9 E-05$ & & $\mathrm{Hg}-197$ & $3 E-08$ & $2 E-04$ \\
\hline Os-180 & $9 E-07$ & $3 E-03$ & & $\mathrm{Hg}-199 \mathrm{~m}$ & $3 E-07$ & $2 E-03$ \\
\hline$O s-181$ & $1 E-07$ & $4 E-04$ & & $\mathrm{Hg}-203$ & $3 E-09$ & $7 E-05$ \\
\hline Os -182 & $1 E-08$ & $6 E-05$ & & $\mathrm{Tl}-194 \mathrm{~m}$ & $4 E-07$ & $2 E-03$ \\
\hline Os-185 & $1 E-09$ & $7 E-05$ & & $T I-194$ & $1 E-06$ & $7 E-03$ \\
\hline Os $-189 m$ & $5 E-07$ & $2 E-03$ & & $T I-195$ & $3 E-07$ & $2 E-03$ \\
\hline Os-191m & $7 E-08$ & $4 E-04$ & & $T I-197$ & $3 E-07$ & $2 E-03$ \\
\hline Os-191 & $5 E-09$ & $7 E-05$ & & $\mathrm{TI}-198 \mathrm{~m}$ & $1 E-07$ & $8 E-04$ \\
\hline Os -193 & $1 E-08$ & $4 E-05$ & & $T I-198$ & $7 E-08$ & $5 E-04$ \\
\hline Os-194 & $1 E-10$ & $1 E-05$ & & $T 1-199$ & $2 E-07$ & $2 E-03$ \\
\hline $\mid r-182$ & $3 E-07$ & $1 E-03$ & . & $T I-200$ & $3 E-08$ & $2 E-04$ \\
\hline $\mid r-184$ & $6 E-08$ & $2 E-04$ & & $T I-201$ & $5 E-08$ & $5 E-04$ \\
\hline $\mid r-185$ & $2 E-08$ & $1 E-04$ & & $T 1-202$ & $1 E-08$ & $9 E-05$ \\
\hline $1 \mathrm{r}-186$ & $1 E-08$ & $7 E-05$ & & $\mathrm{TI}-204$ & $5 E-09$ & $4 E-05$ \\
\hline $1 \mathrm{r}-187$ & $6 E-08$ & $3 E-04$ & & $\mathrm{~Pb}-195 \mathrm{~m}$ & $5 E-07$ & $2 E-03$ \\
\hline $1 r-188$ & $8 E-09$ & $5 E-05$ & & $P b-198$ & $2 E-07$ & $9 E-04$ \\
\hline $\mid r-189$ & $9 E-09$ & $2 E-04$ & & $\mathrm{~Pb}-199$ & $2 E-07$ & $6 E-04$ \\
\hline $\mid r-190 m$ & $4 E-07$ & $5 E-03$ & & $\mathrm{~Pb}-200$ & $1 E-08$ & $9 E-05$ \\
\hline $\mid r-190$ & $2 E-09$ & $3 E-05$ & & $P b-201$ & $5 E-08$ & $2 E-04$ \\
\hline ir $-192 m$ & $4 E-11$ & $9 E-05$ & & $\mathrm{~Pb}-202 \mathrm{~m}$ & $7 E-08$ & $3 E-04$ \\
\hline $\mid r-192$ & $5 E-10$ & $3 E-05$ & & $P b-202$ & $1 E-10$ & $4 E-06$ \\
\hline $\mid r-194 m$ & $2 E-10$ & $2 E-05$ & & $\mathrm{~Pb}-203$ & $2 E-08$ & $1 E-04$ \\
\hline $\mid r-194$ & $4 E-09$ & $3 E-05$ & & $P b-205$ & $3 E-09$ & $9 E-05$ \\
\hline $\mid r-195 m$ & $5 E-08$ & $2 E-04$ & & $\mathrm{~Pb}-209$ & $1 E-07$ & $7 E-04$ \\
\hline Ir-195 & $1 E-07$ & $4 E-04$ & & $P b-210$ & $9 E-13$ & $3 E-08$ \\
\hline$P t-186$ & $9 E-08$ & $4 E-04$ & & $P b-211$ & $2 E-09$ & $3 E-04$ \\
\hline$P t-188$ & $4 E-09$ & $5 E-05$ & & $\mathrm{~Pb}-212$ & $8 E-11$ & $3 E-06$ \\
\hline$P t-189$ & $7 E-08$ & $3 E-04$ & & $P b-214$ & $2 E-09$ & $2 E-04$ \\
\hline$P t-191$ & $2 E-08$ & $1 E-04$ & & $B \mid-200$ & $2 E-07$ & $8 E-04$ \\
\hline$P t-193 m$ & $1 E-08$ & $8 E-05$ & & $B i-201$ & $9 E-08$ & $3 E-04$ \\
\hline$P t-193$ & $6 E-08$ & $1 E-03$ & & $B i-202$ & $2 E-07$ & $4 E-04$ \\
\hline Pt-195m & $1 E-08$ & $6 E-05$ & & $B i-203$ & $1 E-08$ & $7 E-05$ \\
\hline$P t-197 m$ & $1 E-07$ & $4 E-04$ & - & $B 1-205$ & $3 E-09$ & $4 E-05$ \\
\hline$P t-197$ & $2 E-08$ & $9 E-05$ & & $B \mid-206$ & $2 E-09$ & $2 E-05$ \\
\hline$P t-199$ & $3 E-07$ & $1 E-03$ & & $B 1-207$ & $8 E-10$ & $3 E-05$ \\
\hline$P t-200$ & $8 E-09$ & $3 E-05$ & & $\mathrm{Bl}-210 \mathrm{~m}$ & $2 E-12$ & $2 E-06$ \\
\hline $\mathrm{Au}-193$ & $4 E-08$ & $3 E-04$ & & $B i-210$ & $6 E-11$ & $2 E-05$ \\
\hline $\mathrm{Au}-194$ & $1 E-08$ & $8 E-05$ & & $B i-212$ & $7 E-10$ & $1 E-04$ \\
\hline $\mathrm{Au}-195$ & $1 E-09$ & $1 E-04$ & & $\begin{array}{l}8 i-213 \\
8 i-214\end{array}$ & $\begin{array}{l}8 E-10 \\
2 E-09\end{array}$ & $\begin{array}{l}2 E-04 \\
6 E-04\end{array}$ \\
\hline
\end{tabular}

B. 8 
Table B.2. (contd)

\begin{tabular}{|c|c|c|c|c|c|c|}
\hline \multirow{2}{*}{ Isotope } & \multicolumn{2}{|c|}{ DCG $\left(\mathrm{Ci} / \mathrm{m}^{3}\right)$} & & \multirow[b]{2}{*}{ Isotope } & \multicolumn{2}{|c|}{$\mathrm{DCG}\left(\mathrm{Ci} / \mathrm{m}^{3}\right)$} \\
\hline & Air & Water & & & Air & Water \\
\hline Po-203 & $2 E-07$ & $7 E-04$ & & $N p-232$ & $1 E \sim 08$ & $4 E-03$ \\
\hline Po-205 & $2 E-07$ & $6 E-04$ & & $N p-233$ & $7 E-06$ & $2 E-02$ \\
\hline Po-207 & $7 E-08$ & $2 E-04$ & & $N p-234$ & $6 E-09$ & $6 E-05$ \\
\hline Po-210 & $1 E-12$ & $8 E-08$ & & $N p-235$ & $3 E-09$ & $7 E-04$ \\
\hline At-207 & $5 E-09$ & $2 E-04$ & & $N p-236$ & $1 E-13$ & $2 E-07$ \\
\hline At -211 & $1 E-10$ & $3 E-06$ & & $N p-236 m$ & $2 E-10$ & $1 E-04$ \\
\hline $\mathrm{An}-220$ & $3 E-09$ & $3 E-09$ & & $N p-237$ & $2 E-14$ & $3 E-08$ \\
\hline $\mathrm{An}-222$ & $3 E-09$ & $3 E-09$ & & $N p-238$ & $4 E-10$ & $4 E-05$ \\
\hline$F r-222$ & $1 E-09$ & $6 E-05$ & & $N p-239$ & $5 E-09$ & $5 E-05$ \\
\hline $\mathrm{Fr}-223$ & $2 E-09$ & $2 E-05$ & & $N p-240$ & $2 E-07$ & $6 E-04$ \\
\hline $\mathrm{Ra}-223$ & $2 E-12$ & $2 E-07$ & & $P u-234$ & $4 E-10$ & $3 E-04$ \\
\hline $\mathrm{Ra}-224$ & $4 E-12$ & $4 E-07$ & & $P u-235$ & $6 E-06$ & $2 E-02$ \\
\hline $\mathrm{Ra}-225$ & $2 E-12$ & $4 E-07$ & & $P u-236$ & $1 E-13$ & $5 E-06$ \\
\hline$R a-226$ & $1 E-12$ & $1 E-07$ & & $P u-237$ & $7 E-09$ & $3 E-04$ \\
\hline $\mathrm{Ra}-227$ & $4 E-0 B$ & $6 E-04$ & & $P u-238$ & $4 E-14$ & $5 E-06$ \\
\hline $\mathrm{Ra}-228$ & $3 E-12$ & $1 E-07$ & & $P u-239$ & $4 E-14$ & $4 E-06$ \\
\hline$A C-224$ & $1 E-10$ & $5 E-05$ & & $P u-240$ & $4 E-14$ & $4 E-06$ \\
\hline$A C-225$ & $1 E-12$ & $1 E-06$ & & $P u-241$ & $2 E-12$ & $2 E-04$ \\
\hline$A C-226$ & $1 E-11$ & $4 E-06$ & & $P u-242$ & $4 E-14$ & $5 E-06$ \\
\hline$A C-227$ & $1 E-14$ & $1 E-08$ & & $P u-243$ & $9 E-08$ & $4 E-04$ \\
\hline$A C-228$ & $1 E-10$ & $6 E-05$ & & $P u-244$ & $4 E-14$ & $3 E-06$ \\
\hline$T h-226$ & $3 E-10$ & $2 E-04$ & & $P u-245$ & $1 E-08$ & $6 E-05$ \\
\hline$T h-227$ & $7 E-13$ & $4 E-06$ & . & $P u-246$ & $6 E-10$ & $1 E-05$ \\
\hline Th-228 & $4 E-14$ & $4 E-07$ & . & $A m-237$ & $7 E-07$ & $2 E-03$ \\
\hline$T h-229$ & $7 E-15$ & $4 E-08$ & & $A m-238$ & $1 E-08$ & $1 E-03$ \\
\hline$T h-230$ & $5 E-14$ & $3 E-07$ & & $A m-239$ & $3 E-08$ & $1 E-04$ \\
\hline$T h-231$ & $1 E-08$ & $1 E-04$ & . & Am -240 & $7 E-09$ & $6 E-05$ \\
\hline$T h-232$ & $1 E-14$ & $5 E-08$ & & $A m-241$ & $3 E-14$ & $4 E-08$ \\
\hline$T h-234$ & $4 E-10$ & $1 E-05$ & & $\mathrm{Am}-242 \mathrm{~m}$ & $3 E-14$ & $4 E-08$ \\
\hline $\mathrm{Pa}-227$ & $2 E-10$ & $1 E-04$ & & Am-242 & $2 E-10$ & $1 E-04$ \\
\hline $\mathrm{Pa}-228$ & $3 E-11$ & $3 E-05$ & & $A m-243$ & $3 E-14$ & $4 E-08$ \\
\hline $\mathrm{Pa}-230$ & $8 E-12$ & $2 E-05$ & & $A m-244 m$ & $2 E-08$ & $2 E-03$ \\
\hline $\mathrm{Pa}-231$ & $1 E-14$ & $1 E-08$ & & $A m-244$ & $7 E-10$ & $7 E-05$ \\
\hline $\mathrm{Pa}-232$ & $2 E-10$ & $4 E-05$ & & $A m-245$ & $2 E-07$ & $8 E-04$ \\
\hline $\mathrm{Pa}-233$ & $1 E-09$ & $4 E-05$ & & $A m-246 m$ & $5 E-07$ & $2 E-03$ \\
\hline $\mathrm{Pa}-234$ & $2 E-08$ & $7 E-05$ & & $A m-246$ & $2 E-07$ & $9 E-04$ \\
\hline$U-230$ & $6 E-13$ & $1 E-06$ & & $\mathrm{Cm}-238$ & $3 E-09$ & $4 E-04$ \\
\hline$U-231$ & $1 E-08$ & $1 E-04$ & & $\mathrm{Cm}-240$ & $1 E-12$ & $2 E-06$ \\
\hline$U-232$ & $2 E-14$ & $2 E-06$ & & $\mathrm{Cm}-241$ & $8 E-11$ & $3 E-05$ \\
\hline$U-233$ & $9 E-14$ & $5 E-06$ & & $\mathrm{Cm}-242$ & $7 E-13$ & $1 E-06$ \\
\hline$U-234$ & $9 E-14$ & $6 E-06$ & & $\mathrm{Cm}-243$ & $4 E-14$ & $6 E-08$ \\
\hline$U-235$ & $9 E-14$ & $5 E-06$ & & $\mathrm{Cm}-244$ & $5 E-14$ & $7 E-08$ \\
\hline$U-236$ & $9 E-14$ & $6 E-06$ & & $\mathrm{Cm}-245$ & $3 E-14$ & $4 E-08$ \\
\hline$U-237$ & $4 E-09$ & $5 E-05$ & & $\mathrm{Cm}-246$ & $3 E-14$ & $4 E-08$ \\
\hline$U-238$ & $1 E-13$ & $6 E-06$ & & $\mathrm{Cm}-247$ & $3 E-14$ & $4 E-08$ \\
\hline$U-239$ & $4 E-07$ & $2 E-03$ & & $\mathrm{Cm}-248$ & $7 E-15$ & $1 E-08$ \\
\hline$U-240$ & $6 E-09$ & $3 E-05$ & & $\mathrm{Cm}-249$ & $6 E-08$ & $1 E-03$ \\
\hline & & & & $\mathrm{Cm}-250$ & $1 E-15$ & $2 E-09$ \\
\hline
\end{tabular}


Table B.2. (contd)

\begin{tabular}{|c|c|c|}
\hline \multirow[b]{2}{*}{ Isotope } & \multicolumn{2}{|c|}{$\operatorname{DCG}\left(\mathrm{Cl} / \mathrm{m}^{3}\right)$} \\
\hline & Air & Water \\
\hline$k-245$ & $3 E-09$ & $6 E-05$ \\
\hline $3 k-246$ & $7 E-09$ & $7 E-05$ \\
\hline $3 k-247$ & $2 E-14$ & $3 E-08$ \\
\hline$B k-249$ & $9 E-12$ & $1 E-05$ \\
\hline$B K-250$ & $2 E-09$ & $2 E-04$ \\
\hline$C f-244$ & $1 E-09$ & $8 E-04$ \\
\hline$C f-246$ & $2 E-11$ & $1 E-05$ \\
\hline$C f-248$ & $3 E-13$ & $5 E-07$ \\
\hline$C f-249$ & $2 E-14$ & $3 E-08$ \\
\hline$C f-250$ & $5 E-14$ & $7 E-08$ \\
\hline $\mathrm{Ct}-251$ & $2 E-14$ & $3 E-08$ \\
\hline$C f-252$ & $9 E-14$ & $1 E-07$ \\
\hline $\mathrm{Cl}-253$ & $5 E-12$ & $1 E-05$ \\
\hline$C f-254$ & $5 E-14$ & $6 E-08$ \\
\hline$E s-250$ & $3 E-09$ & $1 E-03$ \\
\hline Es-251 & $3 E-09$ & $2 E-04$ \\
\hline Es-253 & $3 E-12$ & $4 E-06$ \\
\hline Es-254m & $2 E-11$ & $9 E-06$ \\
\hline Es-254 & $3 E-13$ & $5 E-07$ \\
\hline $\mathrm{Fm}-252$ & $3 E-11$ & $1 E-05$ \\
\hline$F m-253$ & $2 E-11$ & $3 E-05$ \\
\hline $\mathrm{Fm}-254$ & $2 E-10$ & $8 E-05$ \\
\hline$F m-255$ & $5 E-11$ & $1 E-05$ \\
\hline $\mathrm{Fm}-257$ & $5 E-13$ & $1 E-06$ \\
\hline
\end{tabular}

B. 10 
Table B.3. Dose-Rate Factors for Skin for Exposure $1 \mathrm{~m}$ Above Contaminated Ground Surface, $\mathrm{mrem} / \mathrm{yr} / \mu \mathrm{Ci} / \mathrm{m}^{2}$

\begin{tabular}{|c|c|c|c|c|}
\hline Isotope & Half-Life & Photon & Electron & Total \\
\hline H 3 & $12.28 \mathrm{Y}$ & 0 & 0 & \\
\hline BE 7 & $53.44 \mathrm{D}$ & 6.88 & 0 & 6.88 \\
\hline BE 10 & $1.6 E+06 Y$ & 0 & 15 & 15 \\
\hline C 11 & $20.48 \mathrm{M}$ & 141 & 349 & 490 \\
\hline C 14 & $5730 \mathrm{Y}$ & 0 & 0 & \\
\hline$N 13$ & $9.97 \mathrm{M}$ & 141 & 596 & 737 \\
\hline N 16 & $7.13 \mathrm{~s}$ & 394 & 2110 & 2500 \\
\hline 015 & $122.24 \mathrm{~S}$ & 141 & 1040 & 1180 \\
\hline F 18 & $109.74 \mathrm{M}$ & 136 & 51.7 & 188 \\
\hline NA 22 & $2.602 Y$ & 281 & 14.3 & 295 \\
\hline NA 24 & $15 \mathrm{H}$ & 462 & 724 & 1190 \\
\hline MG 27 & $9.458 \mathrm{M}$ & 120 & 974 & 1090 \\
\hline MG 28 & $20.91 \mathrm{H}$ & 174 & 1.3 & 175 \\
\hline AL 26 & $7.2 E+05 Y$ & 332 & 480 & 812 \\
\hline AL 28 & $2.24 \mathrm{M}$ & 209 & 1550 & 1760 \\
\hline SI 31 & $157.3 \mathrm{M}$ & 0.107 & 801 & 801 \\
\hline SI 32 & $330 \mathrm{Y}$ & 0 & 0 & \\
\hline P 32 & $14.29 \mathrm{D}$ & 0 & 964 & 964 \\
\hline P 33 & $25.4 \mathrm{D}$ & 0 & 0 & \\
\hline S 35 & $87.44 \mathrm{D}$ & 0 & 0 & \\
\hline CL 36 & $3.01 E+05 Y$ & $5.60 \mathrm{E}-05$ & 85.5 & 85.5 \\
\hline $\mathrm{CL} 38$ & $37.21 \mathrm{M}$ & 174 & 1460 & 1630 \\
\hline AR 37 & $35.02 \mathrm{D}$ & 0.00946 & 0 & 0.00946 \\
\hline AR 39 & $269 Y$ & 0 & 27.1 & 27.1 \\
\hline AR 41 & $1.827 \mathrm{H}$ & 155 & 542 & 696 \\
\hline K 40 & $1.28 E+\infty 9 Y$ & 18.2 & 569 & 588 \\
\hline K 42 & $12.36 \mathrm{H}$ & 32.2 & 1620 & 1650 \\
\hline$K 43$ & $22.6 \mathrm{H}$ & 134 & 197 & 332 \\
\hline CA 41 & $1.03 E+05 Y$ & 0.0248 & 0 & 0.0248 \\
\hline CA 45 & $162.7 \mathrm{D}$ & $2.06 E-07$ & 0 & $2.06 E-07$ \\
\hline CA 47 & $4.536 \mathrm{D}$ & 129 & 263 & 392 \\
\hline CA 49 & $8.719 \mathrm{M}$ & 328 & 1180 & 1510 \\
\hline SC 44 & $3.927 \mathrm{H}$ & 279 & 828 & 1110 \\
\hline SC 46 & 83.80 & 262 & 0.0279 & 262 \\
\hline SC $46 \mathrm{~m}$ & $18.72 \mathrm{~S}$ & 12.7 & 0 & 12.7 \\
\hline SC 47 & $3.422 \mathrm{D}$ & 15.3 & 8.73 & 24 \\
\hline SC 48 & $43.67 \mathrm{H}$ & 425 & 47.1 & 472 \\
\hline SC 49 & $57.4 \mathrm{M}$ & 0.122 & 1140 & 1140 \\
\hline TI 44 & $47.3 \mathrm{Y}$ & 21.6 & 0 & 21.6 \\
\hline TI 45 & $3.08 \mathrm{H}$ & 120 & 396 & 516 \\
\hline T1 51 & $5.752 \mathrm{M}$ & 51.1 & 1180 & 1230 \\
\hline V 48 & $15.971 \mathrm{D}$ & 372 & 54.5 & 426 \\
\hline V 49 & $330 \mathrm{D}$ & 0.0905 & 0 & 0.0905 \\
\hline V 52 & $3.75 \mathrm{M}$ & 170 & 1400 & 1560 \\
\hline CR 49 & $42.09 \mathrm{M}$ & 145 & 825 & 970 \\
\hline CR 51 & 27.704 D & 4.53 & 0 & 4.53 \\
\hline MN 52 & $5.591 \mathrm{D}$ & 441 & 8.39 & 450 \\
\hline MN 52m & $21.4 M$ & 303 & 1470 & 1770 \\
\hline MN 53 & 3.7E+06Y & 0.19 & 0 & 0.19 \\
\hline MN 54 & $312.7 \mathrm{D}$ & 114 & 0 & 114 \\
\hline MN 56 & $2.5785 \mathrm{H}$ & 214 & 972 & 1190 \\
\hline MN 57 & $1.47 \mathrm{M}$ & 10.6 & 1420 & 1430 \\
\hline FE 52 & $8.275 \mathrm{H}$ & 102 & 120 & 222 \\
\hline
\end{tabular}

B.11 
Table B.3. (contd)

\begin{tabular}{|c|c|c|c|c|}
\hline Isotope & Half-Life & Photon & Electron & Total \\
\hline FE 55 & $2.7 Y$ & 0.266 & 0 & 0.266 \\
\hline FE 59 & $44.63 \mathrm{D}$ & 148 & 2.07 & 150 \\
\hline CO 56 & $78.76 \mathrm{D}$ & 440 & 164 & 604 \\
\hline Co 57 & $270.9 \mathrm{D}$ & 18.5 & 0 & 18.5 \\
\hline CO 58 & 70.80 & 133 & 0.409 & 134 \\
\hline Co $58 \mathrm{~m}$ & $9.15 \mathrm{H}$ & 0.426 & 0 & 0.426 \\
\hline CO 60 & $5.271 Y$ & 305 & 0 & 305 \\
\hline co $60 \mathrm{~m}$ & $10.47 \mathrm{M}$ & 1.04 & 1.89 & 2.93 \\
\hline CO 61 & $1.65 \mathrm{H}$ & 13.6 & 549 & 562 \\
\hline NI 56 & $6.1 \mathrm{D}$ & 232 & 0 & 232 \\
\hline NI 57 & $36.08 \mathrm{H}$ & 236 & 93.4 & 329 \\
\hline NI 59 & $75,000 \mathrm{Y}$ & 0.5 & 0 & 0.5 \\
\hline NI 63 & $100.1 Y$ & 0 & 0 & 0 \\
\hline $\mathrm{NI} 65$ & $2.52 \mathrm{H}$ & 66.1 & 775 & 841 \\
\hline CU 61 & $3.408 \mathrm{H}$ & 113 & 373 & 486 \\
\hline CU 62 & $9.74 M$ & 139 & 1590. & 1730 \\
\hline CU 64 & $12.701 \mathrm{H}$ & 26.3 & 20.4 & 46.7 \\
\hline CU 67 & $61.88 \mathrm{D}$ & 16.4 & 3.81 & 20.2 \\
\hline ZN 62 & $9.26 \mathrm{H}$ & 64 & 3.46 & 67.5 \\
\hline $\mathrm{ZN} 65$ & $244.4 \mathrm{D}$ & 74.5 & 0 & 74.5 \\
\hline ZN 69 & $55.6 \mathrm{M}$ & $8.34 E-04$ & 236 & 236 \\
\hline ZN 69m & $13.76 \mathrm{H}$ & 57.8 & 8.12 & 65.9 \\
\hline GA 66 & $9.4 \mathrm{H}$ & 293 & 974 & 1270 \\
\hline GA 67 & $3.261 \mathrm{D}$ & 22 & 0 & 22 \\
\hline GA 68 & $68 \mathrm{M}$ & 131 & 1040 & 1170 \\
\hline GA 72 & $14.1 \mathrm{H}$ & 337 & 504 & 842 \\
\hline GE 68 & $288 \mathrm{D}$ & 1.38 & 0 & 1.38 \\
\hline GE 71 & $11.8 \mathrm{D}$ & 1.4 & 0 & 1.4 \\
\hline GE 77 & $11.3 \mathrm{H}$ & 143 & 819 & 962 \\
\hline AS 72 & $26 \mathrm{H}$ & 242 & 1330 & 1570 \\
\hline AS 73 & $80.3 \mathrm{D}$ & 4.72 & 0 & 4.72 \\
\hline AS 74 & $17.77 \mathrm{D}$ & 106 & 277 & 383 \\
\hline AS 76 & $26.32 \mathrm{H}$ & 57.3 & 1330 & 1390 \\
\hline AS 77 & $38.8 \mathrm{H}$ & 1.2 & 64.9 & 66.1 \\
\hline SE 73 & $7.15 \mathrm{H}$ & 153 & 490 & 644 \\
\hline SE 75 & $119.78 \mathrm{D}$ & 56.5 & 0 & 56.5 \\
\hline SE 79 & $65,000 \mathrm{Y}$ & 0 & 0 & 0 \\
\hline BR 77 & $57.04 \mathrm{H}$ & 46 & 0 & 46 \\
\hline BR 80 & $17.4 \mathrm{M}$ & 10.4 & 991 & 1000 \\
\hline BA $80 \mathrm{~m}$ & $4.42 \mathrm{H}$ & 6.54 & 0 & 6.54 \\
\hline BA 82 & $35.3 \mathrm{H}$ & 350 & 0.395 & 350 \\
\hline BR 83 & $2.39 \mathrm{H}$ & 1.03 & 240 & 241 \\
\hline BA 84 & $31.8 \mathrm{M}$ & 208 & 1300 & 1510 \\
\hline BR 85 & $172 \mathrm{~S}$ & 8.79 & 1320 & 1330 \\
\hline KA 79 & $35.04 \mathrm{H}$ & 37.4 & 3.47 & 40.9 \\
\hline KR 81 & 2.1E+05Y & 4.08 & 0 & 4.08 \\
\hline KR 83m & $1.83 \mathrm{H}$ & 1.08 & 0 & 1.08 \\
\hline KR 85 & $10.72 Y$ & 0.308 & 97.9 & 98.2 \\
\hline KR $85 m$ & $4.48 \mathrm{H}$ & 22.5 & 138 & 160 \\
\hline KR 87 & $76.3 \mathrm{M}$ & 96.9 & 1480 & 1580 \\
\hline KR 88 & $2.84 \mathrm{H}$ & 231 & 287 & 519 \\
\hline KR 89 & $3.16 M$ & 220 & 1480 & 1700 \\
\hline KR 90 & $32.32 \mathrm{~S}$ & 160 & 1520 & 1680 \\
\hline
\end{tabular}

B. 12 
Table B.3. (contd)

\begin{tabular}{|c|c|c|c|c|}
\hline Isotope & Half-Life & Photon & Electron & Total \\
\hline RB 81 & $4.58 \mathrm{H}$ & 86.9 & 159 & 246 \\
\hline RB 82 & $1.25 \mathrm{M}$ & 151 & 1640 & 1800 \\
\hline AB 83 & 86.2 D & 72 & 0 & 72 \\
\hline RB 84 & $32.9 \mathrm{D}$ & 124 & 186 & 310 \\
\hline RB 86 & $18.66 \mathrm{D}$ & 12.1 & 905 & 917 \\
\hline RB 87 & 4.73E $+10 Y$ & 0 & 0 & 0 \\
\hline RB 88 & $17.8 \mathrm{M}$ & 76.1 & 1890 & 1960 \\
\hline RB 89 & $15.44 M$ & 253 & 1170 & 1420 \\
\hline RB 90 & $157 \mathrm{~S}$ & 224 & 1760 & 1980 \\
\hline AB 90m & $258 \mathrm{~S}$ & 380 & 1490 & 1870 \\
\hline SR 82 & 250 & 3.53 & 0 & 3.53 \\
\hline SA 85 & $64.84 \mathrm{D}$ & 74 & 3.43 & 77.5 \\
\hline SA $85 \mathrm{~m}$ & $67.66 \mathrm{M}$ & 30.6 & 0 & 30.6 \\
\hline SR $87 m$ & $2.805 \mathrm{H}$ & 45.1 & 1.92 & 47 \\
\hline SR 89 & $50.55 \mathrm{D}$ & 0.0184 & 797 & 797 \\
\hline SR 90 & $28.6 Y$ & 0 & 17.3 & 17.3 \\
\hline SR 91 & $9.5 \mathrm{H}$ & 91.4 & 789 & 880 \\
\hline SR 92 & $2.71 \mathrm{H}$ & 160 & 53.7 & 213 \\
\hline SR 93 & $7.3 \mathrm{M}$ & 288 & 1150 & 1430 \\
\hline$Y 86$ & $14.74 \mathrm{H}$ & 462 & 290 & 751 \\
\hline$Y 87$ & $80.3 \mathrm{H}$ & 66.9 & 0.881 & 67.8 \\
\hline Y 88 & $106.6 \mathrm{D}$ & 334 & 0.523 & 334 \\
\hline$Y 90$ & $64.1 \mathrm{H}$ & 0 & 1250 & 1250 \\
\hline Y 90m & $3.19 \mathrm{H}$ & 88.4 & 30.6 & 119 \\
\hline Y91 & $58.51 \mathrm{D}$ & 0.445 & 829 & 829 \\
\hline Y91m & $49.71 M$ & 73.4 & 37.8 & 111 \\
\hline Y 92 & $3.54 \mathrm{H}$ & 32.6 & 1610 & 1640 \\
\hline Y93 & $10.1 \mathrm{H}$ & 11.3 & 1440 & 1460 \\
\hline ZR 86 & $16.5 \mathrm{H}$ & 46.1 & 0 & 46.1 \\
\hline ZR 88 & $83.4 \mathrm{D}$ & 57.2 & 0.325 & 57.5 \\
\hline ZR 89 & $78.43 \mathrm{H}$ & 159 & 90.3 & 249 \\
\hline ZR 93 & 1.53E+06 Y & 0 & 0 & 0 \\
\hline ZR 95 & 64.020 & 101 & 2.6 & 104 \\
\hline ZR 97 & $16.9 \mathrm{H}$ & 23.2 & 939 & 962 \\
\hline NB 90 & $14.6 \mathrm{H}$ & 509 & 495 & 1000 \\
\hline NB 91 & $10,000 Y$ & 4.12 & 0 & 4.12 \\
\hline NB 91m & $61 D$ & 8.3 & 0 & 8.3 \\
\hline NB $92 m$ & 3.6E+07Y & 206 & 2.01 & 208 \\
\hline NB 92 & 10.150 & 132 & 0 & 132 \\
\hline NB 93m & $14.6 Y$ & 0.66 & 0 & 0.66 \\
\hline NB 94 & $20,300 Y$ & 215 & 3.12 & 218 \\
\hline NB 94m & $6.26 \mathrm{M}$ & 3.07 & 2.4 & 5.47 \\
\hline NB 95 & $35.06 \mathrm{D}$ & 105 & 1.76 & 107 \\
\hline NB 95m & $86.6 \mathrm{H}$ & 10.7 & 29 & 39.6 \\
\hline NB 96 & $23.35 \mathrm{H}$ & 330 & 103 & 433 \\
\hline NB 97 & $72.1 \mathrm{M}$ & 91.6 & 558 & 650 \\
\hline NB 97m & $60 \mathrm{~S}$ & 100 & 25.3 & 125 \\
\hline MO 91 & $15.49 \mathrm{M}$ & 135 & 1660 & 1800 \\
\hline MO 93 & $3500 Y$ & 3.69 & 0 & 3.69 \\
\hline MO 99 & $66.02 \mathrm{H}$ & 21.5 & 422 & 444 \\
\hline MO101 & $14.61 \mathrm{M}$ & 190 & 568 & 758 \\
\hline TC 95 & $20 \mathrm{H}$ & 111 & 1.58 & 113 \\
\hline TC 95m & $61 \mathrm{D}$ & 94.8 & 0.579 & 95.4 \\
\hline
\end{tabular}

B. 13 
Table B.3. (contd)

\begin{tabular}{|c|c|c|c|c|}
\hline Isotope & Half-Life & Photon & Electron & Total \\
\hline TC 96 & $4.28 \mathrm{D}$ & 343 & 1.65 & 344 \\
\hline TC $96 \mathrm{~m}$ & $51.5 \mathrm{M}$ & 7.45 & 0 & 7.45 \\
\hline TC 97 & 2.6E+06 Y & 3.76 & 0 & 3.76 \\
\hline TC $97 \mathrm{~m}$ & $89 \mathrm{D}$ & 2.86 & 0 & 2.86 \\
\hline TC 98 & $4.2 E+06 Y$ & 192 & 4.41 & 196 \\
\hline TC 99 & $2.13 E+05 Y$ & $7.73 E-05$ & 0 & $7.73 E-05$ \\
\hline TC $99 \mathrm{~m}$ & $6.02 \mathrm{H}$ & 18.2 & 0 & 18.2 \\
\hline TC101 & $14.2 \mathrm{M}$ & 47.8 & 561 & 609 \\
\hline RU 97 & 2.90 & 35.7 & 0 & 35.7 \\
\hline RU103 & 39.350 & 66.8 & 4.56 & 71.4 \\
\hline RU105 & $4.44 \mathrm{H}$ & 108 & 422 & 530 \\
\hline RU106 & $368.2 \mathrm{D}$ & 0 & 0 & 0 \\
\hline RH103m & $56.119 \mathrm{M}$ & 0.532 & 0 & 0.532 \\
\hline RH105 & $35.36 \mathrm{H}$ & 10.9 & 10.2 & 21.1 \\
\hline $\mathrm{RH} 105 \mathrm{~m}$ & $45 \mathrm{~S}$ & 6.58 & 0 & 6.58 \\
\hline RH106 & $29.92 \mathrm{~S}$ & 28.2 & 1630 & 1660 \\
\hline PD103 & 16.9610 & 4.73 & 0 & 4.73 \\
\hline PD107 & $6.5 E+06 Y$ & 0 & 0 & 0 \\
\hline P0109 & $13.453 \mathrm{H}$ & 0.095 & 332 & 332 \\
\hline AG106 & $8.46 \mathrm{D}$ & 372 & 2.07 & 374 \\
\hline AG108 & $2.37 \mathrm{M}$ & 2.5 & 824 & 827 \\
\hline AG108m & $127 \mathrm{Y}$ & 226 & 5.18 & 231 \\
\hline AG109m & $39.6 \mathrm{~S}$ & 2.52 & 0 & 2.52 \\
\hline AG110 & $24.57 \mathrm{~S}$ & 4.22 & 1480 & 1480 \\
\hline AG110m & $249.85 \mathrm{D}$ & 361 & 4.77 & 366 \\
\hline AG111 & $7.46 \mathrm{D}$ & 3.68 & 310 & 314 \\
\hline CD109 & $464 \mathrm{D}$ & 3.82 & 0 & 3.82 \\
\hline CD111m & $48.7 \mathrm{M}$ & 40.9 & 0 & 40.9 \\
\hline CD113 & $9.3 E+15 Y$ & 0 & 0 & 0 \\
\hline CD113m & $13.7 Y$ & 0 & 18.3 & 18.3 \\
\hline CD115 & $53.46 \mathrm{H}$ & 28.3 & 253 & 282 \\
\hline CD115m & $44.6 \mathrm{D}$ & 2.84 & 814 & 817 \\
\hline CD117 & $2.49 \mathrm{H}$ & 137 & 454 & 591 \\
\hline CD117m & $3.36 \mathrm{H}$ & 251 & 69.5 & 320 \\
\hline IN111 & $2.83 \mathrm{D}$ & 58.2 & 0 & 58.2 \\
\hline IN113m & $1.658 \mathrm{H}$ & 36.5 & 1.4 & 37.9 \\
\hline IN114 & $71.9 \mathrm{~s}$ & 4.38 & 1060 & 1060 \\
\hline IN114m & $49.51 \mathrm{D}$ & 13.9 & 0 & 13.9 \\
\hline IN115 & $4.6 E+15 Y$ & 0 & 2.51 & 2.51 \\
\hline IN115m & $4.36 \mathrm{H}$ & 23.5 & 6.58 & 30.1 \\
\hline IN116m & $54.15 \mathrm{M}$ & 303 & 224 & 527 \\
\hline IN117 & $43.8 \mathrm{M}$ & 95.7 & 98.2 & 194 \\
\hline IN117m & $116.5 \mathrm{M}$ & 13.1 & 472 & 485 \\
\hline SN113 & $115.1 \mathrm{D}$ & 4.09 & 0 & 4.09 \\
\hline SN117m & $13.6 \mathrm{D}$ & 22.7 & 0 & 22.7 \\
\hline SN119m & $293 \mathrm{D}$ & 2.03 & 0 & 2.03 \\
\hline SN123 & $129.2 \mathrm{D}$ & 0.882 & 700 & 700 \\
\hline SN125 & $9.64 \mathrm{D}$ & 39 & 1060 & 1090 \\
\hline SN126 & $1.0 E+05 Y$ & 8.79 & 0 & 8.79 \\
\hline SB117 & $2.8 \mathrm{H}$ & 26.5 & 0.508 & 27 \\
\hline SB122 & 2.70 & 61 & 730 & 791 \\
\hline SB124 & $60.2 \mathrm{D}$ & 235 & 364 & 599 \\
\hline SB125. & $2.77 \mathrm{Y}$ & 60.2 & 6.89 & 67.1 \\
\hline
\end{tabular}

B. 14 
Table B.3. (contd)

\begin{tabular}{|c|c|c|c|c|}
\hline Isotope & Half-Life & Photon & Electron & Total \\
\hline SB126 & $12.4 \mathrm{D}$ & 379 & 269 & 647 \\
\hline $\mathrm{SB} 126 \mathrm{~m}$ & $19 \mathrm{M}$ & 217 & 796 & 1010 \\
\hline SB127 & $3.85 \mathrm{D}$ & 91.2 & 238 & 329 \\
\hline SB129 & $4.4 \mathrm{H}$ & 189 & 337 & 525 \\
\hline TE121 & $16.8 \mathrm{D}$ & 80.2 & 3.36 & 83.6 \\
\hline TE121m & $154 \mathrm{D}$ & 30.3 & 0 & 30.3 \\
\hline TE123 & $1.0 E+13 \gamma$ & 2.1 & 0 & 2.1 \\
\hline TE123m & $119.7 \mathrm{D}$ & 21.2 & 0 & 21.2 \\
\hline TE125m & $58 \mathrm{D}$ & 5.6 & 0 & 5.6 \\
\hline TE127 & $9.35 \mathrm{H}$ & 0.673 & 62.6 & 63.3 \\
\hline TE127m & 1090 & 1.79 & 2.21 & 4 \\
\hline TE129 & $69.6 \mathrm{M}$ & 7.94 & 667 & 675 \\
\hline TE129m & $33.6 \mathrm{D}$ & 5.6 & 286 & 291 \\
\hline TE131 & $25 \mathrm{M}$ & 57.3 & 921. & 979 \\
\hline TE131m & $30 \mathrm{H}$ & 190 & 58.1 & 248 \\
\hline TE132 & $78.2 \mathrm{H}$ & 33.1 & 0 & 33.1 \\
\hline TE133 & $12.45 \mathrm{M}$ & 122 & 1070 & 1190 \\
\hline TE133m & $55.4 \mathrm{M}$ & 295 & 900 & 1190 \\
\hline TE134 & $41.8 \mathrm{M}$ & 121 & 1.3 & 122 \\
\hline 1122 & $3.62 \mathrm{M}$ & 134 & 1260 & 1400 \\
\hline 1123 & $13.13 \mathrm{H}$ & 24.8 & 0 & 24.8 \\
\hline 1124 & $4.18 \mathrm{D}$ & 140 & 274 & 413 \\
\hline 1125 & $60.14 \mathrm{D}$ & 6.66 & 0 & 6.66 \\
\hline 1126 & $12.93 \mathrm{D}$ & 64.5 & 109 & 174 \\
\hline 1128 & $24.99 \mathrm{M}$ & 10.5 & 1030 & 1040 \\
\hline 1129 & 1.57E +07 Y & 5.28 & 0 & 5.28 \\
\hline 1130 & $12.36 \mathrm{H}$ & 293 & 193 & 486 \\
\hline 1131 & $8.04 \mathrm{D}$ & 53.2 & 22.4 & 75.6 \\
\hline $\mid 132$ & $2.3 \mathrm{H}$ & 308 & 566 & 874 \\
\hline 1133 & $20.8 \mathrm{H}$ & 82.7 & 448 & 531 \\
\hline 1134 & $52.6 \mathrm{M}$ & 348 & 786 & 1130 \\
\hline 1135 & $6.61 \mathrm{H}$ & 193 & 369 & 562 \\
\hline 1136 & $83 \mathrm{~S}$ & 293 & 1930 & 2230 \\
\hline XE122 & $20.1 \mathrm{H}$ & 11.5 & 0 & 11.5 \\
\hline$X E 123$ & $2.14 \mathrm{H}$ & 84.4 & 210 & 295 \\
\hline$X E 125$ & $16.8 \mathrm{H}$ & 38.4 & 0.064 & 38.5 \\
\hline XE127 & 36.406 D & 39.9 & 0 & 39.9 \\
\hline XE129m & $8.89 \mathrm{D}$ & 10.4 & 0 & 10.4 \\
\hline XE131m & $11.84 \mathrm{D}$ & 4.11 & 0 & 4.11 \\
\hline XE133 & $5.245 \mathrm{D}$ & 7.97 & 0 & 7.97 \\
\hline XE133m & 2.190 & 7.13 & 0 & 7.13 \\
\hline XE135 & $9.11 \mathrm{H}$ & 34.6 & 212 & 246 \\
\hline XE135m & $15.36 \mathrm{M}$ & 59.8 & 103 & 163 \\
\hline XE137 & $3.83 \mathrm{M}$ & 25.1 & 1830 & 1850 \\
\hline XE138 & $14.13 \mathrm{M}$ & 138 & 712 & 851 \\
\hline CS126 & $1.64 \mathrm{M}$ & 155 & 1480 & 1640 \\
\hline $\operatorname{cs} 129$ & $32.06 \mathrm{H}$ & 42 & 0.0331 & 42.1 \\
\hline CS131 & $9.688 \mathrm{D}$ & 4.97 & 0 & 4.97 \\
\hline $\operatorname{cs} 132$ & $6.475 \mathrm{D}$ & 100 & 6.07 & 106 \\
\hline CS134 & $2.062 Y$ & 213 & 39.4 & 252 \\
\hline CS134m & $2.9 \mathrm{H}$ & 4.66 & 0 & 4.66 \\
\hline CS135 & $2.3 E+06 Y$ & 0 & 0 & 0 \\
\hline CS136 & $13.16 \mathrm{D}$ & 288 & 6.34 & 295 \\
\hline
\end{tabular}

B. 15 
Table B.3. (contd)

\begin{tabular}{|c|c|c|c|c|}
\hline Isotope & Half-Life & Photon & Electron & Total \\
\hline CS137 & $30.17 \mathrm{Y}$ & 0 & 28.1 & 28.1 \\
\hline $\operatorname{cs} 138$ & $32.2 \mathrm{M}$ & 285 & 1490 & 1780 \\
\hline $\operatorname{cs} 139$ & $9.4 \mathrm{M}$ & 36.2 & 1740 & 1780 \\
\hline BAt31 & $11.8 \mathrm{D}$ & 68.1 & 1.65 & 69.8 \\
\hline BA133 & $10.5 Y$ & 58.2 & 0 & 58.2 \\
\hline BA133m & $38.9 \mathrm{H}$ & 10.6 & 0 & 10.6 \\
\hline BA135m & $28.7 \mathrm{H}$ & 9.6 & 0 & 9.6 \\
\hline $\mathrm{BA} 137 \mathrm{~m}$ & $2.552 M$ & 82.6 & 108 & 191 \\
\hline BA139 & $83.1 \mathrm{M}$ & 4.9 & 1210 & 1210 \\
\hline BA140 & $12.789 \mathrm{D}$ & 27 & 202 & 229 \\
\hline BA141 & $18.27 \mathrm{M}$ & 118 & 1090 & 1210 \\
\hline BA142 & $10.7 \mathrm{M}$ & 118 & 493 & 611 \\
\hline LA140 & $40.22 \mathrm{H}$ & 284 & 665 & 949 \\
\hline LA141 & $3.94 \mathrm{H}$ & 5.06 & 1250 & 1260 \\
\hline LA142 & $95.4 M$ & 320 & 1040 & 1360 \\
\hline CE139 & $137.66 \mathrm{D}$ & 23.9 & 0 & 23.9 \\
\hline CE141 & $32.5 \mathrm{D}$ & 11.1 & 4.88 & 16 \\
\hline CE143 & $33 \mathrm{H}$ & 38.8 & 439 & 478 \\
\hline CE144 & $284.3 \mathrm{D}$ & 2.86 & 0 & 2.86 \\
\hline PR142 & $19.13 \mathrm{H}$ & 6.81 & 1100 & 1110 \\
\hline PR143 & $13.56 \mathrm{D}$ & $1.22 E-06$ & 237 & 237 \\
\hline PR144 & $17.28 \mathrm{M}$ & 3.94 & 1490 & 1490 \\
\hline PR144m & $7.2 \mathrm{M}$ & 2.01 & 0 & 2.01 \\
\hline ND147 & $10.98 \mathrm{D}$ & 20.4 & 108 & 129 \\
\hline ND149 & $1.73 \mathrm{H}$ & 53.6 & 523 & 576 \\
\hline PM143 & $265 \mathrm{D}$ & 44.1 & 1.74 & 45.8 \\
\hline PM144 & $363 \mathrm{D}$ & 215 & 10.7 & 226 \\
\hline PM145 & $17.7 \mathrm{Y}$ & 5.36 & 0 & 5.36 \\
\hline PM146 & $2020 \mathrm{D}$ & 104 & 45.3 & 150 \\
\hline PM147 & $2.6234 \gamma$ & $4.96 E-04$ & 0 & 4.96E-04 \\
\hline PM148 & $5.37 \mathrm{D}$ & 72 & 907 & 979 \\
\hline PM148m & $41.3 \mathrm{D}$ & 273 & 29.1 & 302 \\
\hline PM149 & $53.08 \mathrm{H}$ & 1.62 & 345 & 346 \\
\hline PM151 & $28.4 \mathrm{H}$ & 47.4 & 194 & 241 \\
\hline SM147 & $1.07 E+11 Y$ & 0 & 0 & 0 \\
\hline SM151 & $90 Y$ & 0.00287 & 0 & 0.00287 \\
\hline SM153 & $46.7 \mathrm{H}$ & 10.2 & 68 & 78.2 \\
\hline EU152 & $13.6 \mathrm{Y}$ & 150 & 70.5 & 221 \\
\hline EU152m & $9.32 \mathrm{H}$ & 42.9 & 660 & 702 \\
\hline EU154 & $8.8 Y$ & 162 & 149 & 312 \\
\hline EU155 & $4.96 \mathrm{Y}$ & 9.39 & 0 & 9.39 \\
\hline EU156 & $15.19 \mathrm{D}$ & 167 & 412 & 579 \\
\hline GD152 & $1.1 E+14 Y$ & 0 & 0 & 0 \\
\hline GD153 & $241.6 \mathrm{D}$ & 18.6 & 0 & 18.6 \\
\hline GD159 & $18.56 \mathrm{H}$ & 5.94 & 235 & 241 \\
\hline GD162 & $9.7 \mathrm{M}$ & 59 & 253 & 312 \\
\hline TB157 & $150 \mathrm{Y}$ & 0.896 & 0 & 0.896 \\
\hline TB160 & $72.3 \mathrm{D}$ & 142 & 104 & 246 \\
\hline TB162. & $7.76 \mathrm{M}$ & 150 & 600 & 750 \\
\hline DY157 & $8.06 \mathrm{H}$ & 50.6 & 0 & 50.6 \\
\hline DY165 & $2.334 \mathrm{H}$ & 3.75 & 510 & 514 \\
\hline DY166 & $81.6 \mathrm{H}$ & 6.58 & 0.141 & 6.72 \\
\hline HO166 & $26.8 \mathrm{H}$ & 3.97 & 923 & 927 \\
\hline
\end{tabular}

B. 16 
Table B.3. (contd)

\begin{tabular}{|c|c|c|c|c|}
\hline Isotope & Half-Life & Photon & Electron & Total \\
\hline HO166m & $1200 \mathrm{Y}$ & 220 & 27.5 & 248 \\
\hline ER169 & $9.4 \mathrm{D}$ & 0.00394 & 0 & 0.00394 \\
\hline ER171 & $7.52 \mathrm{H}$ & 53.1 & 337 & 390 \\
\hline TM170 & $128.6 \mathrm{D}$ & 0.902 & 240 & 241 \\
\hline TM171 & $1.92 Y$ & 0.119 & 0 & 0.119 \\
\hline YB169 & $31.97 \mathrm{D}$ & 48.3 & 0 & 48.3 \\
\hline YB175 & $4.19 \mathrm{D}$ & 5.58 & 0.814 & 6.4 \\
\hline LU177 & $6.71 \mathrm{D}$ & 5.05 & 1.93 & 6.99 \\
\hline LU177m & $160.1 \mathrm{D}$ & 141 & 0.105 & 142 \\
\hline HF181 & $42.39 \mathrm{D}$ & 76.2 & 3.21 & 79.4 \\
\hline TA182 & $114.74 \mathrm{D}$ & 165 & 4.32 & 169 \\
\hline W181 & $120.95 \mathrm{D}$ & 6.89 & 0 & 6.89 \\
\hline W185 & $75.1 \mathrm{D}$ & 0.00376 & 0.172 & 0.176 \\
\hline W187 & $23.83 \mathrm{H}$ & 66.4 & 172 & 238 \\
\hline W188 & $69.4 \mathrm{D}$ & 0.249 & 0 & 0.249 \\
\hline RE182 & $64 \mathrm{H}$ & 230 & 0 & 230 \\
\hline RE182m & $12.7 \mathrm{H}$ & 154 & 20.7 & 175 \\
\hline RE183 & $70 \mathrm{D}$ & 24.7 & 0 & 24.7 \\
\hline RE184 & $38 \mathrm{D}$ & 123 & 5.33 & 128 \\
\hline RE184m & $169 \mathrm{D}$ & 55.3 & 0 & 55.3 \\
\hline RE186 & $90.64 \mathrm{H}$ & 3.14 & 293 & 296 \\
\hline RE187 & $4.7 E+10 Y$ & 0 & 0 & 0 \\
\hline RE188 & $16.98 \mathrm{H}$ & 8.08 & 1040 & 1050 \\
\hline OS185 & $93.6 \mathrm{D}$ & 98.9 & 8.53 & 107 \\
\hline OS186 & $2.0 E+15 Y$ & 0 & 0 & 0 \\
\hline OS190m & $9.9 \mathrm{M}$ & 221 & 16 & 237 \\
\hline OS191 & $15.4 \mathrm{D}$ & 11.9 & 0 & 11.9 \\
\hline OS191m & $13.03 \mathrm{H}$ & 1.37 & 0 & 1.37 \\
\hline OS193 & $30 \mathrm{H}$ & 9.79 & 313 & 323 \\
\hline IR190 & $11.78 \mathrm{D}$ & 195 & 7.23 & 202 \\
\hline IRt90m & $1.2 \mathrm{H}$ & 0.688 & 0 & 0.688 \\
\hline IR190m & $3.2 \mathrm{H}$ & 8.26 & 0 & 8.26 \\
\hline IR192 & $74.02 \mathrm{D}$ & 114 & 28.5 & 143 \\
\hline IR193m & $11.9 \mathrm{D}$ & 0.724 & 0 & 0.724 \\
\hline IR194 & $19.15 \mathrm{H}$ & 12.3 & 1080 & 1100 \\
\hline IR194m & 1710 & 324 & 21.9 & 346 \\
\hline PT191 & $2.71 \mathrm{D}$ & 43.2 & 2.03 & 45.2 \\
\hline PT193 & $50 Y$ & 0.671 & 0 & 0.671 \\
\hline PT193m & $4.33 \mathrm{D}$ & 2.47 & 0 & 2.47 \\
\hline PT195m & $4.02 \mathrm{D}$ & 13 & 0 & 13 \\
\hline PT197 & $18.3 \mathrm{H}$ & 4.1 & 35.5 & 39.6 \\
\hline PT197m & $94.4 \mathrm{M}$ & 13.3 & 2.15 & 15.4 \\
\hline AU194 & $39.5 \mathrm{H}$ & 139 & 14.4 & 153 \\
\hline AU195 & $183 \mathrm{D}$ & 14.4 & 0 & 14.4 \\
\hline AU195m & $30.6 \mathrm{~S}$ & 29 & 0 & 29 \\
\hline AU196 & $6.183 \mathrm{D}$ & 67.6 & 0 & 67.6 \\
\hline AU198 & $2.696 \mathrm{D}$ & 56.3 & 239 & 296 \\
\hline AU199 & 3.1390 & 13 & 0.0612 & 13.1 \\
\hline HG197 & $64.14 \mathrm{H}$ & 11.9 & 0 & 11.9 \\
\hline HG197m & $23.8 \mathrm{H}$ & 14.7 & 0 & 14.7 \\
\hline HG203 & $46.6 \mathrm{D}$ & 32.2 & 0 & 32.2 \\
\hline$\pi 200$ & $26.1 \mathrm{H}$ & 173 & 10.6 & 183 \\
\hline TL201 & $73.06 \mathrm{H}$ & 15.1 & 0 & 15.1 \\
\hline
\end{tabular}

B. 17 
Table B.3． (contd)

\begin{tabular}{|c|c|c|c|c|}
\hline Isotope & Half-Life & Photon & Electron & Total \\
\hline TL202 & $12.23 \mathrm{D}$ & 66.5 & 1.54 & 68.1 \\
\hline TL204 & $3.779 \mathrm{Y}$ & 0.191 & 135 & 135 \\
\hline TL207 & $4.77 \mathrm{M}$ & 0.293 & 613 & 614 \\
\hline TL208 & $3.053 \mathrm{M}$ & 394 & 739 & 1130 \\
\hline TL209 & $2.2 \mathrm{M}$ & 260 & 902 & 1160 \\
\hline TL210 & $1.3 \mathrm{M}$ & 351 & 884 & 1230 \\
\hline PB203 & $52.02 \mathrm{H}$ & 44.3 & 0 & 44.3 \\
\hline PB204m & $66.9 \mathrm{M}$ & 283 & 136 & 419 \\
\hline PB205 & $1.51 E+07 Y$ & 0.882 & 0 & 0.882 \\
\hline PB209 & $3.253 \mathrm{H}$ & 0 & 34.7 & 34.7 \\
\hline PB210 & $22.26 Y$ & 1.37 & 0 & 1.37 \\
\hline PB211 & $36.1 \mathrm{M}$ & 7.01 & 538 & 545 \\
\hline PB212 & $10.643 \mathrm{H}$ & 21.4 & 1.28 & 22.7 \\
\hline PB214 & $26.8 \mathrm{M}$ & 35.3 & 72.3 & 108 \\
\hline $\mathrm{B} 1206$ & $6.243 \mathrm{D}$ & 435 & 52 & 487 \\
\hline B1207 & $33.4 \mathrm{Y}$ & 204 & 162 & 366 \\
\hline B1208 & $3.68 E+05 Y$ & $295^{\circ}$ & 3.63 & 299 \\
\hline B1210 & $5.013 \mathrm{D}$ & 0 & 403 & 403 \\
\hline $\mathrm{B} \mid 211$ & $2.13 \mathrm{M}$ & 6.67 & 0.039 & 6.71 \\
\hline $\mathrm{B} 1212$ & $60.55 \mathrm{M}$ & 24.2 & 603 & 627 \\
\hline 81213 & $45.65 \mathrm{M}$ & 19.4 & 475 & 494 \\
\hline Bl214 & $19.9 M$ & 188 & 787 & 974 \\
\hline PO209 & $102 Y$ & 0.48 & 0 & 0.48 \\
\hline PO210 & $138.378 \mathrm{D}$ & 0.00116 & 0 & 0.00116 \\
\hline PO211 & $0.516 \mathrm{~S}$ & 1.06 & 0 & 1.06 \\
\hline PO212 & $2.98 E-07 \mathrm{~S}$ & 0 & 0 & 0 \\
\hline PO213 & $4.2 E-06 S$ & 0.00418 & 0 & 0.00418 \\
\hline PO214 & $1.637 E-04 S$ & 0.0114 & 0 & 0.0114 \\
\hline PO215 & $1.778 E-03 S$ & 0.0207 & 0 & 0.0207 \\
\hline PO216 & $0.146 \mathrm{~S}$ & 0.00198 & 0 & 0.00198 \\
\hline PO218 & $3.05 \mathrm{M}$ & 0 & 0 & 0 \\
\hline AT211 & $7.214 \mathrm{H}$ & 6.49 & 0 & 6.49 \\
\hline AT217 & $0.0323 \mathrm{~s}$ & 0.0328 & 0 & 0.0328 \\
\hline RN218 & $0.035 \mathrm{~S}$ & 0.104 & 0 & 0.104 \\
\hline RN219 & $3.96 \mathrm{~S}$ & 8.03 & 0.0161 & 8.05 \\
\hline RN220 & $55.61 \mathrm{~S}$ & 0.0721 & 0 & 0.0721 \\
\hline RN222 & $3.8235 \mathrm{D}$ & 0.0537 & 0. & 0.0537 \\
\hline FR221 & $4.8 \mathrm{M}$ & 4.42 & 0 & 4.42 \\
\hline FR223 & $21.8 \mathrm{M}$ & 9.69 & 307 & 316 \\
\hline RA222 & $38 \mathrm{~S}$ & 1.29 & 0 & 1.29 \\
\hline RA223 & 11.4340 & 20.3 & 0 & 20.3 \\
\hline RA224 & $3.62 \mathrm{D}$ & 1.41 & 0 & 1.41 \\
\hline RA225 & $14.8 \mathrm{D}$ & 3.42 & 0 & 3.42 \\
\hline PA226 & $1600 Y$ & 0.977 & 0 & 0.977 \\
\hline RA228 & $5.75 Y$ & $8.02 E-07$ & 0 & $8.02 E-07$ \\
\hline AC225 & $10 \mathrm{D}$ & 3.03 & 0 & 3.03 \\
\hline AC227 & $21.773 Y$ & 0.0862 & 0 & 0.0862 \\
\hline AC228 & $6.13 \mathrm{H}$ & 124 & 379 & 503 \\
\hline TH226 & $30.9 \mathrm{M}$ & 1.5 & 0 & 1.5 \\
\hline TH227 & $18.718 \mathrm{D}$ & 17.1 & 0 & 17.1 \\
\hline TH228 & $1.9132 Y$ & 0.8 & 0 & 0.8 \\
\hline TH229 & $7340 \mathrm{Y}$ & 17 & 0 & 17 \\
\hline TH230 & $77,000 \mathrm{Y}$ & 0.516 & 0 & 0.516 \\
\hline
\end{tabular}

B. 18 
Table B.3. (contd)

\begin{tabular}{|c|c|c|c|c|}
\hline Isotope & Half-Life & Photon & Electron & Total \\
\hline TH231 & $25.52 \mathrm{H}$ & 6.42 & 0 & 6.42 \\
\hline TH232 & $1.4 E+10 Y$ & 0.485 & 0 & 0.485 \\
\hline TH233 & $22.3 \mathrm{M}$ & 5.37 & 428 & 433 \\
\hline TH234 & $24.1 \mathrm{D}$ & 1.77 & 0 & 1.77 \\
\hline PA230 & $17.4 \mathrm{D}$ & 92.5 & 2.56 & 95.1 \\
\hline PA231 & $32,760 Y$ & 6.94 & 0 & 6.94 \\
\hline PA233 & $27 \mathrm{D}$ & 32.8 & 0 & 32.8 \\
\hline PA234 & $6.7 \mathrm{H}$ & 268 & 136 & 404 \\
\hline PA234m & $1.17 \mathrm{M}$ & 1.55 & 1110 & 1110 \\
\hline U230 & $20.8 \mathrm{D}$ & 0.872 & 0 & 0.872 \\
\hline U231 & $4.2 \mathrm{D}$ & 16.2 & 0 & 16.2 \\
\hline U232 & $72 Y$ & 0.742 & 0 & 0.742 \\
\hline U233 & $1.59 E+05 Y$ & 0.261 & 0 & 0.261 \\
\hline U234 & 2.45E+05 $Y$ & 0.636 & 0 & 0.636 \\
\hline U235 & $7.04 E+08 Y$ & 22.7 & 0 & 22.7 \\
\hline U236 & 2.34E+07 $Y$ & 0.599 & 0 & 0.599 \\
\hline U237 & $6.75 \mathrm{D}$ & 24.2 & 0 & 24.2 \\
\hline U238 & 4.47E+09 $\gamma$ & 0.53 & 0 & 0.53 \\
\hline U239 & $23.4 \mathrm{M}$ & 8.04 & 423 & 431 \\
\hline U240 & $14.1 \mathrm{H}$ & 2.83 & 0.195 & 3.02 \\
\hline NP235 & $396.1 \mathrm{D}$ & 2.57 & 0 & 2.57 \\
\hline NP236 & $1.15 E+05 Y$ & 26.8 & 0 & 26.8 \\
\hline NP236m & $22.5 \mathrm{H}$ & 8.98 & 3.64 & 12.6 \\
\hline NP237 & 2.14E+06 Y & 7.54 & 0 & 7.54 \\
\hline NP238 & $2.117 \mathrm{D}$ & 74.7 & 211 & 285 \\
\hline NP239 & $2.355 \mathrm{D}$ & 27.2 & 2.63 & 29.8 \\
\hline NP240 & $65 M$ & 162 & 117 & 279 \\
\hline NP240m & $7.4 \mathrm{M}$ & 46.4 & 770 & 816 \\
\hline PU236 & $2.851 Y$ & 0.811 & 0 & 0.811 \\
\hline PU237 & 45.3 D & 10.1 & 0 & 10.1 \\
\hline PU238 & $87.75 Y$ & 0.717 & 0 & 0.717 \\
\hline PU239 & $24,131 Y$ & 0.28 & 0 & 0.28 \\
\hline PU240 & $6537 Y$ & 0.683 & 0 & 0.683 \\
\hline PU241 & $14.4 Y$ & 0 & 0 & 0 \\
\hline PU242 & $3.76 E+05 Y$ & 0.567 & 0 & 0.567 \\
\hline PU243 & $4.956 \mathrm{H}$ & 4.24 & 9.56 & 13.8 \\
\hline PU244 & 8.26E +07 $Y$ & 0.491 & 0 & 0.491 \\
\hline PU245 & $10.57 \mathrm{H}$ & 58.3 & 166 & 225 \\
\hline PU246 & $10.85 \mathrm{D}$ & 14.6 & 0 & 14.6 \\
\hline AM241 & $432.2 Y$ & 6.39 & 0 & 6.39 \\
\hline$A M 242$ & $16.02 \mathrm{H}$ & 3.96 & 26.2 & 30.1 \\
\hline AM242m & $152 Y$ & 1.97 & 0 & 1.97 \\
\hline AM243 & $7380 Y$ & 10.6 & 0 & 10.6 \\
\hline AM244 & $10.1 \mathrm{H}$ & 116 & 66.2 & 183 \\
\hline AM245 & $122.4 M$ & 5.03 & 144 & 149 \\
\hline AM246 & $25 M$ & 130 & 476 & 606 \\
\hline CM242 & $163.2 \mathrm{D}$ & 0.736 & 0 & 0.736 \\
\hline CM243 & $28.5 Y$ & 21.5 & 0 & 21.5 \\
\hline CM244 & $18.11 Y$ & 0.656 & 0 & 0.656 \\
\hline CM245 & $8500 Y$ & 13.9 & 0 & 13.9 \\
\hline CM246 & $4750 Y$ & 0.584 & 0 & 0.584 \\
\hline CM247 & 1.56E +07 Y & 44.4 & 0.0276 & 44.5 \\
\hline CM248 & $3.39 E+05 Y$ & 0.463 & 0 & 0.463 \\
\hline
\end{tabular}

B. 19 
Table B.3. (contd)

\begin{tabular}{|c|c|c|c|c|}
\hline Isotope & Half-Life & Photon & Electron & Total \\
\hline СМ249 & $64.15 \mathrm{M}$ & 2.62 & 167 & 170 \\
\hline CM250 & $6900 \mathrm{Y}$ & 0 & 0 & 0 \\
\hline BK249 & $320 \mathrm{D}$ & 0 & 0 & 0 \\
\hline BK250 & $3.222 \mathrm{H}$ & 118 & 177 & 295 \\
\hline BK251 & $57 M$ & 0 & 347 & 347 \\
\hline CF248 & $333.5 \mathrm{D}$ & 0.505 & 0 & 0.505 \\
\hline CF249 & $350.6 \mathrm{Y}$ & 47.6 & 0 & 47.6 \\
\hline CF250 & $13.08 Y$ & 0.501 & 0 & 0.501 \\
\hline CF251 & $900 Y$ & 19.9 & 0 & 19.9 \\
\hline CF252 & $2.639 \mathrm{Y}$ & 0.466 & 0 & 0.466 \\
\hline CF253 & $17.81 \mathrm{D}$ & 0.00854 & 0 & 0.00854 \\
\hline CF254 & $60.5 \mathrm{D}$ & $3.95 E-06$ & 0 & $3.95 E-\infty 6$ \\
\hline ES253 & $20.467 \mathrm{D}$ & 0.33 & 0 & 0.33 \\
\hline ES254 & $275.7 \mathrm{D}$ & 6.68 & 0 & 6.68 \\
\hline ES254m & $39.3 \mathrm{H}$ & 79.4 & 71.2 & 151 \\
\hline ES255 & $39.8 \mathrm{D}$ & 0.0361 & 0 & 0.0361 \\
\hline FM254 & $3.24 \mathrm{H}$ & 0.493 & 0 & 0.493 \\
\hline FM255 & $20.07 \mathrm{H}$ & 4.04 & 0 & 4.04 \\
\hline FM256 & $157.6 \mathrm{M}$ & 0 & 0 & 0 \\
\hline
\end{tabular}

Spaces were inserted between the element name and 1 \& 2 digit mass numbers so they would be the same as ORIGEN2 nuclide names.

The name of AG106m was changed to AG106 so it would be the same as the ORIGEN2 name.

The names NB 92 and NB 92m were interchanged so nuclide NB 92 would be consistent with the ORIGEN2 name. 



\section{Appendix C}

\section{External Dose-Rate Conversion Factors}




\section{Appendix C}

\section{External Dose-Rate Conversion Factors}

Dose-rate conversion factors for external exposure to photons and electrons emitted by radionuclides deposited on the ground surface are given by Kocher and Eckerman. ${ }^{\text {(a) }}$ Dose-rate factors are given for a number of internal organs as well as the skin. Effective dose-rate factors were calculated by Kocher and Eckerman by using weighing factors for specific internal body organs. The larger of the effective dose-rate factor and the total skin dose-rate factor for each radionuclide was multiplied by the Hanford total radionuclide activity in curies to give a radiation hazard value.

Comparison amongst nuclides gives a relative radiation hazard.

(a) Kocher, D. C., and K. F. Eckerman. 1988. External Dose-Rate Conversion Factors for Calculation of Dose to the Public; DOE/EH-0070, U.S. Department of Energy, Washington, D.C., and 1989 Erratum Sheet.

$$
\text { C. } 1
$$




\section{Distribution}

No. of

Copies

Offsite

2 DOE Office of Scientific and Technical Information

W. Fuhr

AFTAC

1030 S. Hwy A1A

Patrick, AFB, FL 32925-6001

\section{Onsite}

Westinghouse Hanford Company

D. J. Brown

D. J. Hoff

Bechtel Hainford Inc.

S. Peterson
No. of

Copies

DOE Richland Operations Office

R. B. Goranson

9 Pacific Northwest Laboratory

R. W. Perkins

U. P. Jenquin

N. A. Wogman

Publishing Coordination

Technical Report Files (5) 